\title{
Stereodivergent complexity-to-diversity strategy en route to the synthesis of nature-inspired skeleta
}

Vunnam Srinivasulu, ${ }^{\dagger}$ Gourishetty Srikanth, ${ }^{\ddagger}$ Monther A. Khanfar, ${ }^{\ddagger}$ Imad A. Abu-Yousef, ${ }^{\ddagger}$ Amin F. Majdalawieh, ${ }^{\ddagger}$ Ralph Mazitschek, " Subbaiah Chennam Setty, ${ }^{\ddagger}$ Anusha Sebastian, ${ }^{\dagger}$ and Taleb H. Al$\mathrm{Tel}^{\dagger \wedge *}$

${ }^{\dagger}$ Sharjah Institute for Medical Research, University of Sharjah, P.O. Box 27272, Sharjah, UAE

$¥$ Department of Biology, Chemistry and Environmental Sciences, American University of Sharjah, P.O. Box 26666, Sharjah, UAE

${ }^{£}$ College of Science, Department of Chemistry, Pure and applied chemistry group, University of Sharjah, P.O. Box 27272, Sharjah, UAE

"Center for Systems Biology, Massachusetts General Hospital, Boston, MA.

${ }^{\wedge}$ College of Pharmacy, University of Sharjah, P.O. Box 27272, Sharjah, UAE

*Corresponding author: taltal@sharjah.ac.ae

List of Contents

X-ray Diffraction Data

Schemes

Spectra

References
S1

S2-S11

S11-S12

S13-S99

S102 
X-Ray diffraction studies: The crystals were obtained as follows. The corresponding compound was dissolved methanol, ethyl acetate mixture by boiling. The resulting solution was allowed to cool down to room temperature undisturbed, which resulted in the formation of fine crystals.

Data collection, reduction, and cell refinement were performed using the software package CrysAlisPro1. Analytical absorption corrections were applied using spherical harmonics implemented in SCALE3 (ABSPACK) scaling algorithm. Using Olex22 program, the structures were solved with SHELXL-973 and/or ShelXT4 structure solution programs, using direct method or intrinsic phasing and refined with Olex2 refine5 refinement package using GaussNewton or Least Squares minimization. Anisotropic least-squares refinement of non-H atoms was applied. All crystallographic plots were obtained using the Olex2 program. A summary of the crystallographic data and structure refinement parameters for all the compounds is given below in the Tables (Table 1 to 4). Crystallographic data (excluding structure factors) for the structures in this paper have been deposited with the Cambridge Crystallographic Data Centre, CCDC, 12 Union Road, Cambridge CB21EZ, UK. Copies of the data can be obtained free of charge on quoting the depository number CCDC 2075391 for (20a), CCDC 2093065 (33a) and CCDC 2093067 (35e). (Fax: +44-1223-336-033; E-Mail: deposit@ccdc.cam.ac.uk, http:// www.ccdc.cam.ac.uk). 


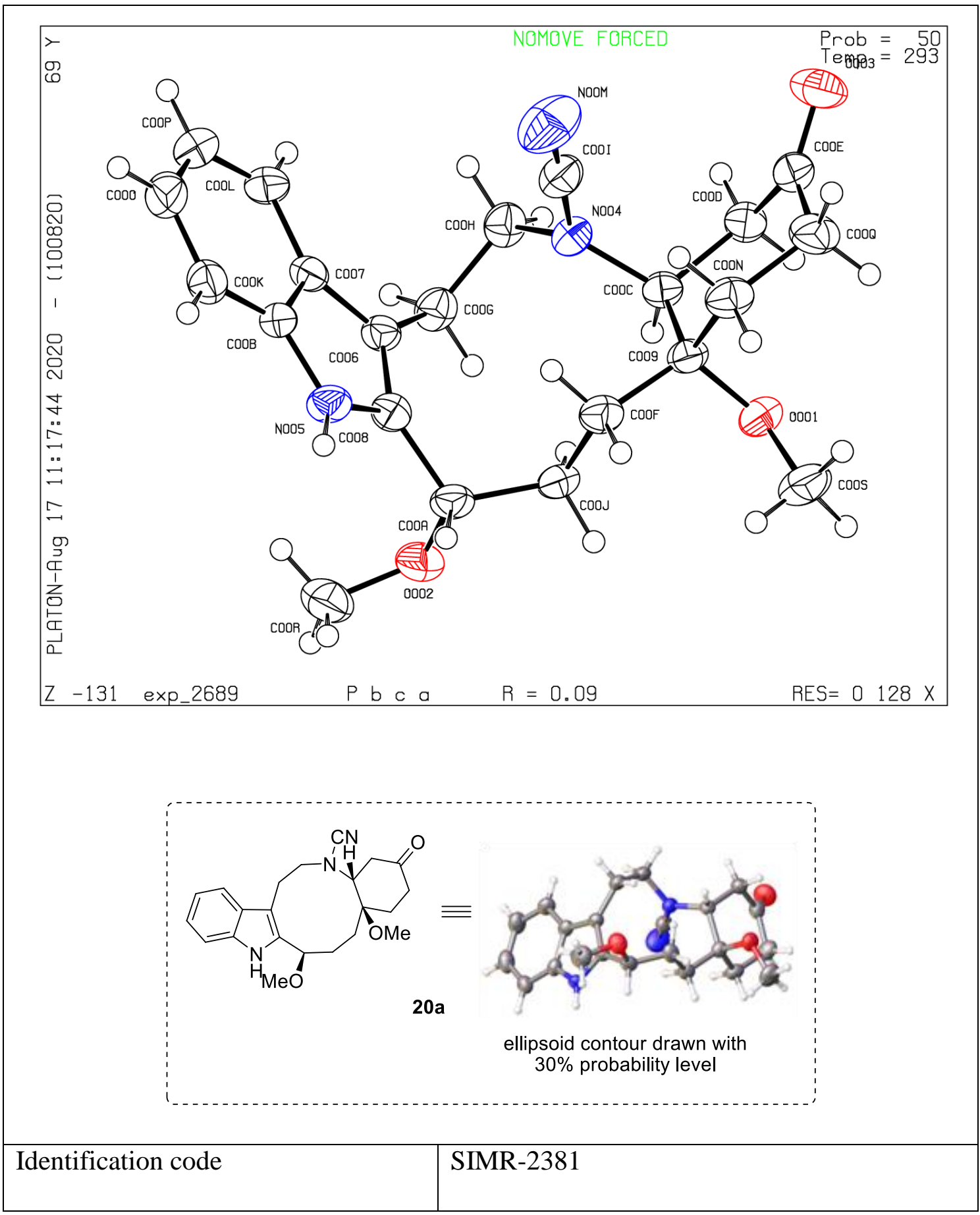




\begin{tabular}{|l|l|}
\hline & Exp_2689 \\
\hline Empirical formula & $\mathrm{C}_{22} \mathrm{H}_{27} \mathrm{~N}_{3} \mathrm{O}_{3}$ \\
\hline Formula weight & 381.46 \\
\hline Temperature & $293(2) \mathrm{K}$ \\
\hline Wavelength & $0.71073 \AA$ \\
\hline Crystal system & orthorhombic \\
\hline Space group & Pbca \\
\hline Unit cell dimensions & $\mathrm{a}=12.374(2) \AA$ \\
& $\mathrm{b}=14.868(3) \AA$ \\
& $\mathrm{c}=21.630(3) \AA$ \\
\hline Volume & $\AA=90^{\circ}$ \\
\hline Z & $3979.4(11) \AA^{\circ}$ \\
\hline Density (calculated) & 8 \\
\hline Absorption coefficient & $1.273 \mathrm{~g} / \mathrm{cm}^{3}$ \\
\hline F(000) & $0.086 \mathrm{~mm}^{\circ}$ \\
\hline Crystal size & 1632.0 \\
\hline 2 Theta range for data collection & 5.704 to $61.816^{\circ}$. \\
\hline Index ranges & $-11 \leq \mathrm{h} \leq 17,-19 \leq \mathrm{k} \leq 17,-31 \leq 1 \leq 30$ \\
\hline Reflections collected & 15462 \\
\hline Independent reflections & $5664\left[\mathrm{R}_{\text {int }}=0.0874\right]$ \\
\hline Completeness to theta $=25.242^{\circ}$ & $99.8 \%$ \\
\hline
\end{tabular}




\begin{tabular}{|l|l|}
\hline Max. and min. transmission & 1.000 and 0.874 \\
\hline Refinement method & SHELXL \\
\hline Data / restraints / parameters & $5664 / 0 / 255$ \\
\hline Goodness-of-fit on F2 & 1.033 \\
\hline Final R indices [I>2sigma(I)] & $\mathrm{R}_{1}=0.0936, \mathrm{wR}_{2}=0.1151$ \\
\hline R indices (all data) & $\mathrm{R}_{1}=0.2213, \mathrm{wR}_{2}=0.1505$ \\
\hline Largest diff. peak and hole & 0.20 and $-0.19 \mathrm{e} . \AA^{-3}$ \\
\hline
\end{tabular}




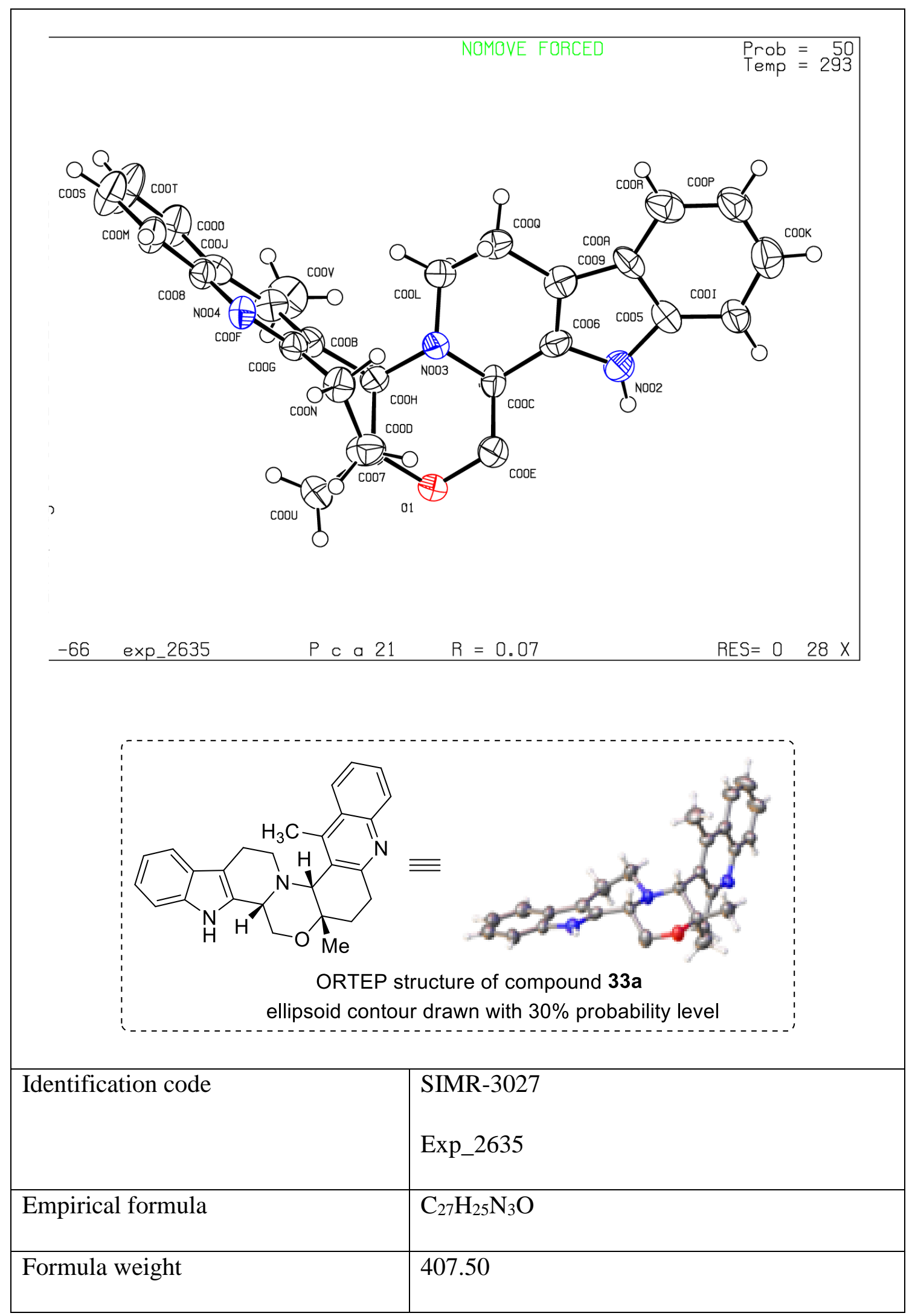




\begin{tabular}{|c|c|}
\hline Temperature & 293(2) K \\
\hline Wavelength & $0.71073 \AA$ \\
\hline Crystal system & orthorhombic \\
\hline Space group & Pca2 $2_{1}$ \\
\hline Unit cell dimensions & $\begin{array}{ll}\mathrm{a}=16.6556(19) \AA & \alpha=90^{\circ} \\
\mathrm{b}=12.2553(12) \AA & \beta=90^{\circ} \\
\mathrm{c}=13.2599(15) \AA & \gamma=90^{\circ}\end{array}$ \\
\hline Volume & $2706.6(5) \AA^{3}$ \\
\hline $\mathrm{Z}$ & 4 \\
\hline Density (calculated) & $1.000 \mathrm{~g} / \mathrm{cm}^{3}$ \\
\hline Absorption coefficient & $0.062 \mathrm{~mm}^{-1}$ \\
\hline $\mathrm{F}(000)$ & 864.0 \\
\hline Crystal size & $0.18 \times 0.08 \times 0.04 \mathrm{~mm}^{3}$ \\
\hline 2 Theta range for data collection & 6.146 to $58.78^{\circ}$. \\
\hline Index ranges & $-22 \leq \mathrm{h} \leq 22, \quad-10 \leq \mathrm{k} \leq 16, \quad-18 \leq 1 \leq 16$ \\
\hline Reflections collected & 12524 \\
\hline Independent reflections & $5814\left[\mathrm{R}_{\text {int }}=0.0609\right]$ \\
\hline Completeness to theta $=25.242^{\circ}$ & $99.7 \%$ \\
\hline Absorption correction & multi-scan \\
\hline Max. and min. transmission & 0.284 and 1.000 \\
\hline Refinement method & SHELXL \\
\hline Data / restraints / parameters & $5814 / 1 / 282$ \\
\hline
\end{tabular}




\begin{tabular}{|l|l|}
\hline Goodness-of-fit on F2 & 1.013 \\
\hline Final R indices [I>2sigma(I)] & $\mathrm{R}_{1}=0.0723, \mathrm{wR}_{2}=0.1581$ \\
\hline R indices (all data) & $\mathrm{R}_{1}=0.1174, \mathrm{wR}_{2}=0.1835$ \\
\hline Largest diff. peak and hole & 0.22 and $-0.21 \mathrm{e} . \AA^{-3}$ \\
\hline
\end{tabular}




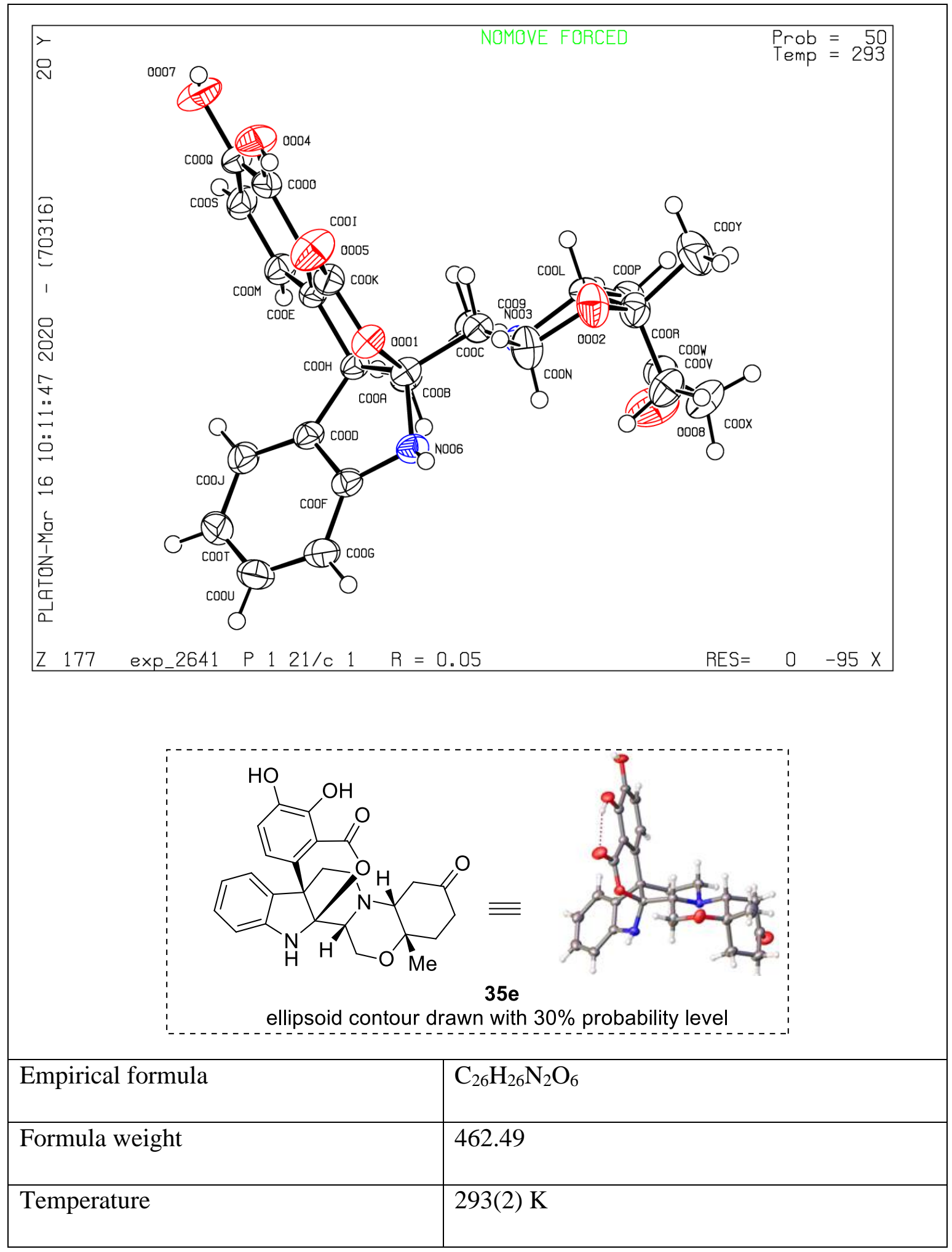




\begin{tabular}{|l|l|}
\hline Wavelength & $0.71073 \AA$ \\
\hline Crystal system & monoclinic \\
\hline Space group & $\mathrm{P} 2{ }_{1} / \mathrm{c}$ \\
\hline Unit cell dimensions & $\mathrm{a}=13.5211(8) \AA \quad \alpha=90^{\circ}$ \\
& $\mathrm{b}=7.7949(4) \AA \quad \quad \quad \beta=90.373(6)^{\circ}$ \\
& $\mathrm{c}=20.7246(12) \AA \quad \gamma=90^{\circ}$ \\
\hline Volume & $2184.2(2) \AA^{3}$ \\
\hline Z & 4 \\
\hline Density (calculated) & $1.406 \mathrm{~g} / \mathrm{cm}^{3}$ \\
\hline Absorption coefficient & $0.101 \mathrm{~mm}^{-1}$ \\
\hline F(000) & 976.0 \\
\hline Crystal size & $0.18 \times 0.08 \times 0.04 \mathrm{~mm}^{3}$ \\
\hline 2 Theta range for data collection & 6.026 to $59.058^{\circ}$. \\
\hline Index ranges & $-18 \leq \mathrm{h} \leq 14,-10 \leq \mathrm{k} \leq 10,-27 \leq 1 \leq 28$ \\
\hline Reflections collected & 18277 \\
\hline Independent reflections & $5340\left[\mathrm{R}_{\text {int }}=0.0464\right]$ \\
\hline Completeness to theta $=25.242^{\circ}$ & $99.8 \%$ \\
\hline Absorption correction & multi-scan \\
\hline Max. and min. transmission & $0.802 \mathrm{and} 1.000$ \\
\hline Refinement method & $\mathrm{SHELXL}$ \\
\hline Data / restraints / parameters & $5340 / 0 / 310$ \\
\hline Goodness-of-fit on F2 & 1.065 \\
\hline
\end{tabular}




\begin{tabular}{|l|l|}
\hline Final $\mathrm{R}$ indices [I>2sigma(I)] & $\mathrm{R}_{1}=0.0503, \mathrm{wR}_{2}=0.1300$ \\
\hline $\mathrm{R}$ indices (all data) & $\mathrm{R}_{1}=0.0779, \mathrm{wR}_{2}=0.1493$ \\
\hline Largest diff. peak and hole & 0.26 and $-0.39 \mathrm{e} . \AA^{-3}$ \\
\hline
\end{tabular}

Scheme 1. General Scheme S1: for the Preparation of 3a-e and 4a-e ${ }^{[1]}$

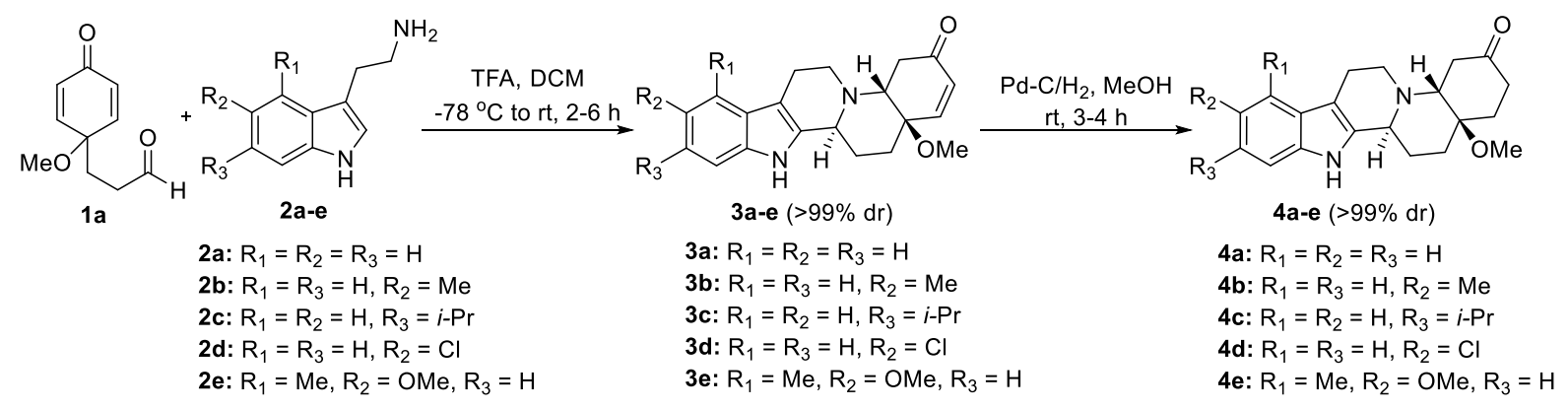

Scheme S2. Preparation of compounds 3f-g, 7 and 32 ${ }^{[1]}$<smiles>COC1(CO)C=CC(=O)C=C1</smiles>

$1 \mathrm{~b}$<smiles>NCCc1c[nH]c2ccccc12</smiles>

2a
TFA, DCM $-78^{\circ} \mathrm{C}$ to rt, $2-6 \mathrm{~h}$<smiles>CO[C@]12C=CC(=O)C[C@H]1N1CCc3c([nH]c4ccccc34)C1CO2</smiles>

$3 f$<smiles>CO[C@]12C=CC(=O)C[C@H]1N1CCc3c([nH]c4ccccc34)[C@H]2CO1</smiles>

$\mathrm{Pd}-\mathrm{C} / \mathrm{H}_{2}, \mathrm{MeOH}$ $\mathrm{rt}, 3 \mathrm{~h}$<smiles>CO[C@]12CCC(=O)C[C@H]1N1CCc3c([nH]c4ccccc34)[C@H]2CO1</smiles>

$\mathrm{Pd}-\mathrm{C} / \mathrm{H}_{2}, \mathrm{MeOH}$ $\mathrm{rt}, 3 \mathrm{~h}$

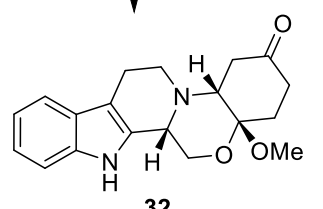

Scheme S3. Preparation of compounds $3 \mathrm{~h}$ and $10 \mathrm{a}^{[1]}$<smiles>COC1(CCC=O)C=CC(=O)C=C1</smiles>

$1 \mathrm{a}$

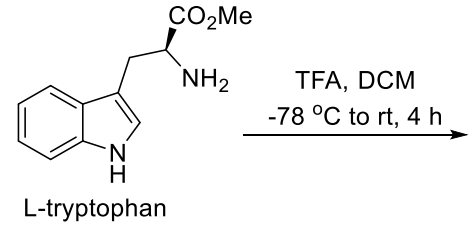

L-tryptophan<smiles>COC12C=CC(=O)CC1(OC)N1CCC2c2[nH]c3ccccc3c2CC1C(C)=O</smiles>

3h (>99\% ee)
$\mathrm{Pd}-\mathrm{C} / \mathrm{H}_{2}, \mathrm{MeOH}$

$\mathrm{rt}, 3 \mathrm{~h}$<smiles>COC(=O)C1Cc2c([nH]c3ccccc23)C2CCN1[C@@]1(OC)CC(=O)CC[C@]21OC</smiles>

10a (>99\% ee) 
Scheme S4. General Scheme for the Preparation of $3 i-j, 10 b$ and $13^{[1]}$

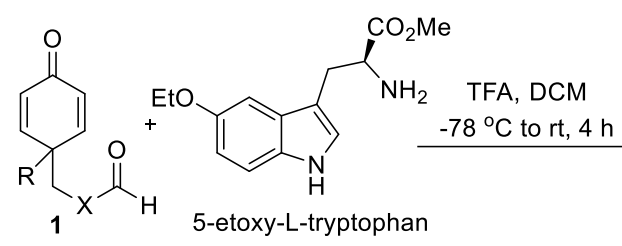

1a: $\mathrm{X}=\mathrm{CH}_{2}, \mathrm{R}=\mathrm{OMe}$

1b: $X=O, R=M e$

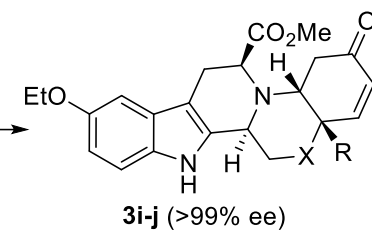

3i: $\mathrm{X}=\mathrm{CH}_{2}, \mathrm{R}=\mathrm{OMe}$

3j: $X=O, R=M e$

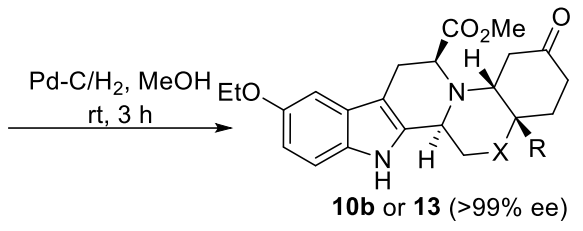

10b: $X=\mathrm{CH}_{2}, \mathrm{R}=\mathrm{OMe}$ 13: $X=O, R=M e$ 


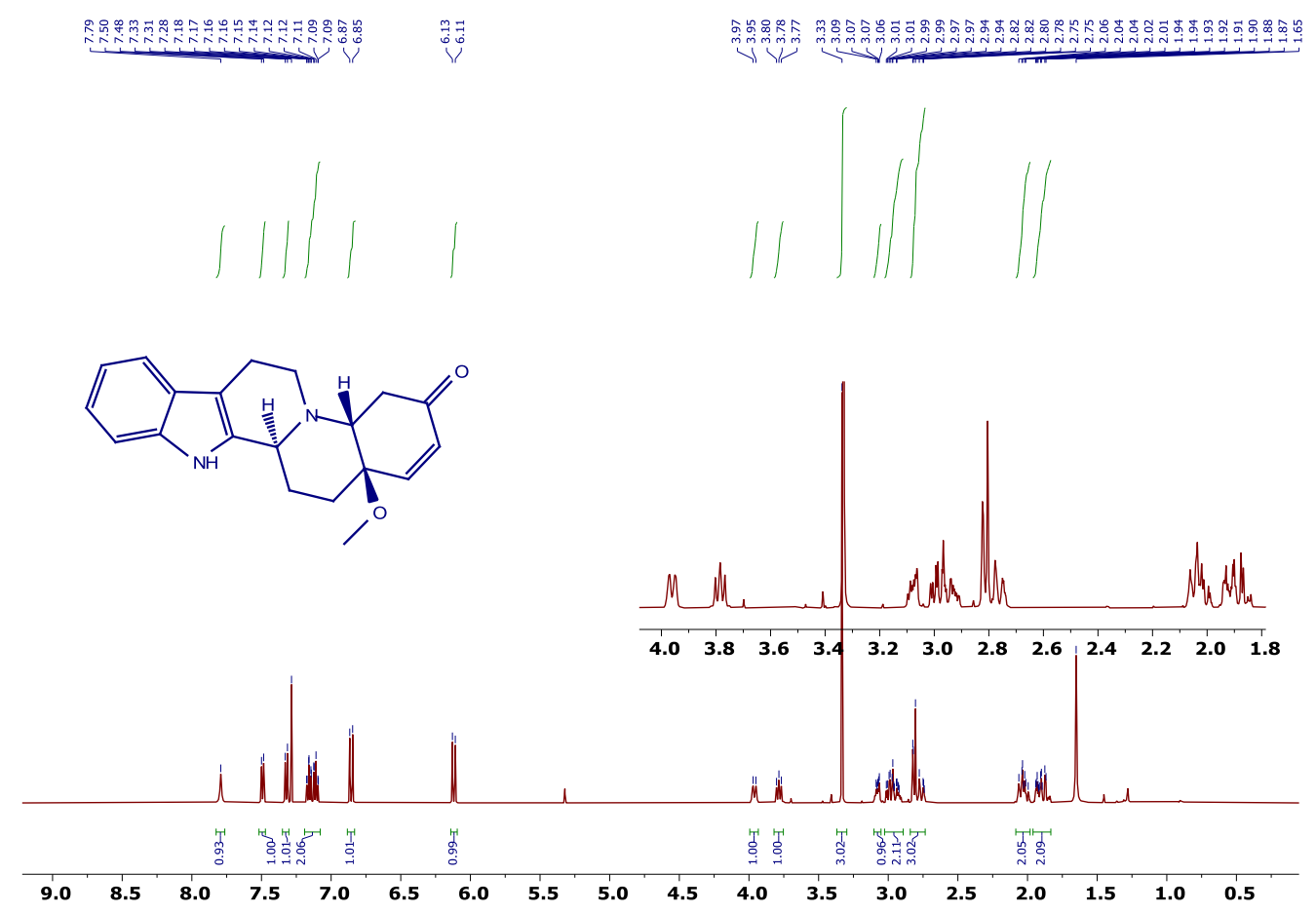

Figure S1 ${ }^{1} \mathrm{H}$ NMR $\left(500 \mathrm{MHz}, \mathrm{CDCl}_{3}\right.$ ) spectrum of (2aR,6aR,14bS)-2a-methoxy-2,2a,6,6a, 8,9,14,14boctahydroindolo[2',3':3,4]pyrido[1,2-a]quinolin-5(1H)-one (3a)
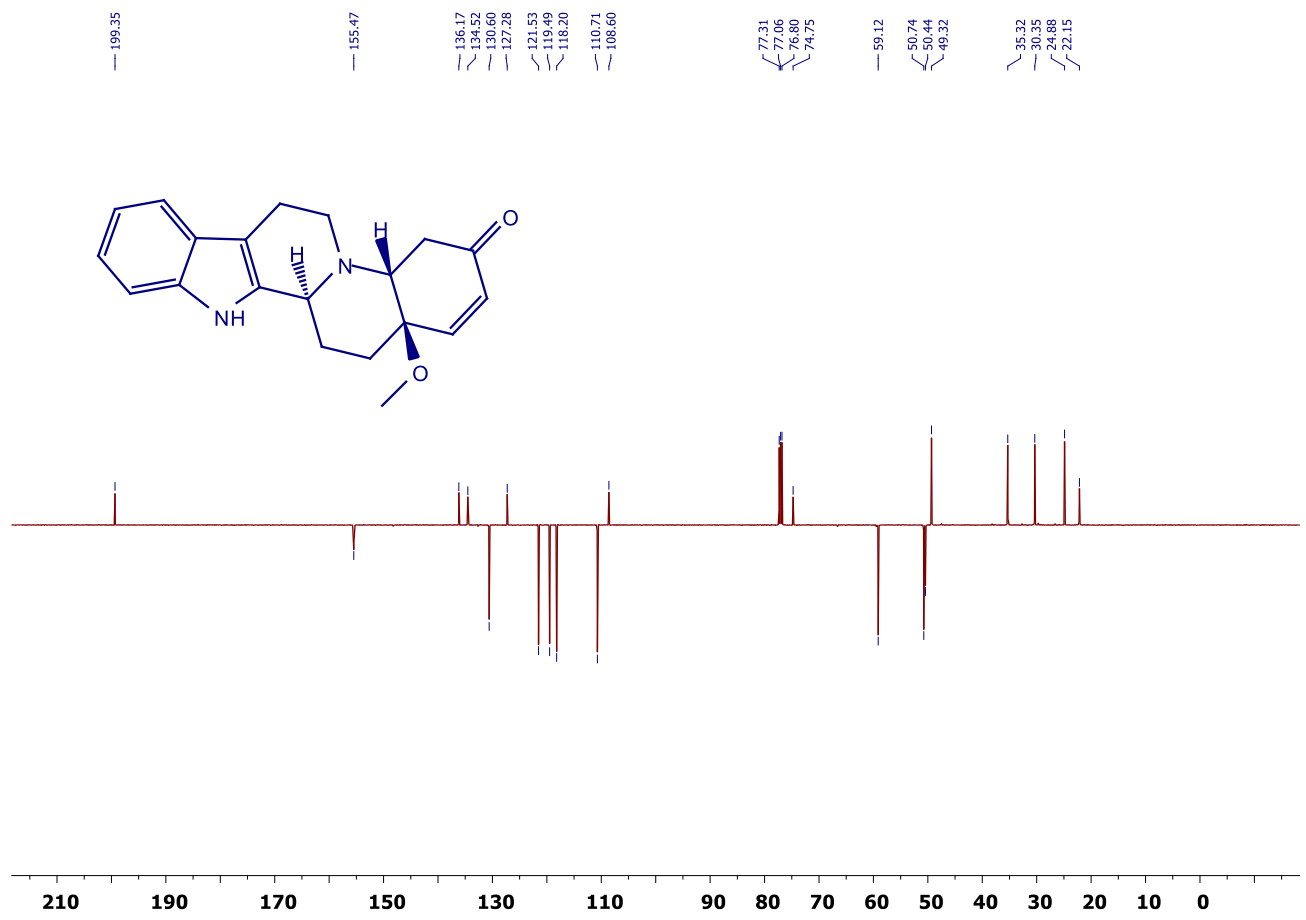

Figure S2 ${ }^{13} \mathrm{C}\left\{{ }^{1} \mathrm{H}\right\}$ APT NMR (125 MHz, $\left.\mathrm{CDCl}_{3}\right)$ spectrum of (2aR,6aR,14bS)-2a-methoxy2,2a,6,6a,8,9,14,14b-octahydroindolo[2',3':3,4]pyrido[1,2-a]quinolin-5(1H)-one (3a) 
<smiles>CCC(=O)CN1[C@@]2(OC)CC[C@@]3(OC)c4[nH]c5ccc(C)cc5c4CC[C@]13C2</smiles>
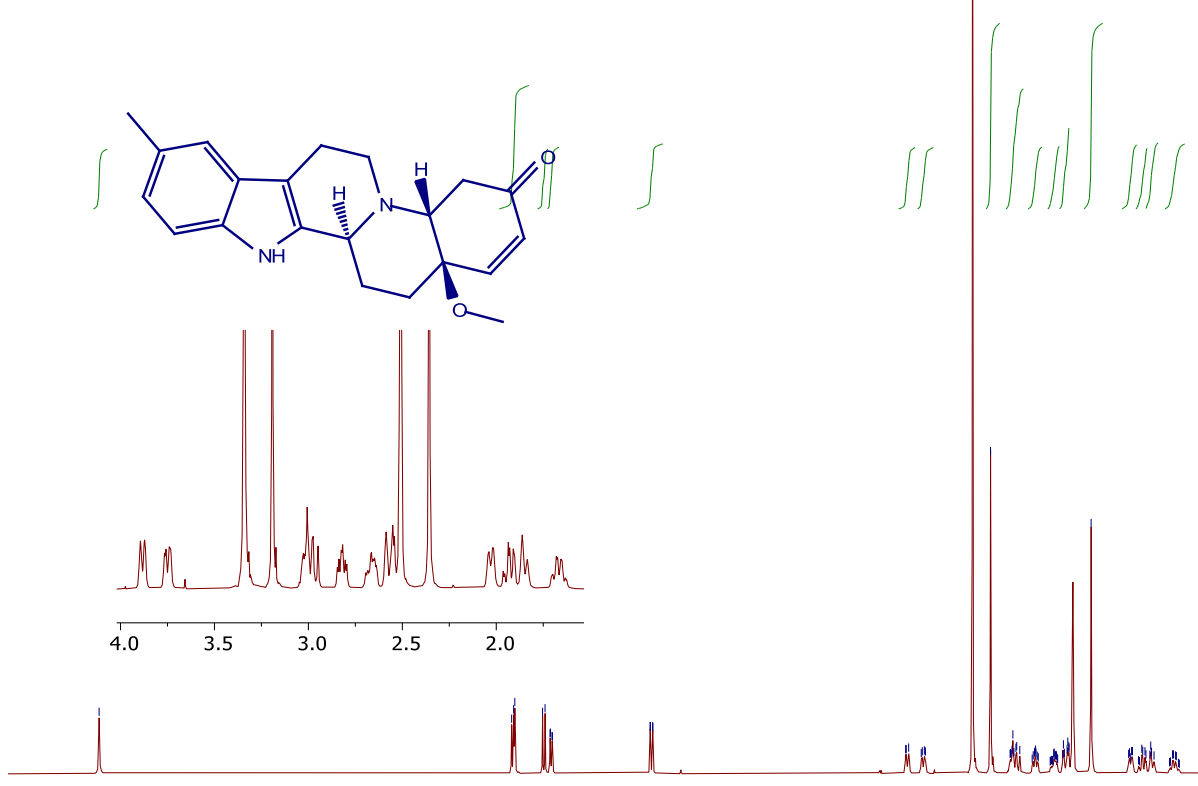

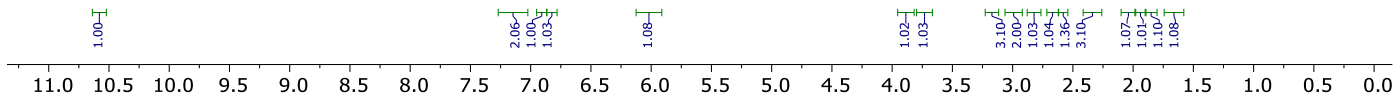

Figure S3 ${ }^{1} \mathrm{H}$ NMR (500 MHz, DMSO-d6) spectrum of (2aR,6aR,14bS)-2a-methoxy-11-methyl2,2a,6,6a,8,9,14,14b-octahydroindolo[2',3':3,4]pyrido[1,2-a]quinolin-5(1H)-one (3b)
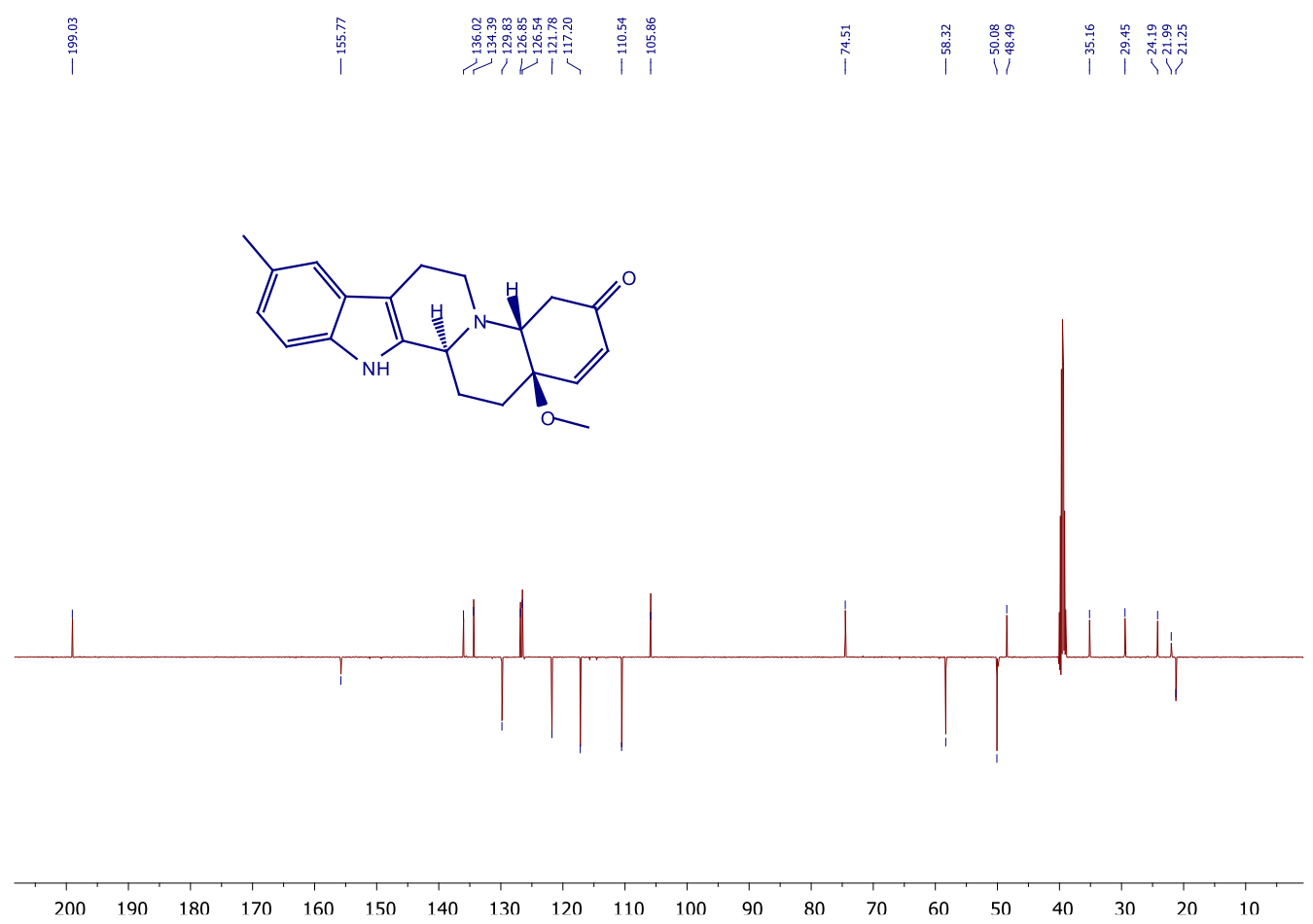

Figure S4 ${ }^{13} \mathrm{C}\left\{{ }^{1} \mathrm{H}\right\}$ APT NMR (125 MHz, DMSO-d6) spectrum of (2aR,6aR,14bS)-2a-methoxy-11methyl-2,2a,6,6a,8,9,14,14b-octahydroindolo[2',3':3,4]pyrido[1,2-a]quinolin-5(1H)-one (3b) 


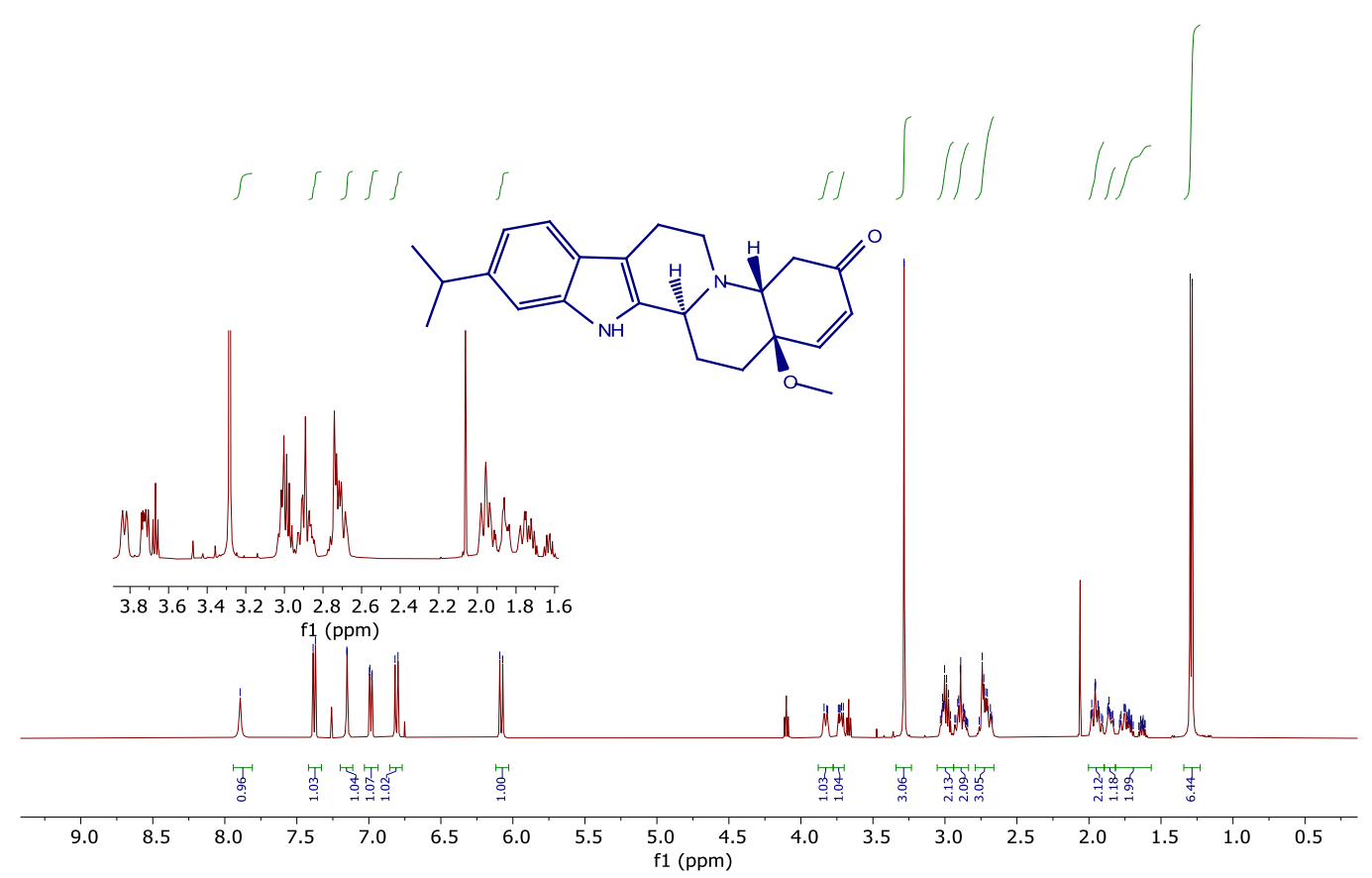

Figure S5 ${ }^{1} \mathrm{H}$ NMR $\left(500 \mathrm{MHz}, \mathrm{CDCl}_{3}\right)$ spectrum of (2aR,6aR,14bS)-12-isopropyl-2a-methoxy2,2a,6,6a, 8,9,14,14b-octahydroindolo[2',3':3,4]pyrido[1,2-a]quinolin-5(1H)-one (3c)
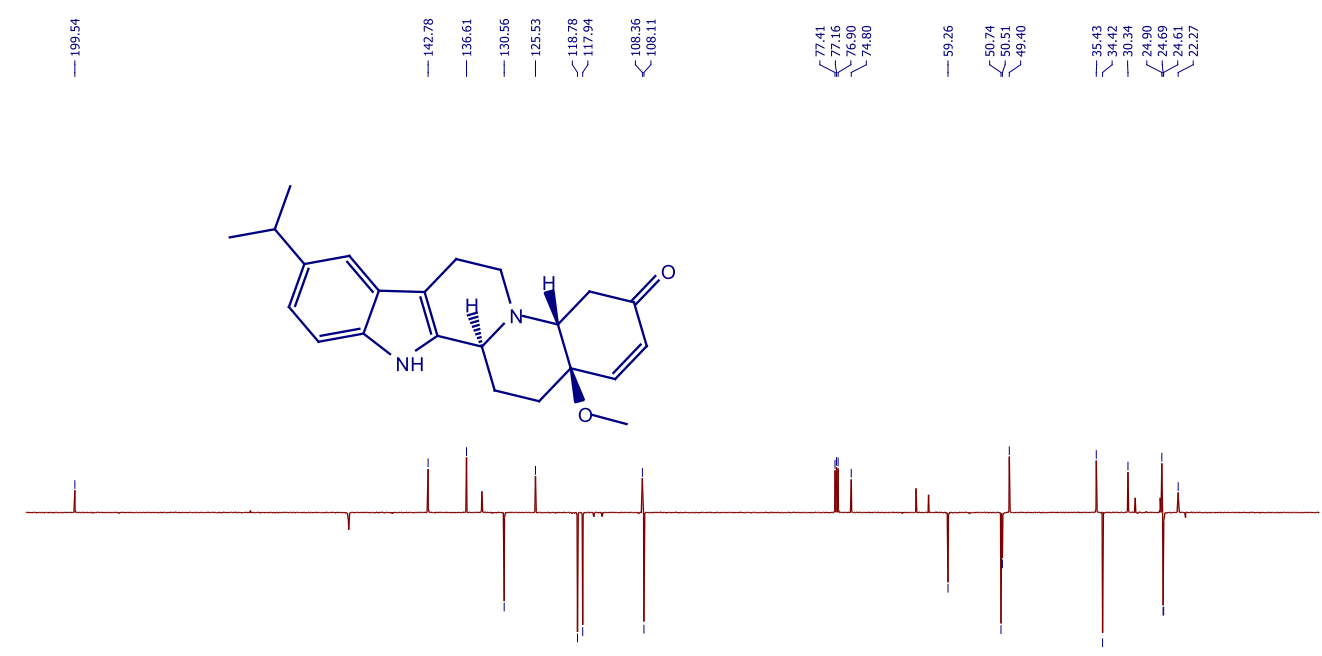

$\begin{array}{lllllllllllllllllllllllllllllll}200 & 190 & 180 & 170 & 160 & 150 & 140 & 130 & 120 & 110 & 100 & 90 & 80 & 70 & 60 & 50 & 40 & 30 & 20 & 10 & 0\end{array}$

Figure S6 ${ }^{13} \mathrm{C}\left\{{ }^{1} \mathrm{H}\right\}$ APT NMR $\left(125 \mathrm{MHz}, \mathrm{CDCl}_{3}\right)$ spectrum of (2aR,6aR,14bS)-12-isopropyl-2amethoxy-2,2a,6,6a,8,9,14,14b-octahydroindolo[2',3':3,4]pyrido[1,2-a]quinolin-5(1H)-one (3c) 


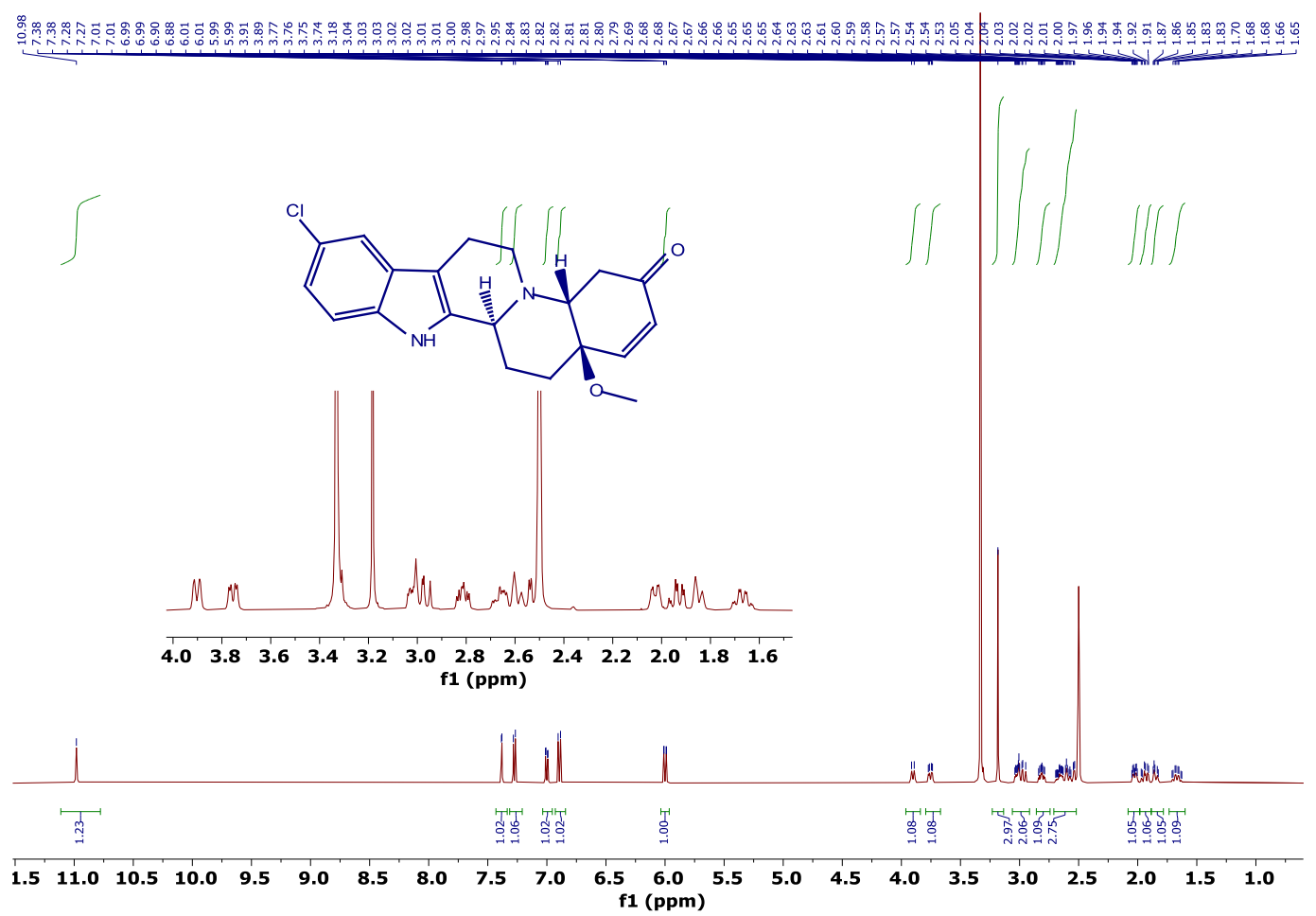

Figure S7 ${ }^{1} \mathrm{H}$ NMR (500 MHz, DMSO-d6) spectrum of (2aR,6aR,14bS)-11-chloro-2a-methoxy2,2a,6,6a, 8,9,14,14b-octahydroindolo[2',3':3,4]pyrido[1,2-a]quinolin-5(1H)-one (3d)
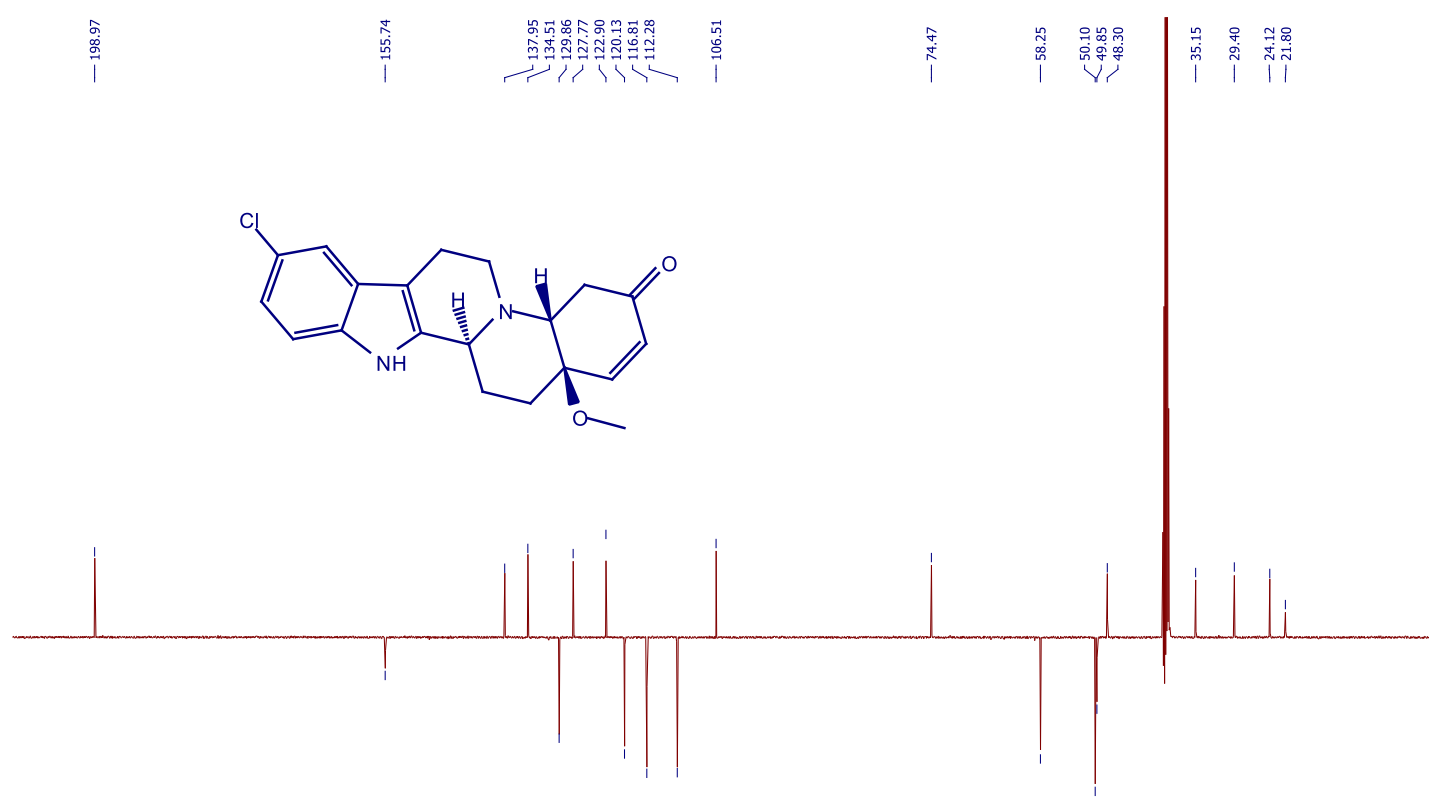

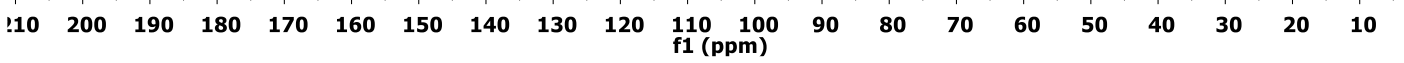

Figure S8 ${ }^{13} \mathrm{C}\left\{{ }^{1} \mathrm{H}\right\}$ APT NMR (125 MHz, DMSO-d6) spectrum of (2aR,6aR,14bS)-11-chloro-2amethoxy-2,2a,6,6a, 8,9,14,14b-octahydroindolo[2',3':3,4]pyrido[1,2-a]quinolin-5(1H)-one (3d) 


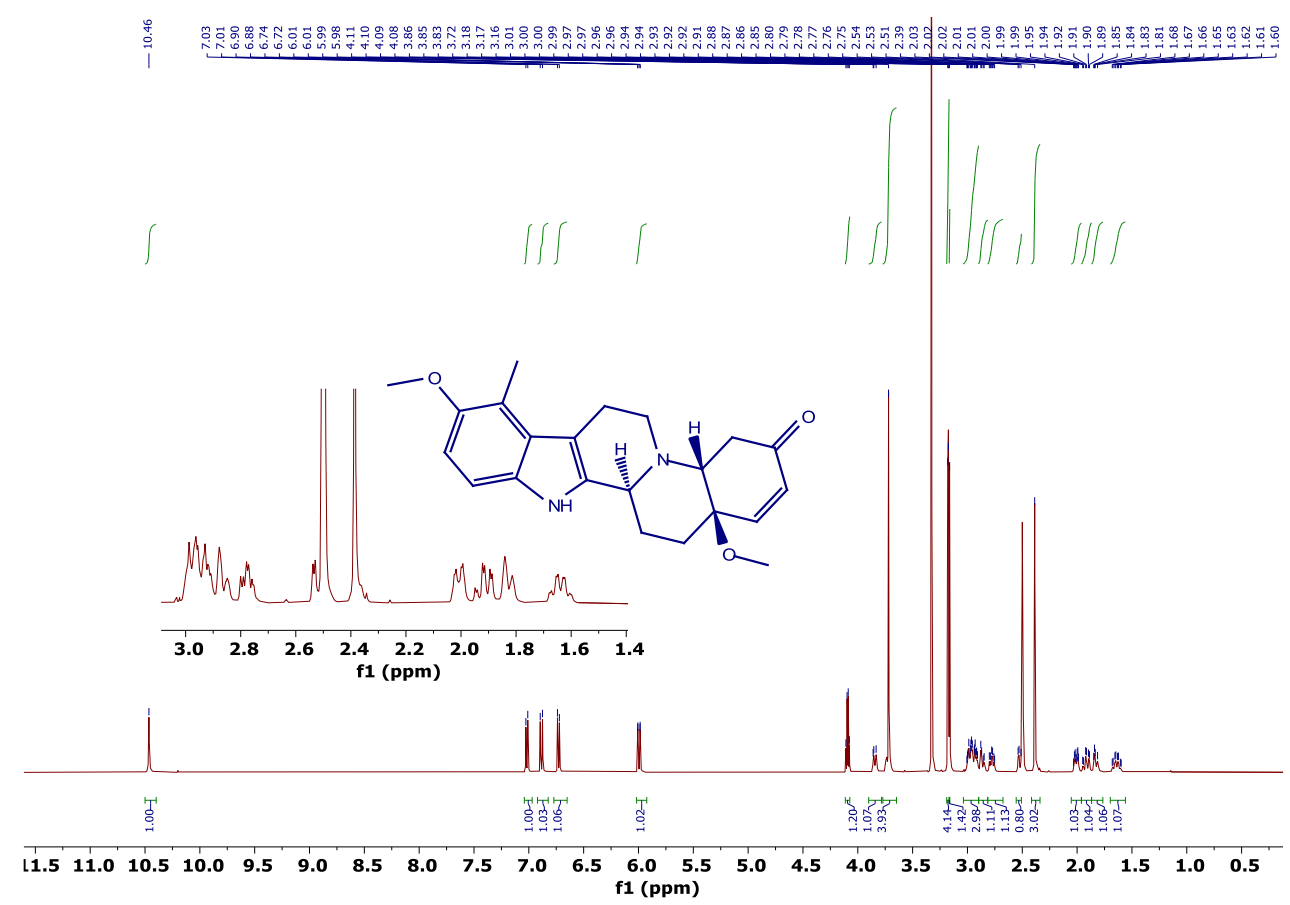

Figure S9 ${ }^{1} \mathrm{H}$ NMR (500 MHz, DMSO-d6) spectrum of (2aR,6aR,14bS)-2a,11-dimethoxy-10-methyl2,2a,6,6a,8,9,14,14b-octahydroindolo[2',3':3,4]pyrido[1,2-a]quinolin-5(1H)-one

(3e)
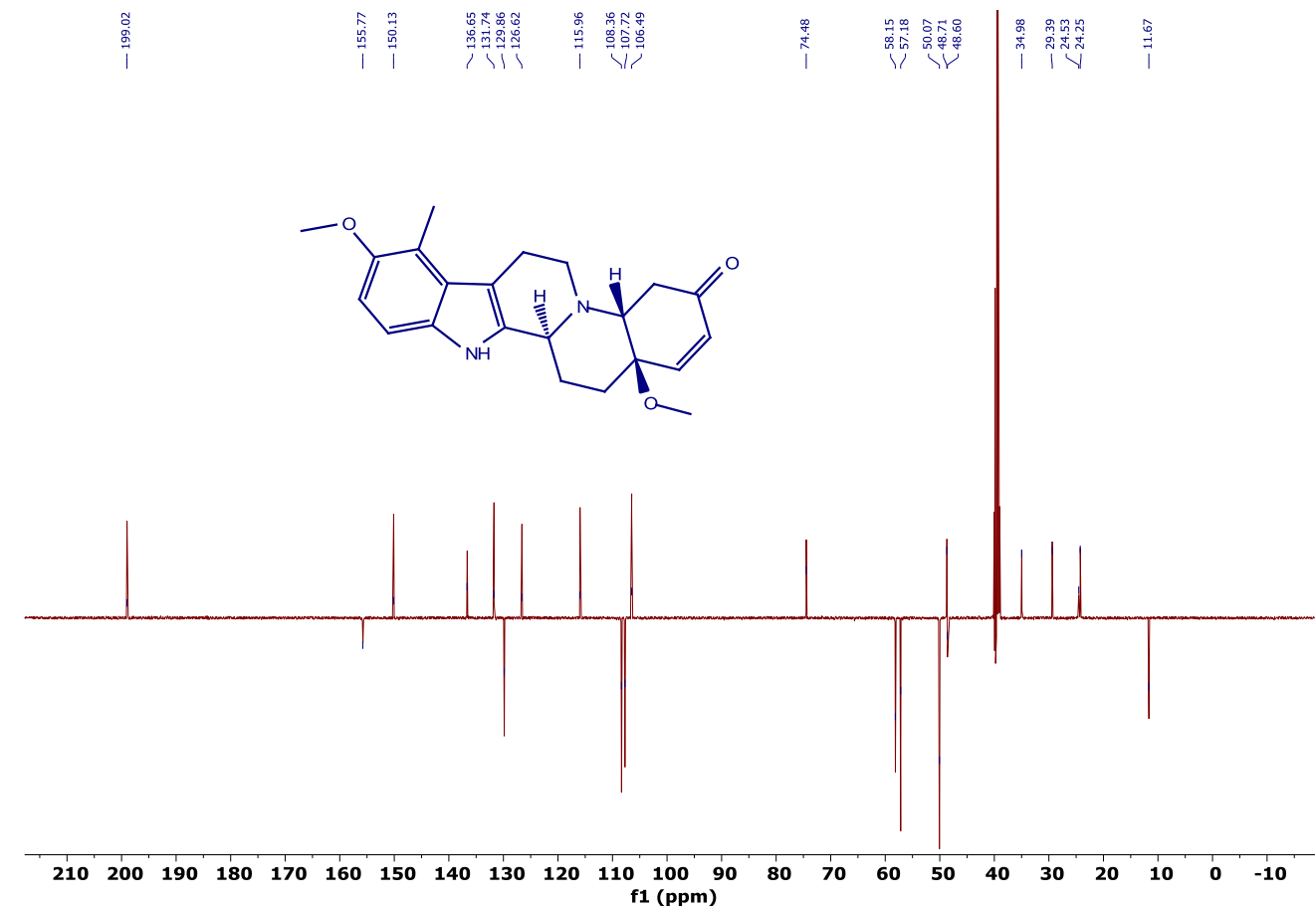

Figure S10 ${ }^{13} \mathrm{C}\left\{{ }^{1} \mathrm{H}\right\}$ APT NMR (125 MHz, DMSO-d6) spectrum of (2aR,6aR,14bS)-2a,11-dimethoxy10-methyl-2,2a,6,6a,8,9,14,14b-octahydroindolo[2',3':3,4]pyrido[1,2-a]quinolin-5(1H)-one 


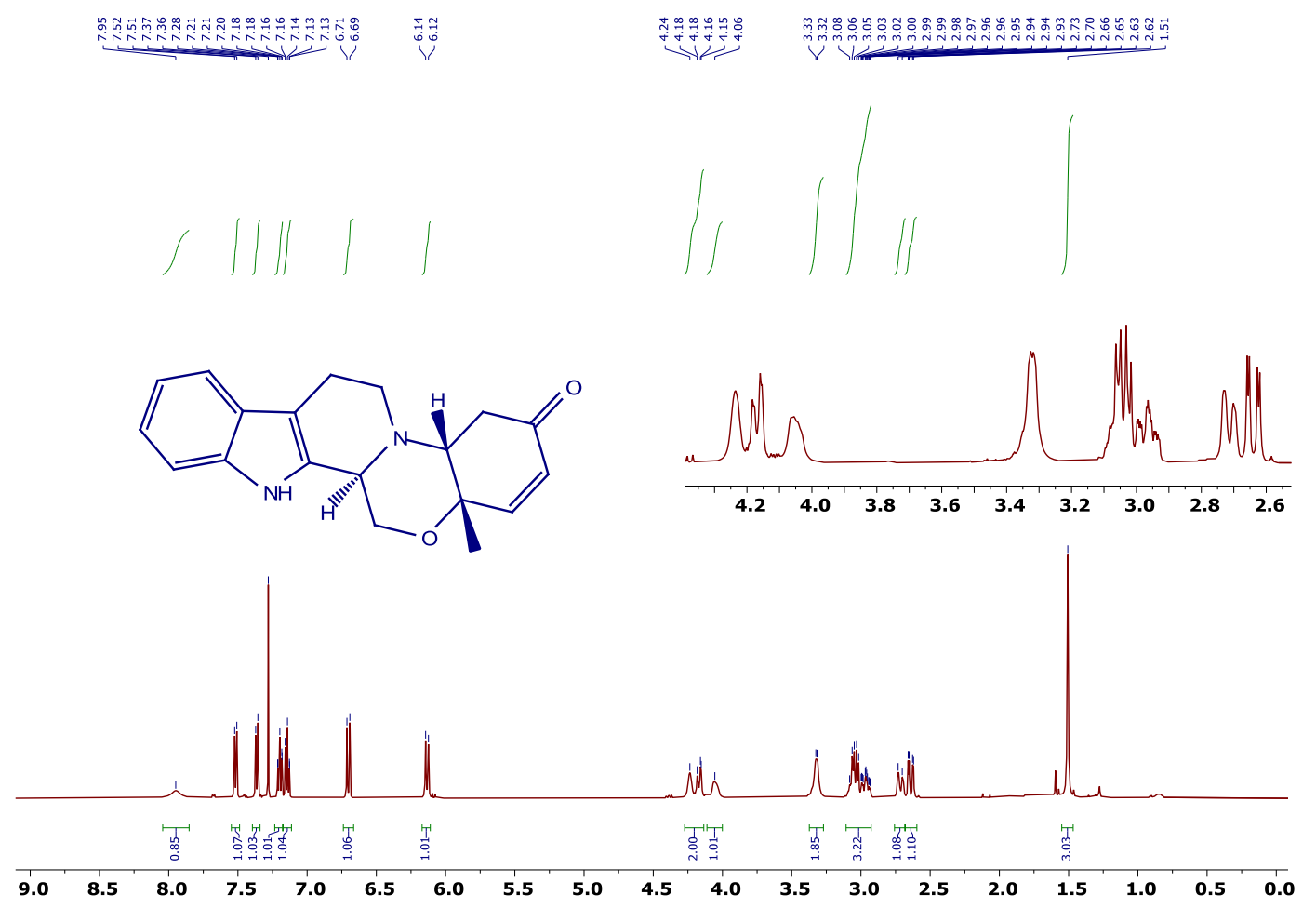

Figure S11 ${ }^{1} \mathrm{H}$ NMR (500 MHz, $\mathrm{CDCl}_{3}$ ) spectrum of (2aS,6aR,14bR)-2a-methyl-6,6a, 8,9,14,14bhexahydro-1H-benzo[5',6'][1,4] oxazino[4',3':1,2]pyrido[3,4-b]indol-5(2aH)-one (3f)

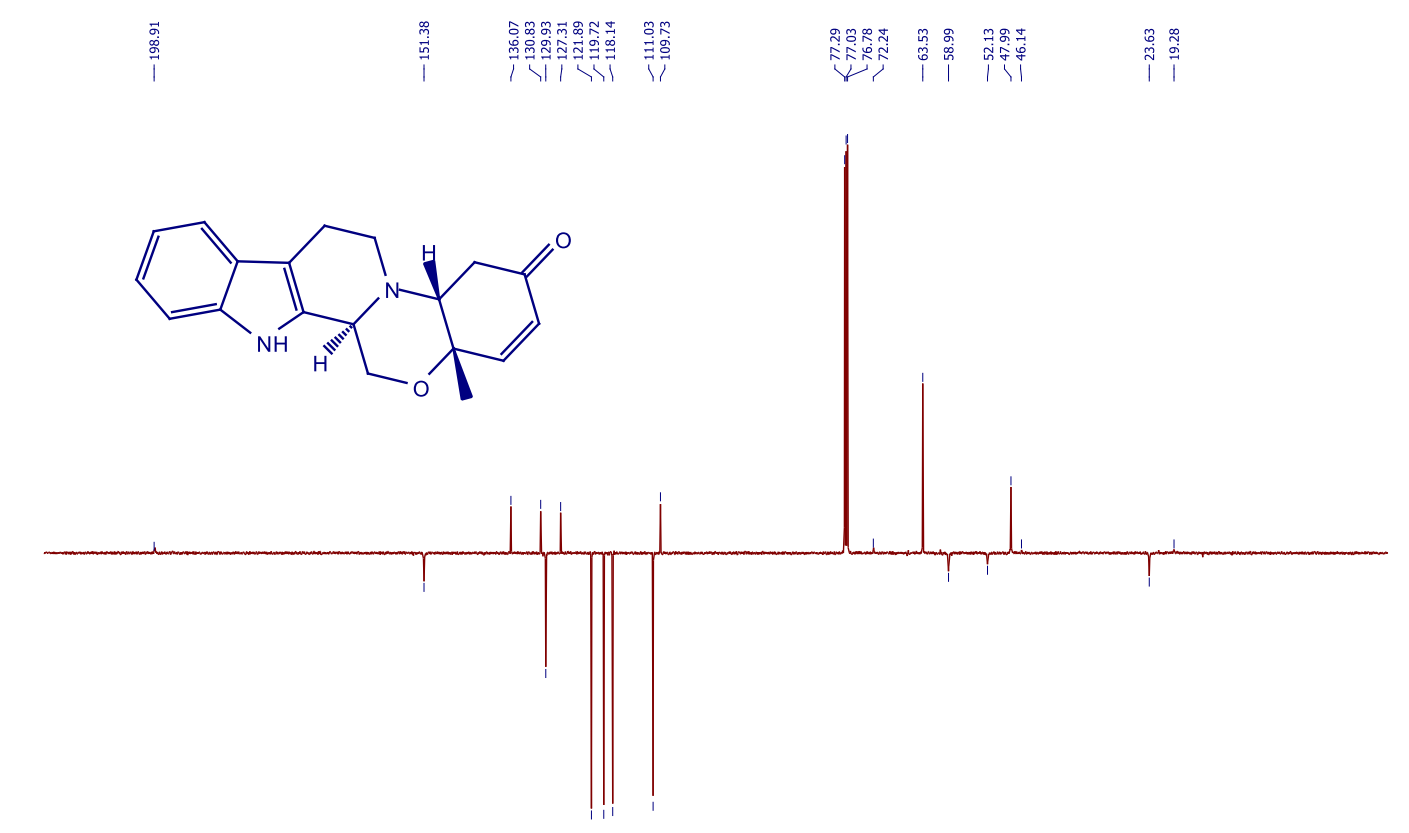

$\begin{array}{llllllllllllllllllll}210 & 190 & 170 & 150 & 130 & 110 & 90 & 80 & 70 & 60 & 50 & 40 & 30 & 20 & 10 & 0 & \end{array}$

Figure S12 ${ }^{13} \mathrm{C}\left\{{ }^{1} \mathrm{H}\right\}$ APT NMR $\left(125 \mathrm{MHz}, \mathrm{CDCl}_{3}\right)$ spectrum of $(2 \mathrm{aS}, 6 \mathrm{aR}, 14 \mathrm{bR})-2 \mathrm{a}-$-methyl6,6a, 8,9,14,14b-hexahydro-1H-benzo[5',6'][1,4] oxazino[4',3':1,2]pyrido[3,4-b]indol-5(2aH)-one (3f) 

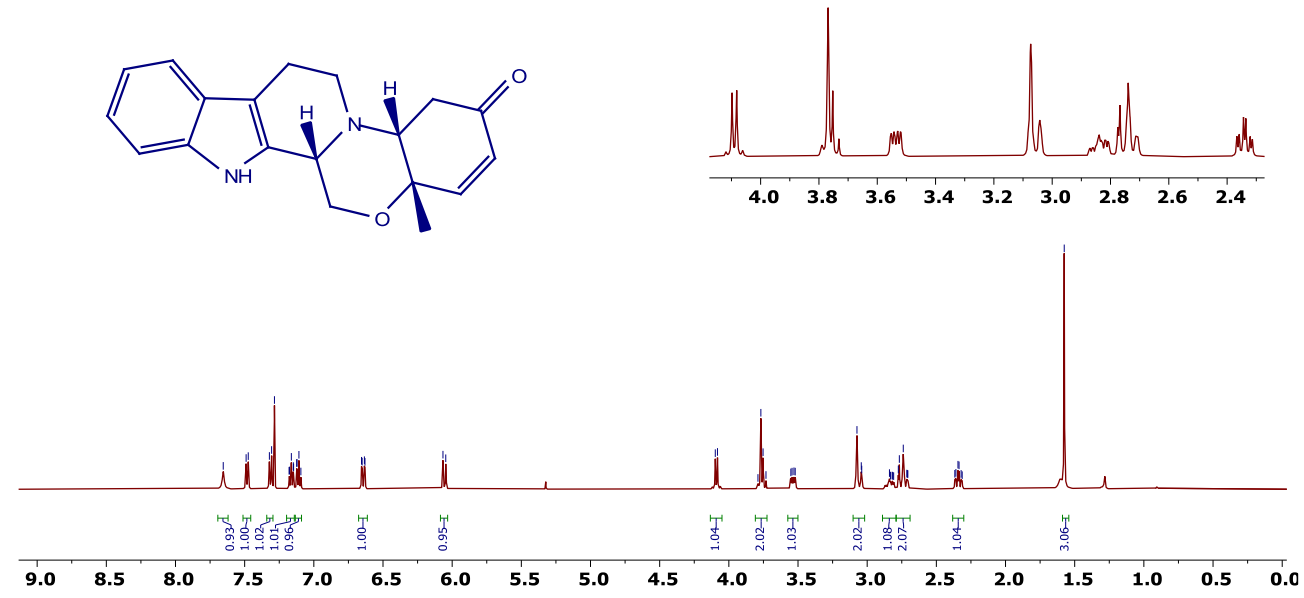

Figure S13 ${ }^{1} \mathrm{H}$ NMR (500 MHz, $\mathrm{CDCl}_{3}$ ) spectrum of (2aS,6aR,14bS)-2a-methyl-6,6a,8,9,14,14bhexahydro-1H-benzo[5',6'][1,4]oxazino[4',3':1,2]pyrido[3,4-b]indol-5(2aH)-one (3g)

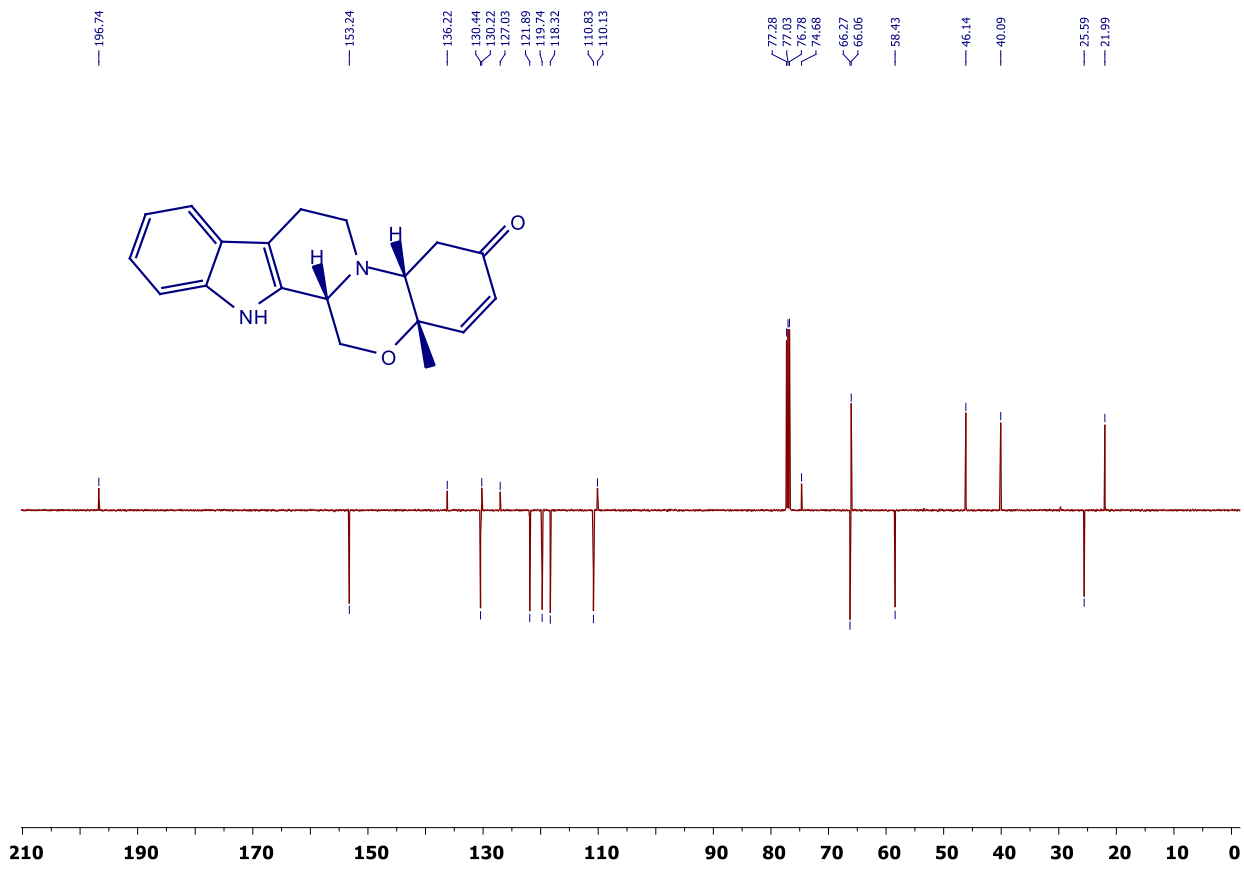

Figure $\mathbf{S 1 4}{ }^{13} \mathrm{C}\left\{{ }^{1} \mathrm{H}\right\}$ APT NMR (125 MHz, CDCl ) spectrum of (2aS,6aR,14bS)-2a-methyl6,6a,8,9,14,14b-hexahydro-1H-benzo[5',6'][1,4]oxazino[4',3':1,2]pyrido[3,4-b]indol-5(2aH)-one (3g) 

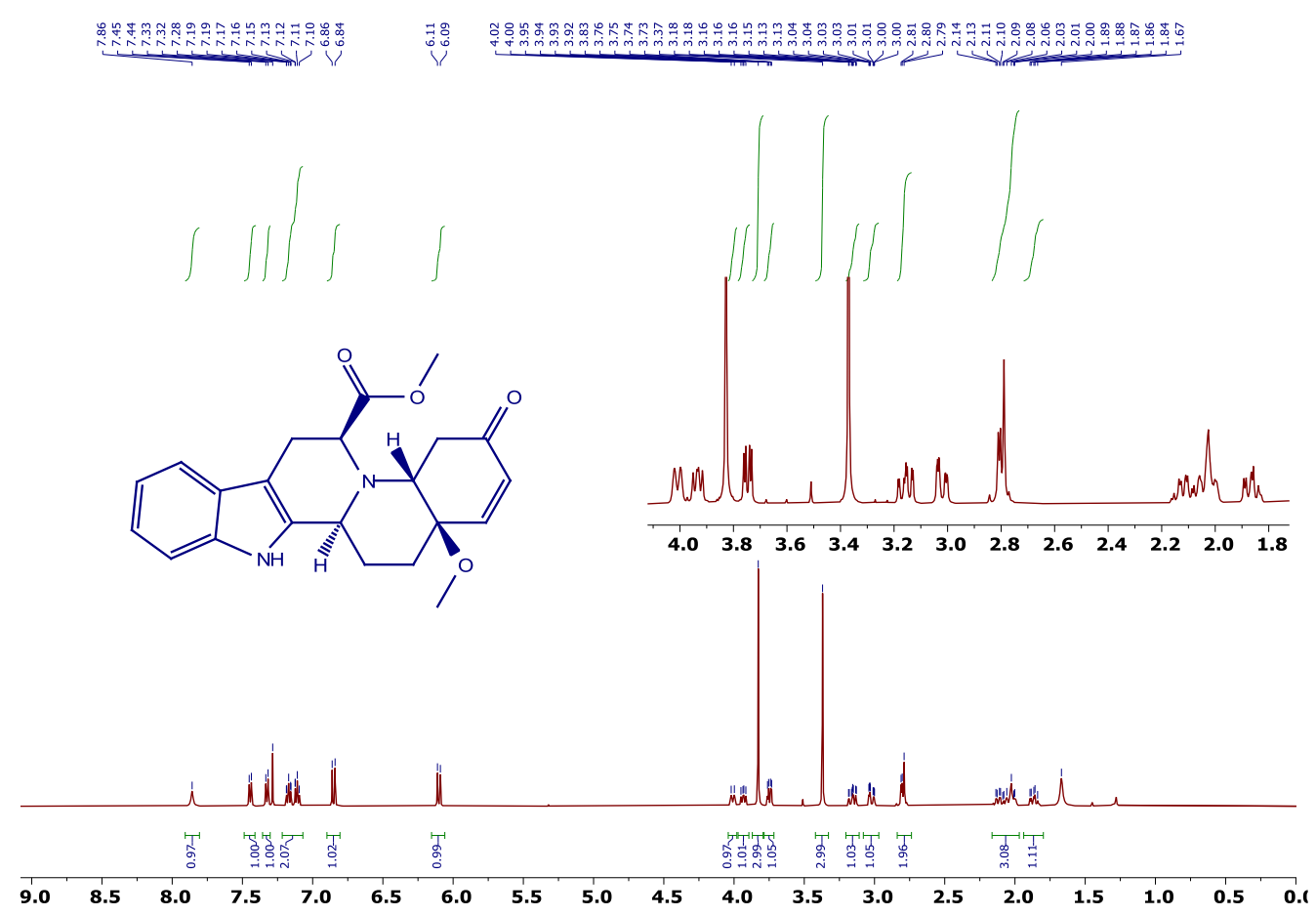

Figure S15 ${ }^{1} \mathrm{H}$ NMR (500 MHz, $\left.\mathrm{CDCl}_{3}\right)$ spectrum of methyl (2aR,6aR,8S,14bS)-2a-methoxy-5-oxo$1,2,2 \mathrm{a}, 5,6,6 \mathrm{a}, 8,9,14,14 \mathrm{~b}$-decahydroindolo[2',3':3,4]pyrido[1,2-a]quinoline-8-carboxylate (3h)

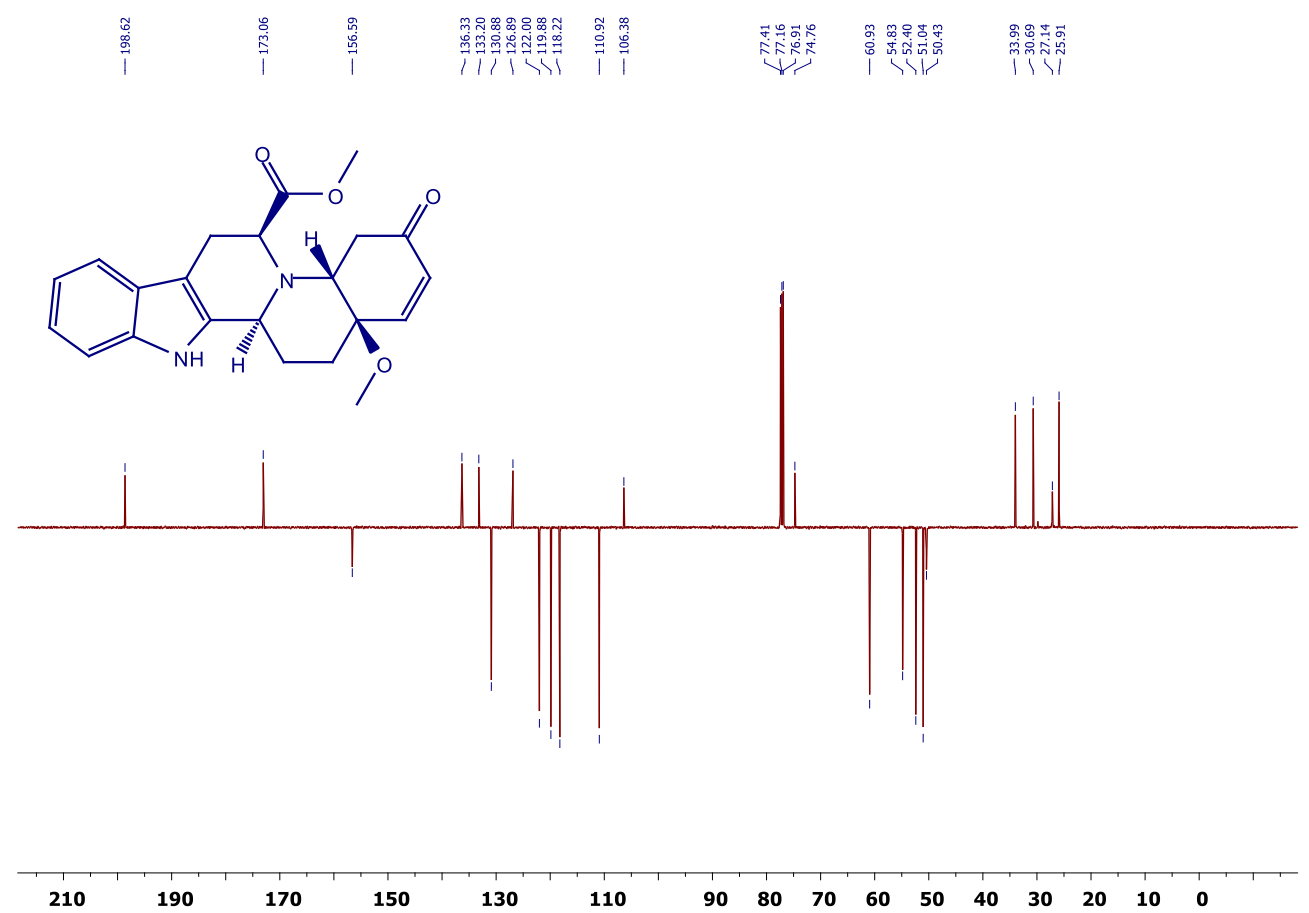

Figure S16 ${ }^{13} \mathrm{C}\left\{{ }^{1} \mathrm{H}\right\}$ APT NMR $\left(125 \mathrm{MHz}, \mathrm{CDCl}_{3}\right)$ spectrum of methyl (2aR,6aR,8S,14bS)-2amethoxy-5-oxo-1,2,2a,5,6,6a,8,9,14,14b-decahydroindolo[2',3':3,4]pyrido[1,2-a]quinoline-8-carboxylate (3h) 


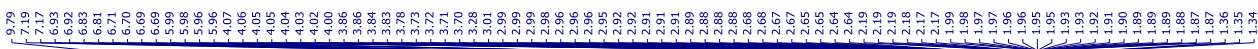

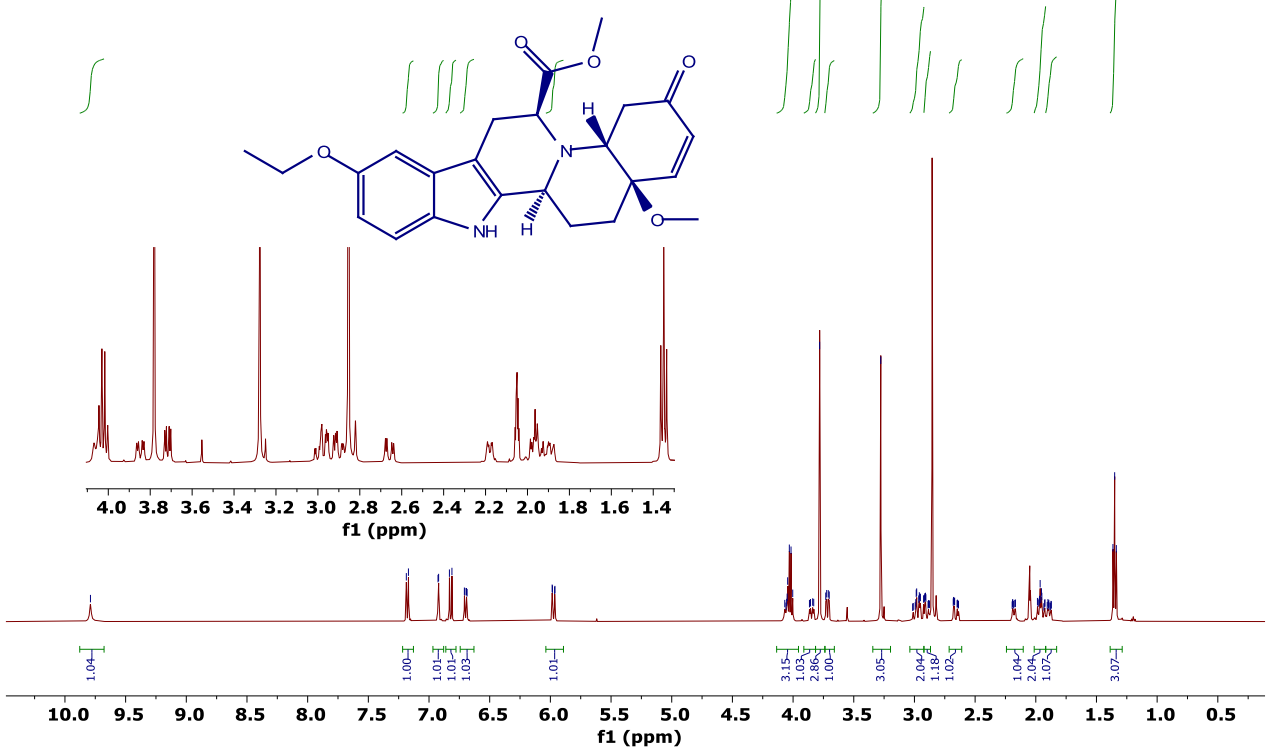

Figure S17 ${ }^{1} \mathrm{H}$ NMR (500 MHz, acetone-d6) spectrum of (2aR,6aR,14bS)-2a,11-dimethoxy-10-methyl2,2a,6,6a,8,9,14,14b-octahydroindolo[2',3':3,4]pyrido[1,2-a]quinolin-5(1H)-one

(3i)
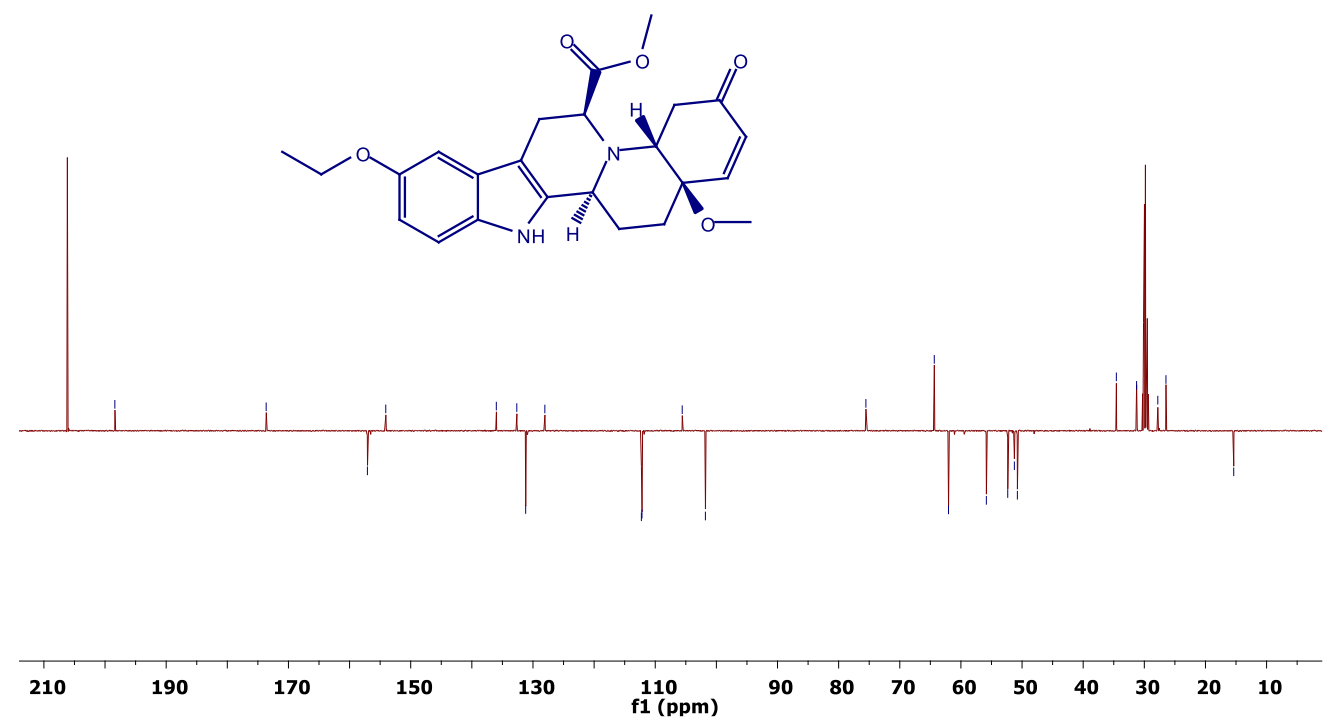

Figure S18 ${ }^{13} \mathrm{C}\left\{{ }^{1} \mathrm{H}\right\}$ APT NMR (125 MHz, acetone-d6) spectrum of (2aR,6aR,14bS)-2a,11-dimethoxy10-methyl-2,2a,6,6a,8,9,14,14b-octahydroindolo[2',3':3,4]pyrido[1,2-a]quinolin-5(1H)-one 


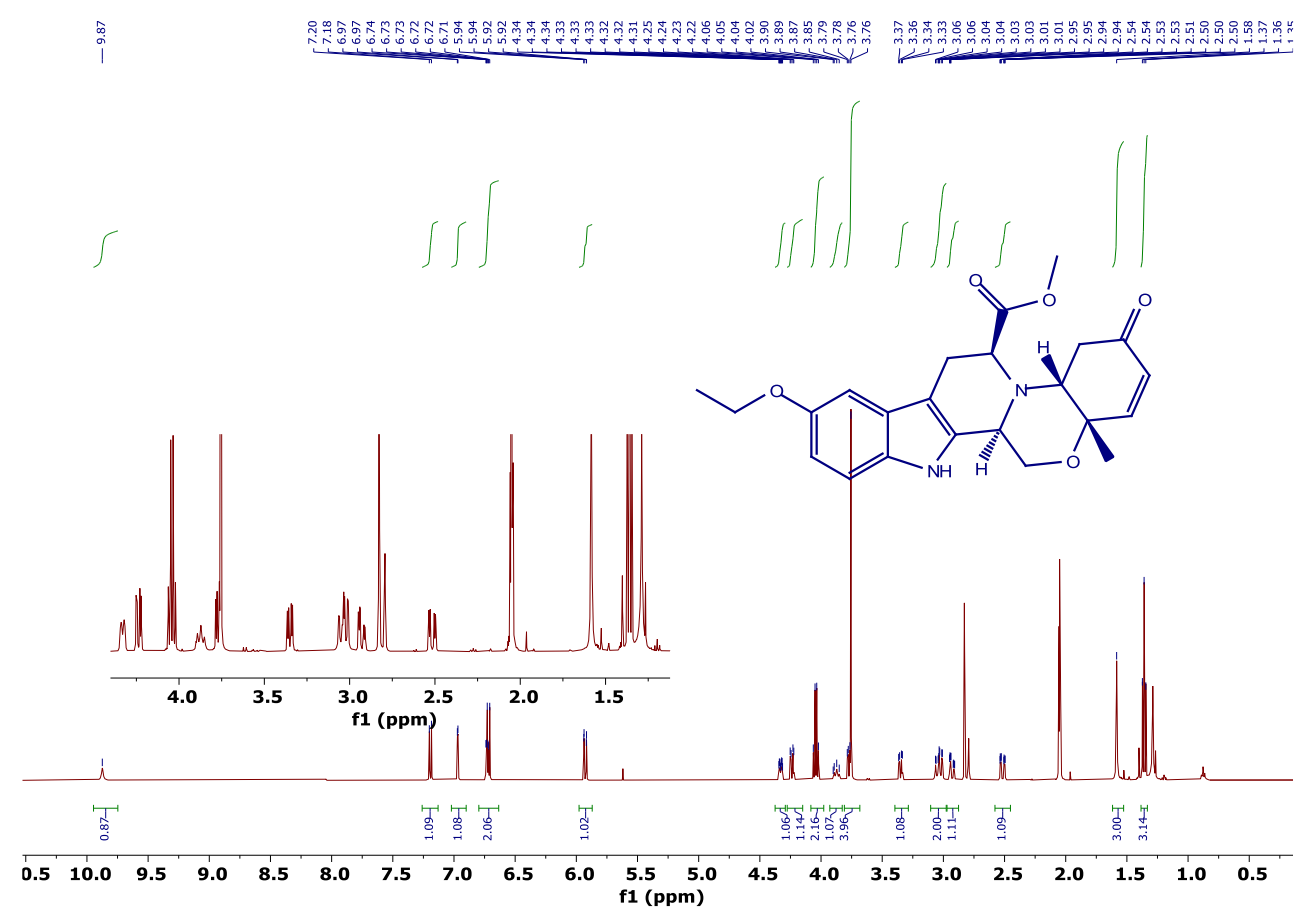

Figure S19 ${ }^{1} \mathrm{H}$ NMR (500 MHz, Acetone- $\mathrm{d}_{6}$ ) spectrum of methyl (2aS,6aR,8S,14bR)-11-ethoxy-2amethyl-5-oxo-2a,5,6,6a,8,9,14,14b-octahydro-1H-benzo[5',6'][1,4]oxazino[4',3':1,2]pyrido[3,4b]indole-8-carboxylate $(\mathbf{3 j})$

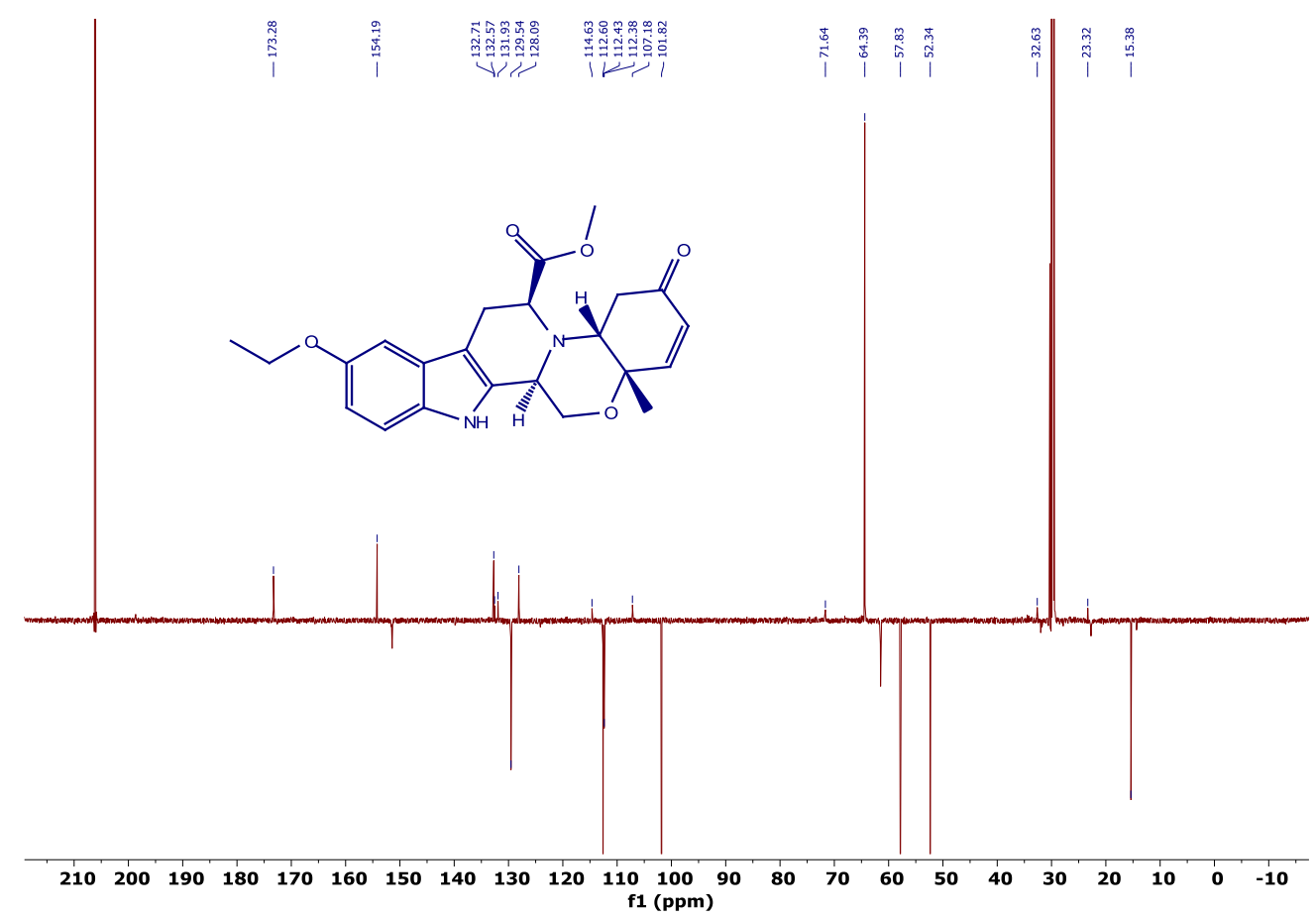

Figure S20 ${ }^{13} \mathrm{C}\left\{{ }^{1} \mathrm{H}\right\}$ APT NMR (125 MHz, Acetone- $\left.\mathrm{d}_{6}\right)$ spectrum of methyl (2aS,6aR,8S,14bR)-11ethoxy-2a-methyl-5-oxo-2a,5,6,6a,8,9,14,14b-octahydro-1Hbenzo[5',6'][1,4] oxazino[4',3':1,2]pyrido[3,4-b]indole-8-carboxylate (3j) 


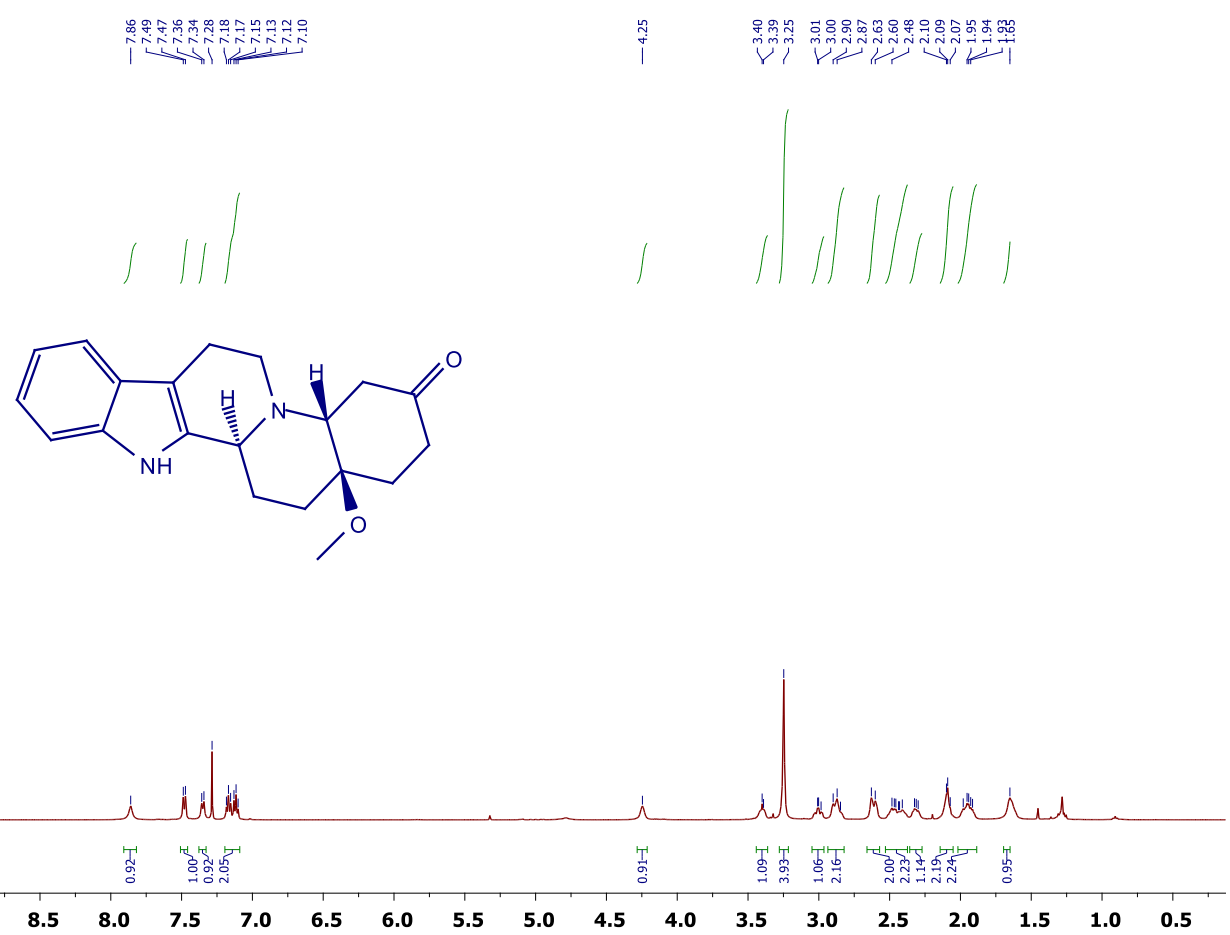

Figure S21 ${ }^{1} \mathrm{H}$ NMR $\left(500 \mathrm{MHz}, \mathrm{CDCl}_{3}\right)$ spectrum of (2aR,6aR,14bS)-2a-methoxy2,2a,3,4,6,6a,8,9,14,14b-decahydroindolo[2',3':3,4]pyrido[1,2-a]quinolin-5(1H)-one (4a)

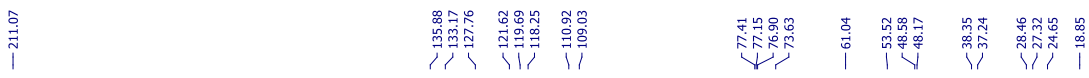
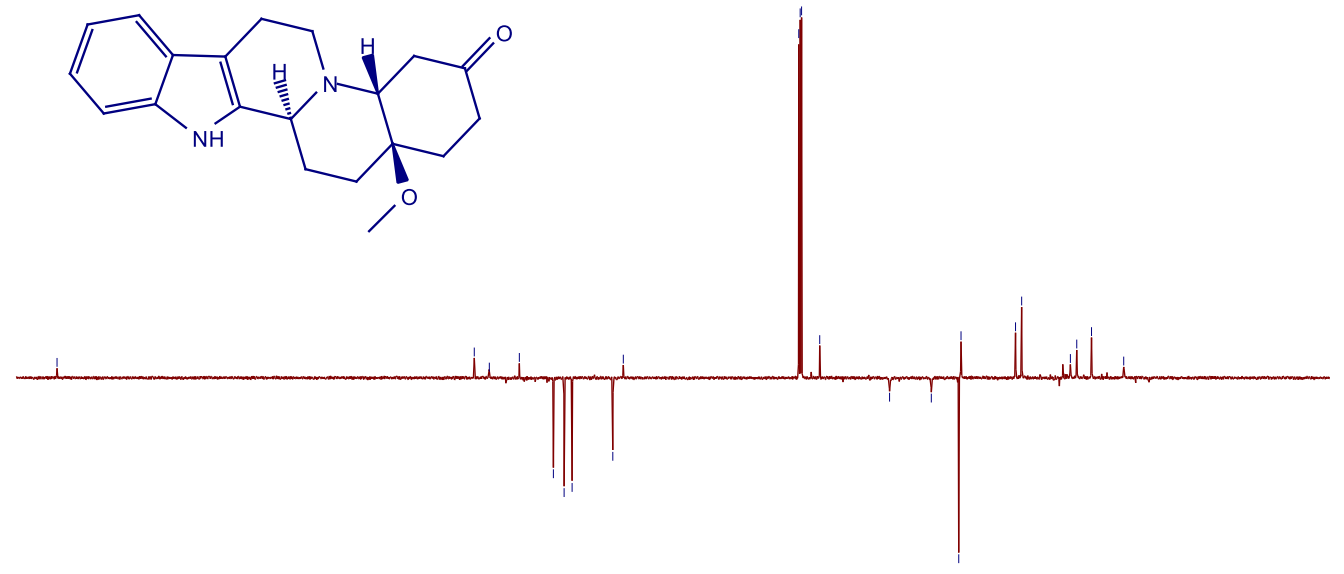

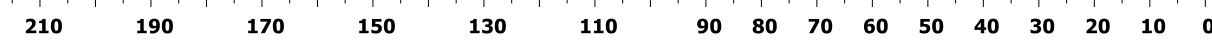

Figure S22 ${ }^{13} \mathrm{C}\left\{{ }^{1} \mathrm{H}\right\}$ APT NMR (125 MHz, $\left.\mathrm{CDCl}_{3}\right)$ spectrum of (2aR,6aR,14bS)-2a-methoxy2,2a,3,4,6,6a, 8,9,14,14b-decahydroindolo[2',3':3,4]pyrido[1,2-a]quinolin-5(1H)-one (4a) 


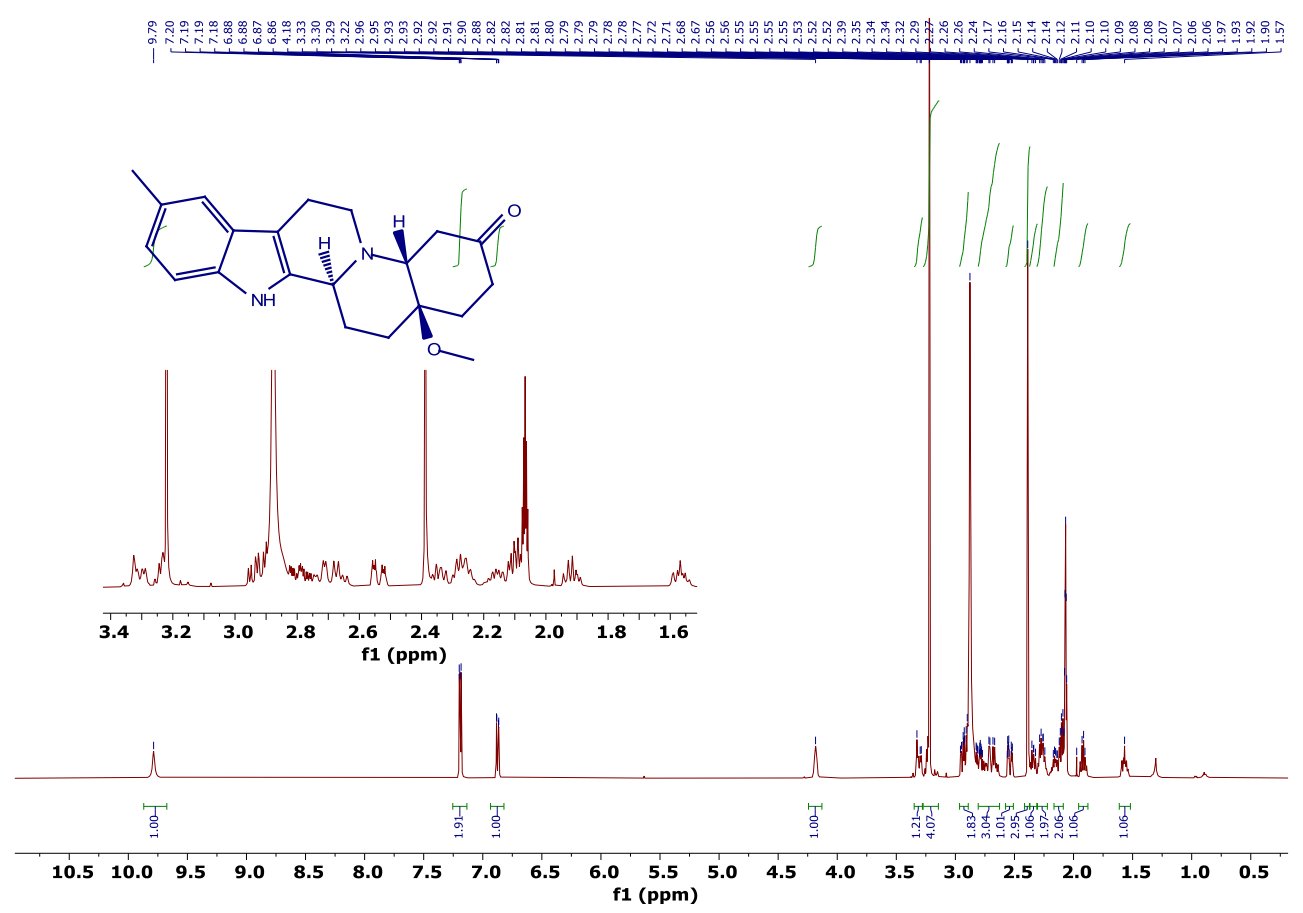

Figure S23 ${ }^{1} \mathrm{H}$ NMR $\left(500 \mathrm{MHz}\right.$, Acetone- $\left.\mathrm{d}_{6}\right)$ spectrum of (2aR,6aR,14bS)-2a-methoxy-11-methyl2,2a,3,4,6,6a,8,9,14,14b-decahydroindolo[2',3':3,4]pyrido[1,2-a]quinolin-5(1H)-one (4b)

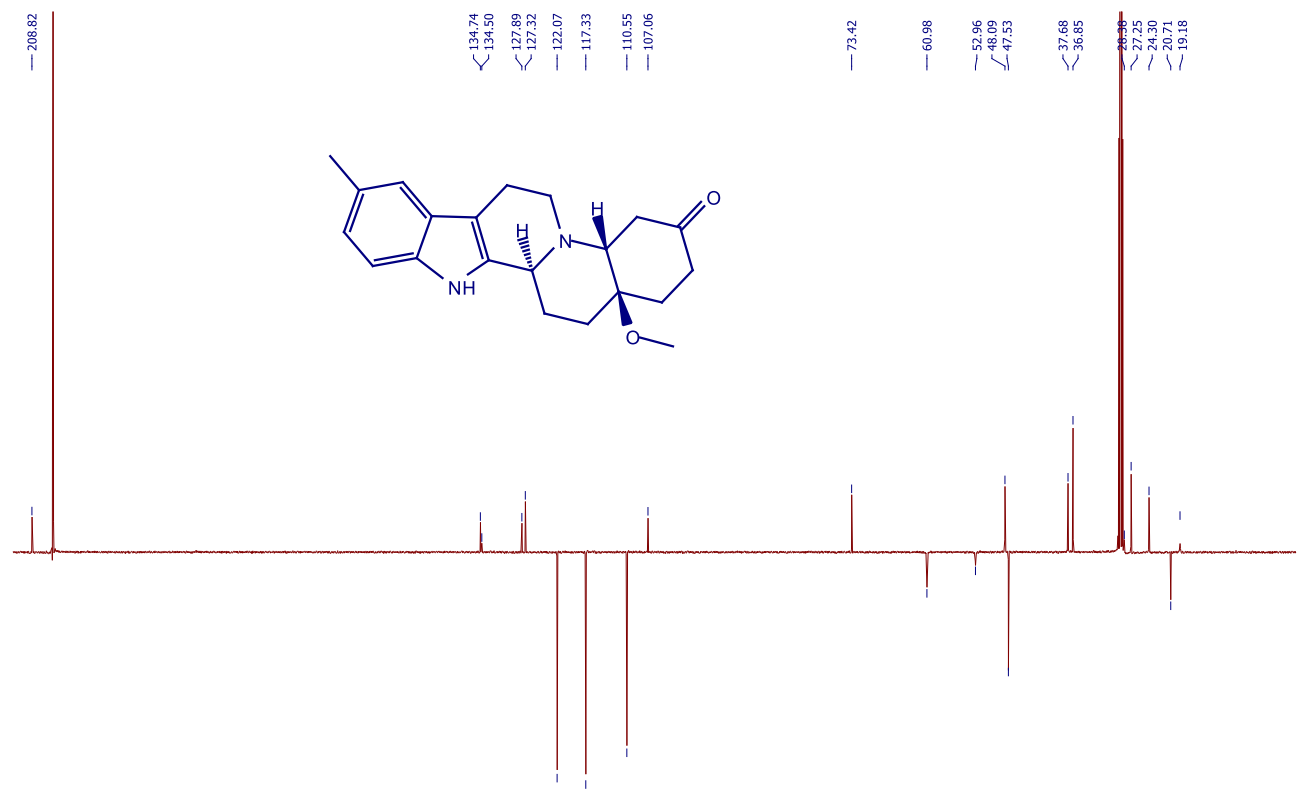

$\begin{array}{lllllllllllllllllllll}210 & 200 & 190 & 180 & 170 & 160 & 150 & 140 & 130 & 120 & \begin{array}{l}110 \\ \text { f1 (ppm) }\end{array} & 90 & 80 & 70 & 60 & 50 & 40 & 30 & 20 & 10\end{array}$

Figure S24 ${ }^{13} \mathrm{C}\left\{{ }^{1} \mathrm{H}\right\}$ APT NMR (125 MHz, Acetone-d $\left.\mathrm{d}_{6}\right)$ spectrum of (2aR,6aR,14bS)-2a-methoxy11-methyl-2,2a,3,4,6,6a,8,9,14,14b-decahydroindolo[2',3':3,4]pyrido[1,2-a]quinolin-5(1H)-one (4b) 


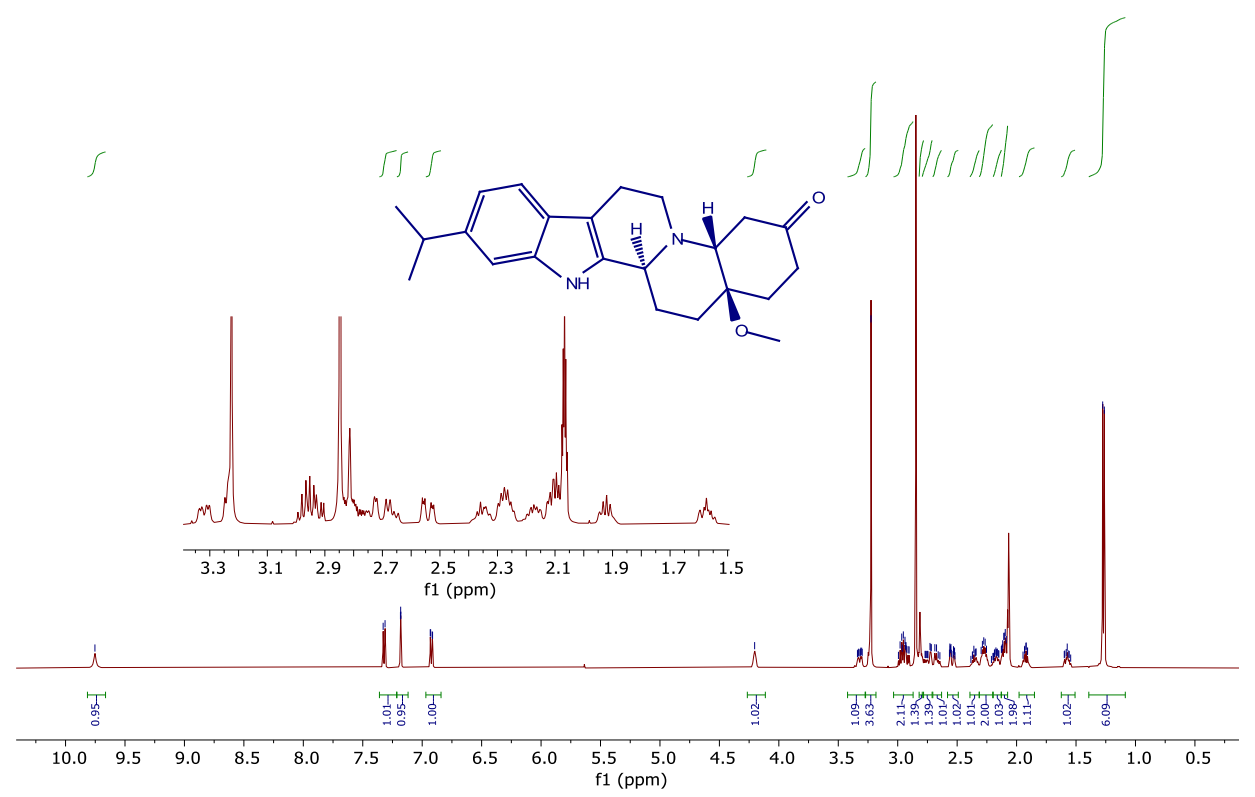

Figure S25 ${ }^{1} \mathrm{H}$ NMR (500 MHz, Acetone-d $)$ spectra of (2aR,6aR,14bS)-12-isopropyl-2a-methoxy2,2a,3,4,6,6a,8,9,14,14b-decahydroindolo[2',3':3,4]pyrido[1,2-a]quinolin-5(1H)-one (4c)

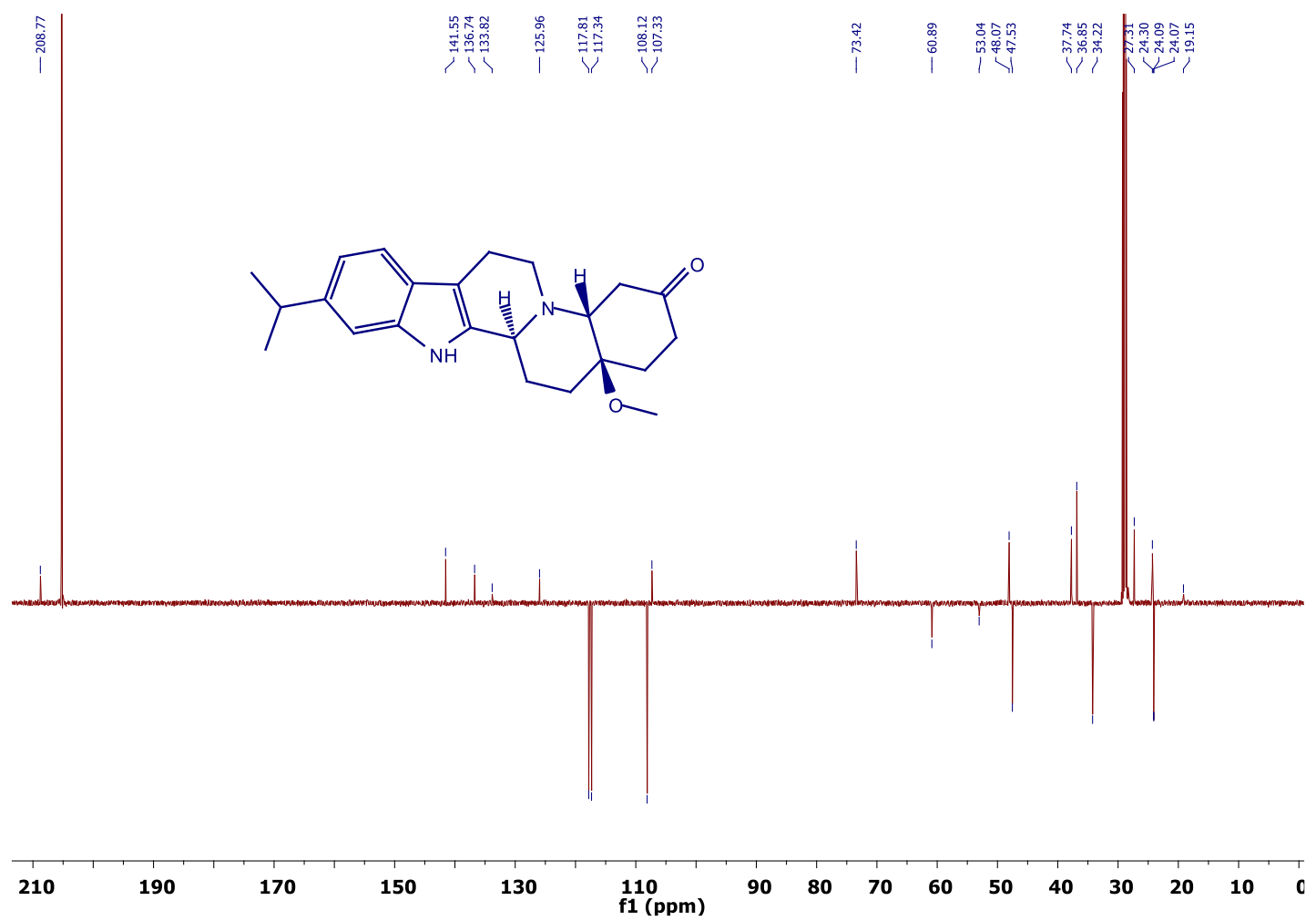

Figure S26 ${ }^{13} \mathrm{C}\left\{{ }^{1} \mathrm{H}\right\}$ APT NMR (125 MHz, Acetone-d 6 ) spectra of (2aR,6aR,14bS)-12-isopropyl-2amethoxy-2,2a,3,4,6,6a,8,9,14,14b-decahydroindolo[2',3':3,4]pyrido[1,2-a]quinolin-5(1H)-one $(4 \mathrm{c})$ 


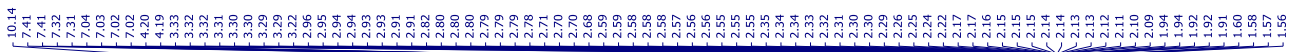

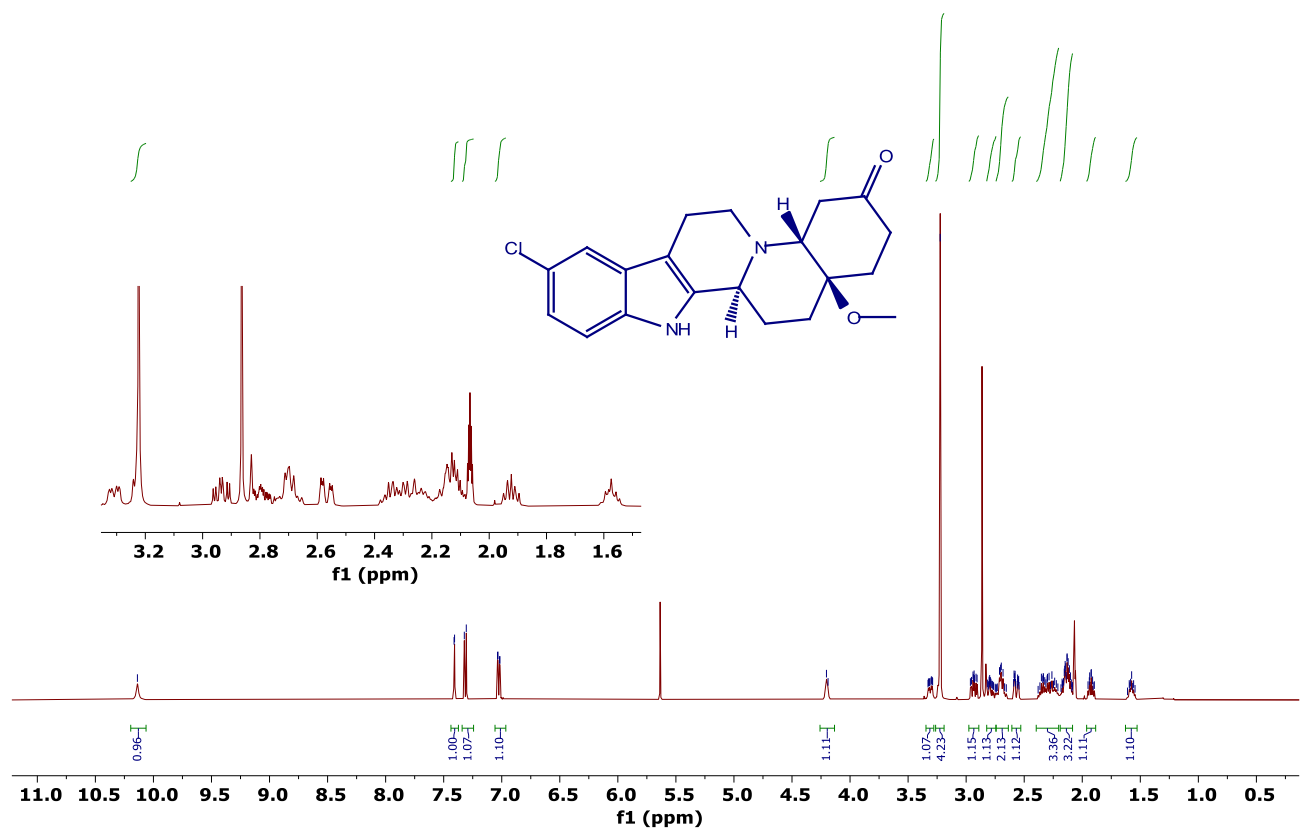

Figure S27 ${ }^{1} \mathrm{H}$ NMR (500 MHz, Acetone- $\mathrm{d}_{6}$ ) spectra of (2aR,6aR,14bS)-11-chloro-2a-methoxy2,2a,3,4,6,6a,8,9,14,14b-decahydroindolo[2',3':3,4]pyrido[1,2-a]quinolin-5(1H)-one (4d)
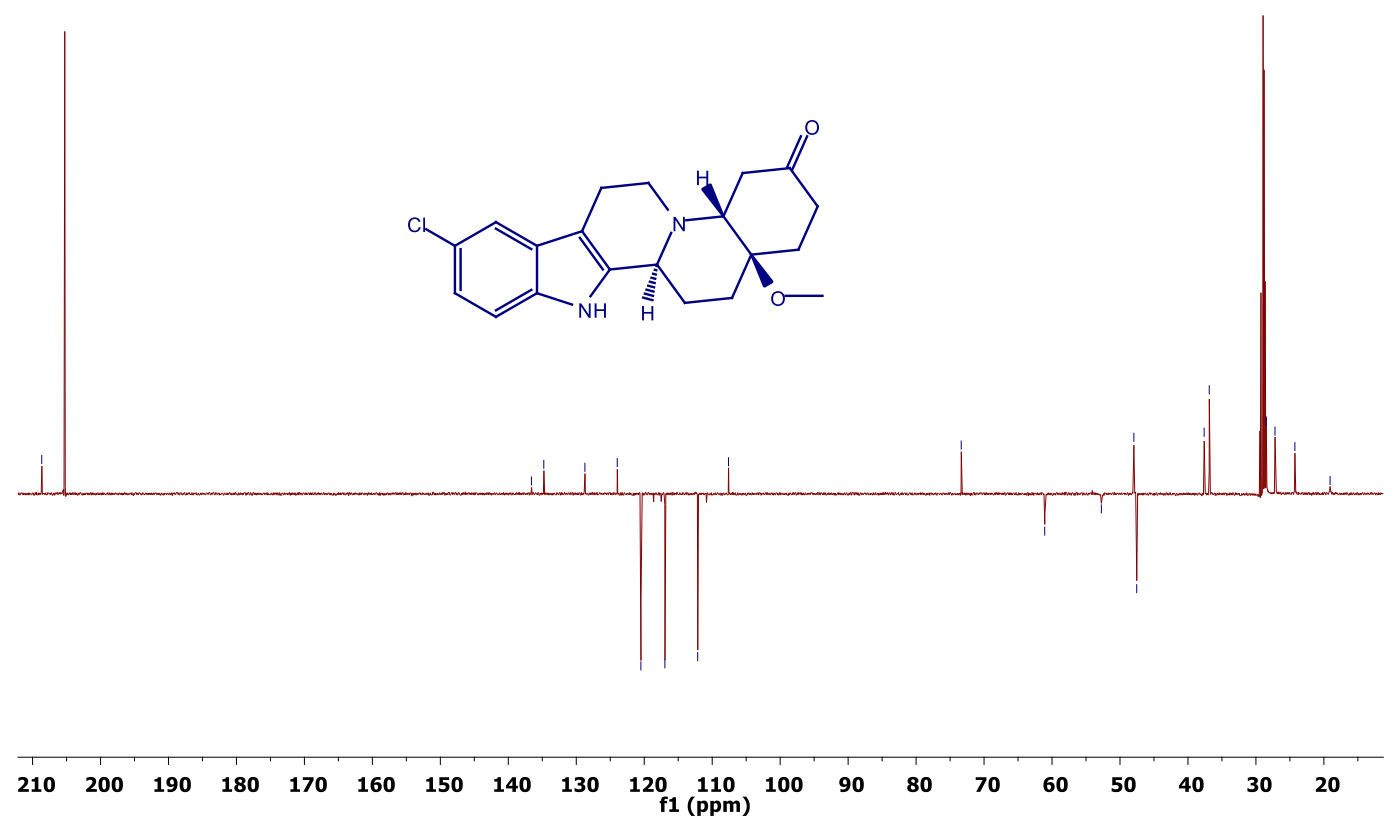

Figure S28 ${ }^{13} \mathrm{C}\left\{{ }^{1} \mathrm{H}\right\}$ APT NMR $\left(125 \mathrm{MHz}\right.$, Acetone- $\left.\mathrm{d}_{6}\right)$ spectra of (2aR,6aR, 14bS)-11-chloro-2amethoxy-2,2a,3,4,6,6a,8,9,14,14b-decahydroindolo[2',3':3,4]pyrido[1,2-a]quinolin-5(1H)-one (4d) 


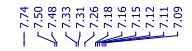
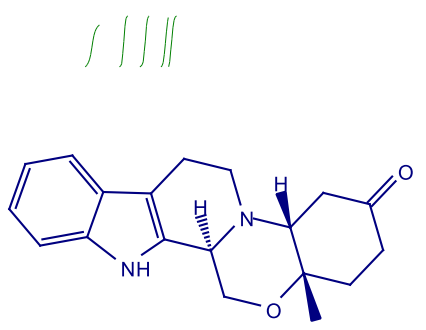
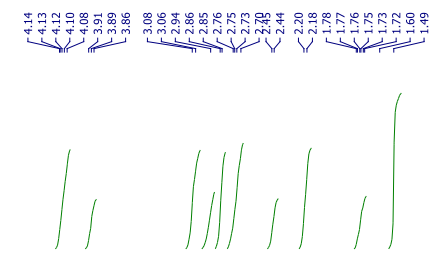

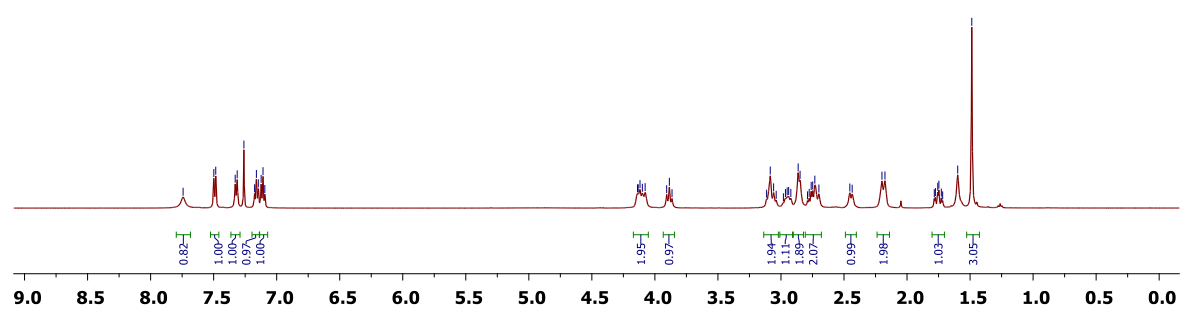

Figure S29 ${ }^{1} \mathrm{H}$ NMR $\left(500 \mathrm{MHz}, \mathrm{CDCl}_{3}\right)$ spectrum of $(2 \mathrm{aS}, 6 \mathrm{aR}, 14 \mathrm{bR})-2 \mathrm{a}-m e t h y l-$

3,4,6,6a,8,9,14,14b-octahydro-1H-benzo[5',6'][1,4] oxazino[4',3':1,2]pyrido[3,4-b]indol-5(2aH)one $(7)$

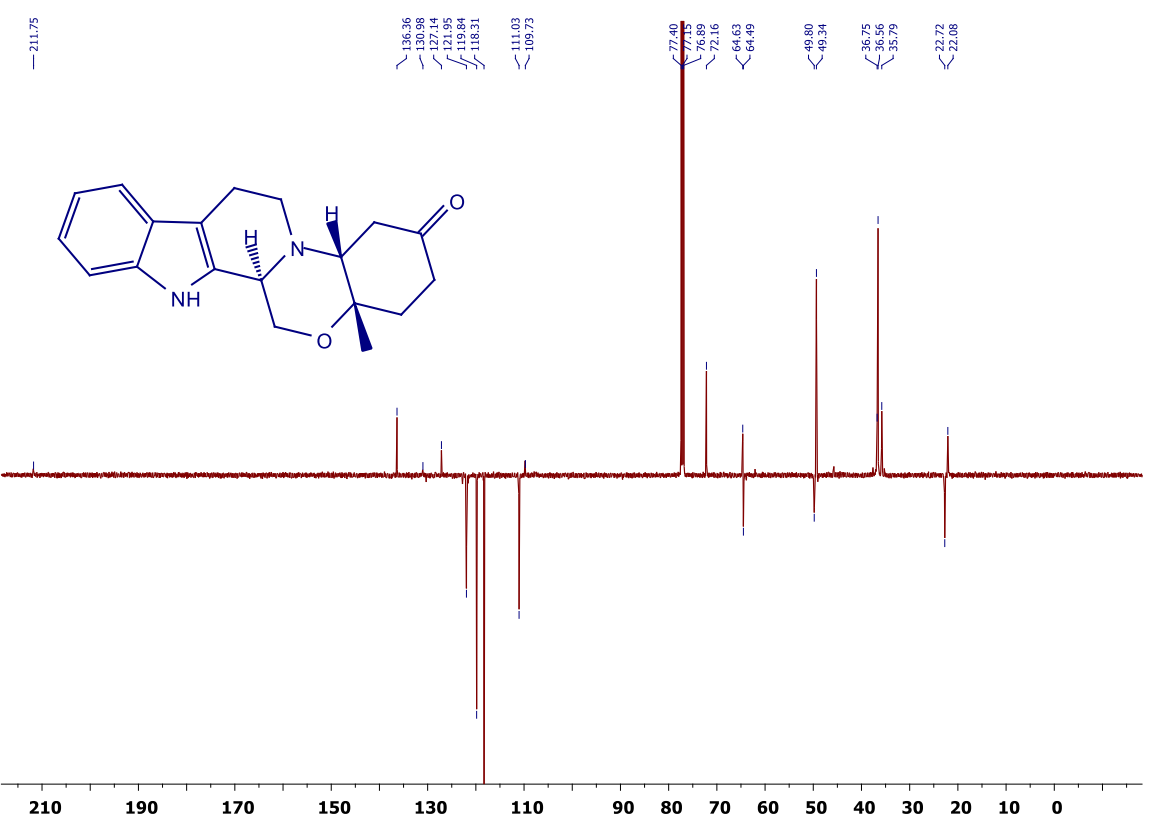

Figure S30 ${ }^{13} \mathrm{C}\left\{{ }^{1} \mathrm{H}\right\}$ APT NMR $\left(125 \mathrm{MHz}, \mathrm{CDCl}_{3}\right)$ spectrum of $(2 \mathrm{aS}, 6 \mathrm{aR}, 14 \mathrm{bR})-2 \mathrm{a}-\mathrm{methyl}-$ 3,4,6,6a, 8,9,14,14b-octahydro-1H-benzo[5',6'][1,4] oxazino[4',3':1,2]pyrido[3,4-b]indol-5(2aH)one $(7)$ 


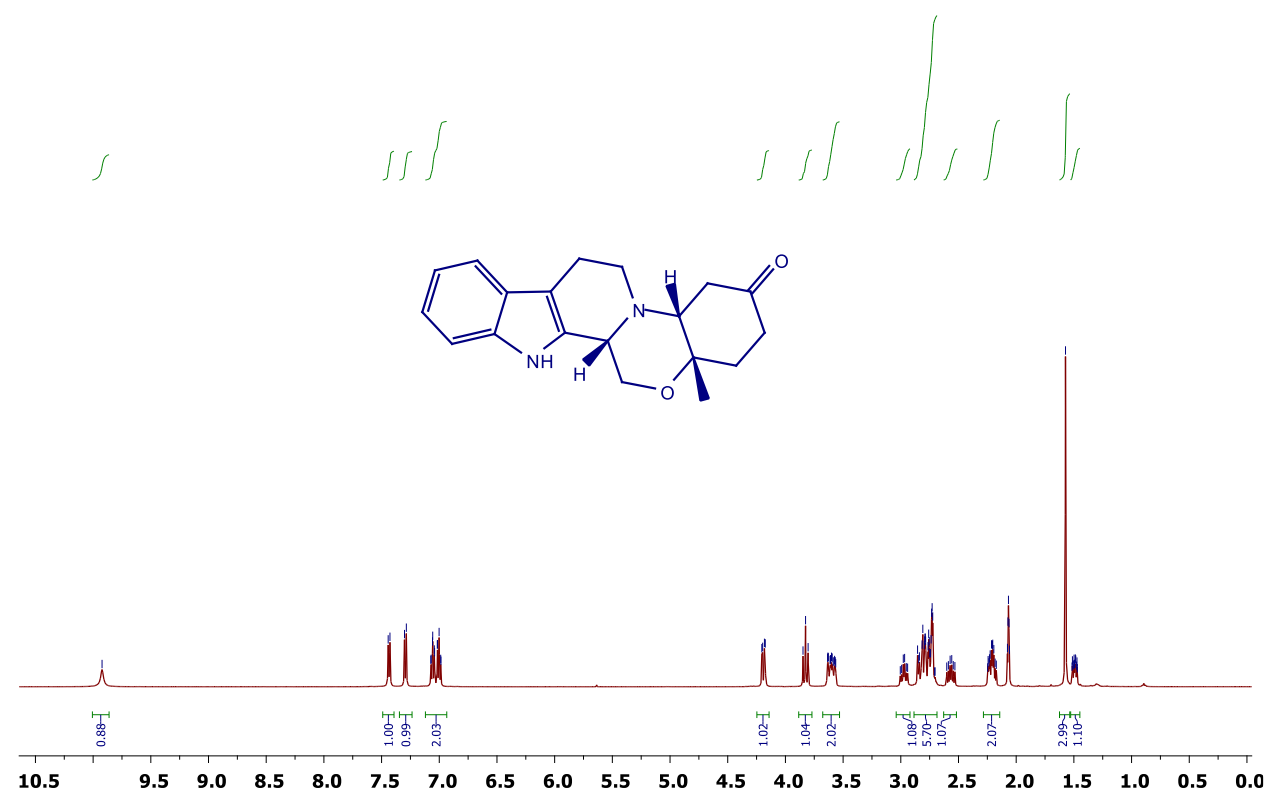

Figure S31 ${ }^{1} \mathrm{H}$ NMR $\left(500 \mathrm{MHz}\right.$, Acetone- $\left.\mathrm{d}_{6}\right)$ spectrum of $(2 \mathrm{aS}, 6 \mathrm{aR}, 14 \mathrm{bS})-2 \mathrm{a}-m e t h y l-$ 3,4,6,6a,8,9,14,14b-octahydro-1H-benzo[5',6'][1,4]oxazino[4',3':1,2]pyrido[3,4-b]indol-5(2aH)one (32)

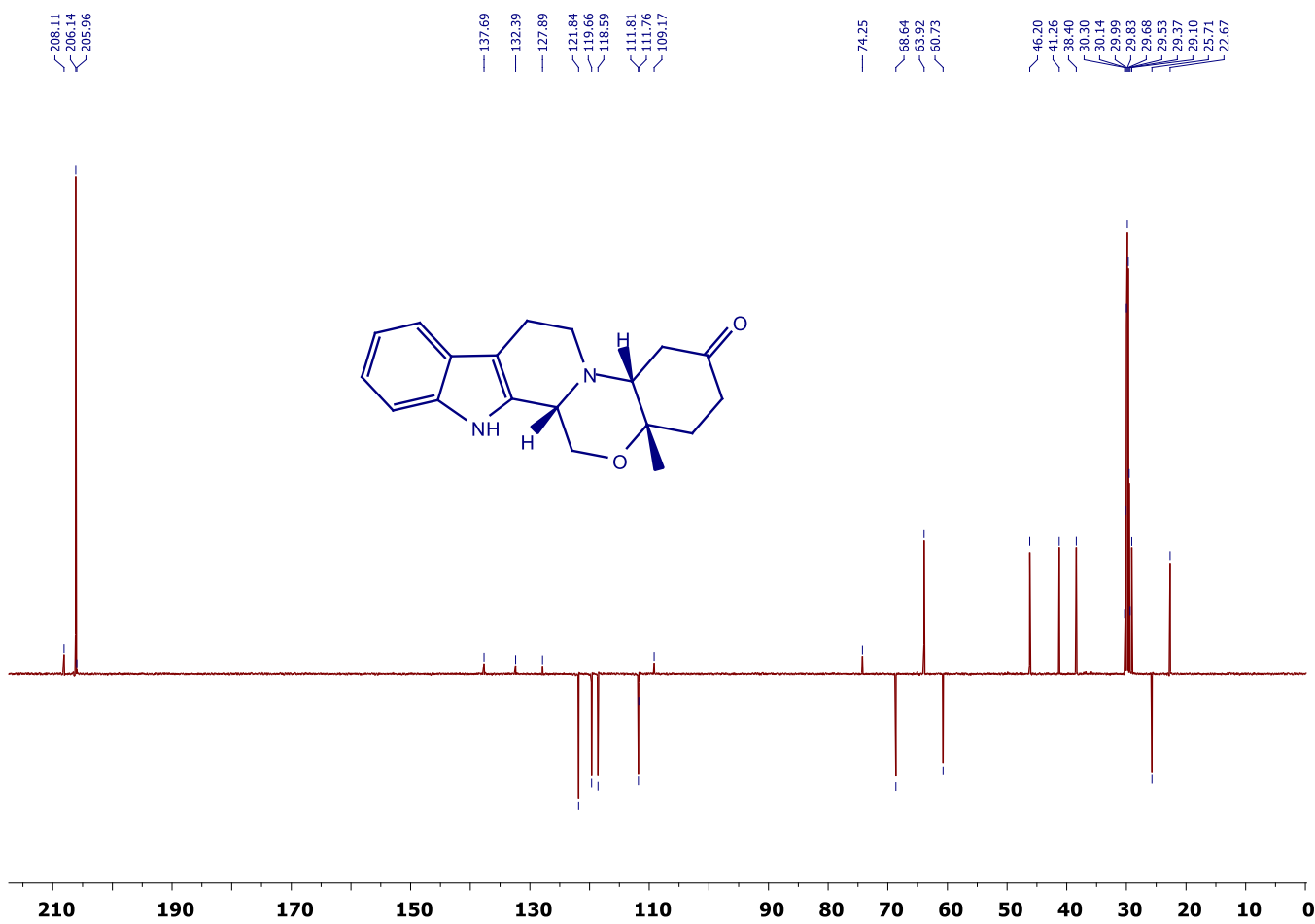

Figure S32 ${ }^{13} \mathrm{C}\left\{{ }^{1} \mathrm{H}\right\}$ APT (125 MHz, Acetone- $\left.\mathrm{d}_{6}\right)$ spectrum of $(2 \mathrm{aS}, 6 \mathrm{aR}, 14 \mathrm{bS})-2 \mathrm{a}-\mathrm{methyl}-$ 3,4,6,6a,8,9,14,14b-octahydro-1H-benzo[5',6'][1,4]oxazino[4',3':1,2]pyrido[3,4-b]indol-5(2aH)one (32) 


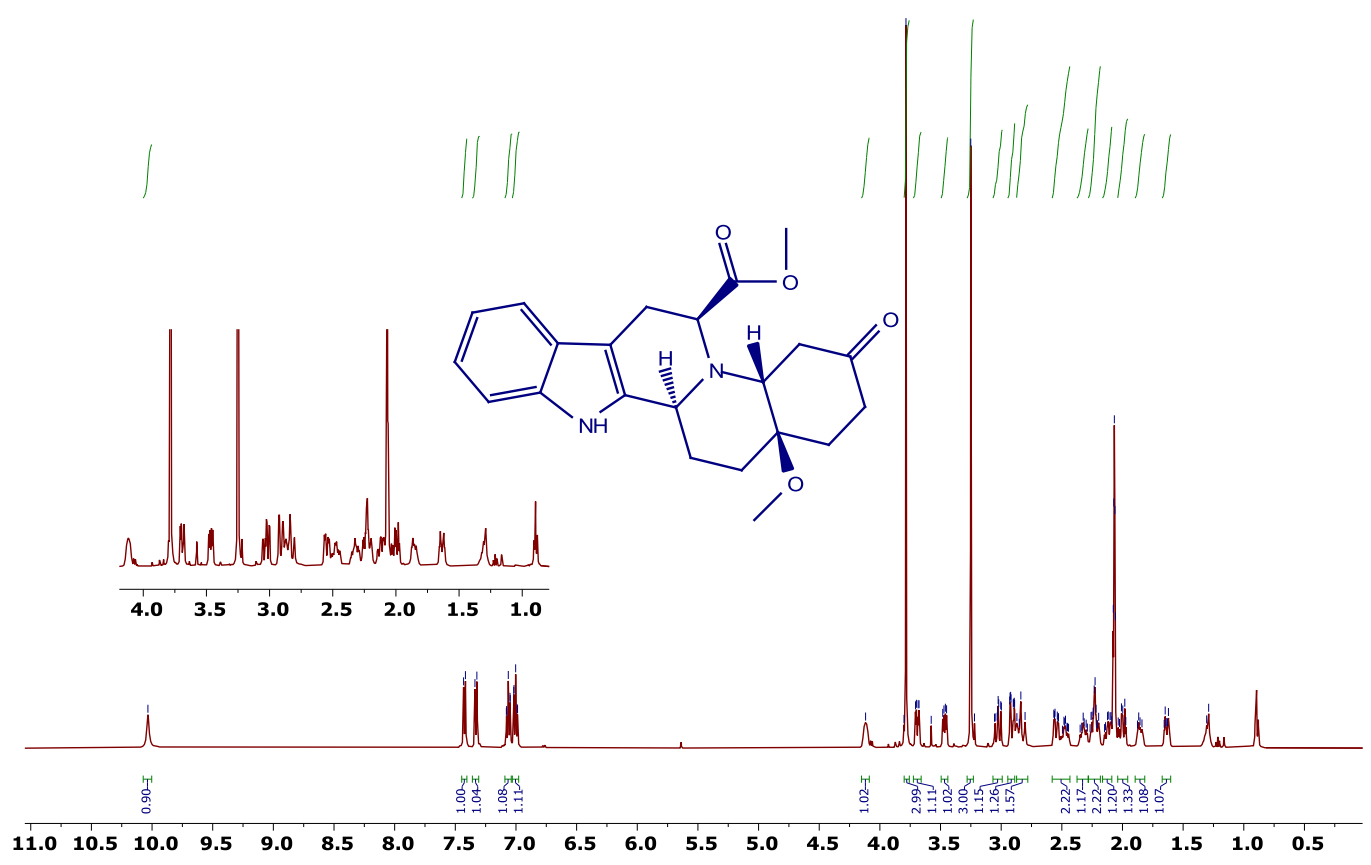

Figure S33 ${ }^{1} \mathrm{H}$ NMR (500 MHz, Acetone- $\mathrm{d}_{6}$ ) spectrum of methyl (2aR,6aR,8S,14bS)-2a-methoxy-5oxo-1,2,2a,3,4,5,6,6a,8,9,14,14b-dodecahydroindolo[2',3':3,4]pyrido[1,2-a]quinoline-8carboxylate (10a)

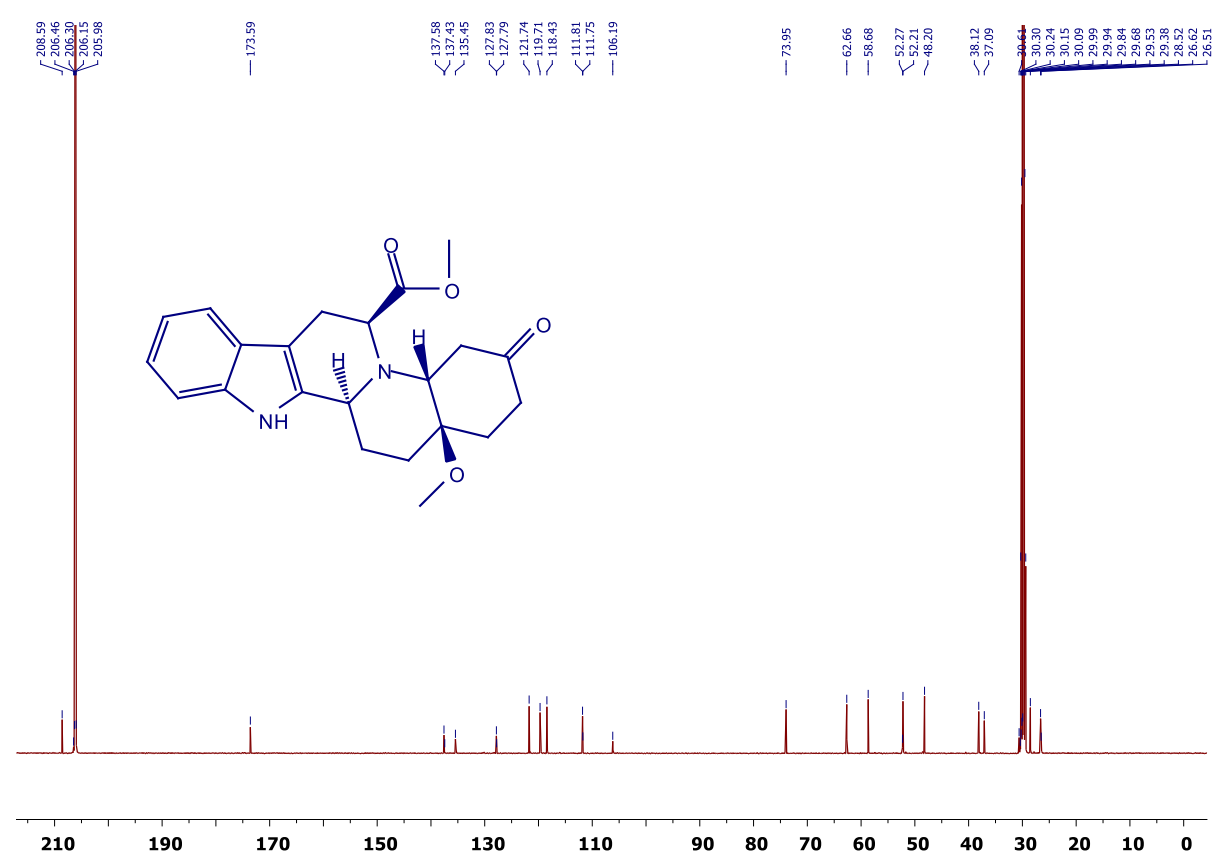

Figure S34 ${ }^{13} \mathrm{C}\left\{{ }^{1} \mathrm{H}\right\}$ NMR (125 MHz, Acetone-d $\mathrm{d}_{6}$ ) spectrum of methyl (2aR,6aR,8S,14bS)-2amethoxy-5-oxo-1,2,2a,3,4,5,6,6a,8,9,14,14b-dodecahydroindolo[2',3':3,4]pyrido[1,2-a]quinoline8-carboxylate $(\mathbf{1 0 a})$ 

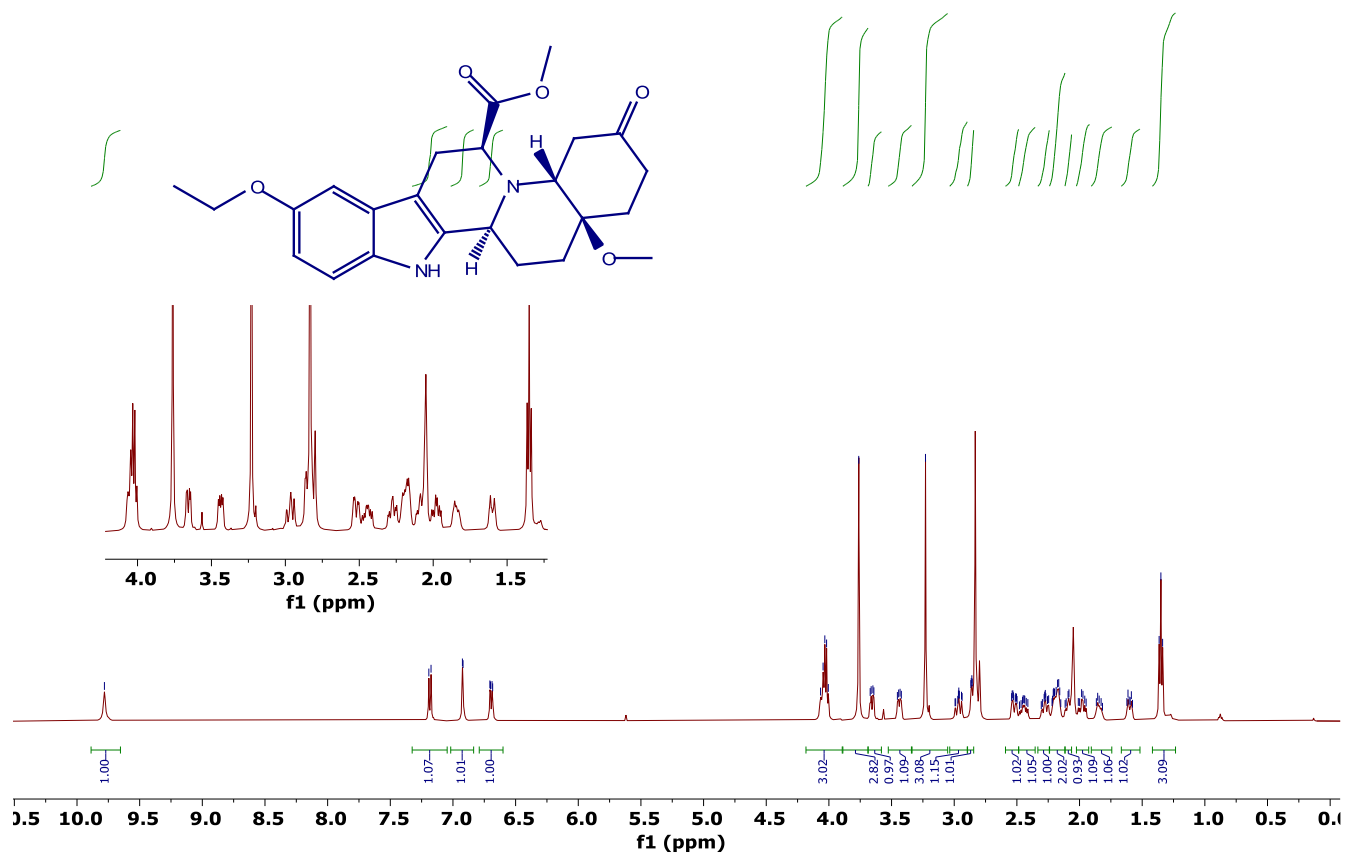

Figure S35 ${ }^{1} \mathrm{H}$ NMR (500 MHz, Acetone- $\mathrm{d}_{6}$ ) spectra of methyl (2aR,6aR,8S,14bS)-11-ethoxy-2amethoxy-5-oxo-1,2,2a,3,4,5,6,6a,8,9,14,14b-dodecahydroindolo[2',3':3,4]pyrido[1,2-a]quinoline8-carboxylate $(\mathbf{1 0 b})$

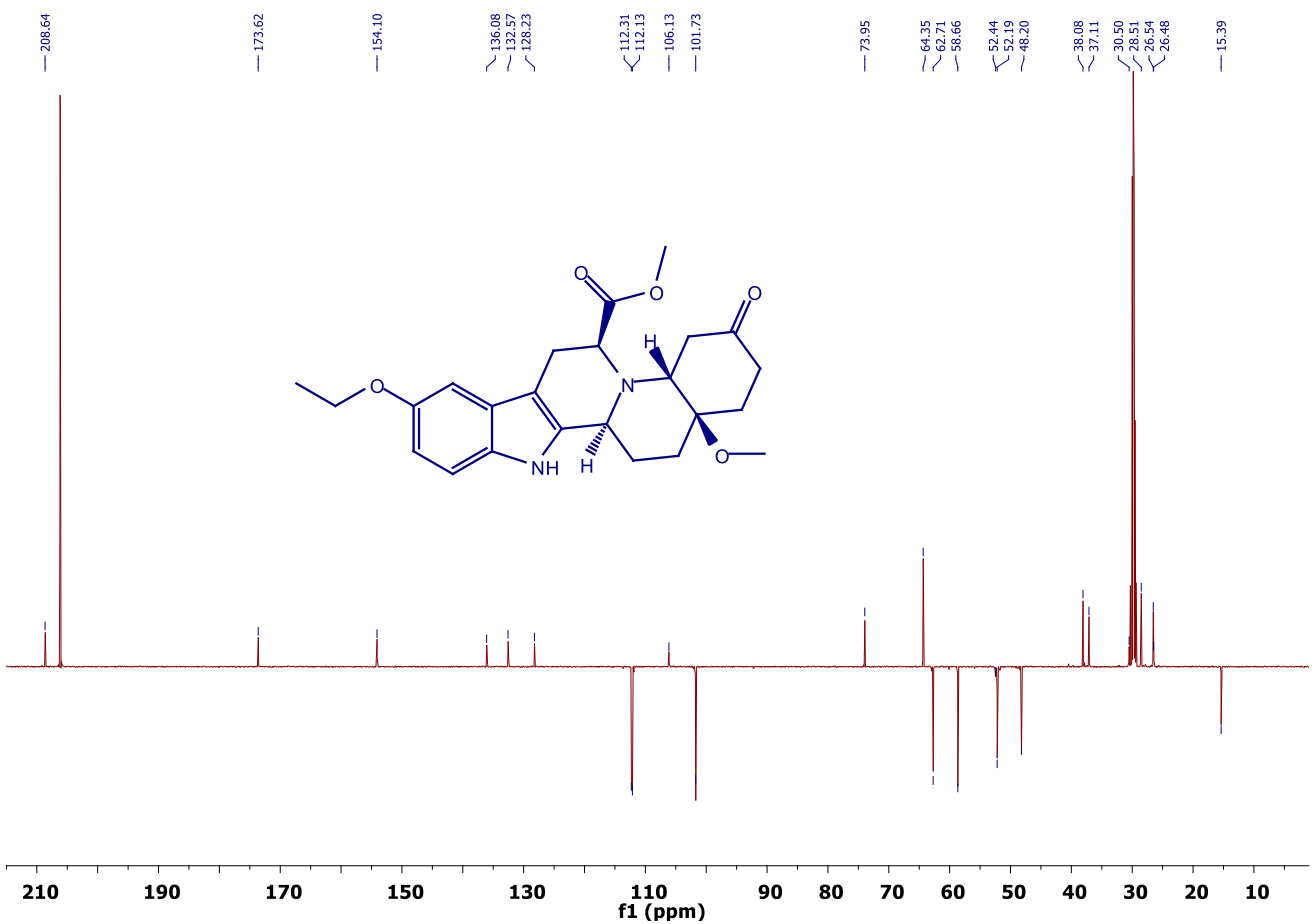

Figure S36 ${ }^{13} \mathrm{C}\left\{{ }^{1} \mathrm{H}\right\}$ APT NMR (125 MHz, Acetone-d $\left.\mathrm{d}_{6}\right)$ spectra of methyl (2aR,6aR,8S,14bS)-11ethoxy-2a-methoxy-5-oxo-1,2,2a,3,4,5,6,6a,8,9,14,14b-dodecahydroindolo[2',3':3,4]pyrido[1,2a]quinoline-8-carboxylate (10b) 


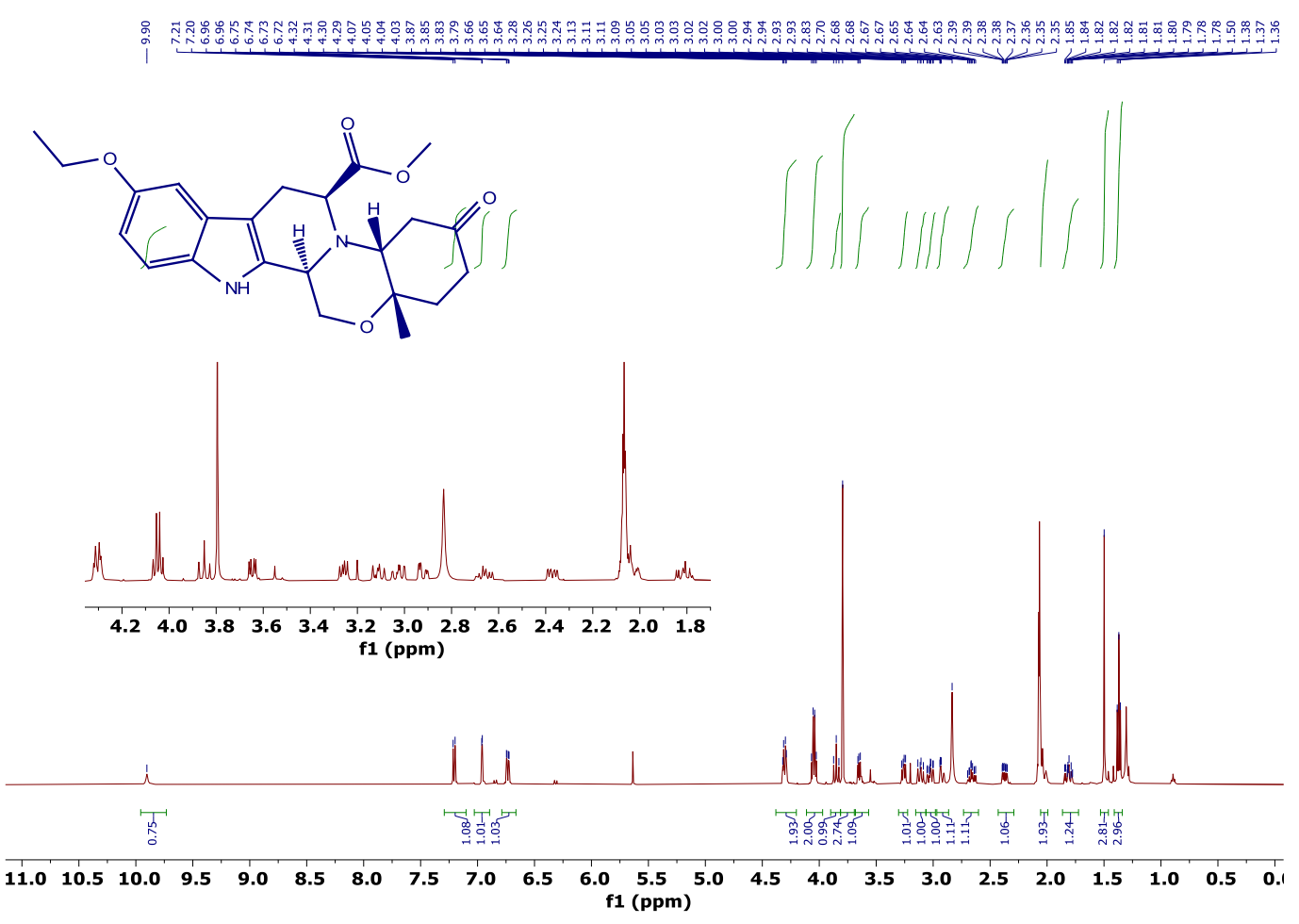

Figure S37 ${ }^{1} \mathrm{H}$ NMR (500 MHz, Acetone-d ${ }_{6}$ ) spectra of methyl (2aS,6aR,8S, 14bR)-11-ethoxy-2amethyl-5-oxo-2a,3,4,5,6,6a,8,9,14,14b-decahydro- $1 \mathrm{H}$ benzo[5',6'][1,4]oxazino[4',3':1,2]pyrido[3,4-b]indole-8-carboxylate (13)
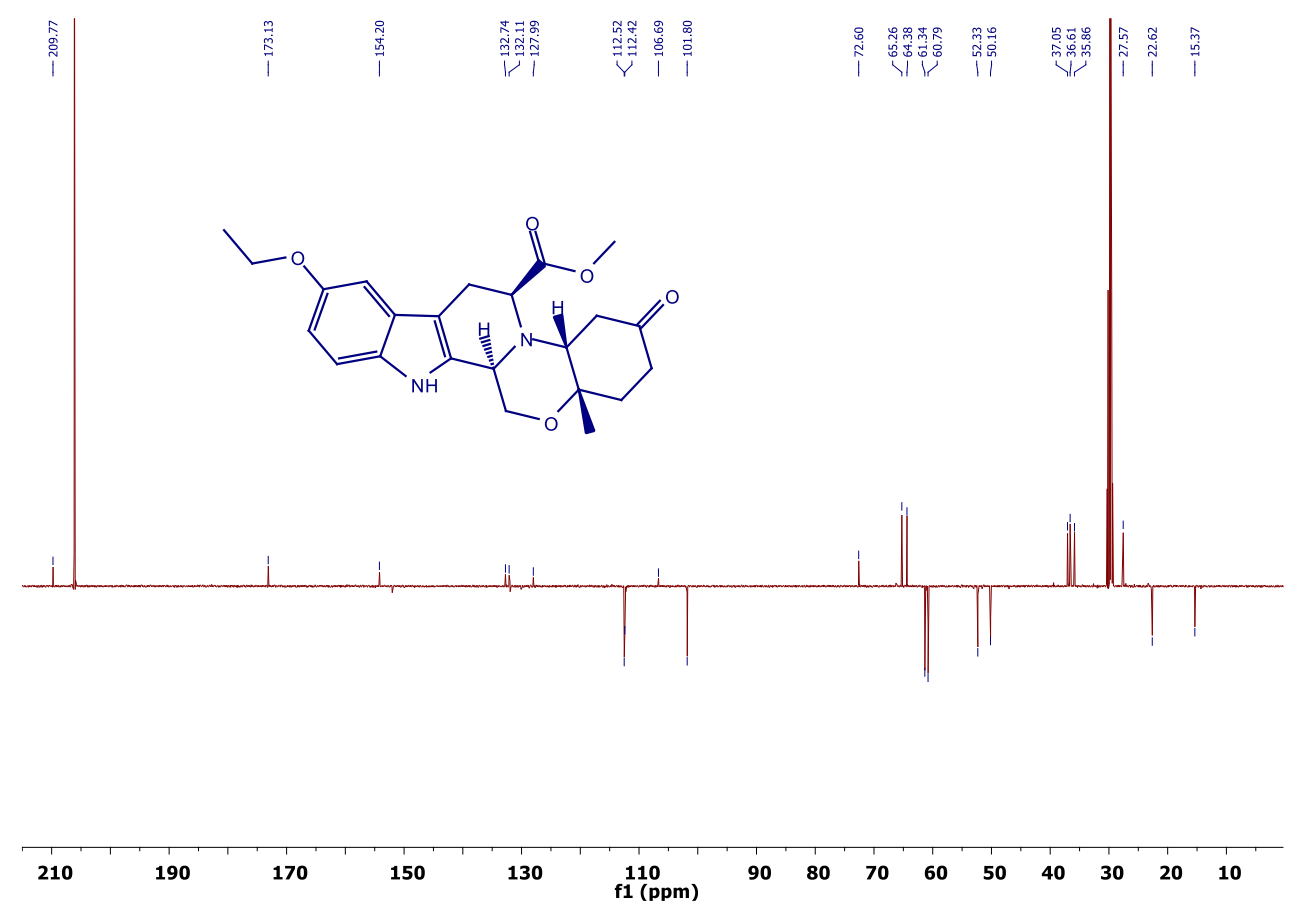

Figure S38 ${ }^{13} \mathrm{C}\left\{{ }^{1} \mathrm{H}\right\}$ APT NMR (125 MHz, Acetone-d $\left.\mathrm{d}_{6}\right)$ spectra of methyl (2aS,6aR,8S,14bR)-11ethoxy-2a-methyl-5-oxo-2a,3,4,5,6,6a,8,9,14,14b-decahydro-1Hbenzo[5',6'][1,4] oxazino[4',3':1,2]pyrido[3,4-b]indole-8-carboxylate (13) 


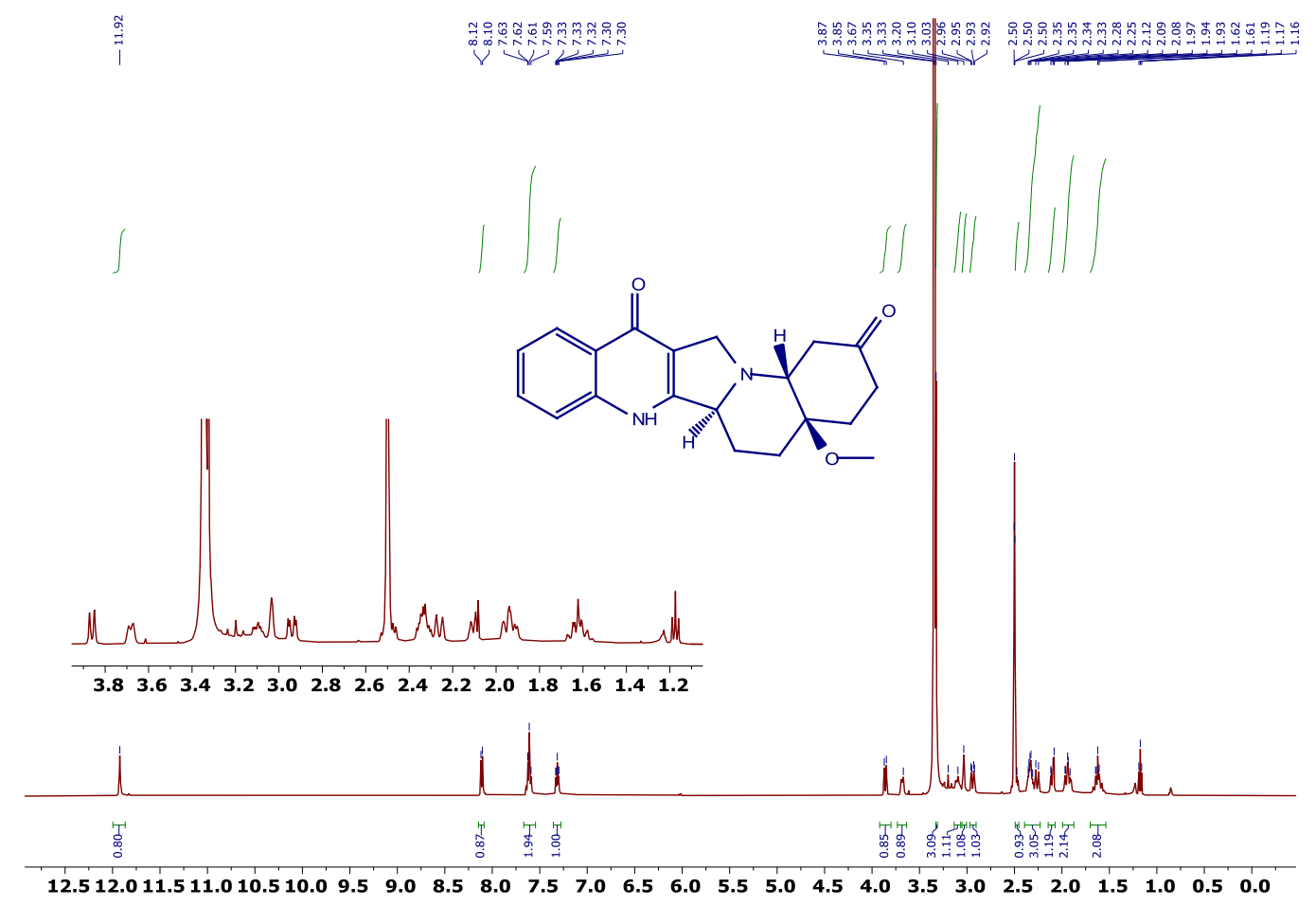

Figure S39 ${ }^{1} \mathrm{H}$ NMR (500 MHz, DMSO-d $\left.{ }_{6}\right)$ spectrum of (4aR,6aS, 14aR)-4a-methoxy1,3,4,4a,5,6,6a, 7,13,14a-decahydropyrrolo[1,2-a:3,4-b']diquinoline-2,12-dione (6a)
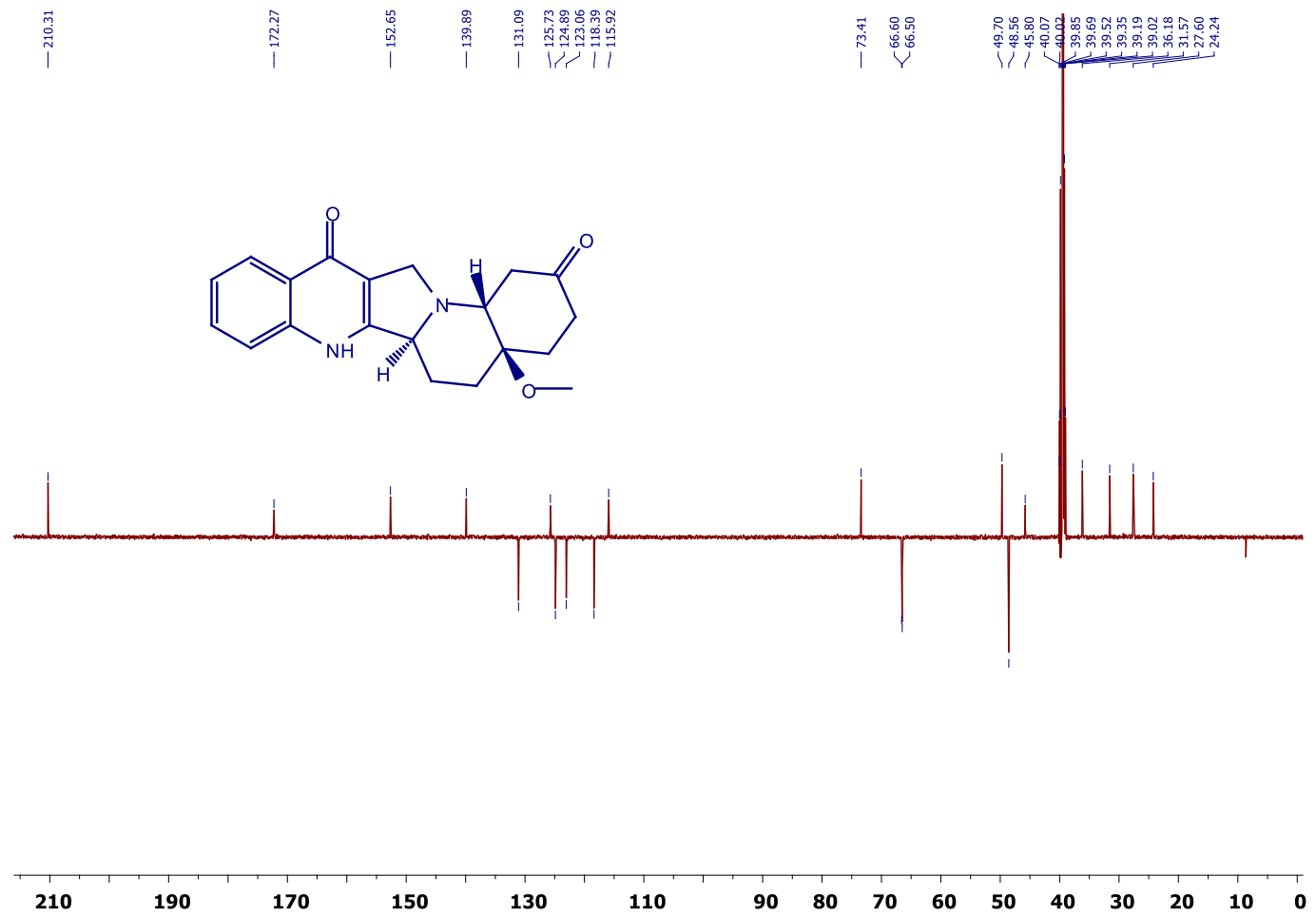

Figure S40 ${ }^{13} \mathrm{C}\left\{{ }^{1} \mathrm{H}\right\}$ APT NMR (125 MHz, DMSO-d $\left.\mathrm{d}_{6}\right)$ spectrum of (4aR,6aS,14aR)-4a-methoxy1,3,4,4a,5,6,6a, 7,13,14a-decahydropyrrolo[1,2-a:3,4-b']diquinoline-2,12-dione (6a) 

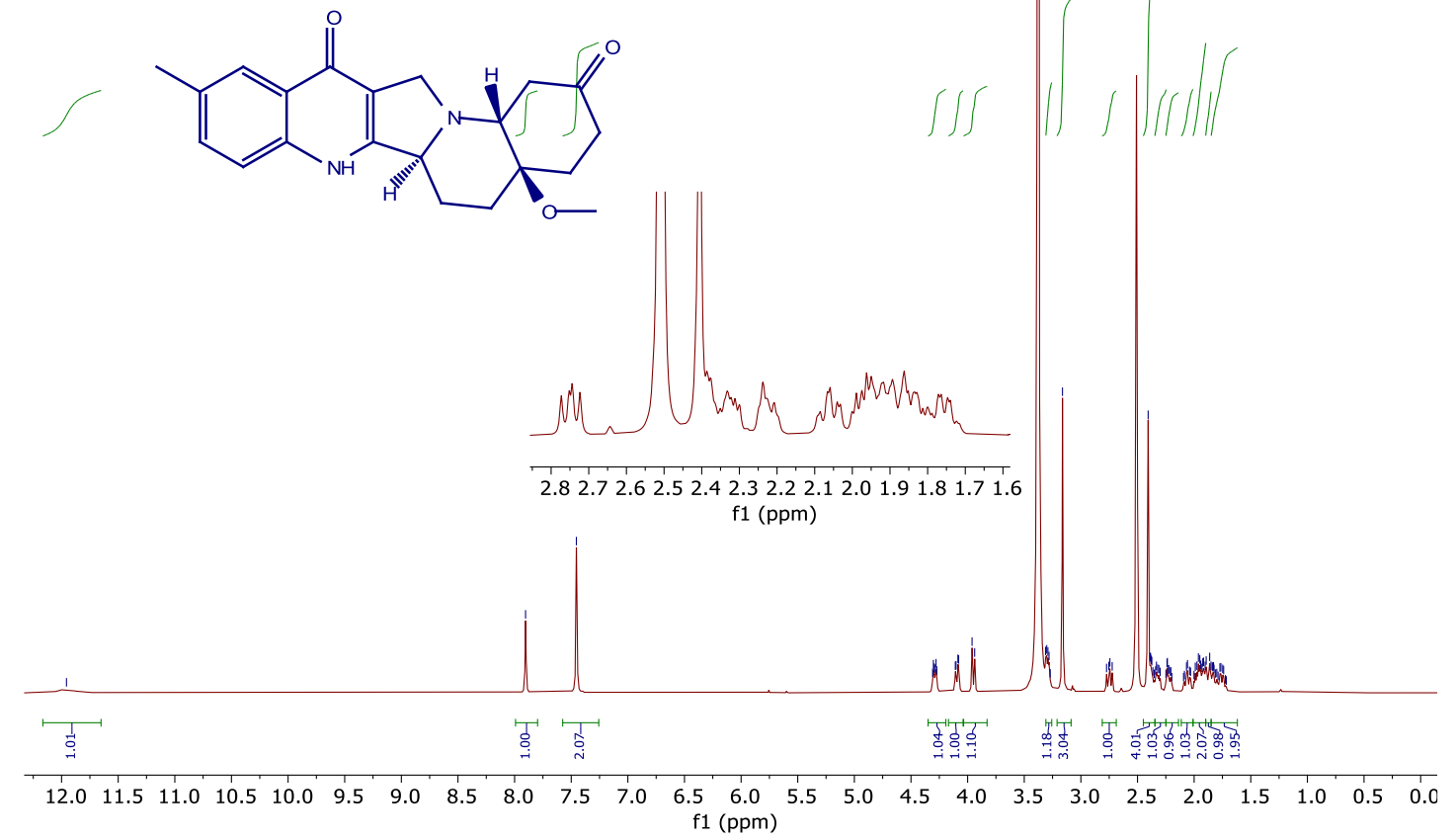

Figure S41 ${ }^{1} \mathrm{H}$ NMR (500 MHz, DMSO-d 6 ) spectrum of (4aR,6aS, 14aR)-4a-methoxy-10-methyl1,3,4,4a,5,6,6a, 7,13,14a-decahydropyrrolo[1,2-a:3,4-b']diquinoline-2,12-dione (6b)
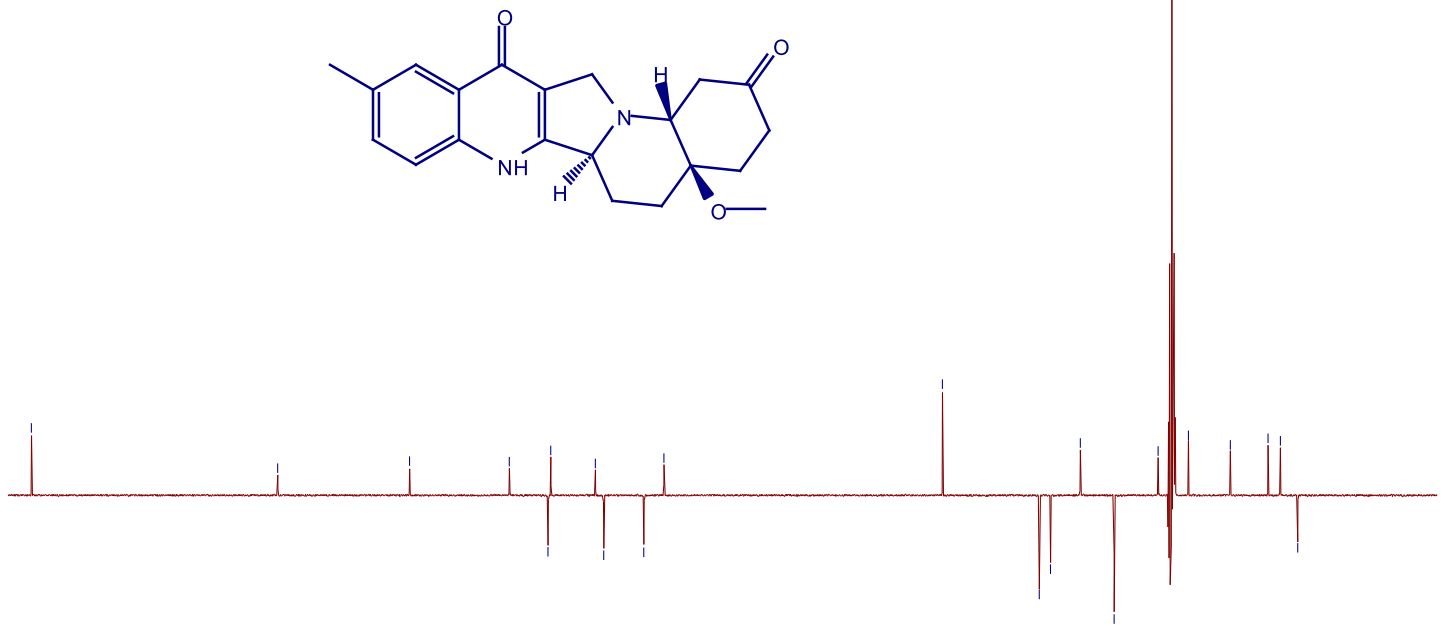

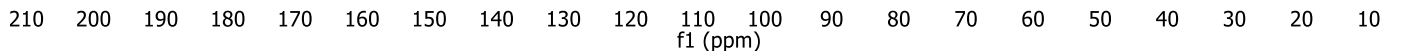

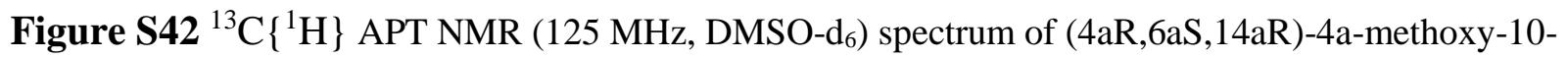
methyl-1,3,4,4a,5,6,6a,7,13,14a-decahydropyrrolo[1,2-a:3,4-b']diquinoline-2,12-dione (6b) 


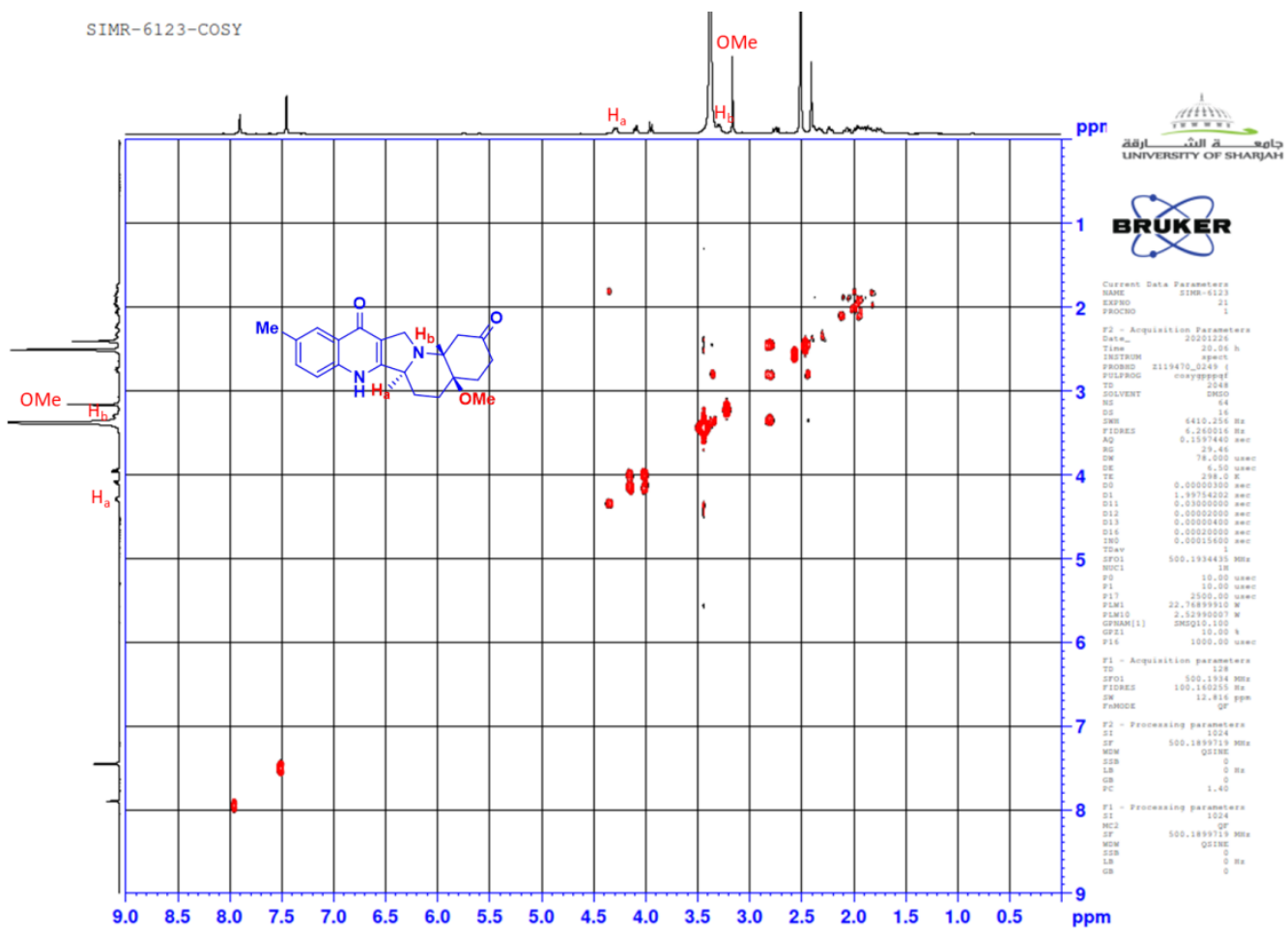

Figure S43 COSY NMR (500 MHz, DMSO-d 6 ) spectrum of (4aR,6aS,14aR)-4a-methoxy-10-methyl1,3,4,4a,5,6,6a, 7,13,14a-decahydropyrrolo[1,2-a:3,4-b']diquinoline-2,12-dione (6b) 


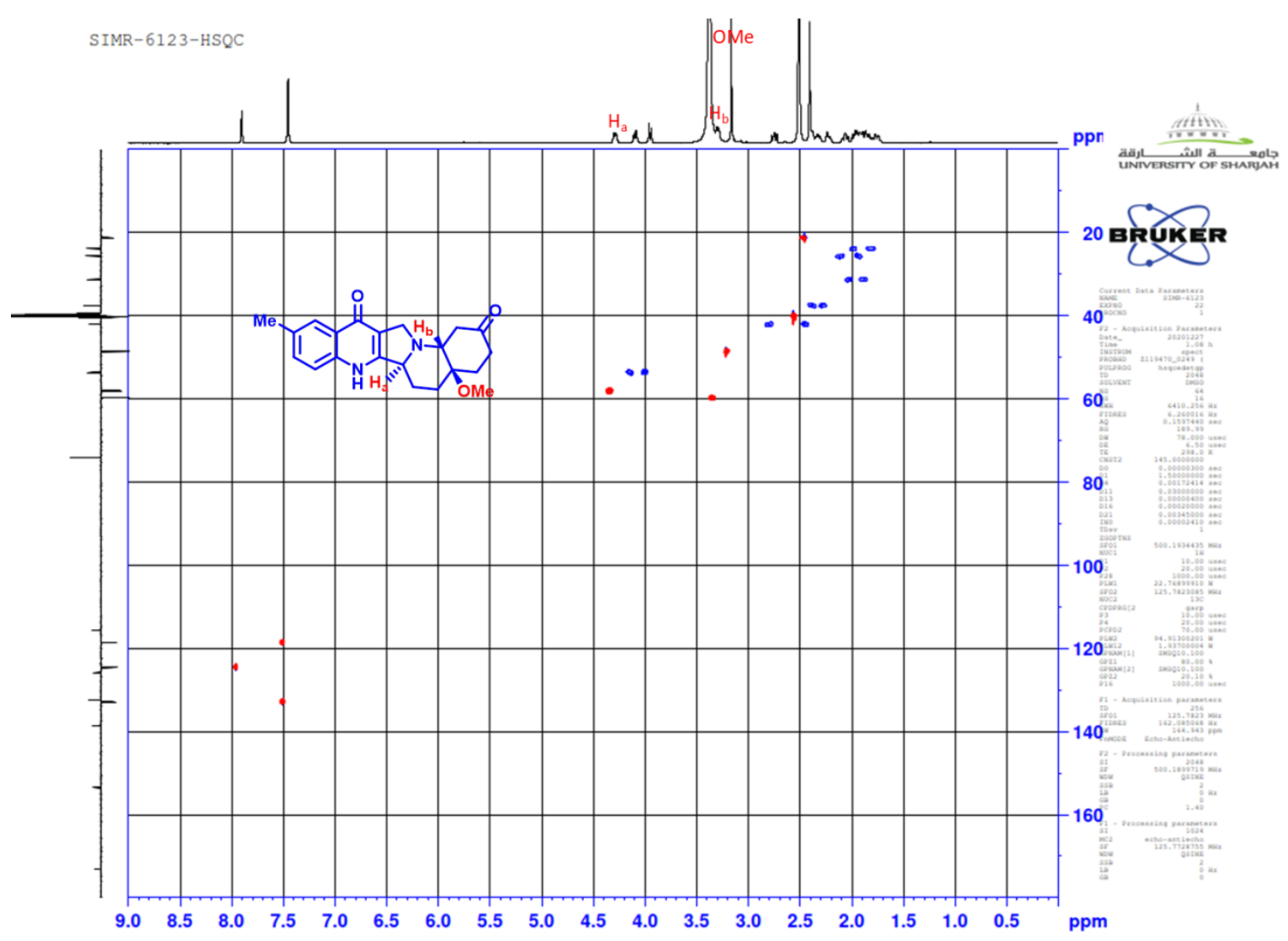

Figure S44 HSQC NMR (500 MHz, DMSO-d ${ }_{6}$ ) spectrum of (4aR,6aS, 14aR)-4a-methoxy-10-methyl1,3,4,4a,5,6,6a,7,13,14a-decahydropyrrolo[1,2-a:3,4-b']diquinoline-2,12-dione (6b) 


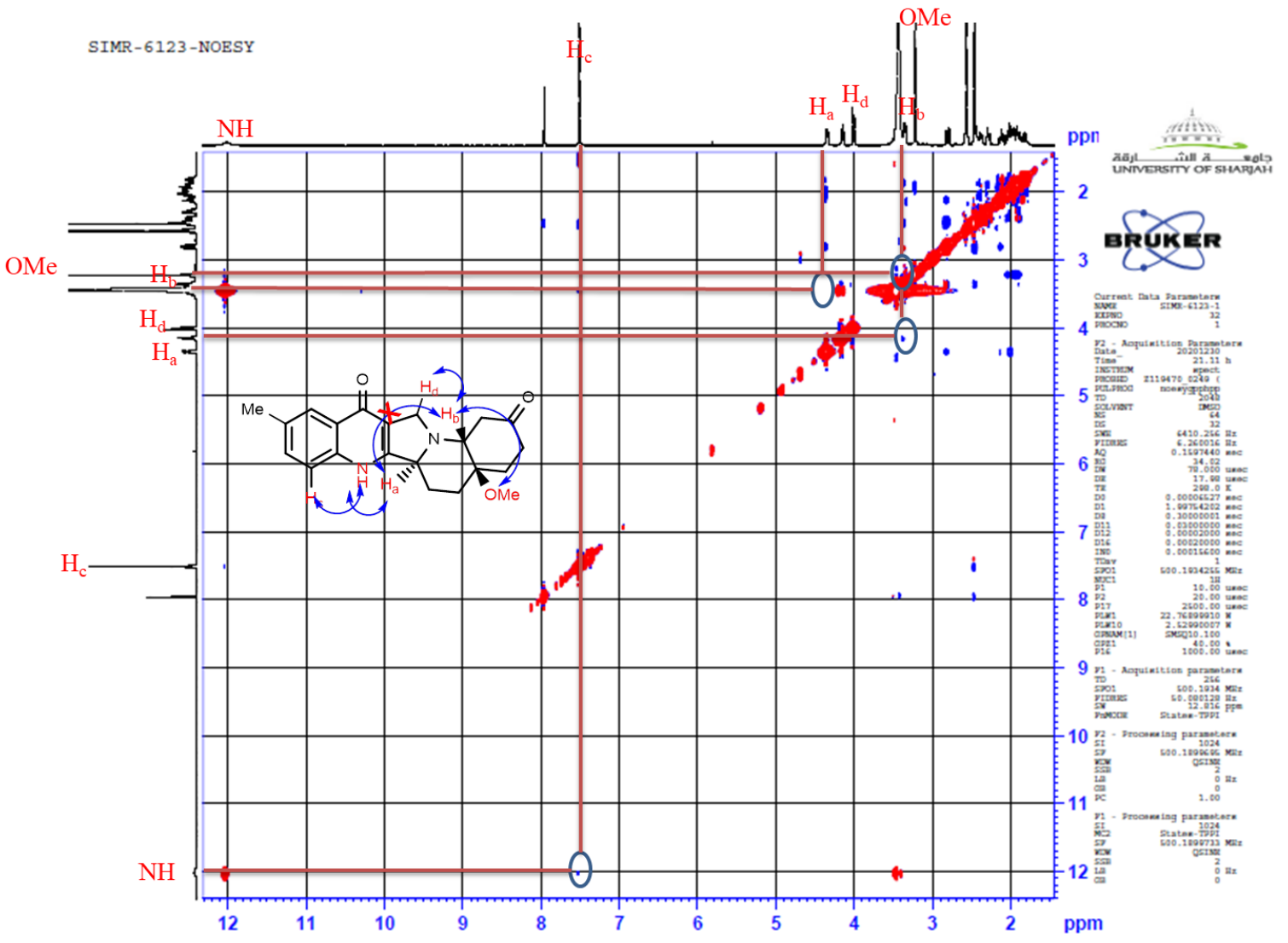

Figure S45 NOESY NMR (500 MHz, DMSO-d $\left.\mathrm{d}_{6}\right)$ spectrum of (4aR,6aS, 14aR)-4a-methoxy-10-methyl1,3,4,4a,5,6,6a, 7,13,14a-decahydropyrrolo[1,2-a:3,4-b']diquinoline-2,12-dione (6b) 


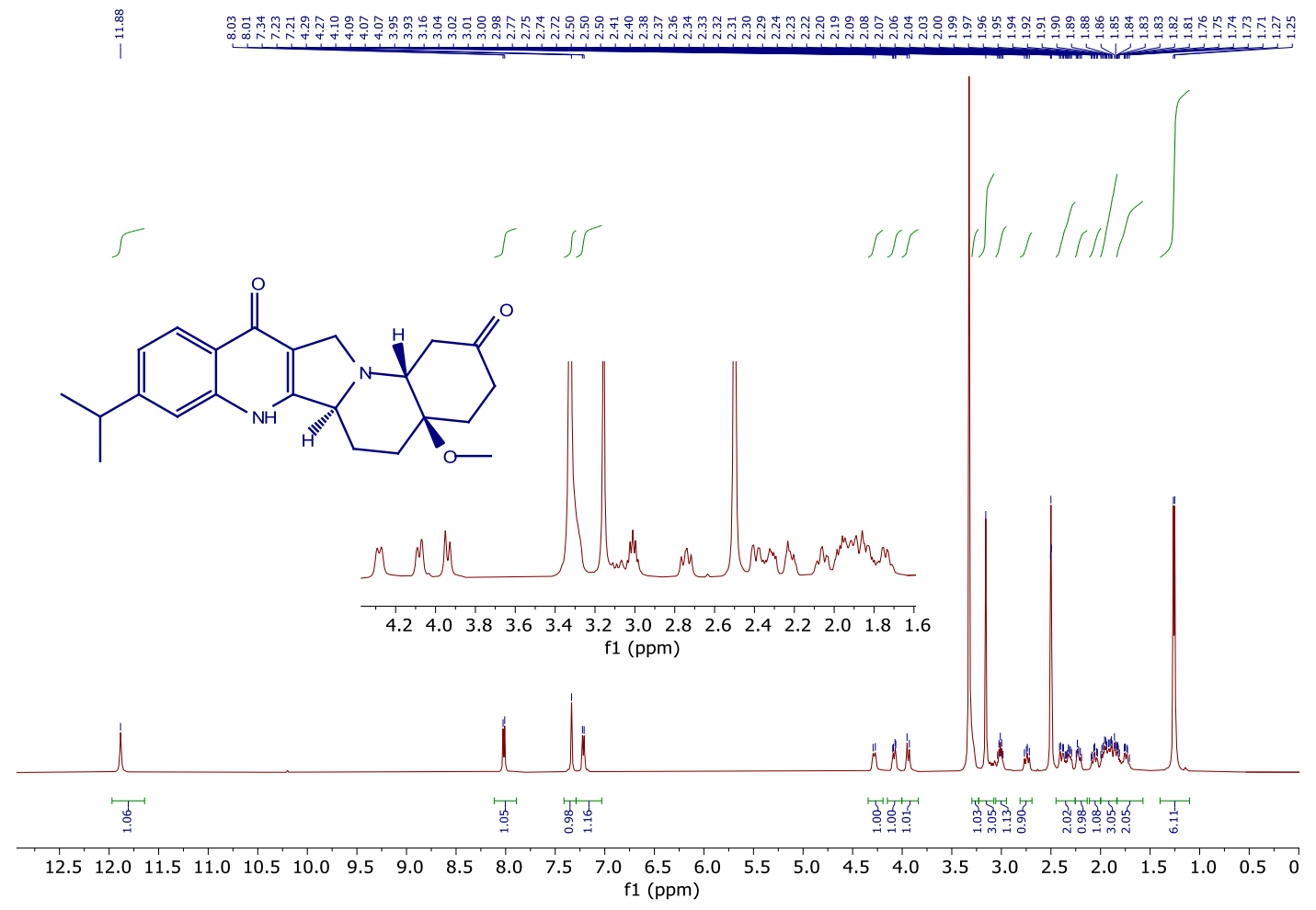

Figure S46 ${ }^{1} \mathrm{H}$ NMR (500 MHz, DMSO-d 6 ) spectrum of (4aR,6aS, 14aR)-9-isopropyl-4amethoxy-1,3,4,4a,5,6,6a,7,13,14a-decahydropyrrolo[1,2-a:3,4-b']diquinoline-2,12-dione (6c)

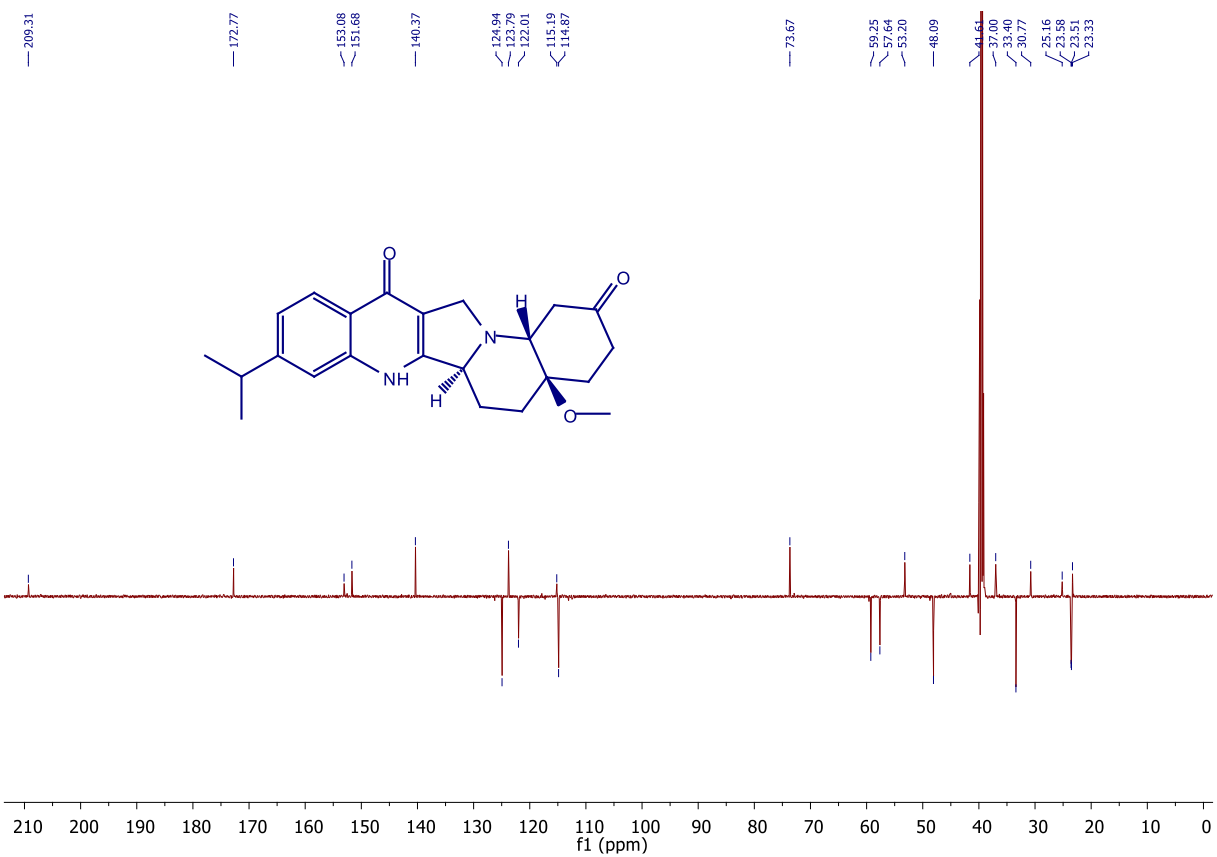

Figure $\mathrm{S47}{ }^{13} \mathrm{C}\left\{{ }^{1} \mathrm{H}\right\}$ APT NMR (125 MHz, DMSO-d $)$ spectrum of (4aR,6aS, 14aR)-9isopropyl-4a-methoxy-1,3,4,4a,5,6,6a,7,13,14a-decahydropyrrolo[1,2-a:3,4-b']diquinoline-2,12dione $(\mathbf{6 c})$ 


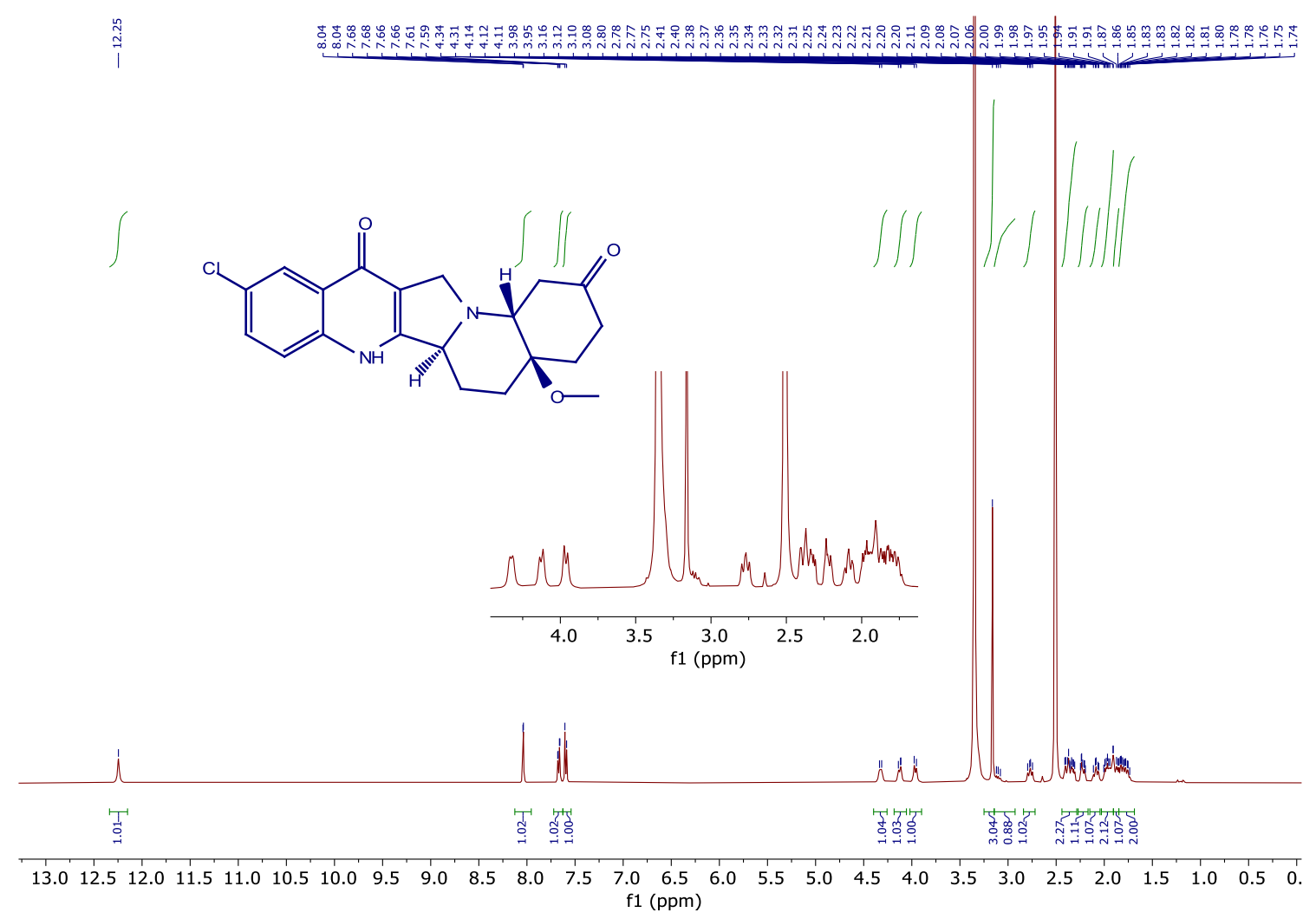

Figure $\mathbf{S 4 8}{ }^{1} \mathrm{H}$ NMR (500 MHz, DMSO-d 6 ) spectrum of (4aR,6aS, 14aR)-10-chloro-4amethoxy-1,3,4,4a,5,6,6a, 7,13,14a-decahydropyrrolo[1,2-a:3,4-b']diquinoline-2,12-dione (6d)

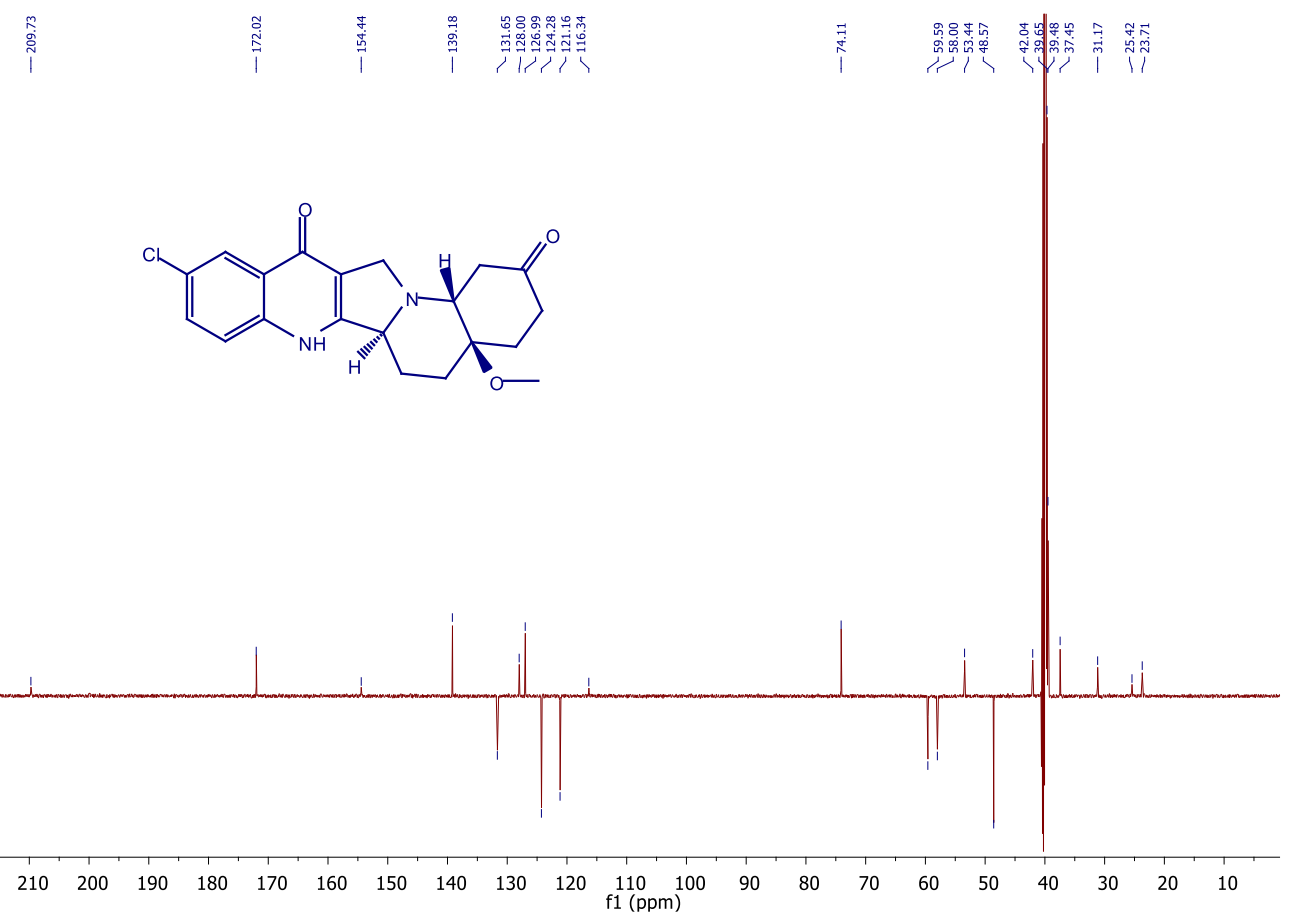

Figure S49 ${ }^{13} \mathrm{C}\left\{{ }^{1} \mathrm{H}\right\}$ APT NMR (125 MHz, DMSO-d 6 ) spectrum of (4aR,6aS, 14aR)-10-chloro4a-methoxy-1,3,4,4a,5,6,6a,7,13,14a-decahydropyrrolo[1,2-a:3,4-b']diquinoline-2,12-dione (6d) 


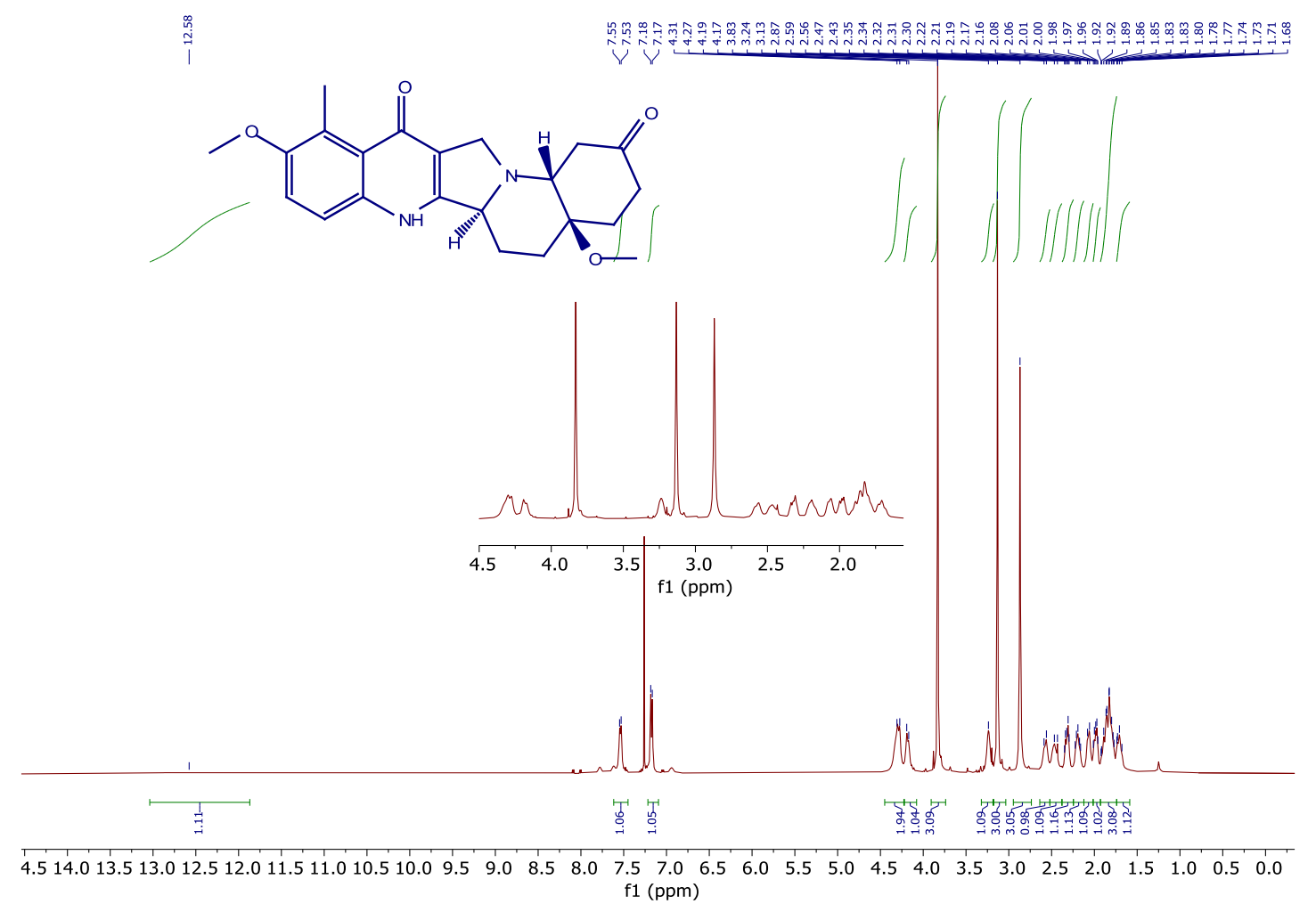

Figure S50 ${ }^{1} \mathrm{H}$ NMR $\left(500 \mathrm{MHz}, \mathrm{CDCl}_{3}\right)$ spectrum of (4aR,6aS,14aR)-4a,10-dimethoxy-11methyl-1,3,4,4a,5,6,6a, 7,13,14a-decahydropyrrolo[1,2-a:3,4-b']diquinoline-2,12-dione (6e)

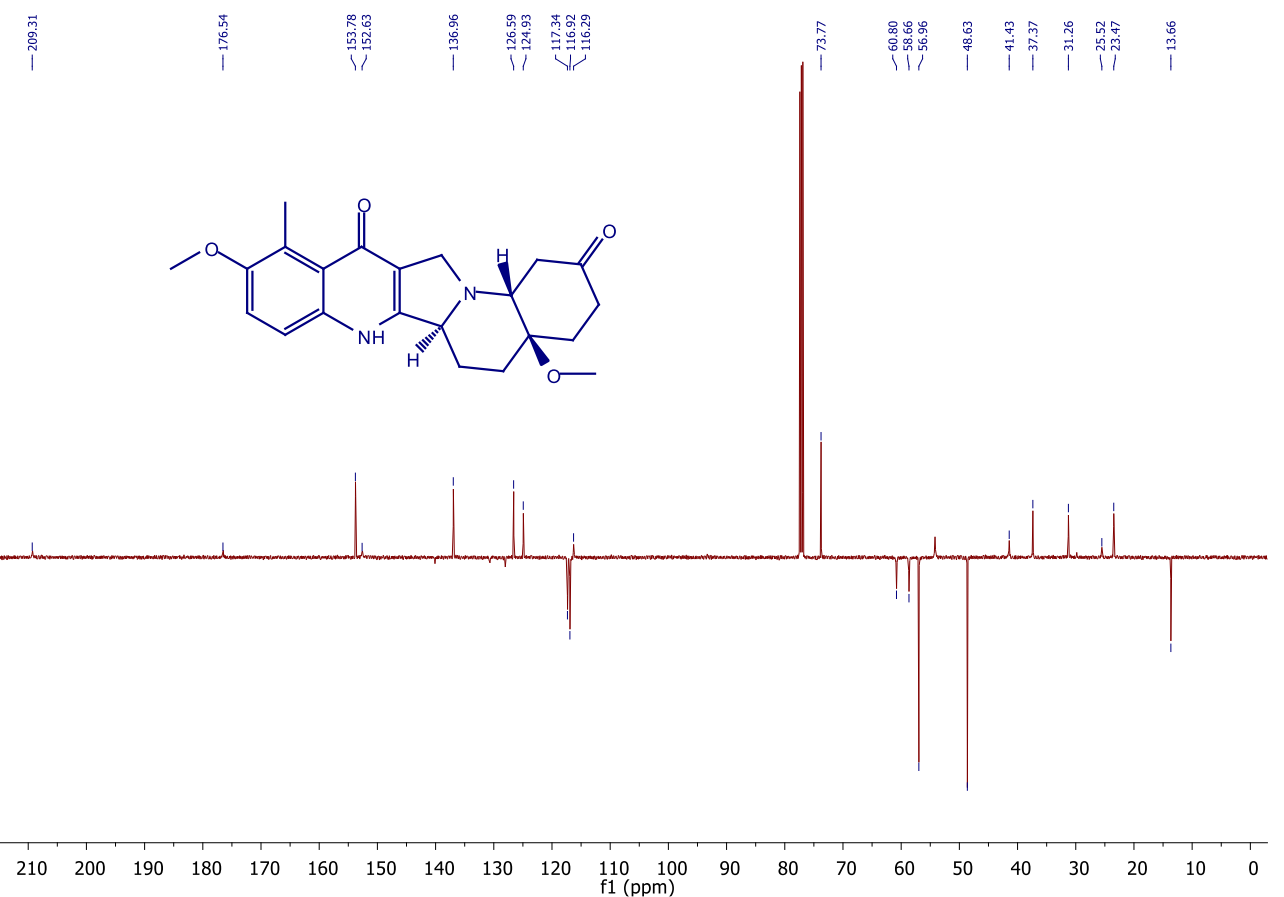

Figure S51 ${ }^{13} \mathrm{C}\left\{{ }^{1} \mathrm{H}\right\}$ APT NMR $\left(125 \mathrm{MHz}, \mathrm{CDCl}_{3}\right)$ spectrum of (4aR,6aS, 14aR)-4a, 10dimethoxy-11-methyl-1,3,4,4a,5,6,6a,7,13,14a-decahydropyrrolo[1,2-a:3,4-b']diquinoline-2,12dione $(\mathbf{6 e})$ 


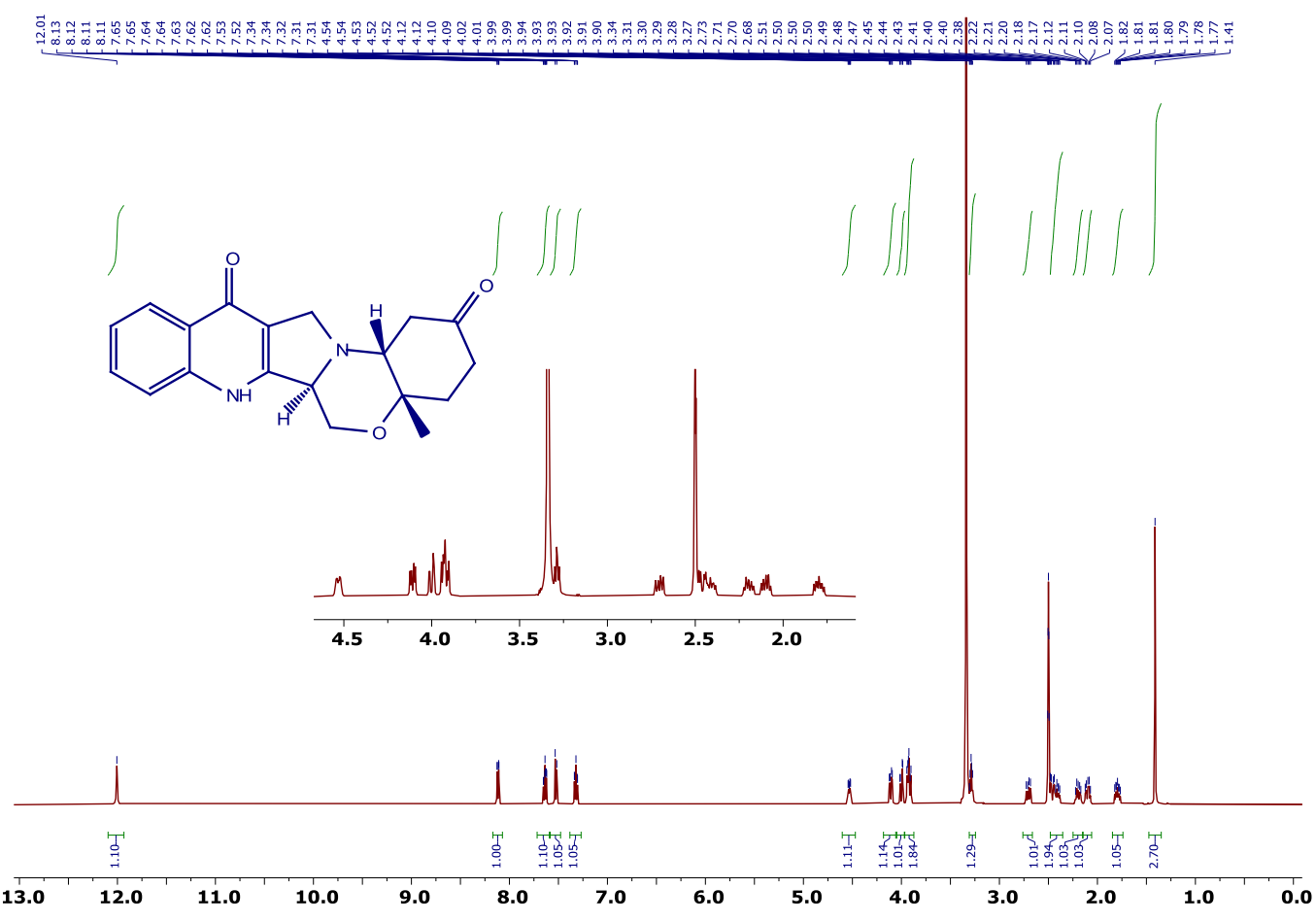

Figure S52 ${ }^{1} \mathrm{H}$ NMR (500 MHz, DMSO-d $)$ spectrum of (4aS,6aR, 14aR)-4a-methyl3,4,4a,6,6a,7,13,14a-octahydro-12H-benzo[5',6'][1,4]oxazino[4',3':1,2]pyrrolo[3,4-b]quinoline2,12(1H)-dione $(\mathbf{9})$
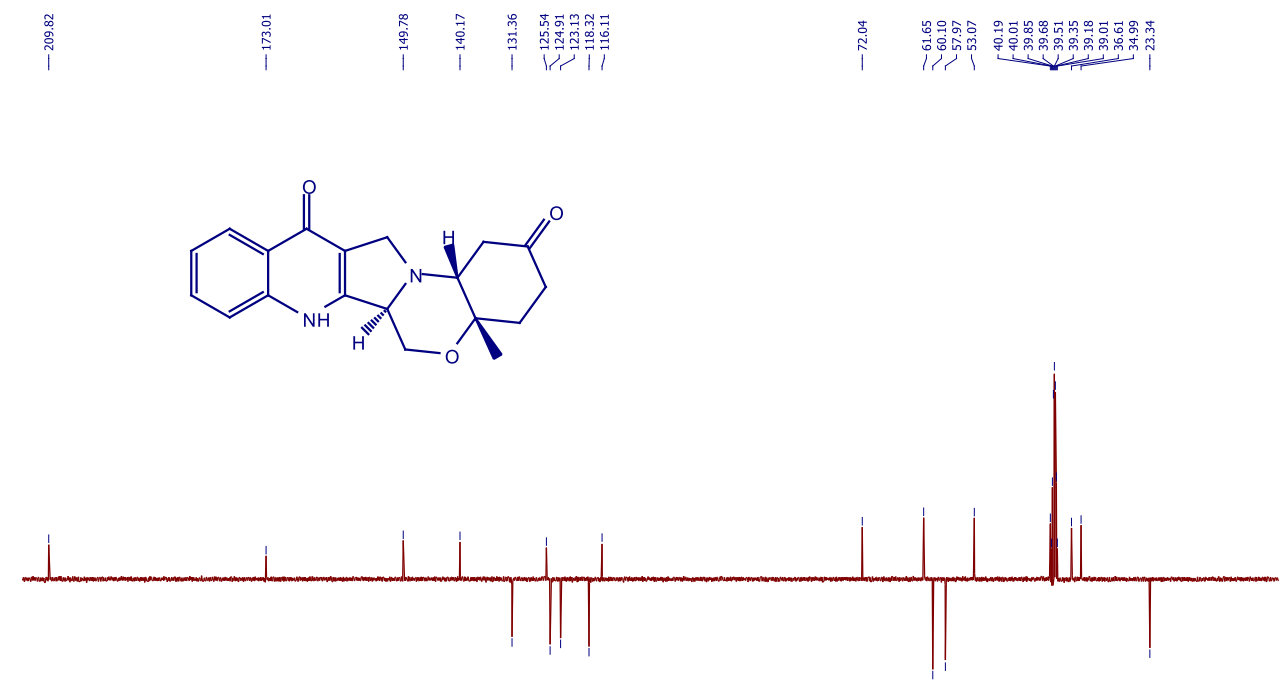

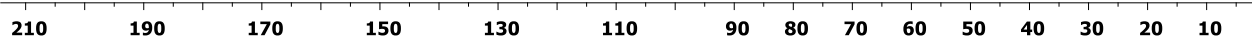

Figure S53 ${ }^{13} \mathrm{C}\left\{{ }^{1} \mathrm{H}\right\}$ APT NMR (125 MHz, DMSO-d 6 ) spectrum of (4aS,6aR,14aR)-4a-methyl3,4,4a,6,6a,7,13,14a-octahydro-12H-benzo[5',6'][1,4]oxazino[4',3':1,2]pyrrolo[3,4-b]quinoline-

2,12(1H)-dione $(\mathbf{9})$ 


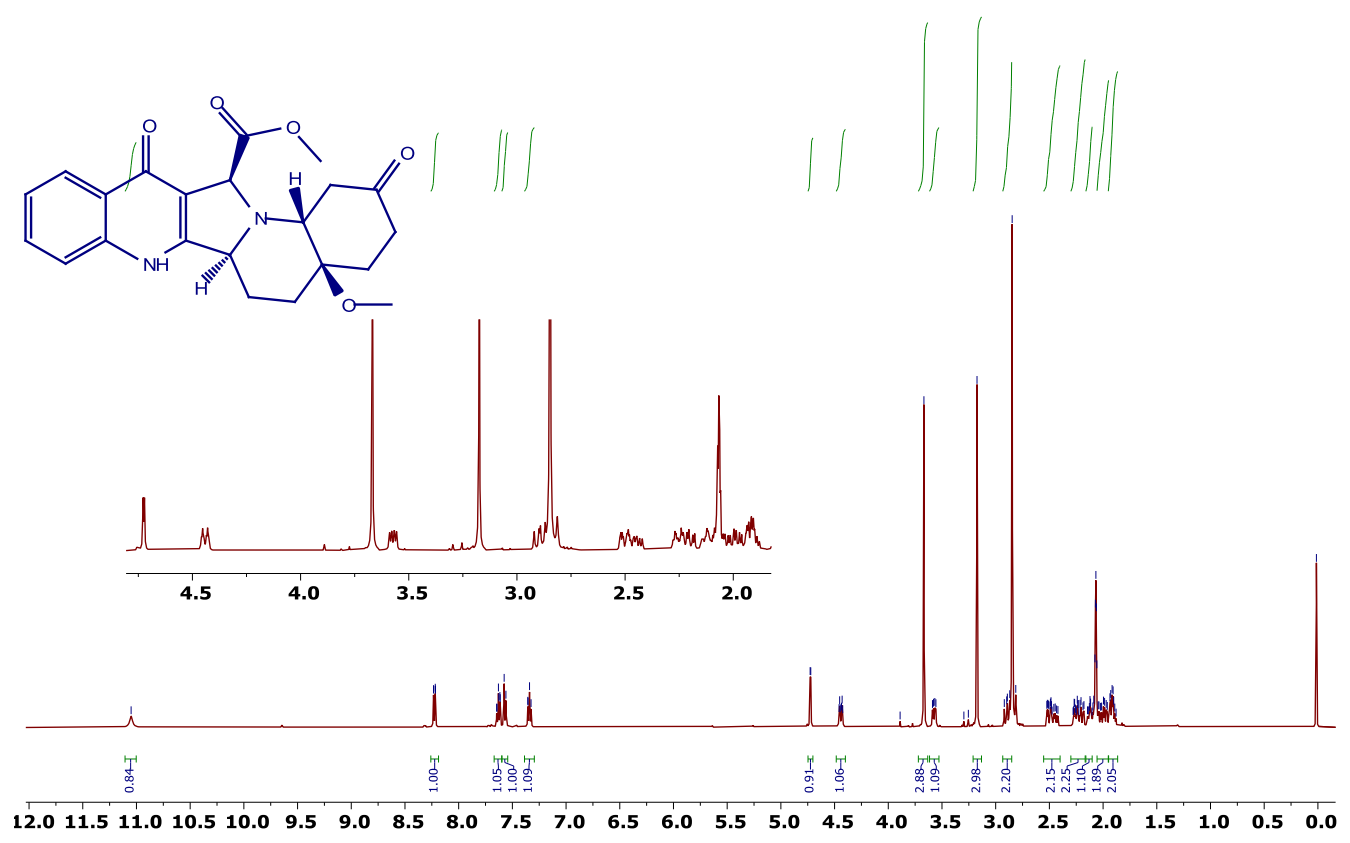

Figure S54 ${ }^{1} \mathrm{H}$ NMR (500 MHz, Acetone-d $)$ spectrum of methyl (4aR,6aS, 13S, 14aR)-4amethoxy-2,12-dioxo-1,2,3,4,4a,5,6,6a,7,12,13,14a-dodecahydropyrrolo[1,2-a:3,4-b']diquinoline13-carboxylate $(\mathbf{1 2 a})$

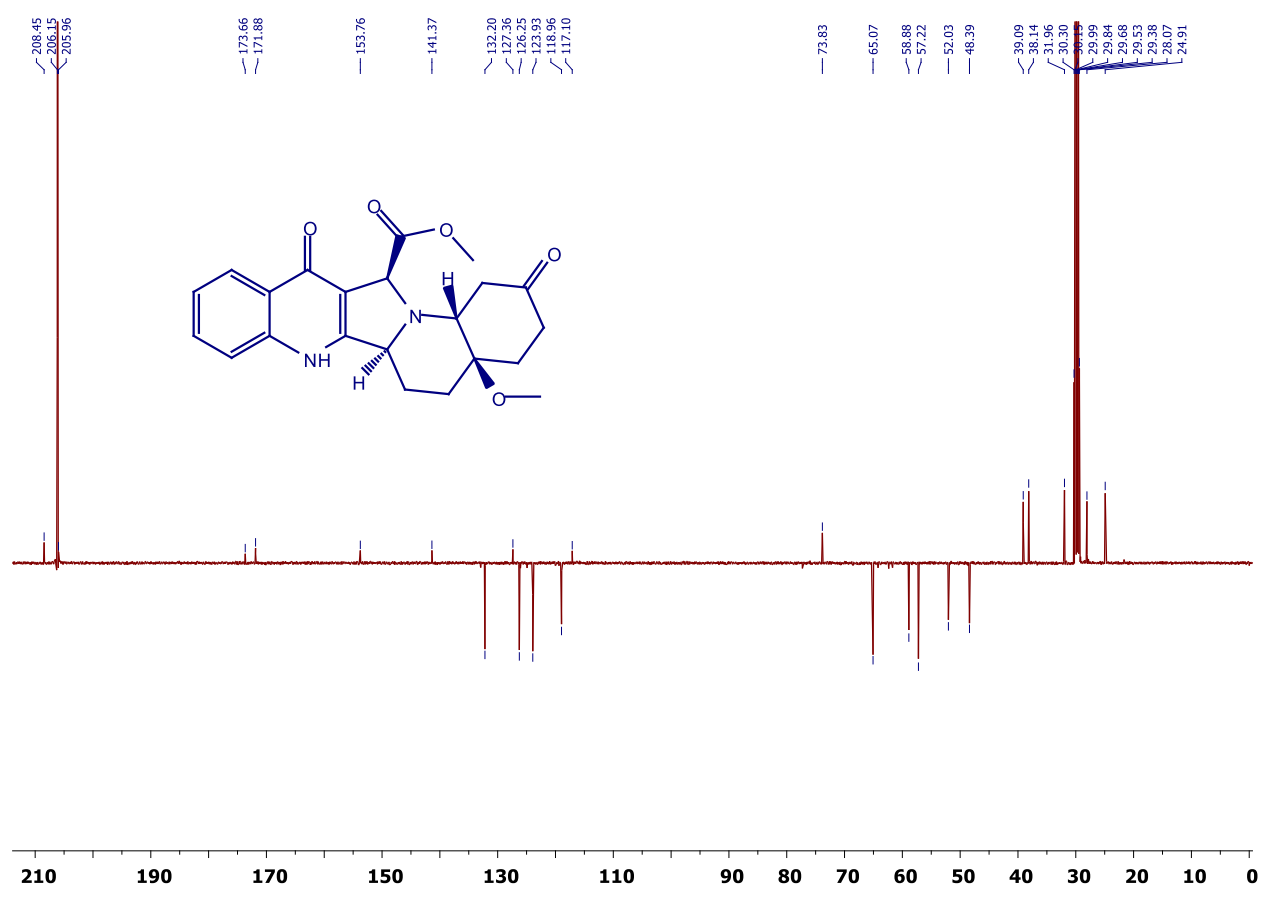

Figure S55 ${ }^{13} \mathrm{C}\left\{{ }^{1} \mathrm{H}\right\}$ APT NMR (125 MHz, Acetone-d $\left.\mathrm{d}_{6}\right)$ spectrum of methyl (4aR,6aS,13S,14aR)-4a-methoxy-2,12-dioxo-1,2,3,4,4a,5,6,6a,7,12,13,14adodecahydropyrrolo[1,2-a:3,4-b']diquinoline-13-carboxylate (12a) 


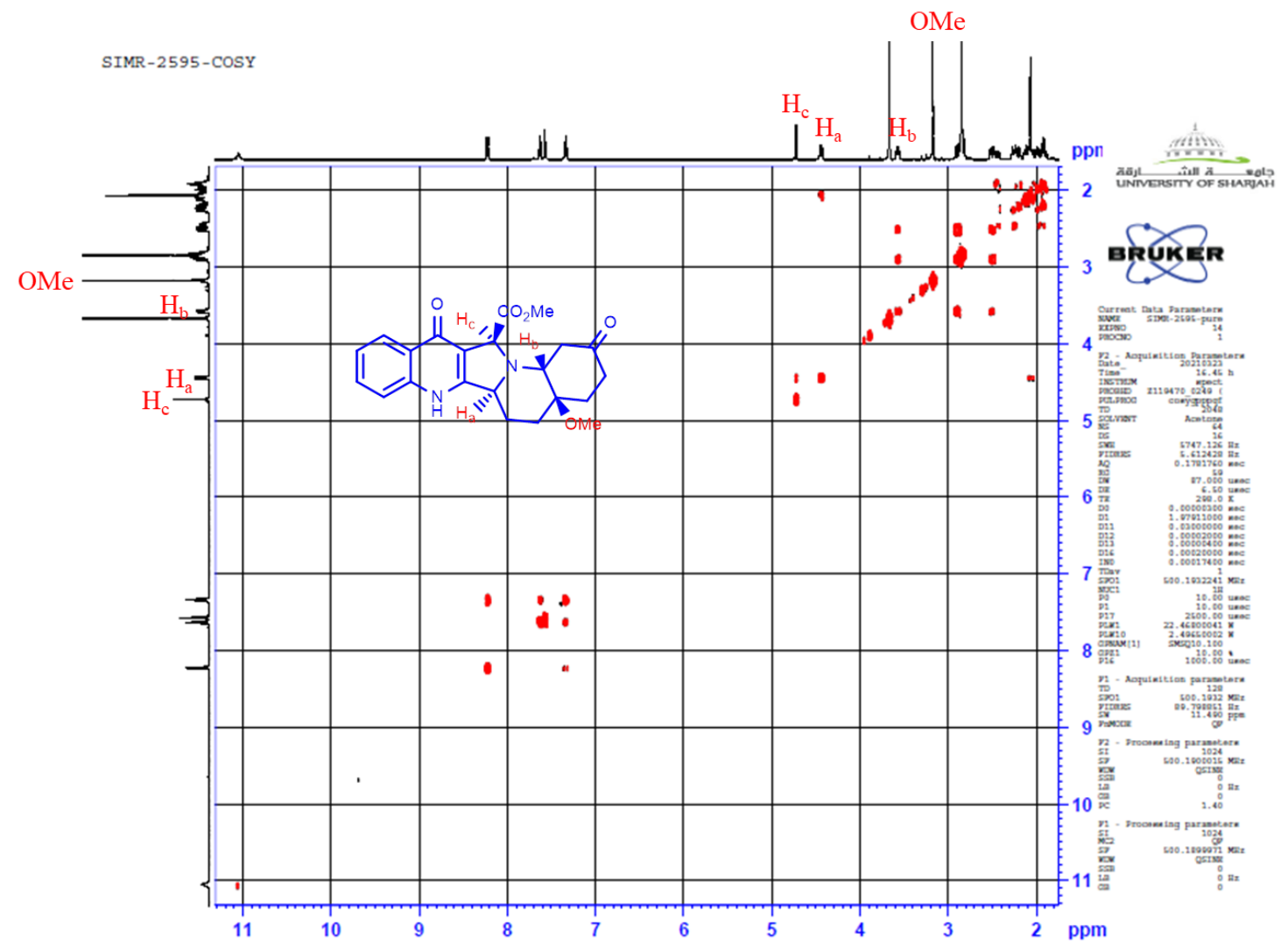

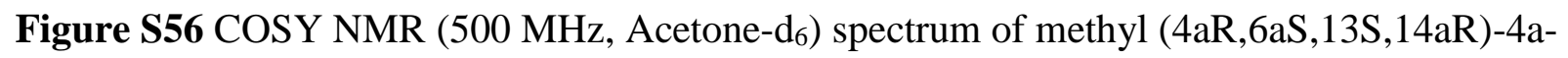
methoxy-2,12-dioxo-1,2,3,4,4a,5,6,6a,7,12,13,14a-dodecahydropyrrolo[1,2-a:3,4-b']diquinoline13-carboxylate (12a) 


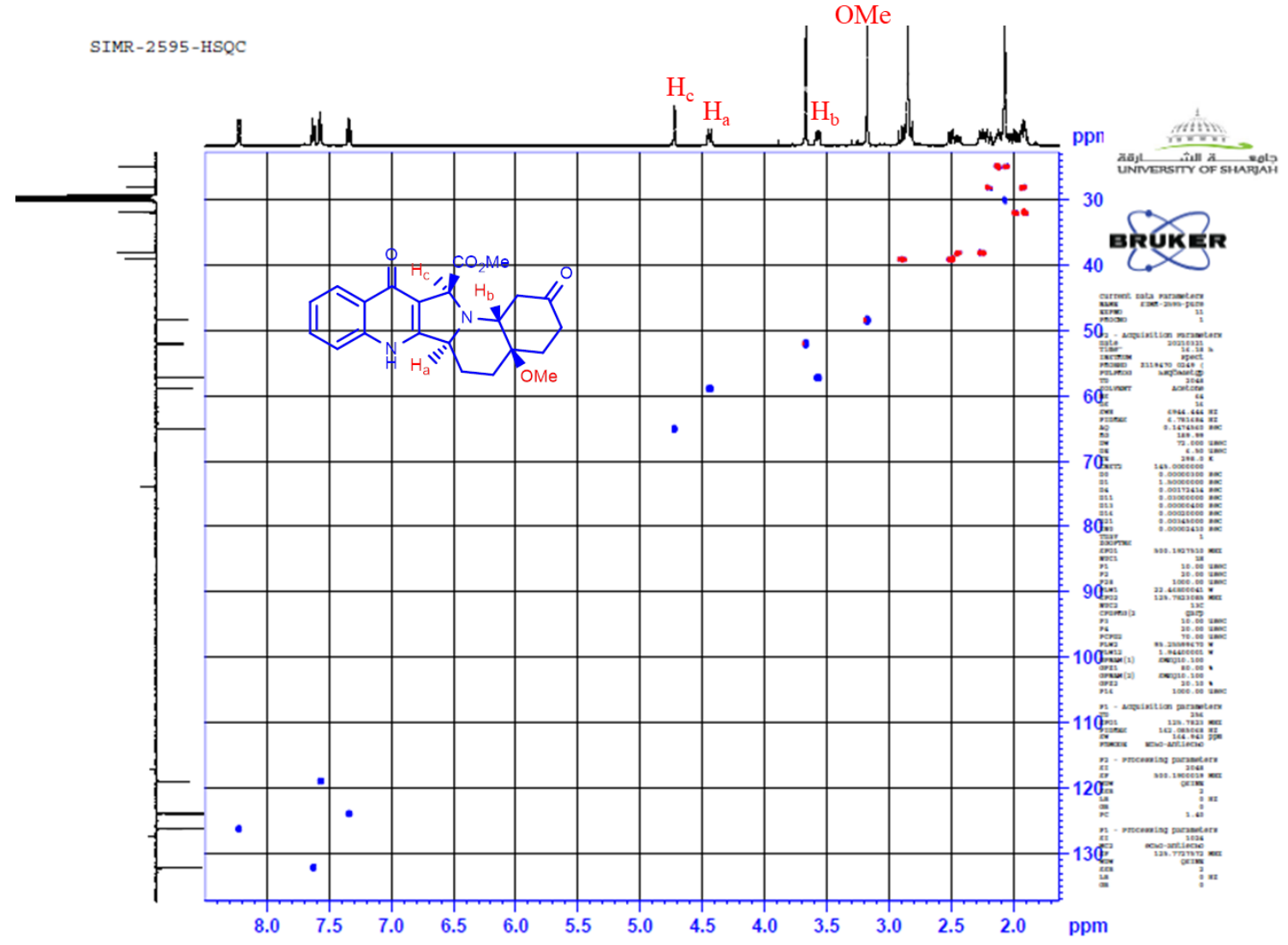

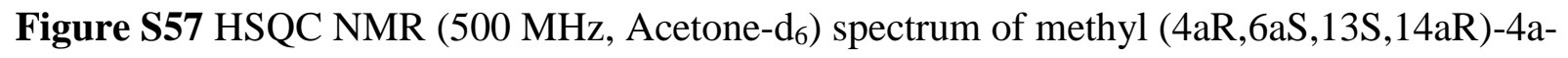
methoxy-2,12-dioxo-1,2,3,4,4a,5,6,6a,7,12,13,14a-dodecahydropyrrolo[1,2-a:3,4-b']diquinoline13-carboxylate (12a) 


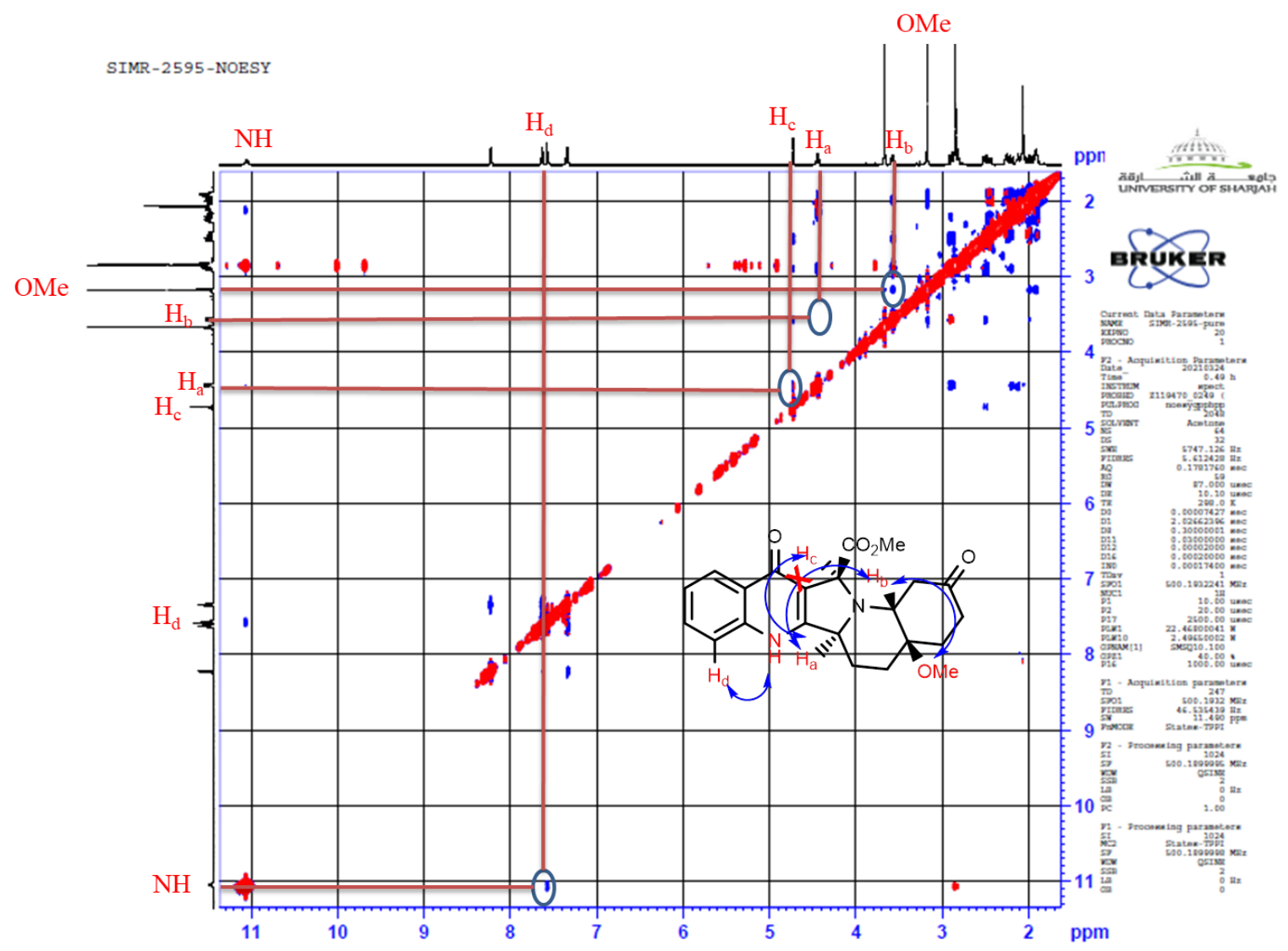

Figure S58 NOESY NMR (500 MHz, Acetone-d ${ }_{6}$ ) spectrum of methyl (4aR,6aS, 13S,14aR)-4amethoxy-2,12-dioxo-1,2,3,4,4a,5,6,6a,7,12,13,14a-dodecahydropyrrolo[1,2-a:3,4-b']diquinoline13-carboxylate (12a) 


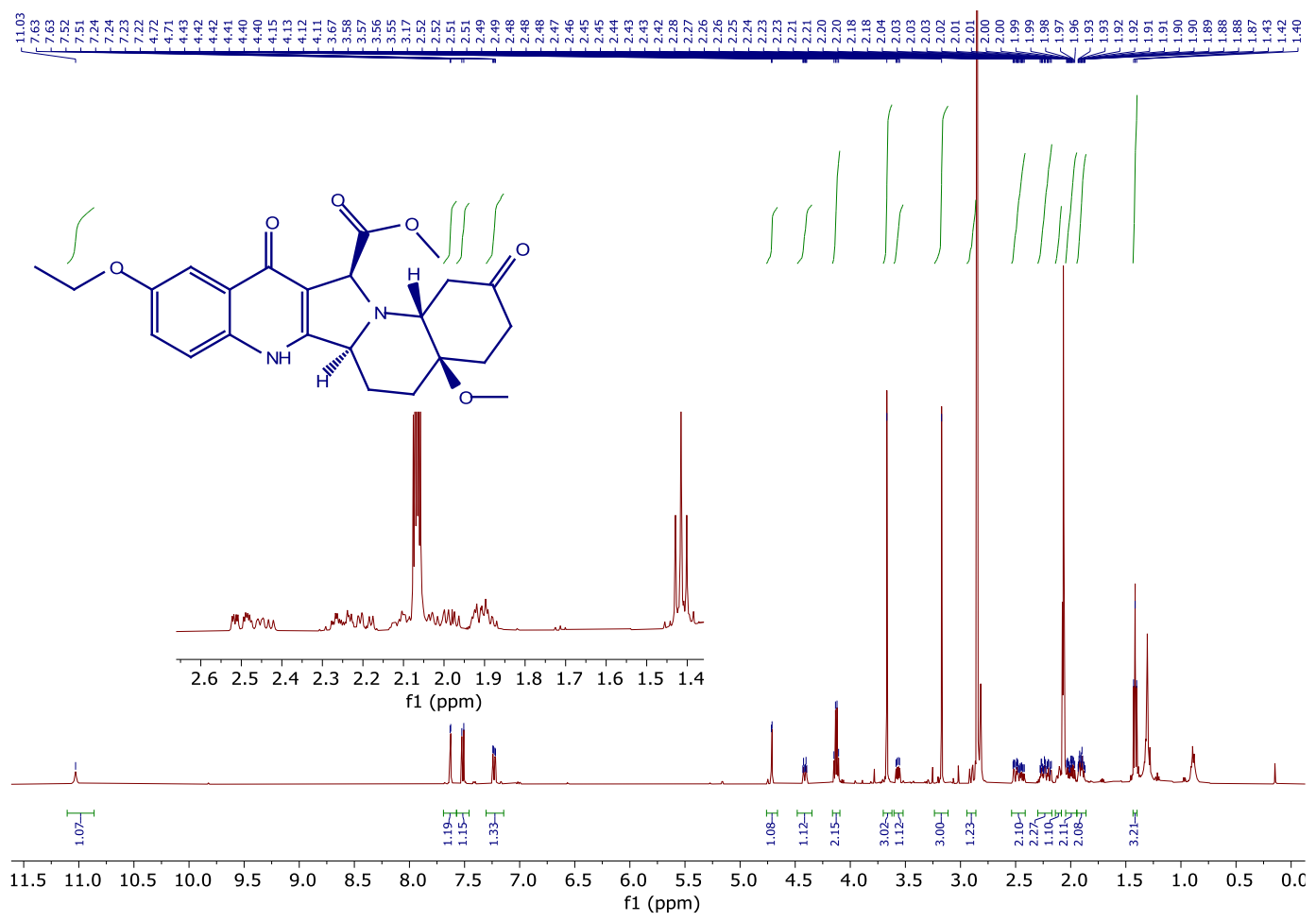

Figure S59 ${ }^{1} \mathrm{H}$ NMR (500 MHz, Acetone-d $\left.{ }_{6}\right)$ spectrum of methyl (4aR,6aS,13S,14aR)-10ethoxy-4a-methoxy-2,12-dioxo-1,2,3,4,4a,5,6,6a,7,12,13,14a-dodecahydropyrrolo[1,2-a:3,4$\mathrm{b}^{\prime}$ ]diquinoline-13-carboxylate $(\mathbf{1 2 b})$

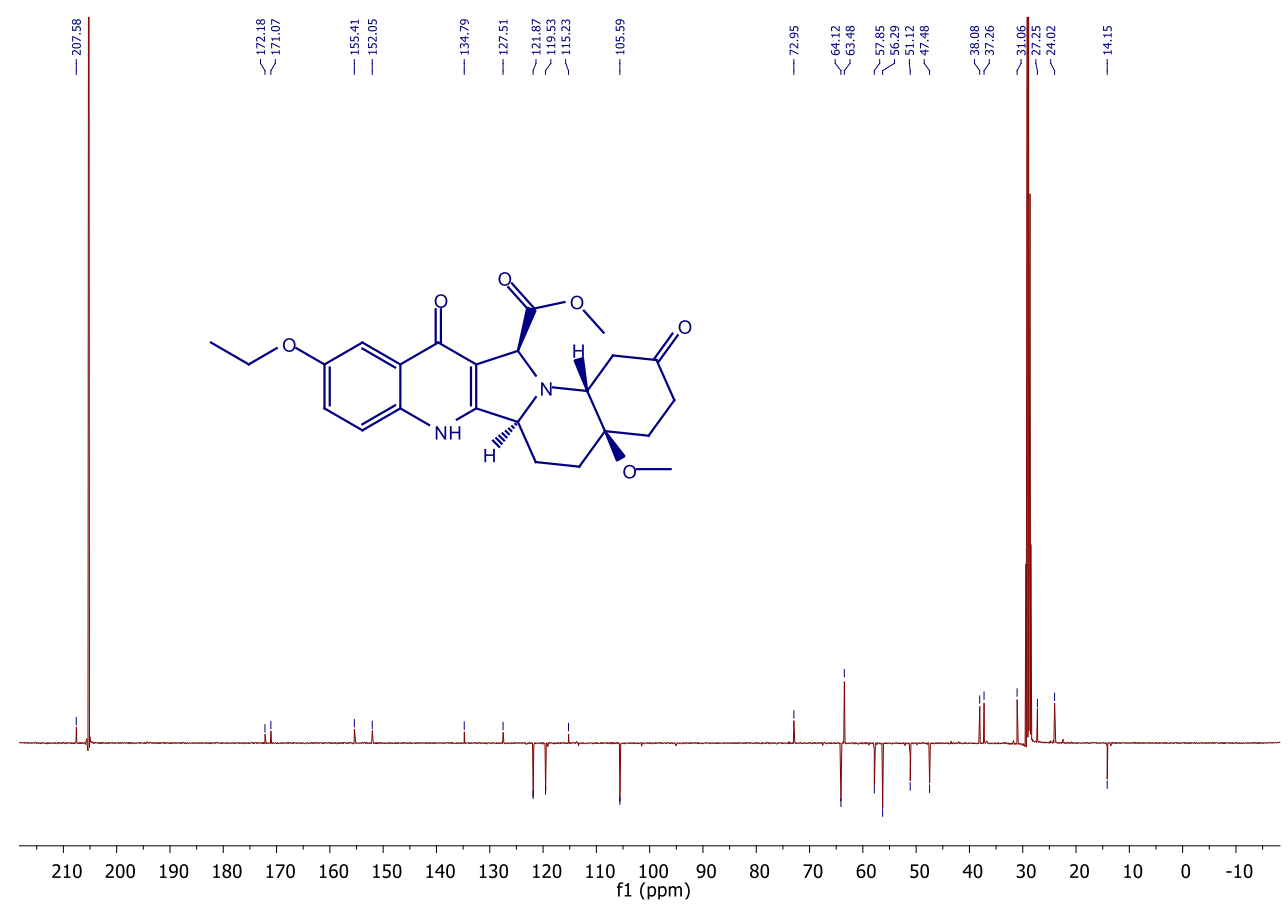

Figure S60 ${ }^{13} \mathrm{C}\left\{{ }^{1} \mathrm{H}\right\}$ APT NMR (125 MHz, Acetone-d 6 ) spectrum of methyl (4aR,6aS, 13S, 14aR)-10-ethoxy-4a-methoxy-2,12-dioxo-1,2,3,4,4a, 5,6,6a, 7,12,13,14adodecahydropyrrolo[1,2-a:3,4-b']diquinoline-13-carboxylate (12b) 

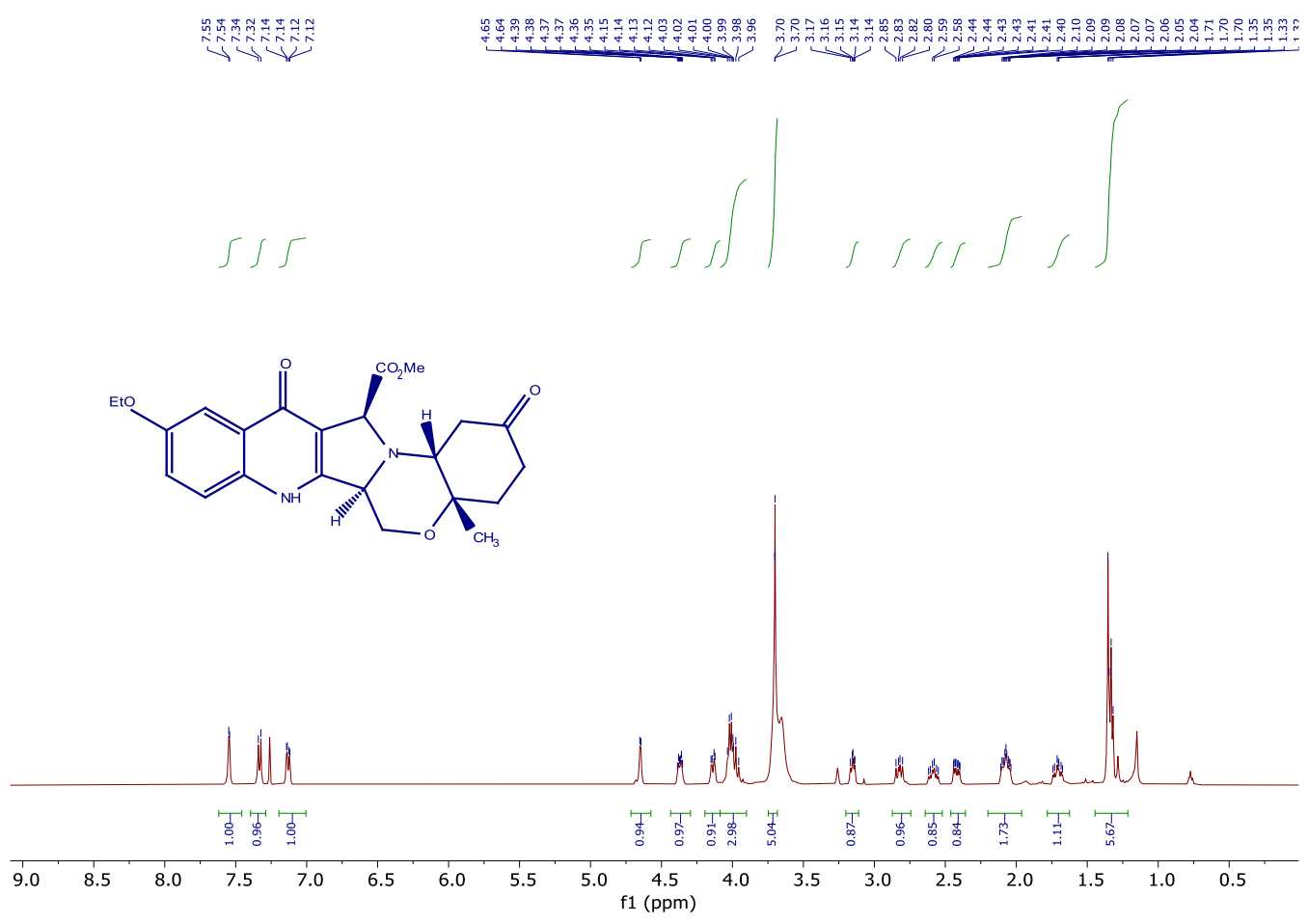

Figure S61 ${ }^{1} \mathrm{H}$ NMR (500 MHz, $\left.\mathrm{CDCl}_{3}\right)$ spectrum of methyl (4aS,6aR,13S,14aR)-10-ethoxy-4amethoxy-2,12-dioxo-1,2,3,4,4a,6a,7,12,13,14a-decahydro-6Hbenzo[5',6'][1,4]oxazino[4',3':1,2]pyrrolo[3,4-b]quinoline-13-carboxylate (15)

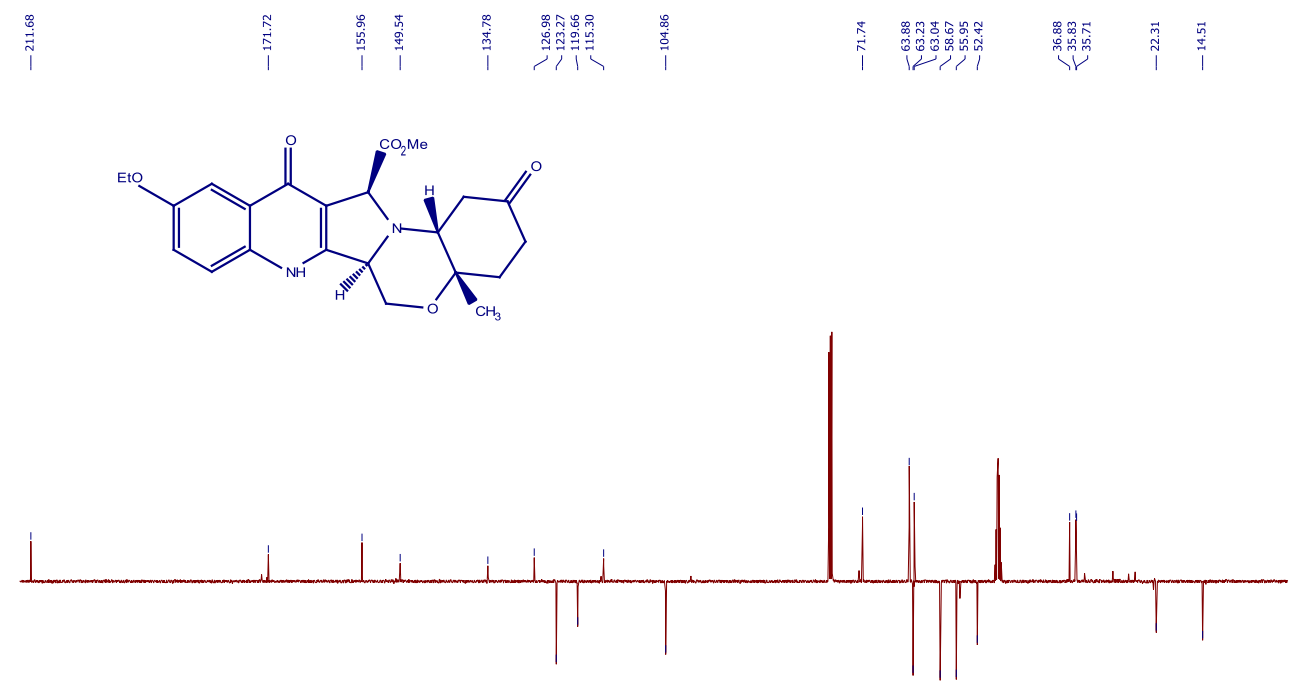

$\begin{array}{llllllllllllllllllllllllllll}210 & 200 & 190 & 180 & 170 & 160 & 150 & 140 & 130 & 120 & 110 & 100 & 90 & 80 & 70 & 60 & 50 & 40 & 30 & 20 & 10 & \end{array}$

Figure S62 ${ }^{13} \mathrm{C}\left\{{ }^{1} \mathrm{H}\right\}$ APT NMR (125 MHz, $\left.\mathrm{CDCl}_{3}\right)$ spectrum of methyl (4aS,6aR,13S,14aR)-10ethoxy-4a-methoxy-2,12-dioxo-1,2,3,4,4a,6a,7,12,13,14a-decahydro-6Hbenzo[5',6'][1,4]oxazino[4',3':1,2]pyrrolo[3,4-b]quinoline-13-carboxylate (15) 


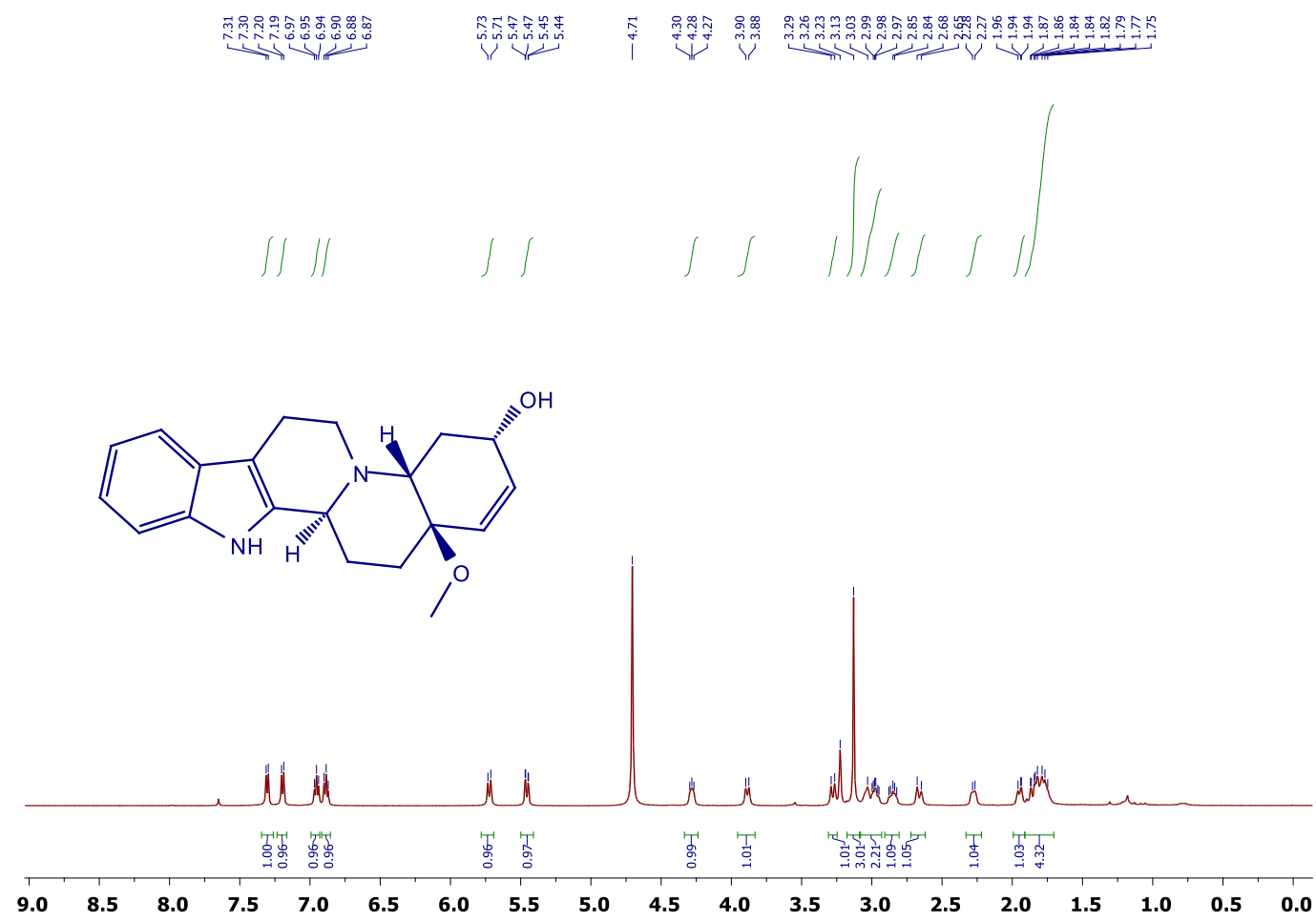

Figure S63 ${ }^{1} \mathrm{H}\left(500 \mathrm{MHz}, \mathrm{CD}_{3} \mathrm{OD}\right)$ spectrum of (2aR,5S,6aR,14bS)-2a-methoxy1,2,2a,5,6,6a,8,9,14,14b-decahydroindolo[2',3':3,4]pyrido[1,2-a]quinolin-5-ol (16)

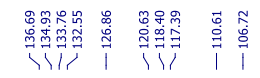

$\sqrt{\text { inj }}$
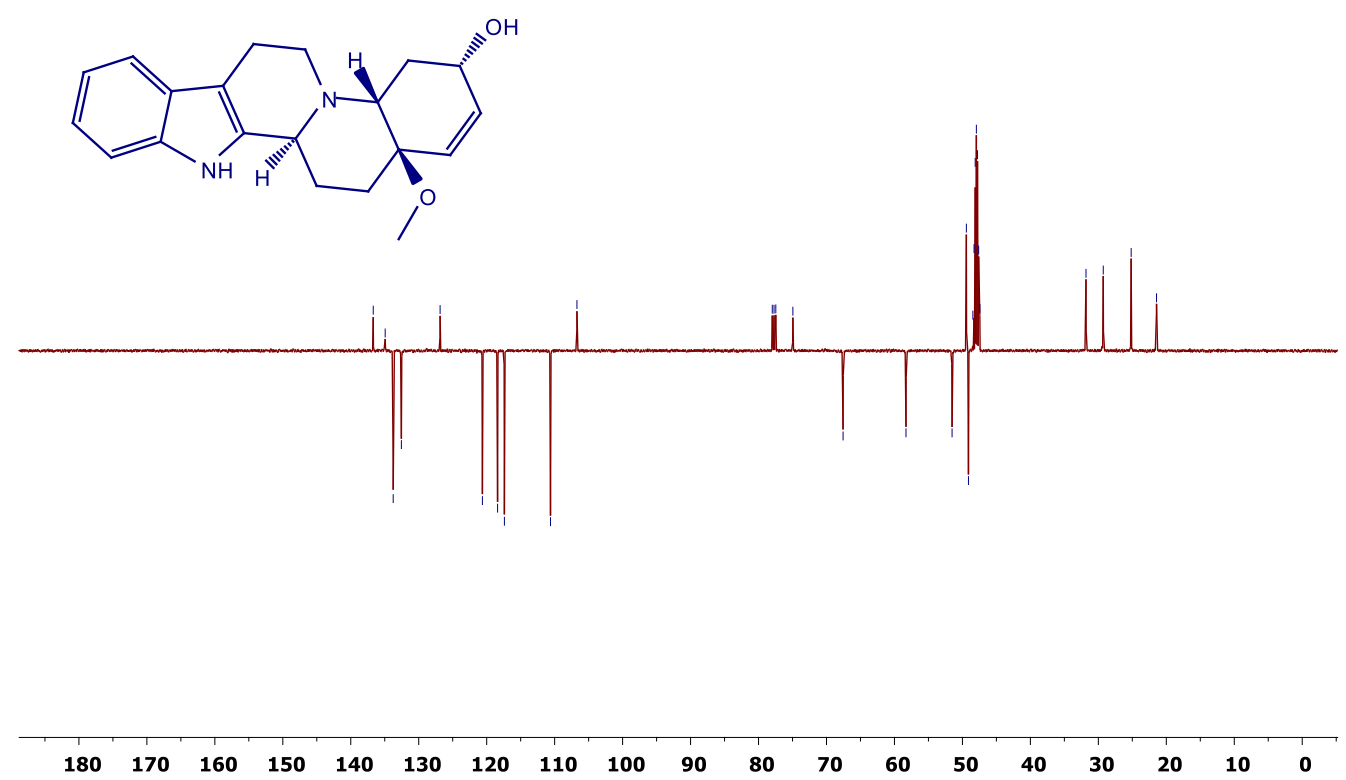

Figure S64 ${ }^{13} \mathrm{C}\left\{{ }^{1} \mathrm{H}\right\}$ APT NMR (125 MHz, CD 3 OD) spectrum of (2aR,5S,6aR,14bS)-2a-methoxy1,2,2a,5,6,6a,8,9,14,14b-decahydroindolo[2',3':3,4]pyrido[1,2-a]quinolin-5-ol (16) 


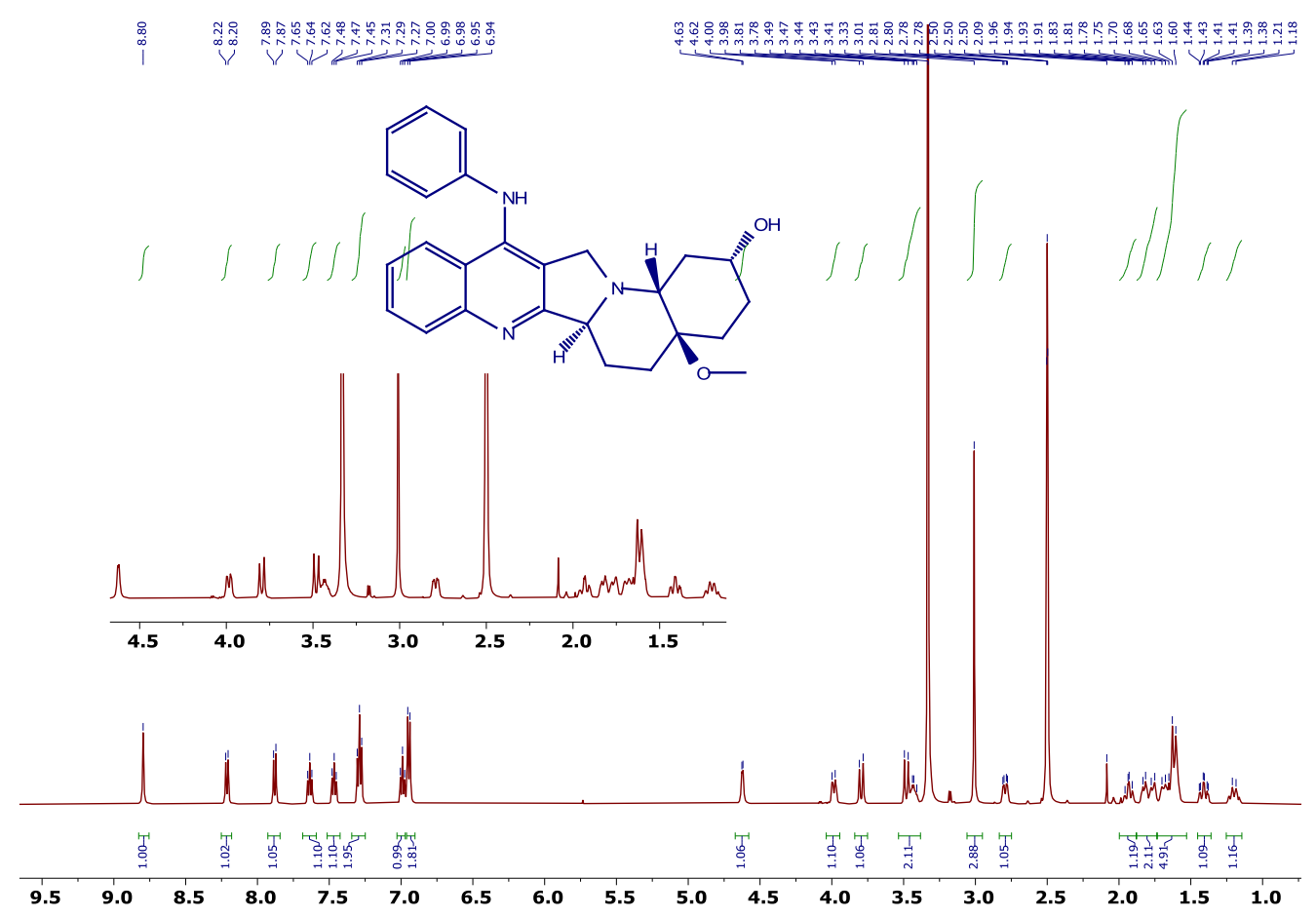

Figure S65 ${ }^{1} \mathrm{H}$ NMR (500 MHz, DMSO-d $)$ spectrum of (2R,4aR,6aS,14aR)-4a-methoxy-12(phenylamino)-1,2,3,4,4a,5,6,6a,13,14a-decahydropyrrolo[1,2-a:3,4-b']diquinolin-2-ol (19a)
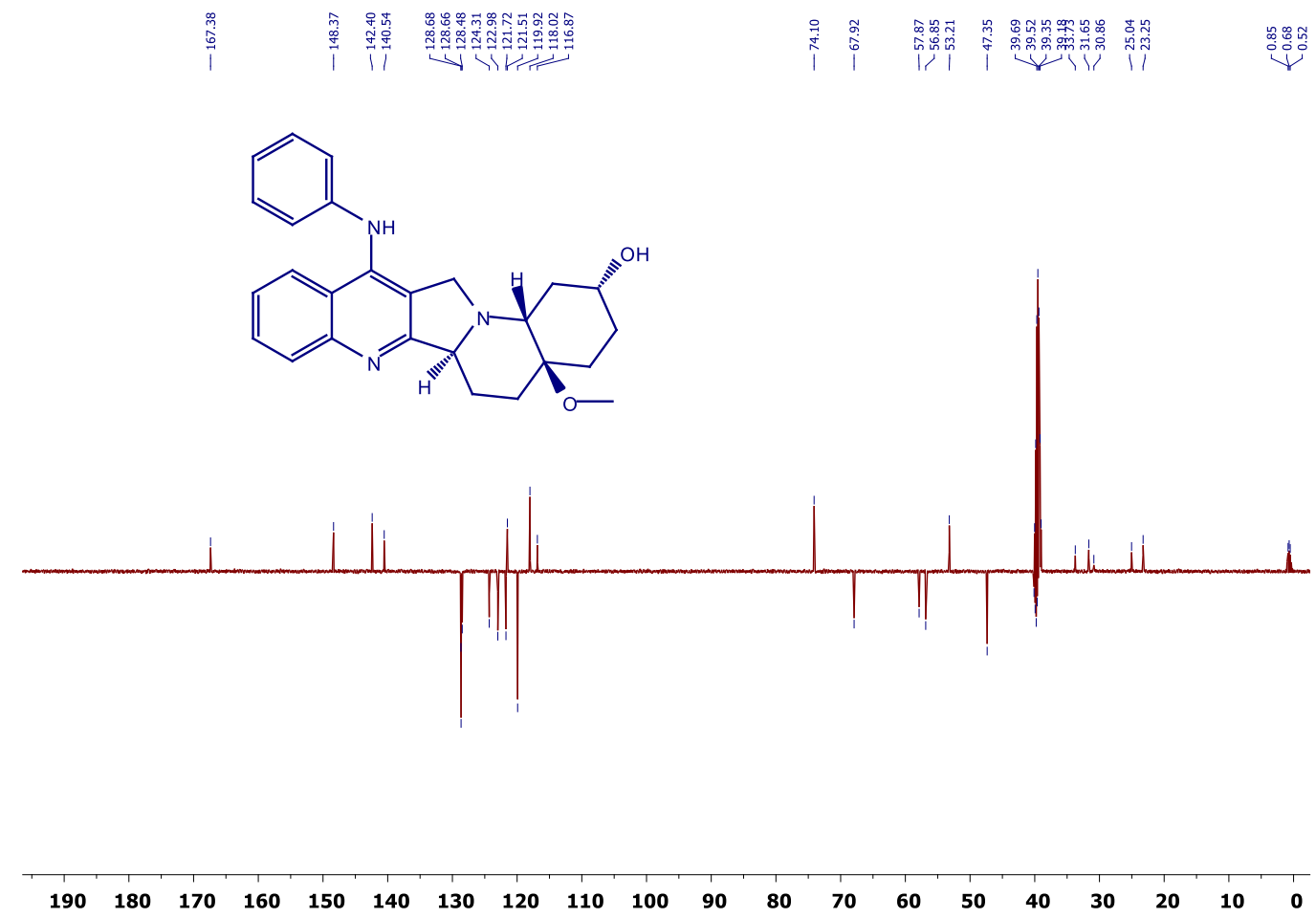

Figure S66 ${ }^{13} \mathrm{C}\left\{{ }^{1} \mathrm{H}\right\}$ APT NMR (125 MHz, DMSO-d 6 ) spectrum of $(2 \mathrm{R}, 4 \mathrm{aR}, 6 \mathrm{aS}, 14 \mathrm{aR})-4 \mathrm{a}-$ methoxy-12-(phenylamino)-1,2,3,4,4a,5,6,6a,13,14a-decahydropyrrolo[1,2-a:3,4-b']diquinolin-2ol (19a) 


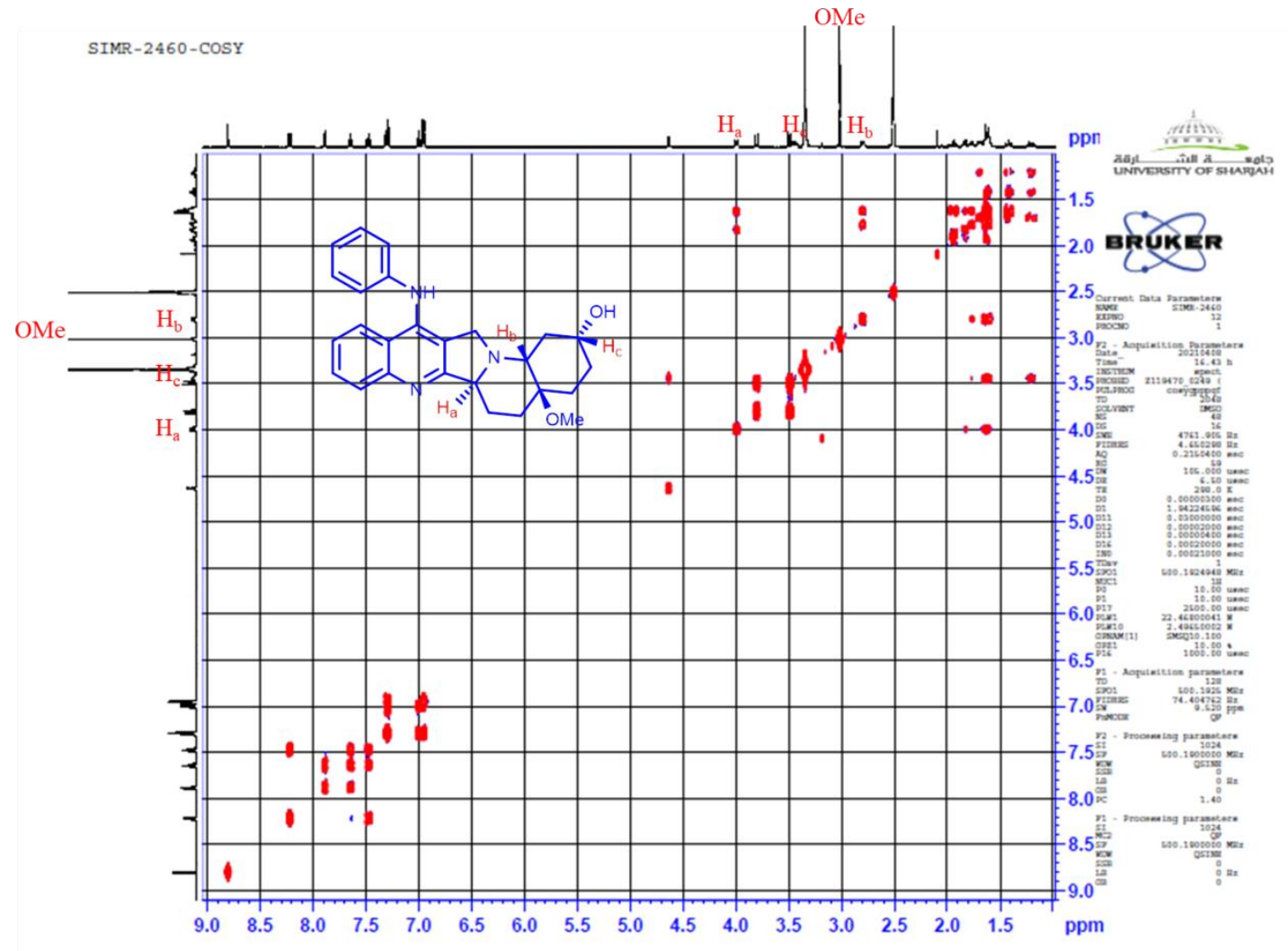

Figure S67 COSY NMR (500 MHz, DMSO-d $)$ spectrum of (2R,4aR,6aS, 14aR)-4a-methoxy12-(phenylamino)-1,2,3,4,4a,5,6,6a,13,14a-decahydropyrrolo[1,2-a:3,4-b']diquinolin-2-ol (19a) 


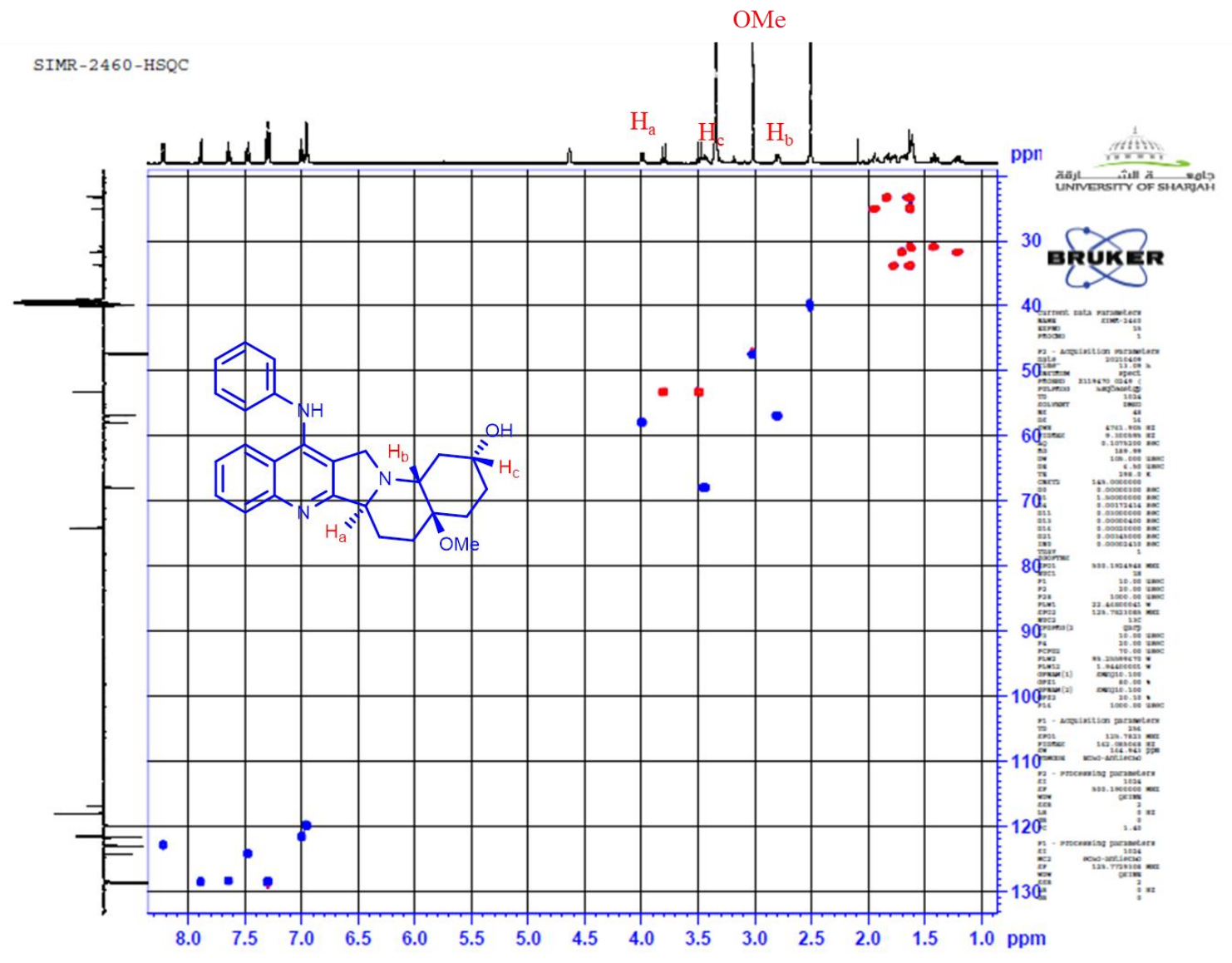

Figure S68 HSQC NMR (500 MHz, DMSO-d $)$ spectrum of (2R,4aR,6aS, 14aR)-4a-methoxy12-(phenylamino)-1,2,3,4,4a,5,6,6a,13,14a-decahydropyrrolo[1,2-a:3,4-b']diquinolin-2-ol (19a) 


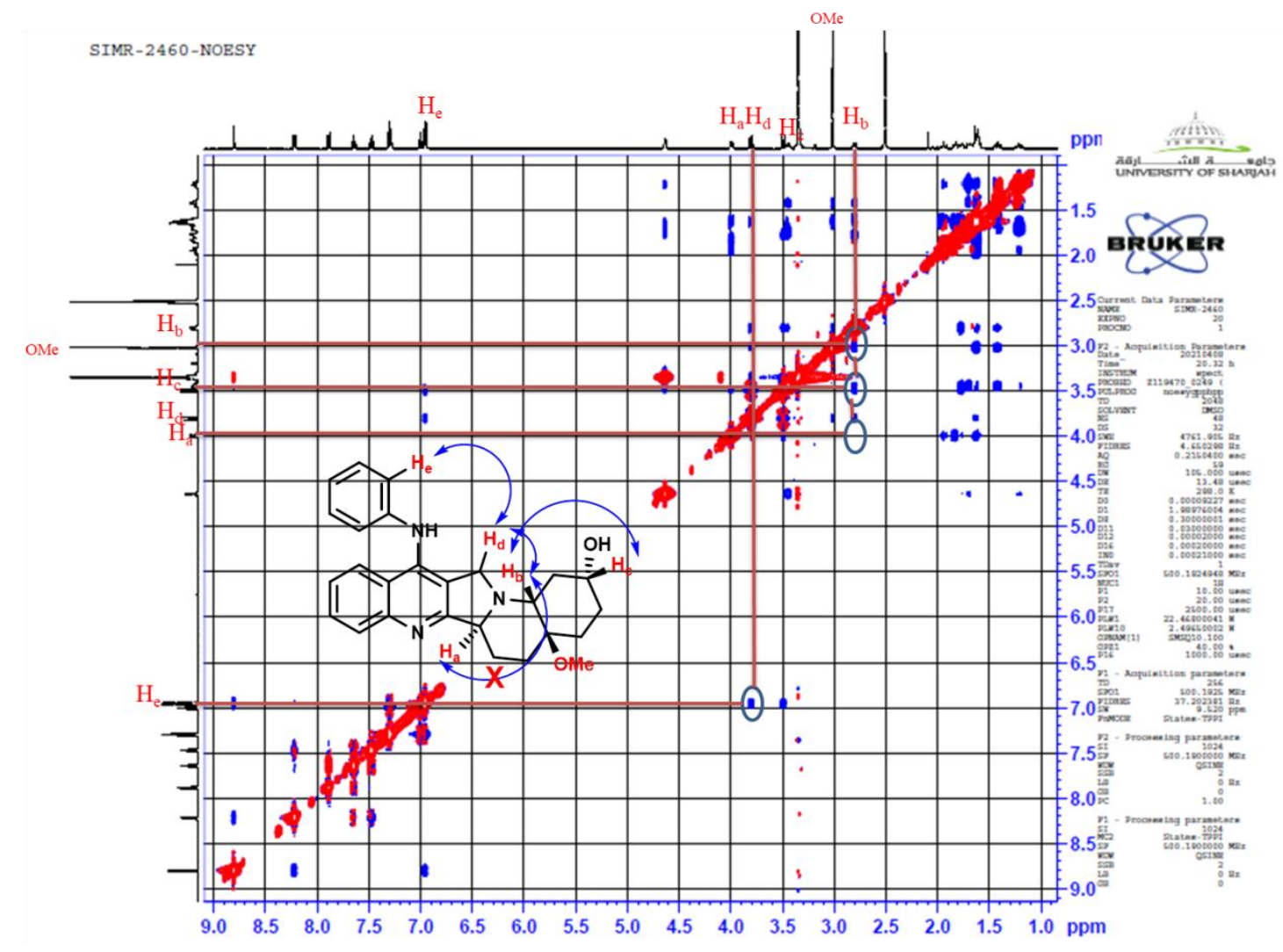

Figure S69 NOESY NMR (500 MHz, DMSO-d 6 ) spectrum of (2R,4aR,6aS, 14aR)-4a-methoxy12-(phenylamino)-1,2,3,4,4a,5,6,6a,13,14a-decahydropyrrolo[1,2-a:3,4-b']diquinolin-2-ol (19a) 


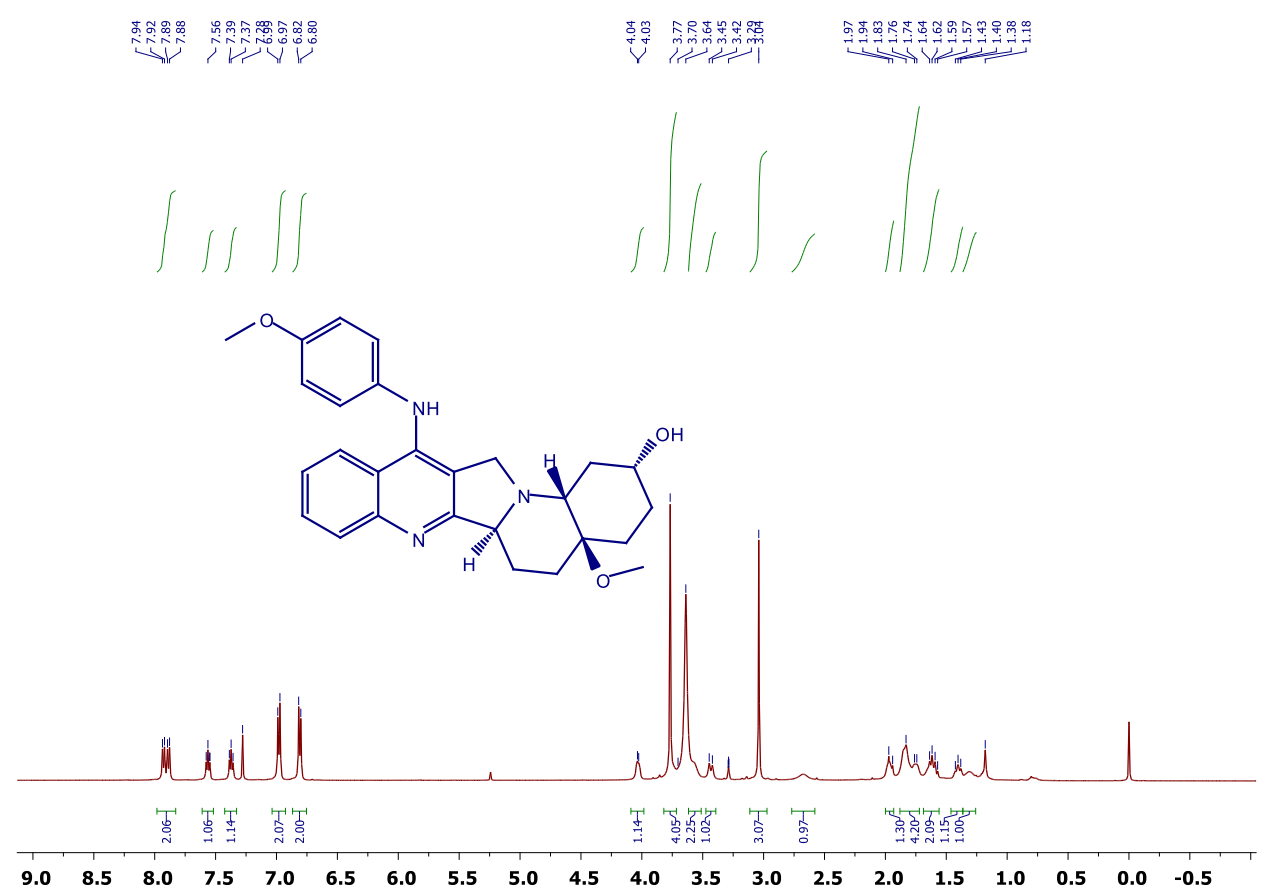

Figure S70 ${ }^{1} \mathrm{H}$ NMR $\left(500 \mathrm{MHz}, \mathrm{CDCl}_{3}\right)$ spectrum of (2R,4aR,6aS,14aR)-4a-methoxy-12-((4methoxyphenyl)amino)-1,2,3,4,4a,5,6,6a,13,14a-decahydropyrrolo[1,2-a:3,4-b']diquinolin-2-ol (19b)
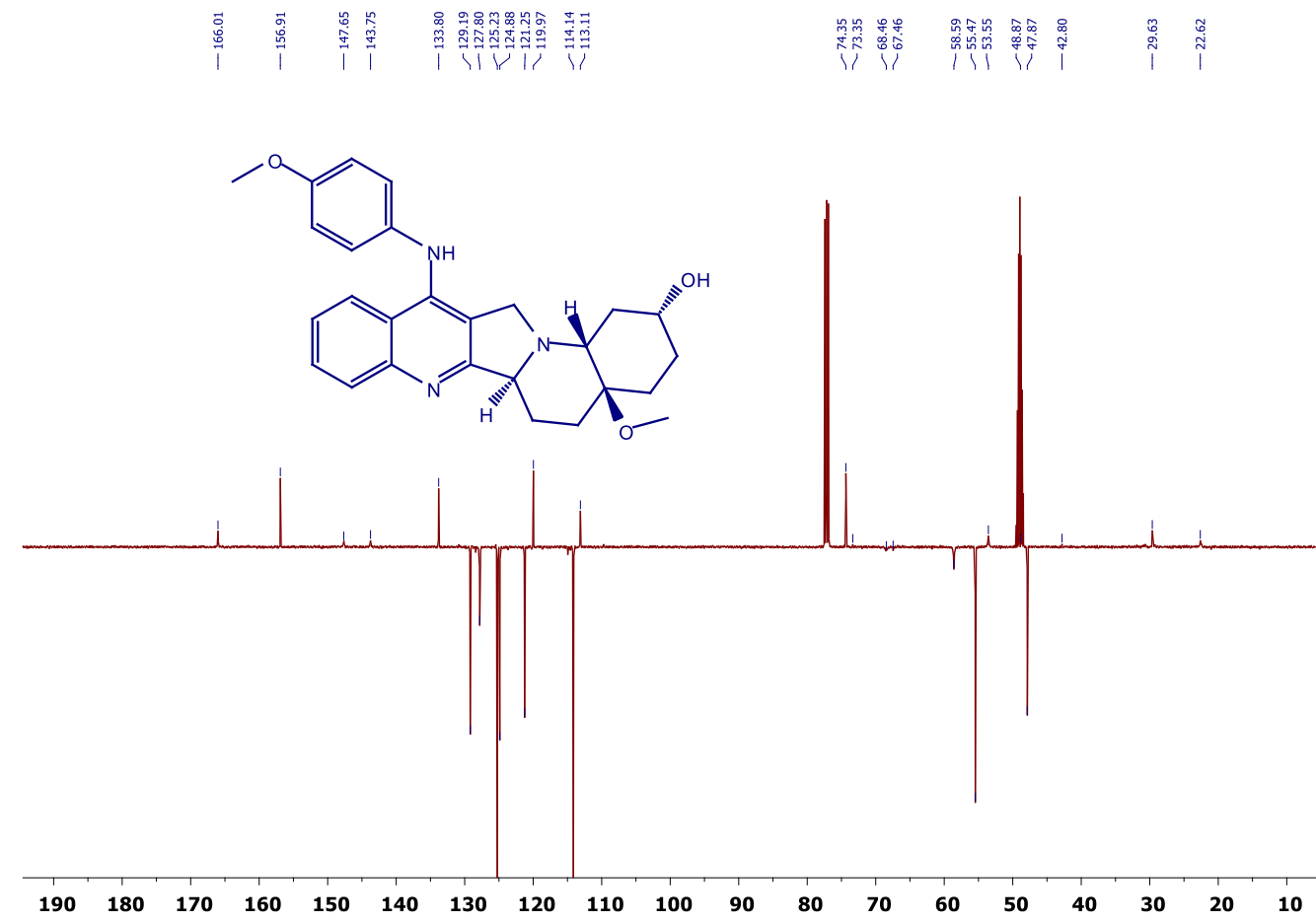

Figure $\mathbf{S 7 1}{ }^{13} \mathrm{C}\left\{{ }^{1} \mathrm{H}\right\}$ APT NMR $\left(125 \mathrm{MHz}, \mathrm{CDCl}_{3}+\mathrm{DMSO}_{-} \mathrm{d}_{6}\right)$ spectrum of (2R,4aR,6aS,14aR)-4a-methoxy-12-((4-methoxyphenyl)amino)-1,2,3,4,4a,5,6,6a, 13,14adecahydropyrrolo[1,2-a:3,4-b']diquinolin-2-ol (19b) 


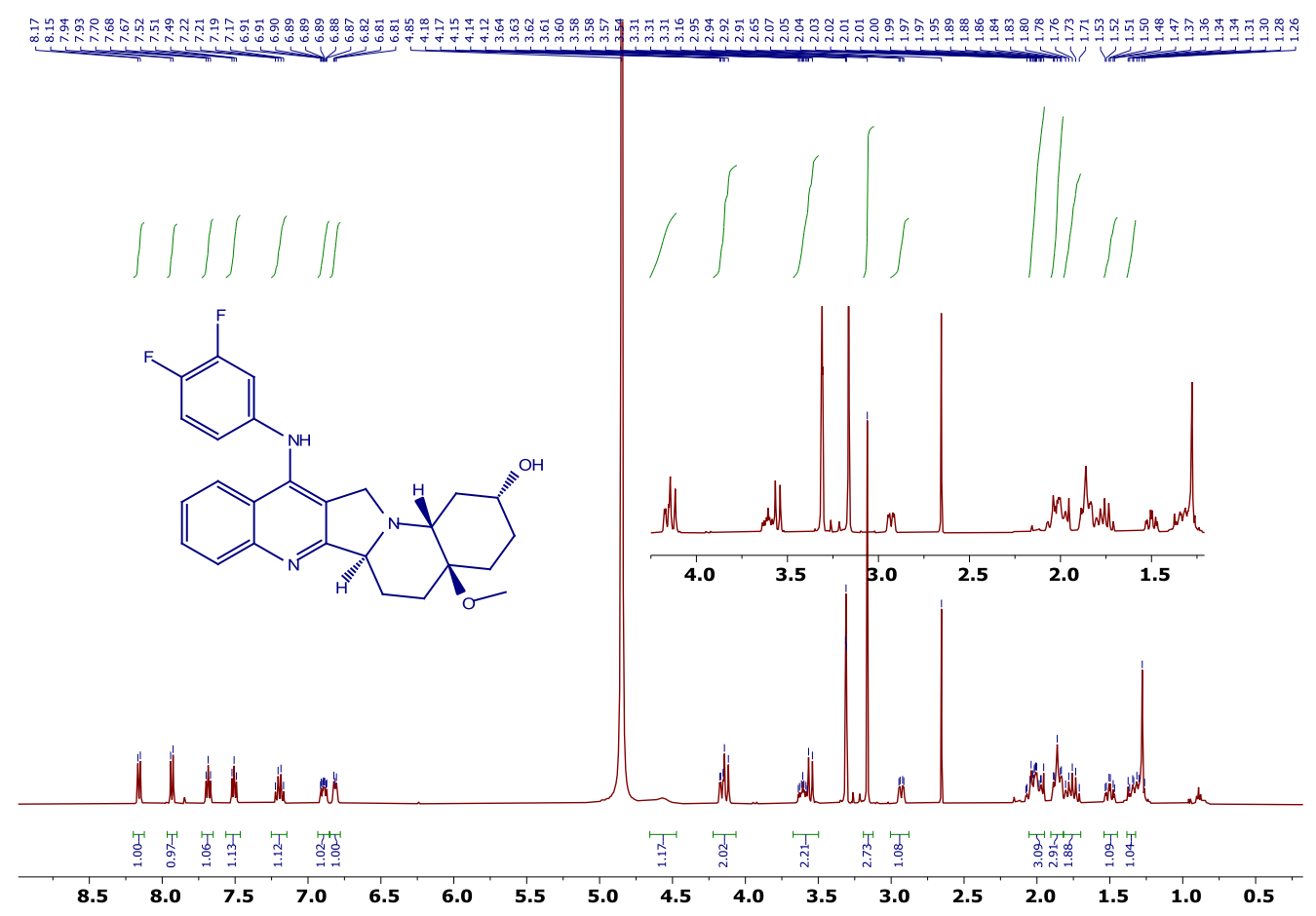

Figure S74 ${ }^{1} \mathrm{H}$ NMR $\left(500 \mathrm{MHz}, \mathrm{CD}_{3} \mathrm{OD}\right)$ spectrum of $(2 \mathrm{R}, 4 \mathrm{aR}, 6 \mathrm{aS}, 14 \mathrm{aR})-12-((3,4-$ difluorophenyl)amino)-4a-methoxy-1,2,3,4,4a,5,6,6a,13,14a-decahydropyrrolo[1,2-a:3,4b']diquinolin-2-ol (19c)

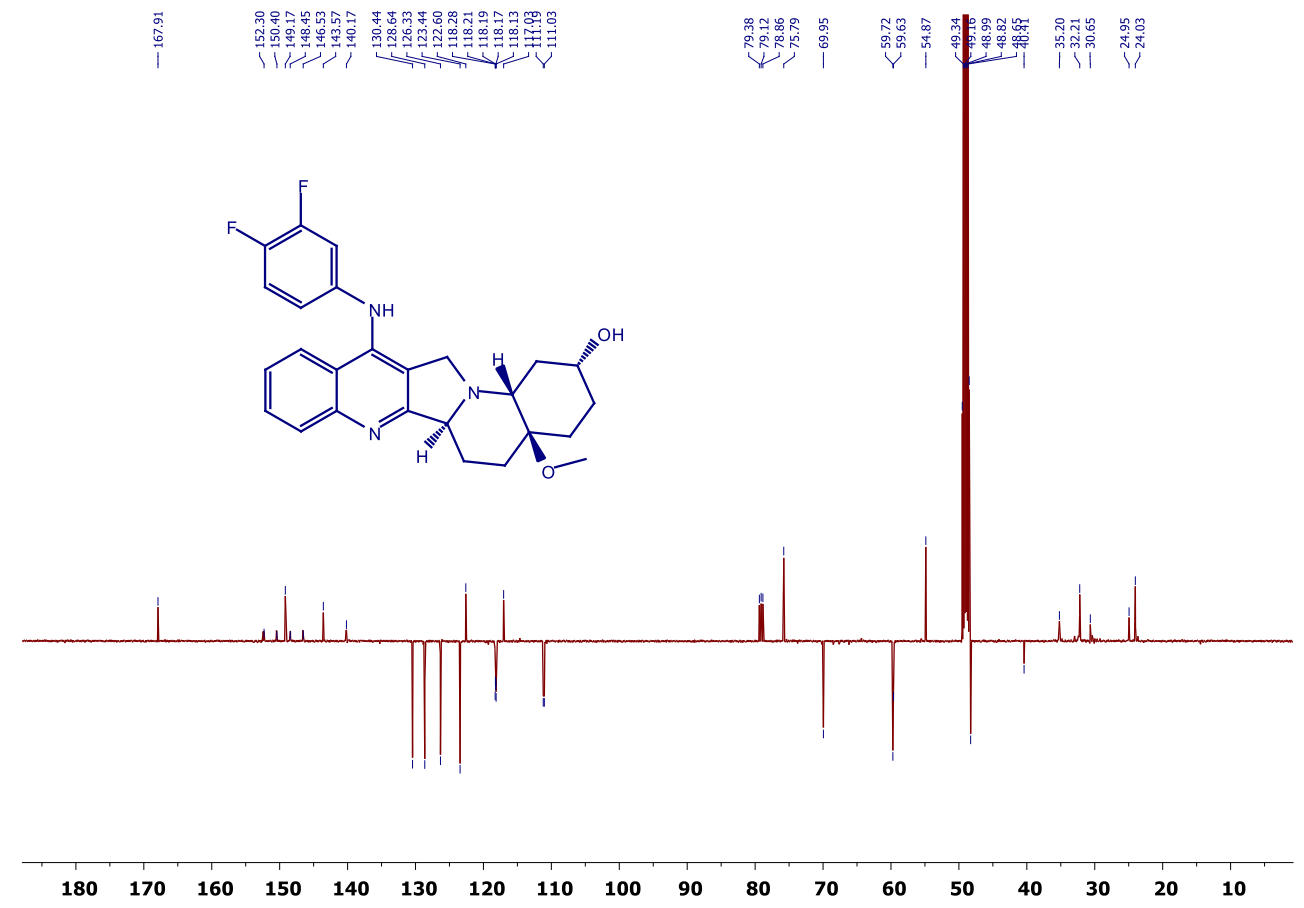

Figure S75 ${ }^{13} \mathrm{C}\left\{{ }^{1} \mathrm{H}\right\}$ APT NMR (125 MHz, CD $\left.{ }_{3} \mathrm{OD}\right)$ spectrum of $(2 \mathrm{R}, 4 \mathrm{aR}, 6 \mathrm{aS}, 14 \mathrm{aR})-12-((3,4-$ difluorophenyl)amino)-4a-methoxy-1,2,3,4,4a,5,6,6a,13,14a-decahydropyrrolo[1,2-a:3,4-

b']diquinolin-2-ol (19c) 


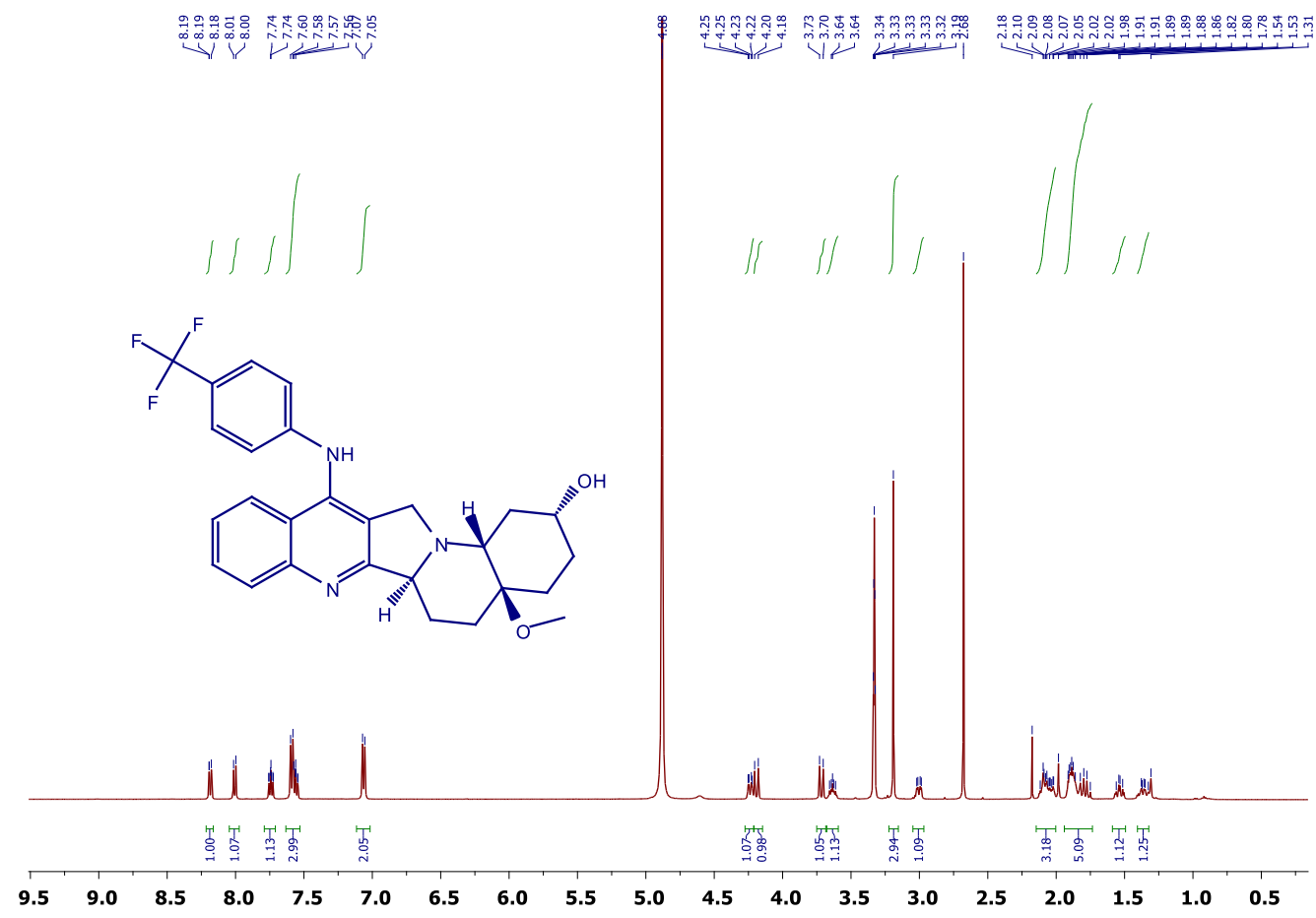

Figure S72 ${ }^{1} \mathrm{H}$ NMR (500 MHz, CD $\left.3 \mathrm{OD}\right)$ spectrum of (2R,4aR,6aS, 14aR)-4a-methoxy-12-((4methoxyphenyl)amino)-1,2,3,4,4a,5,6,6a,13,14a-decahydropyrrolo[1,2-a:3,4-b']diquinolin-2-ol (19d)
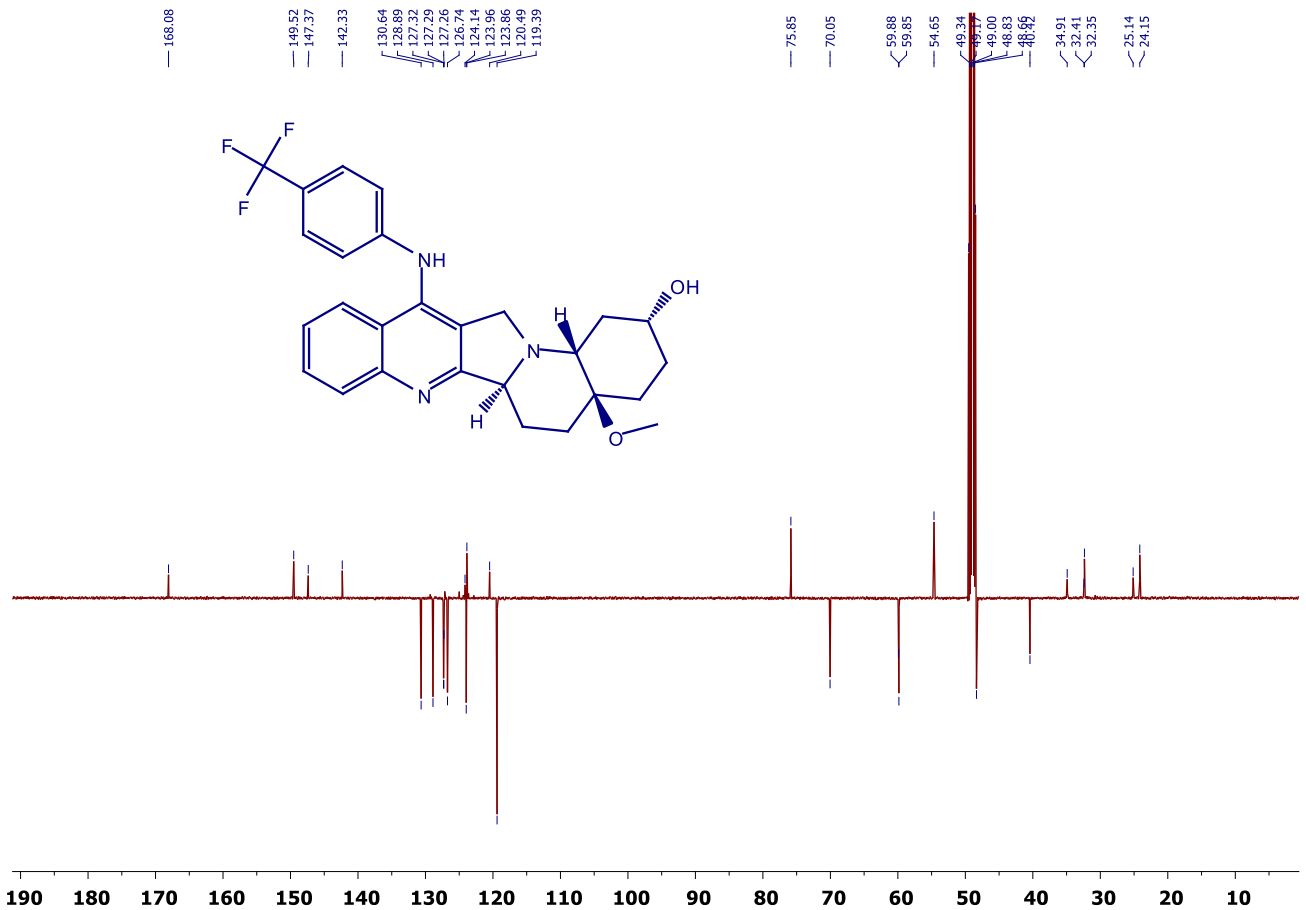

Figure S73 ${ }^{13} \mathrm{C}\left\{{ }^{1} \mathrm{H}\right\}$ APT NMR (125 MHz, CD $\left.3 \mathrm{OD}\right)$ spectrum of $(2 \mathrm{R}, 4 \mathrm{aR}, 6 \mathrm{aS}, 14 \mathrm{aR})-4 \mathrm{a}-$ methoxy-12-((4-methoxyphenyl)amino)-1,2,3,4,4a,5,6,6a,13,14a-decahydropyrrolo[1,2-a:3,4b']diquinolin-2-ol (19d) 


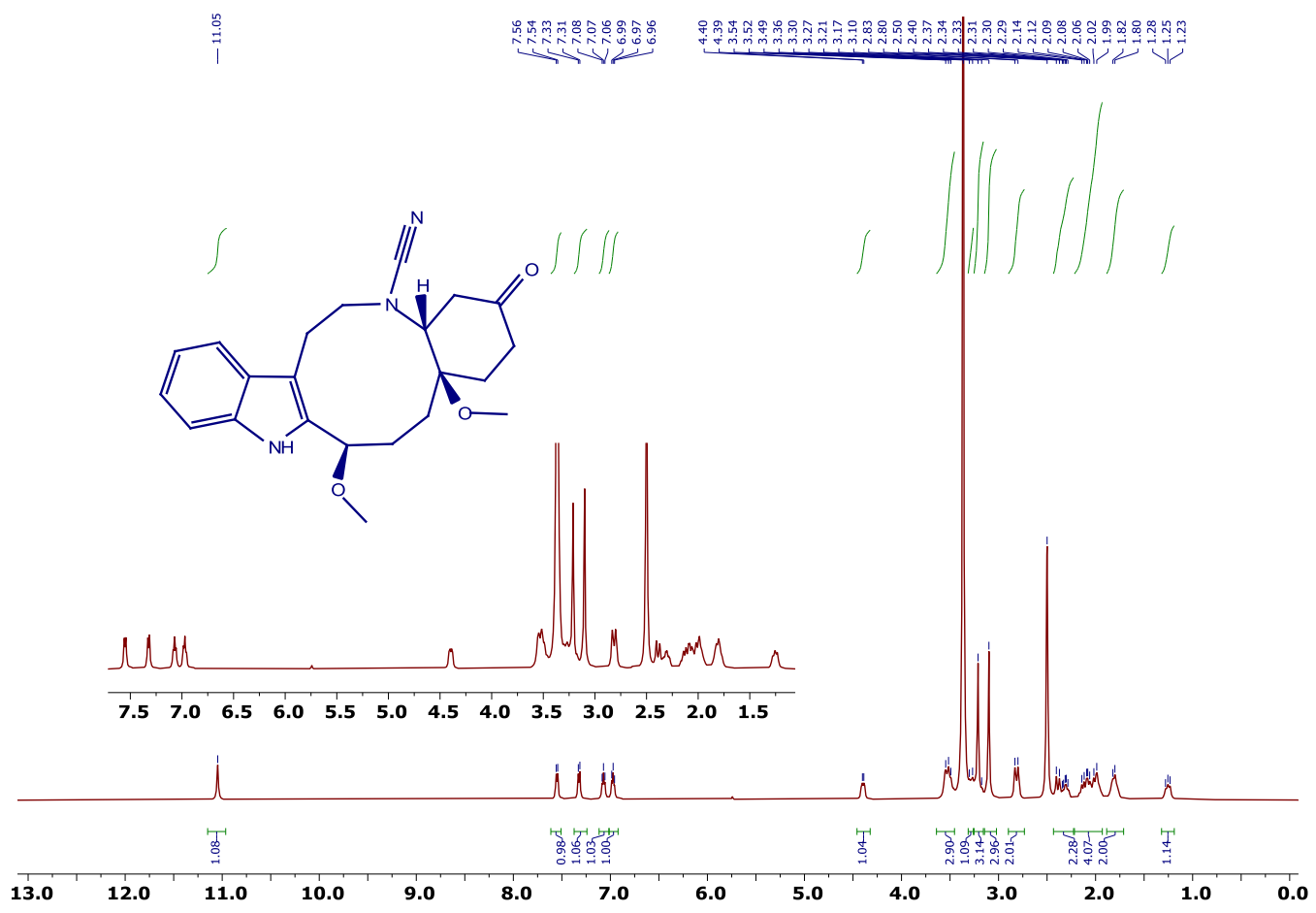

Figure S76 ${ }^{1} \mathrm{H}$ NMR (500 MHz, DMSO-d $)$ spectrum of (4aR,13R,15aR)-13,15a-dimethoxy-3oxo-1,2,3,4,4a,6,7,12,13,14,15,15a-dodecahydro-5H-benzo[9,10]azecino[5,4-b]indole-5carbonitrile (20a)
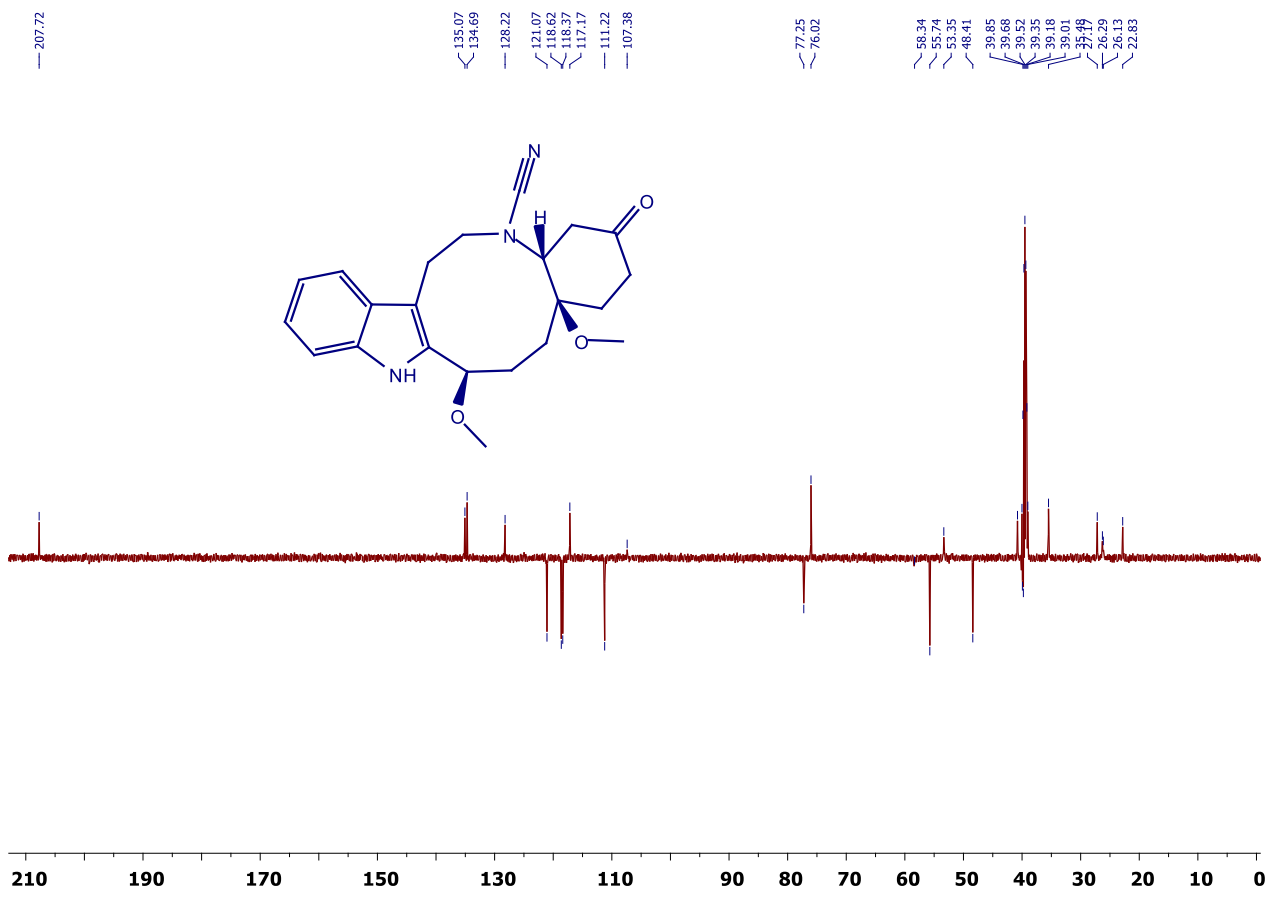

Figure S77 ${ }^{13} \mathrm{C}\left\{{ }^{1} \mathrm{H}\right\}$ APT NMR (125 MHz, DMSO-d $)$ spectrum of (4aR,13R,15aR)-13,15adimethoxy-3-oxo-1,2,3,4,4a,6,7,12,13,14,15,15a-dodecahydro-5H-benzo[9,10]azecino[5,4b]indole-5-carbonitrile (20a) 


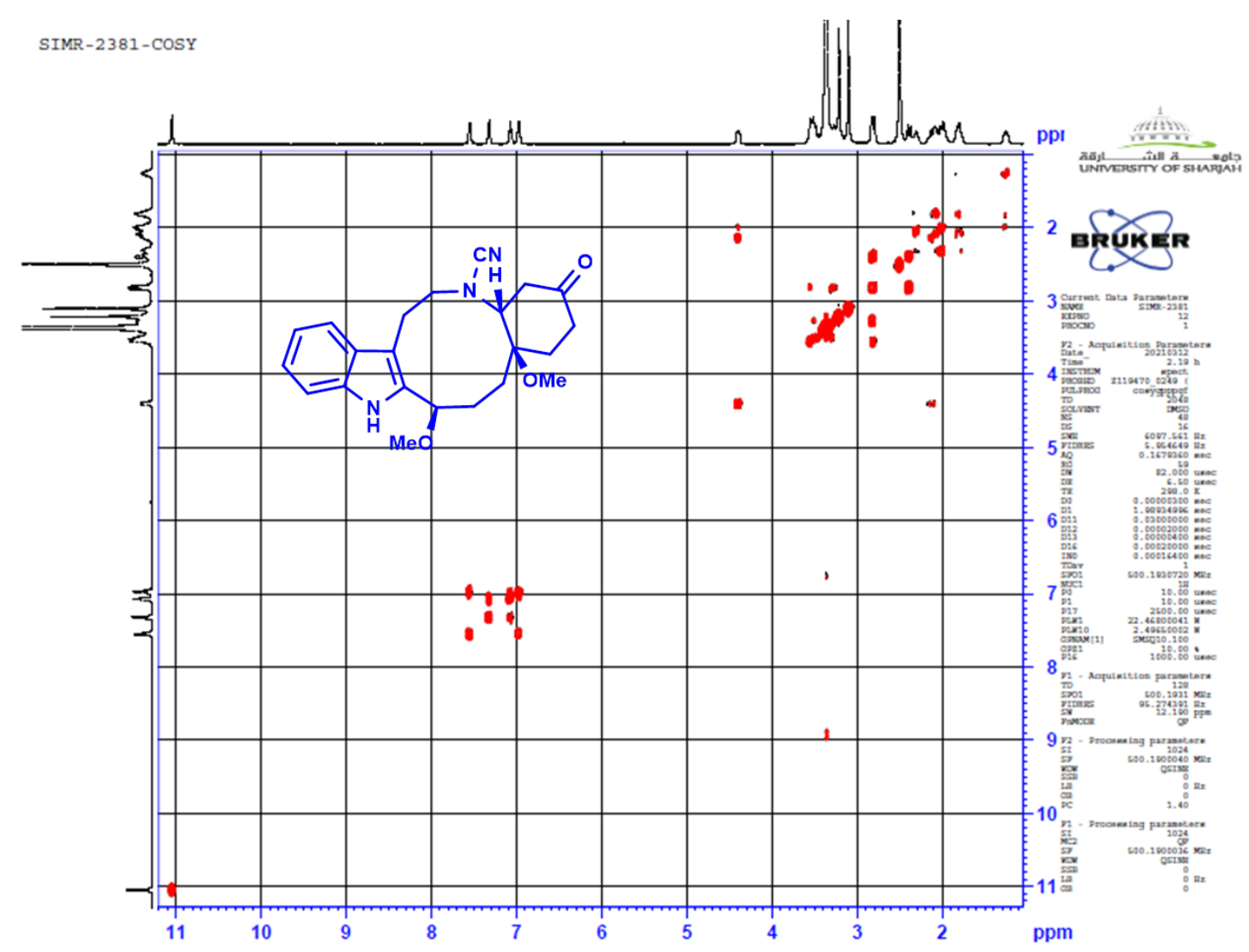

Figure S78 COSY NMR (500 MHz, DMSO-d $)$ spectrum of (4aR,13R,15aR)-13,15adimethoxy-3-oxo-1,2,3,4,4a,6,7,12,13,14,15,15a-dodecahydro-5H-benzo[9,10]azecino[5,4b]indole-5-carbonitrile (20a) 


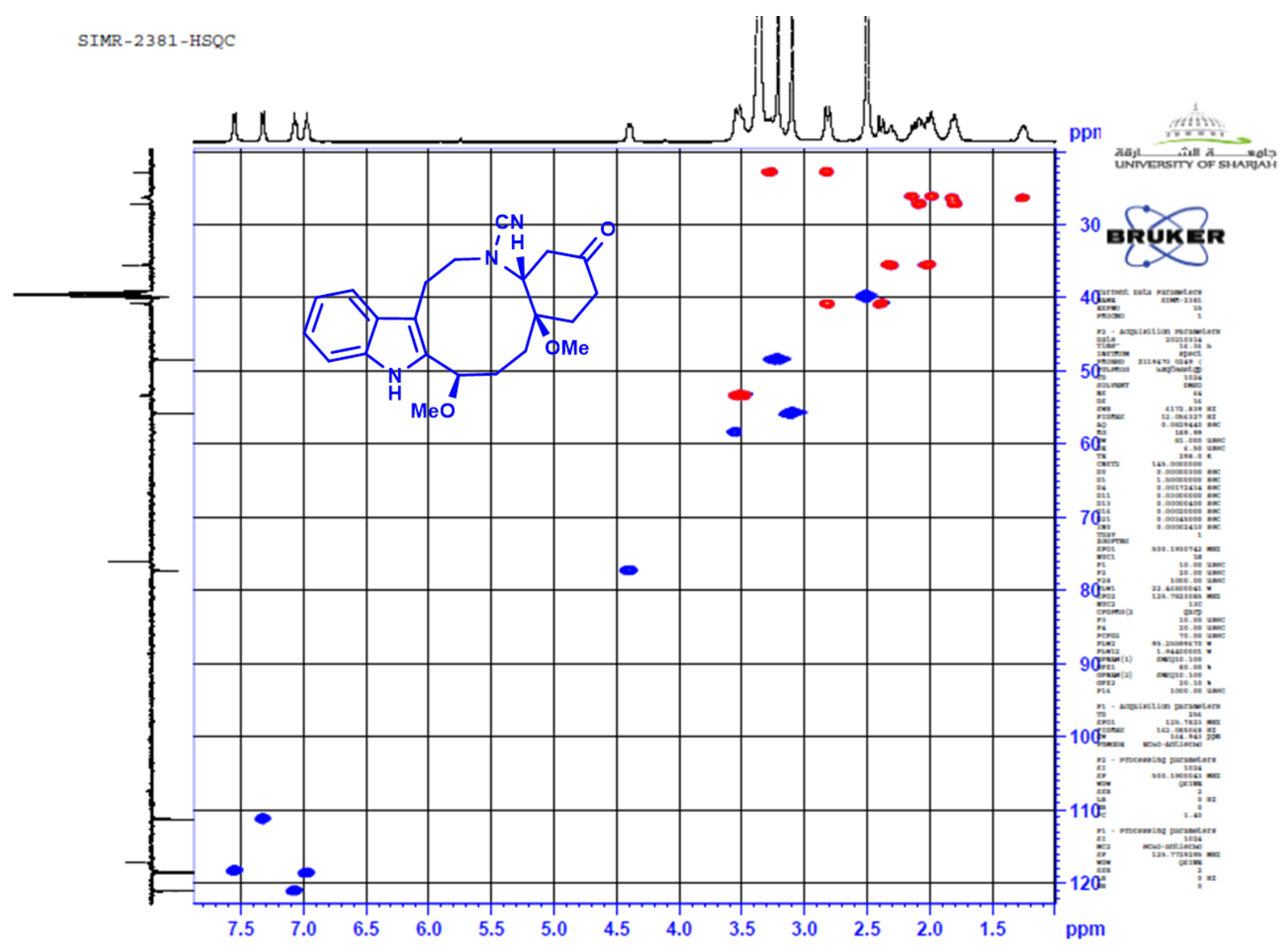

Figure S79 HSQC NMR (500 MHz, DMSO-d ${ }_{6}$ ) spectrum of (4aR,13R,15aR)-13,15adimethoxy-3-oxo-1,2,3,4,4a,6,7,12,13,14,15,15a-dodecahydro-5H-benzo[9,10]azecino[5,4b]indole-5-carbonitrile (20a) 


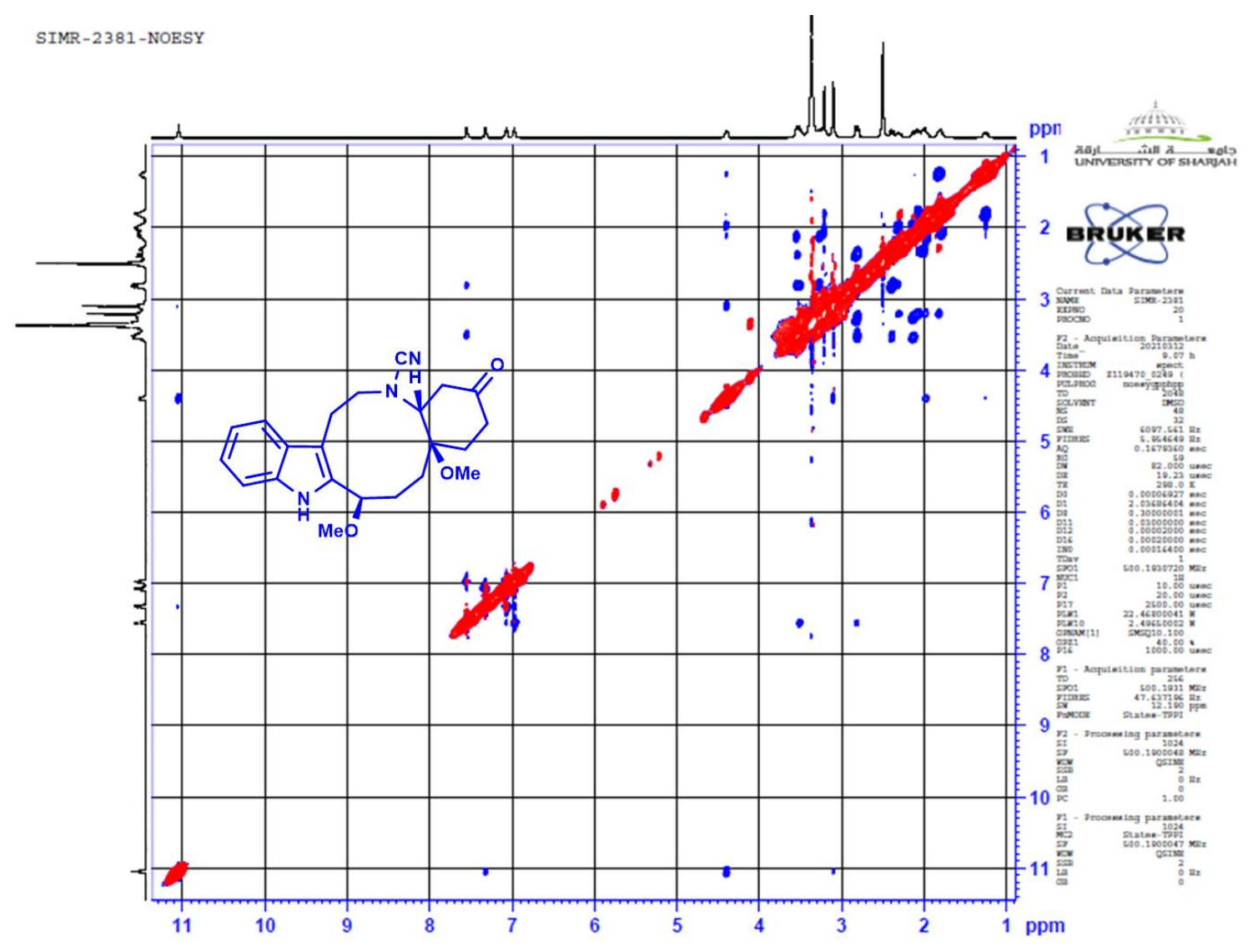

Figure S80 NOESY NMR (500 MHz, DMSO-d $)$ spectrum of (4aR,13R,15aR)-13,15adimethoxy-3-oxo-1,2,3,4,4a,6,7,12,13,14,15,15a-dodecahydro-5H-benzo[9,10]azecino[5,4b]indole-5-carbonitrile (20a) 


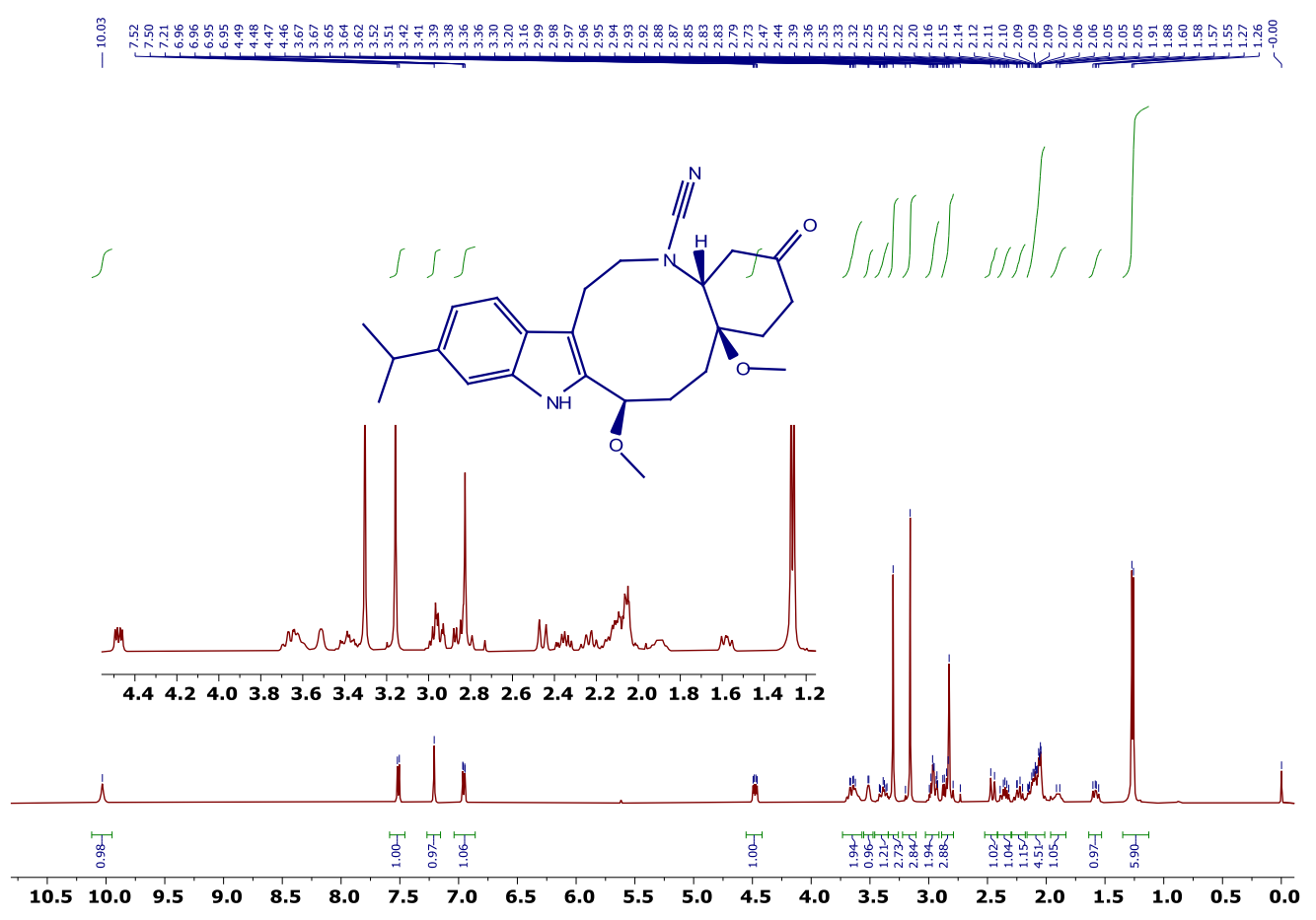

Figure S81 ${ }^{1} \mathrm{H}$ NMR (500 MHz, Acetone-d 6 ) spectrum of (4aR,13R,15aR)-10-isopropyl-13,15adimethoxy-3-oxo-1,2,3,4,4a,6,7,12,13,14,15,15a-dodecahydro-5H-benzo[9,10]azecino[5,4b]indole-5-carbonitrile (20b)
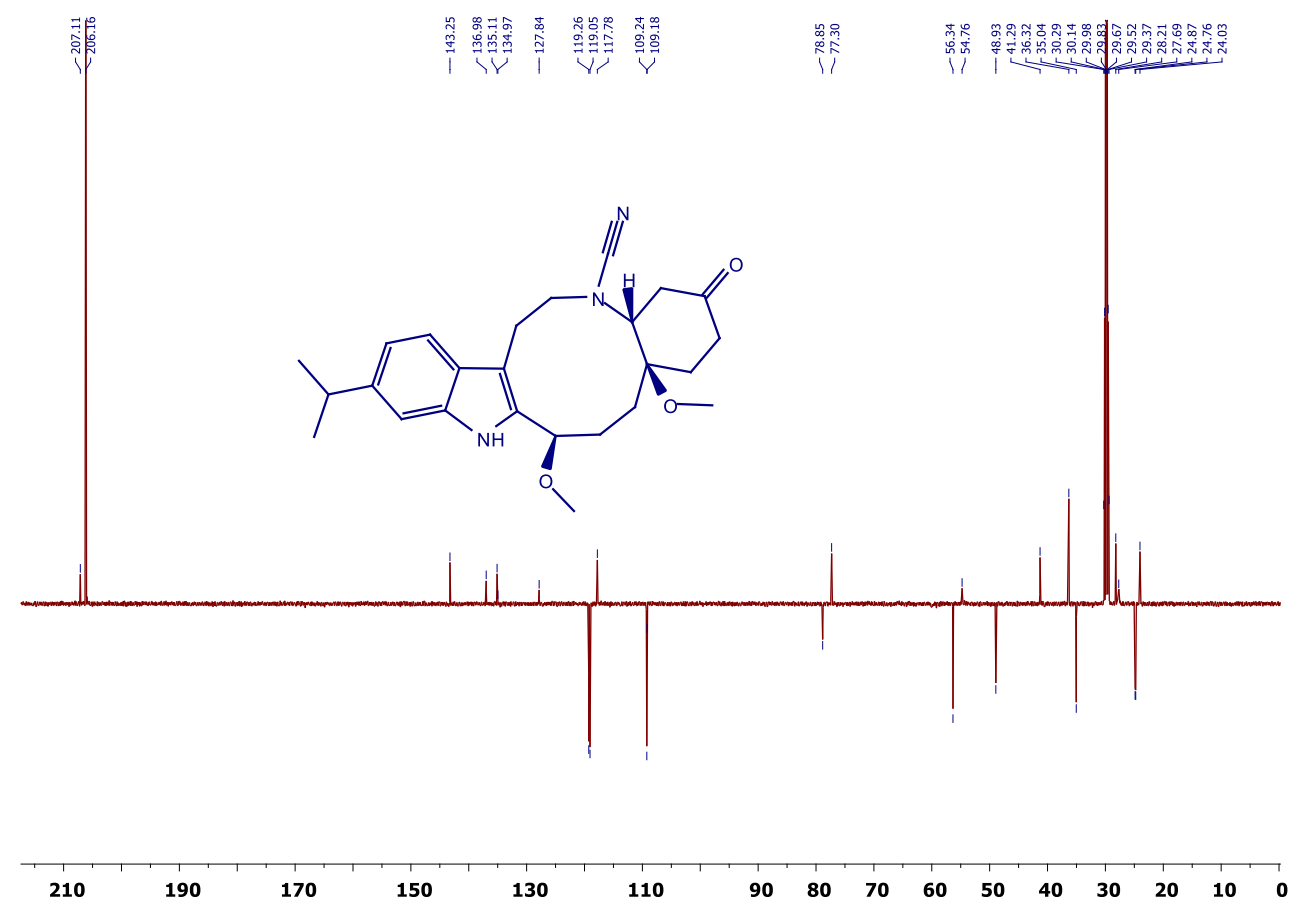

Figure S82 ${ }^{13} \mathrm{C}\left\{{ }^{1} \mathrm{H}\right\}$ APT NMR (125 MHz, Acetone-d 6 ) spectrum of (4aR,13R,15aR)-10isopropyl-13,15a-dimethoxy-3-oxo-1,2,3,4,4a,6,7,12,13,14,15,15a-dodecahydro-5Hbenzo[9,10]azecino[5,4-b]indole-5-carbonitrile (20b) 


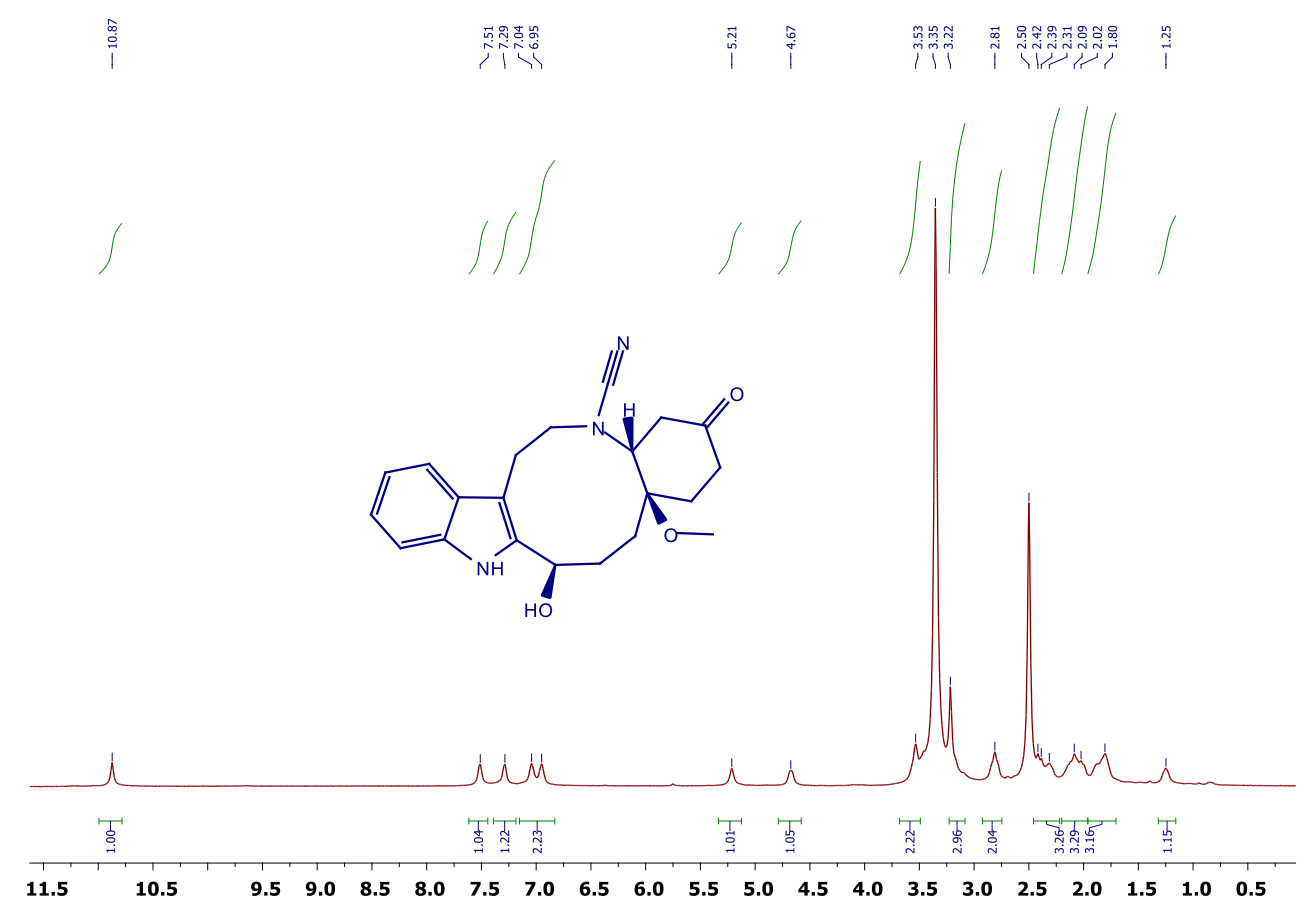

Figure S83 ${ }^{1} \mathrm{H}$ NMR (500 MHz, DMSO-d $)$ spectrum of (4aR,13R,15aR)-13-hydroxy-15amethoxy-3-oxo-1,2,3,4,4a,6,7,12,13,14,15,15a-dodecahydro-5H-benzo[9,10]azecino[5,4b]indole-5-carbonitrile (21)

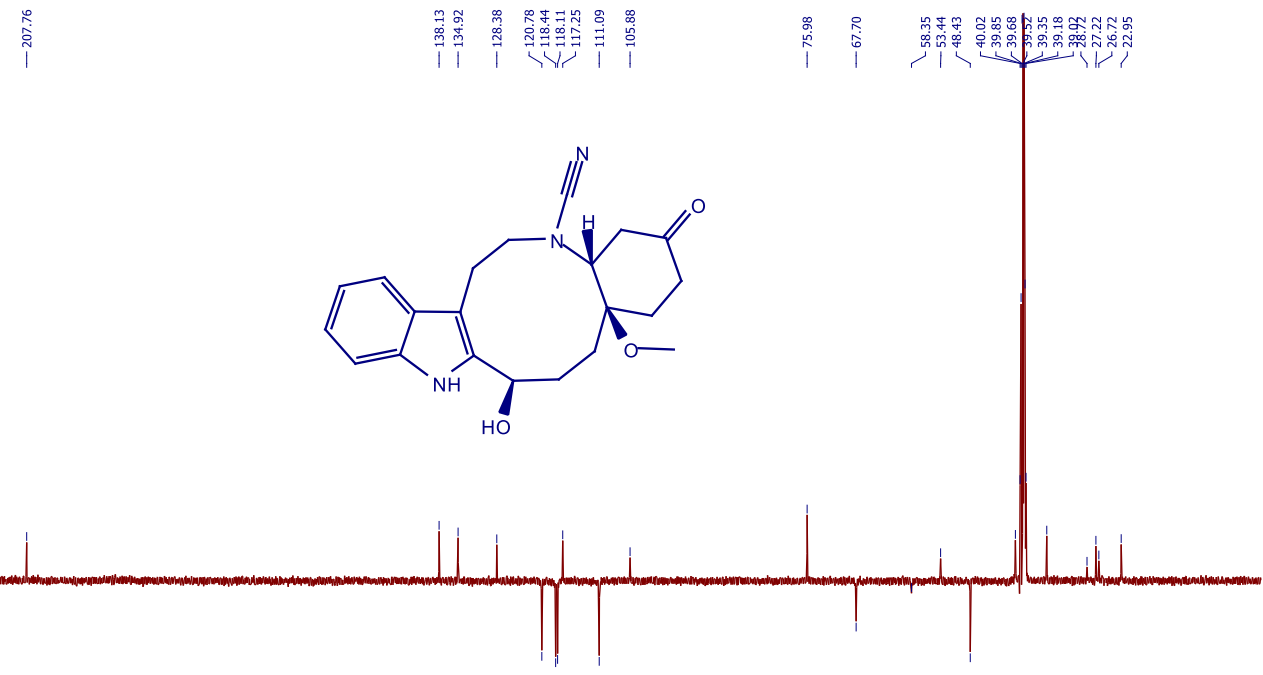

\begin{tabular}{lllllllllllllllll}
\hline 210 & 190 & 170 & 150 & 130 & 110 & 90 & 80 & 70 & 60 & 50 & 40 & 30 & 20 & 10 & 0
\end{tabular}

Figure S84 ${ }^{13} \mathrm{C}\left\{{ }^{1} \mathrm{H}\right\}$ APT NMR (125 MHz, DMSO-d 6 ) spectrum of (4aR,13R,15aR)-13hydroxy-15a-methoxy-3-oxo-1,2,3,4,4a,6,7,12,13,14,15,15a-dodecahydro-5Hbenzo[9,10]azecino[5,4-b]indole-5-carbonitrile (21) 


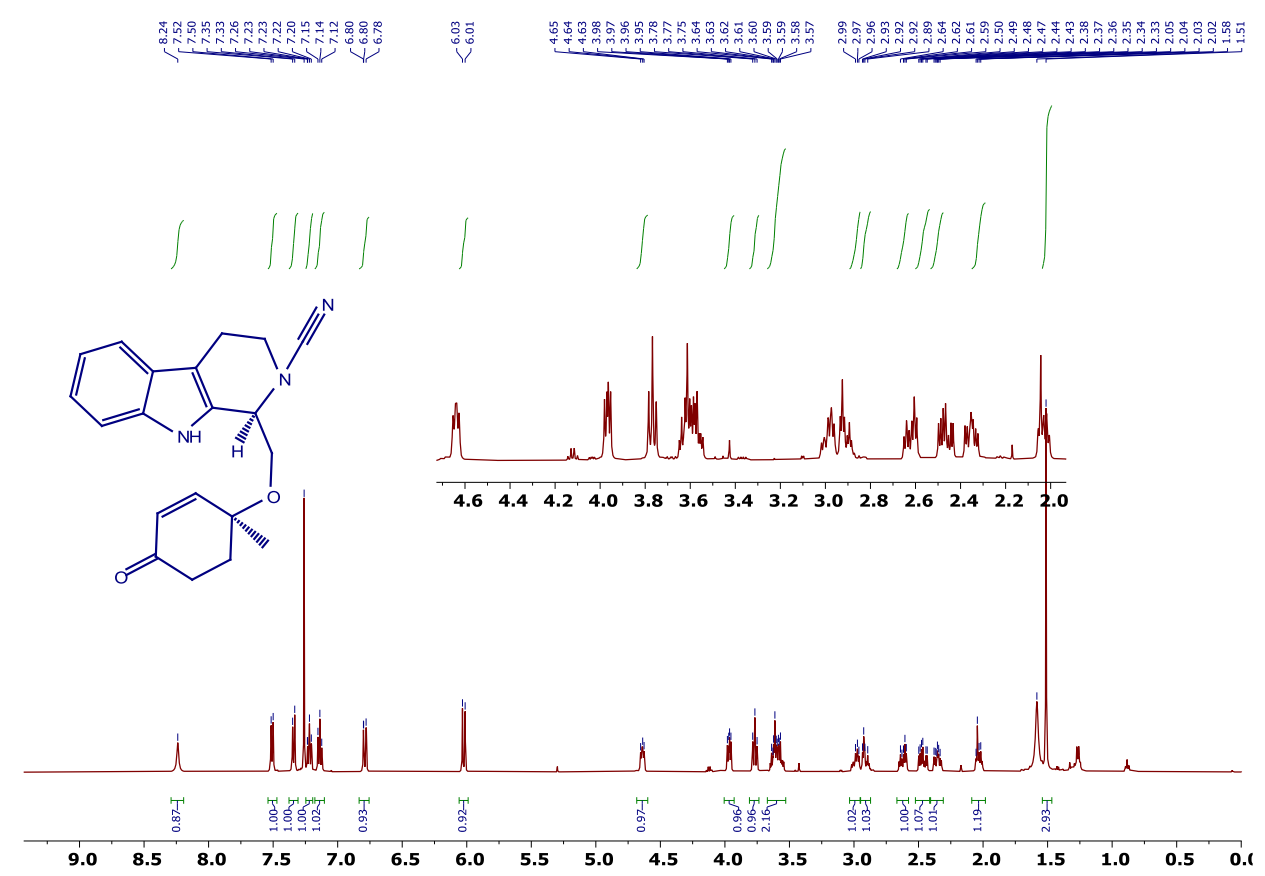

Figure S85 ${ }^{1} \mathrm{H}$ NMR $\left(500 \mathrm{MHz}, \mathrm{CDCl}_{3}\right)$ spectrum of methyl (E)-4-((R)-1-((((S)-1-methyl-4oxocyclohex-2-en-1-yl)oxy)methyl)-1,3,4,9-tetrahydro-2H-pyrido[3,4-b]indol-2-yl)but-2-enoate (22)

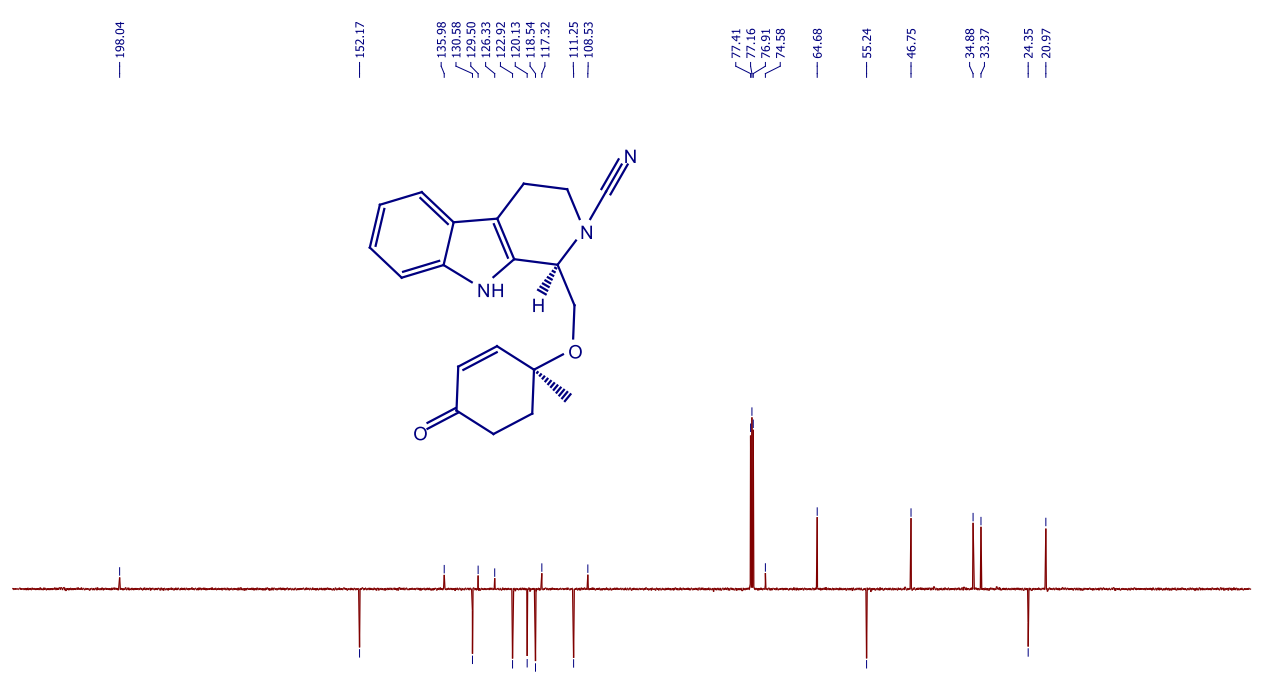

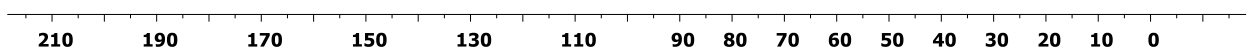

Figure S86 ${ }^{13} \mathrm{C}\left\{{ }^{1} \mathrm{H}\right\}$ APT NMR $\left(125 \mathrm{MHz}, \mathrm{CDCl}_{3}\right)$ spectrum of methyl (E)-4-((R)-1-((((S)-1methyl-4-oxocyclohex-2-en-1-yl)oxy)methyl)-1,3,4,9-tetrahydro-2H-pyrido[3,4-b]indol-2yl)but-2-enoate (22) 


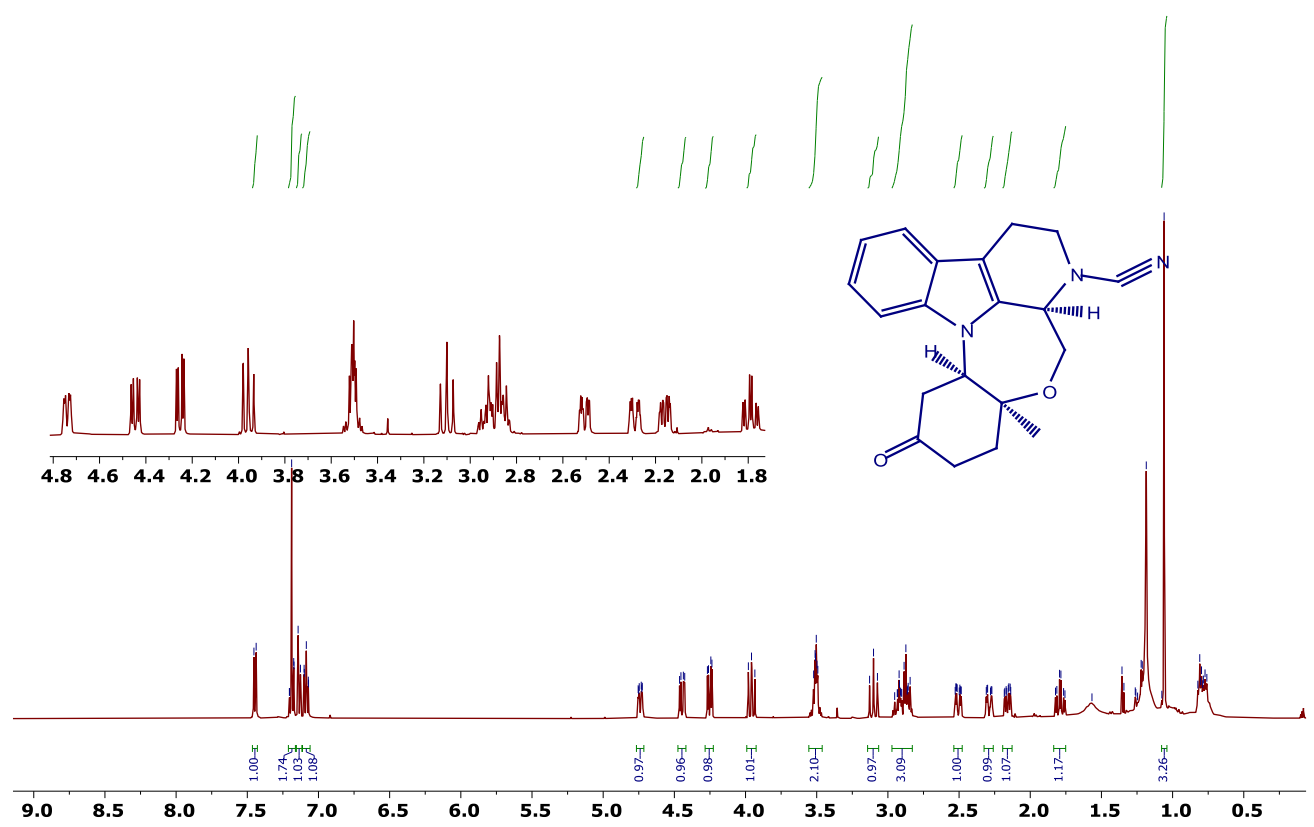

Figure S87 ${ }^{1} \mathrm{H}$ NMR $\left(500 \mathrm{MHz}, \mathrm{CDCl}_{3}\right)$ spectrum of (3aR,5aS,9aR)-5a-methyl-8-oxo1,2,3a,4,5a,6,7,8,9,9a-decahydro-3H-5-oxa-3,9b-diazabenzo[4,5]cyclohepta[1,2,3-jk]fluorene-3carbonitrile (23)
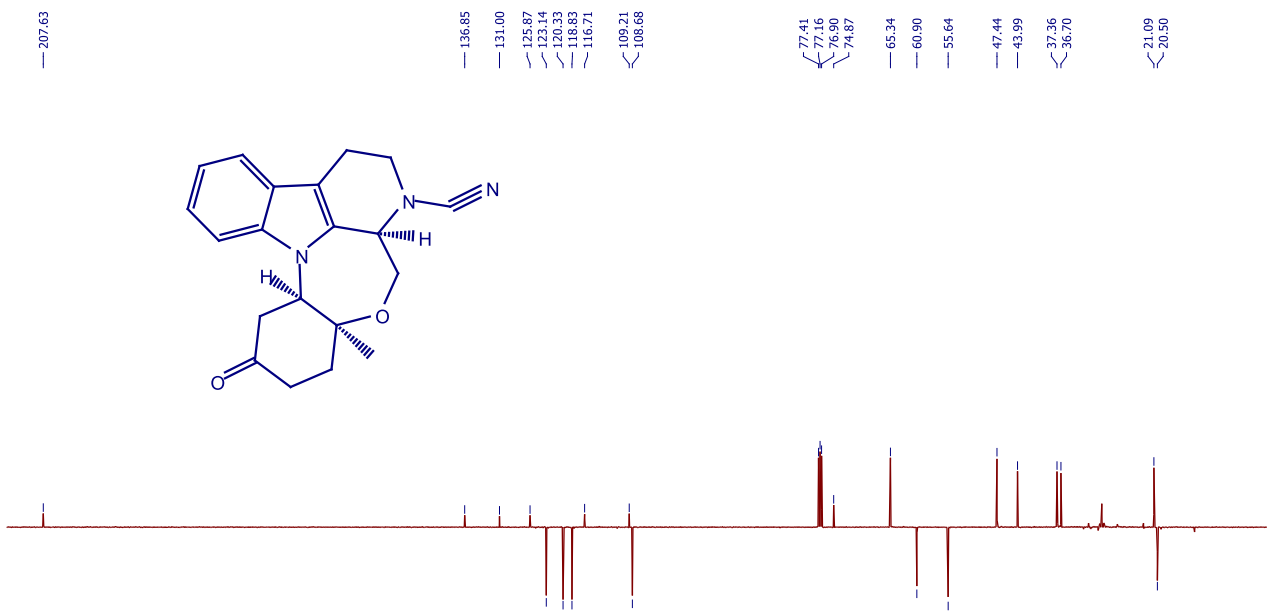

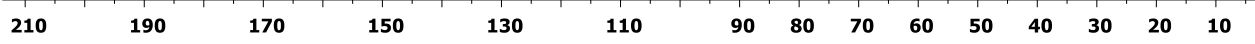

Figure S88 ${ }^{13} \mathrm{C}\left\{{ }^{1} \mathrm{H}\right\}$ APT NMR (125 MHz, CDCl 3 ) spectrum of (3aR,5aS,9aR)-5a-methyl-8oxo-1,2,3a,4,5a,6,7,8,9,9a-decahydro-3H-5-oxa-3,9b-diazabenzo[4,5]cyclohepta[1,2,3jk]fluorene-3-carbonitrile (23) 


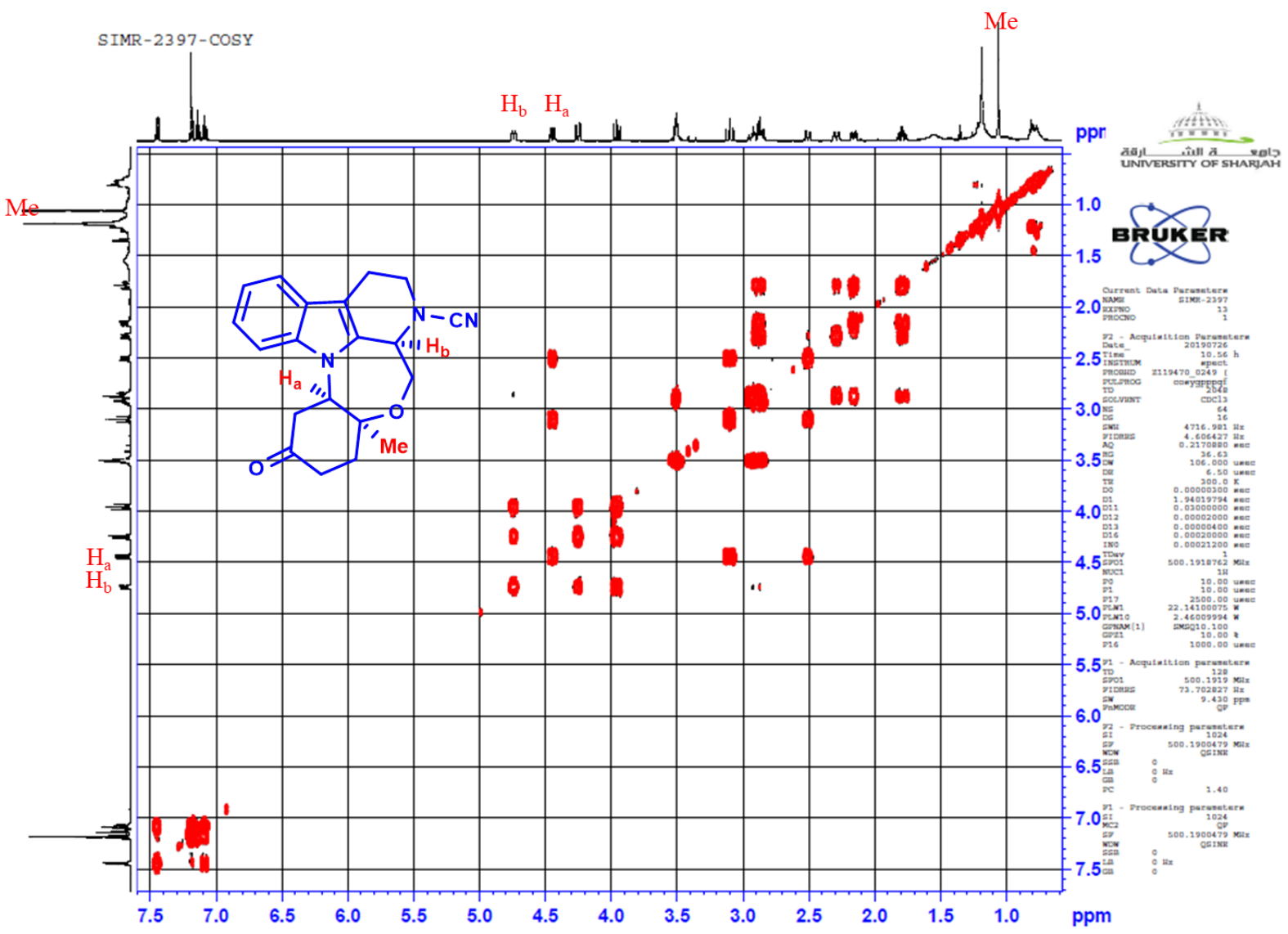

Figure S89 COSY NMR (500 MHz, $\left.\mathrm{CDCl}_{3}\right)$ spectrum of (3aR,5aS,9aR)-5a-methyl-8-oxo1,2,3a,4,5a,6,7,8,9,9a-decahydro-3H-5-oxa-3,9b-diazabenzo[4,5]cyclohepta[1,2,3-jk]fluorene-3carbonitrile (23) 


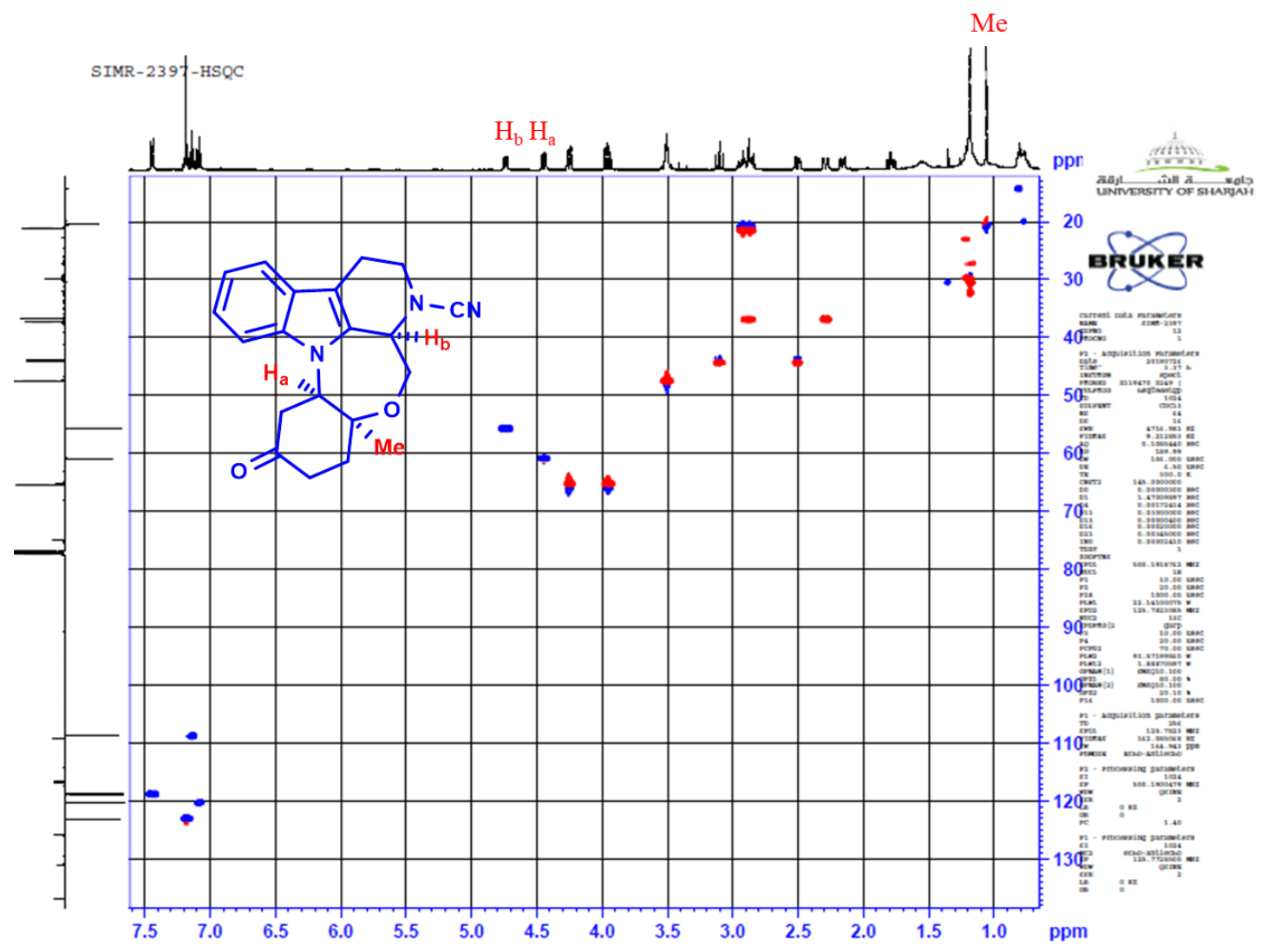

Figure S90 HSQC NMR ( $500 \mathrm{MHz}, \mathrm{CDCl}_{3}$ ) spectrum of (3aR,5aS,9aR)-5a-methyl-8-oxo1,2,3a,4,5a,6,7,8,9,9a-decahydro-3H-5-oxa-3,9b-diazabenzo[4,5]cyclohepta[1,2,3-jk]fluorene-3carbonitrile (23) 


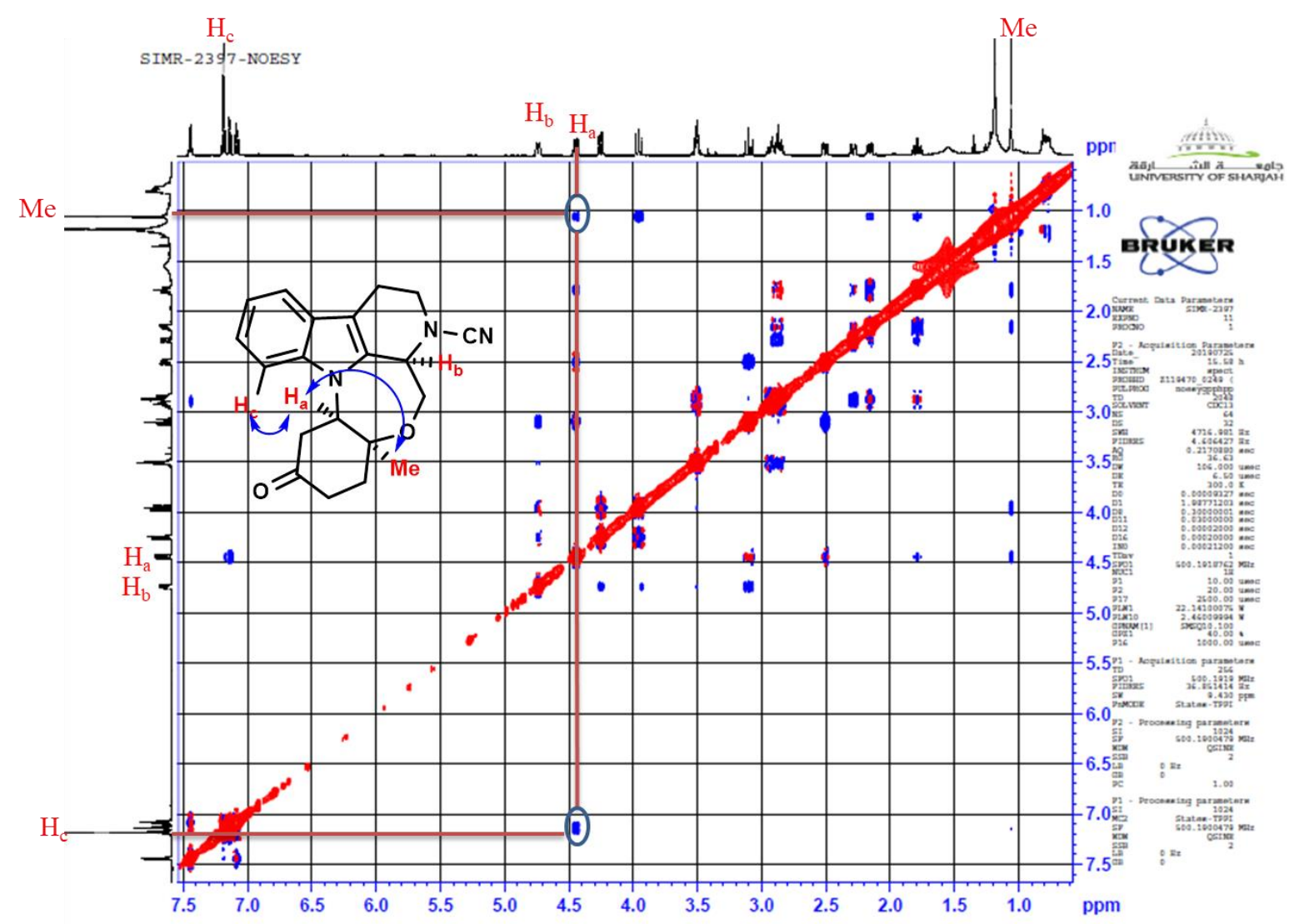

Figure S91 NOESY NMR (500 MHz, $\left.\mathrm{CDCl}_{3}\right)$ spectrum of (3aR,5aS,9aR)-5a-methyl-8-oxo1,2,3a,4,5a,6,7,8,9,9a-decahydro-3H-5-oxa-3,9b-diazabenzo[4,5]cyclohepta[1,2,3-jk]fluorene-3carbonitrile $(\mathbf{2 3})$ 


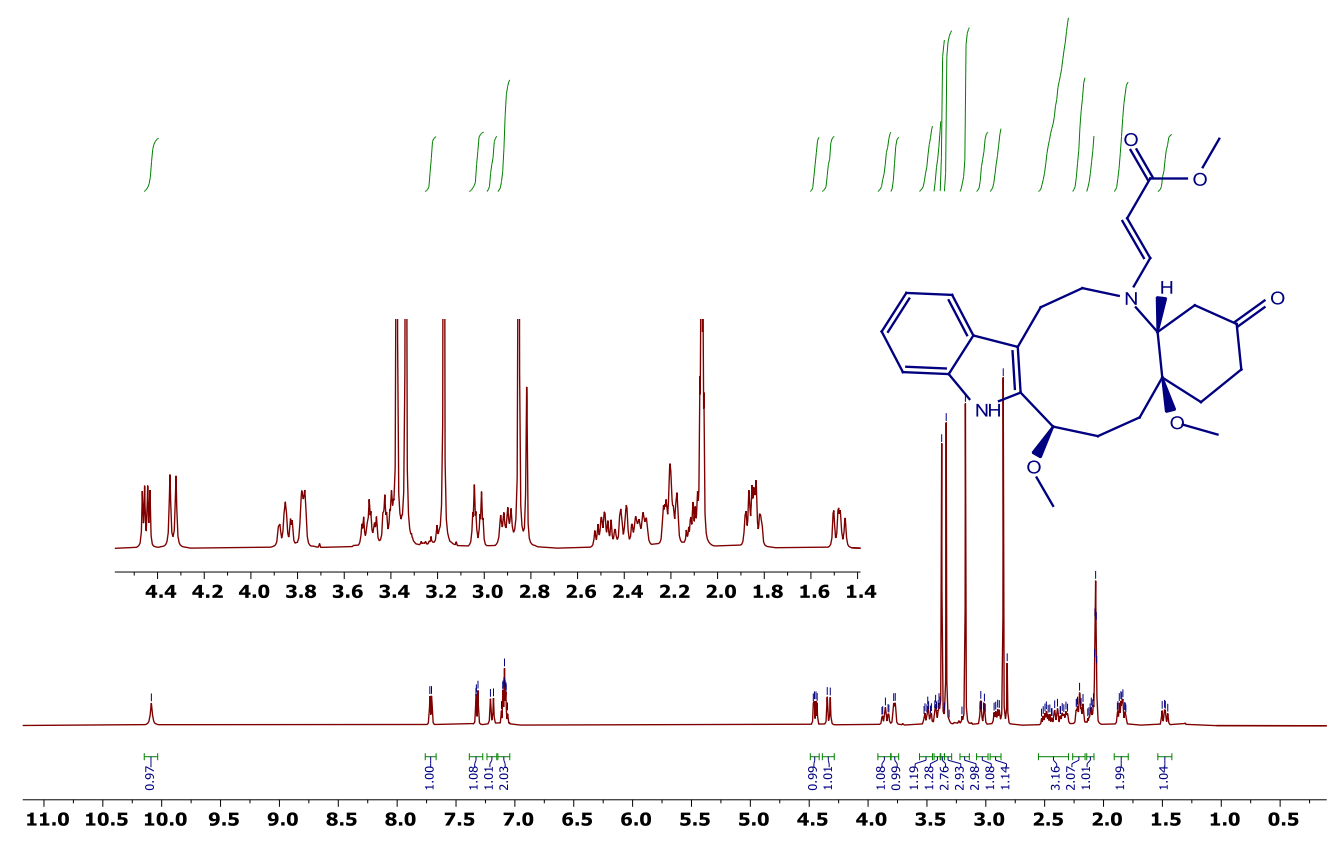

Figure S92 ${ }^{1} \mathrm{H}$ NMR (500 MHz, Acetone-d 6 ) spectrum of methyl (E)-3-((4aR,13R,15aR)13,15a-dimethoxy-3-oxo-1,2,3,4,4a,6,7,12,13,14,15,15a-dodecahydro-5Hbenzo[9,10]azecino[5,4-b]indol-5-yl)acrylate (25a)

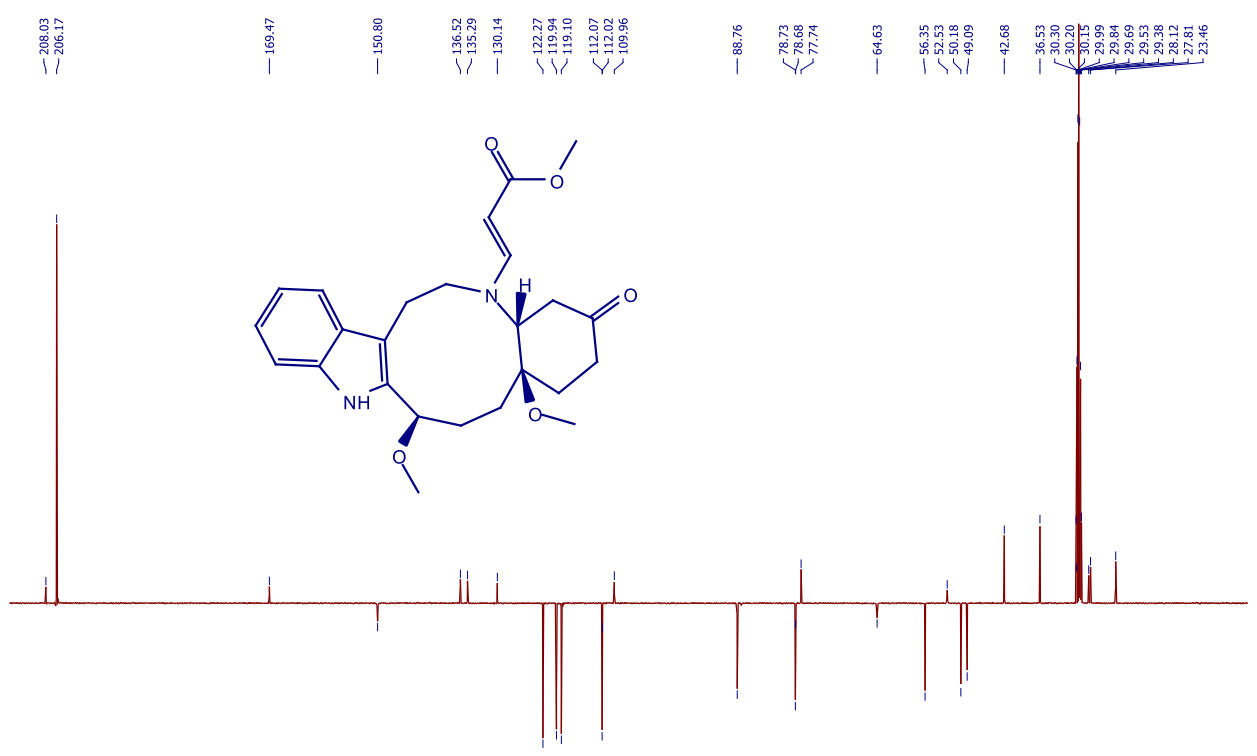

$\begin{array}{lllllllllllllllllllllll}210 & 190 & 170 & 150 & 130 & 110 & 90 & 80 & 70 & 60 & 50 & 40 & 30 & 20 & 10\end{array}$

Figure S93 ${ }^{13} \mathrm{C}\left\{{ }^{1} \mathrm{H}\right\}$ APT NMR (125 MHz, Acetone-d 6 ) spectrum of methyl (E)-3((4aR,13R,15aR)-13,15a-dimethoxy-3-oxo-1,2,3,4,4a,6,7,12,13,14,15,15a-dodecahydro-5Hbenzo[9,10]azecino[5,4-b]indol-5-yl)acrylate (25a) 


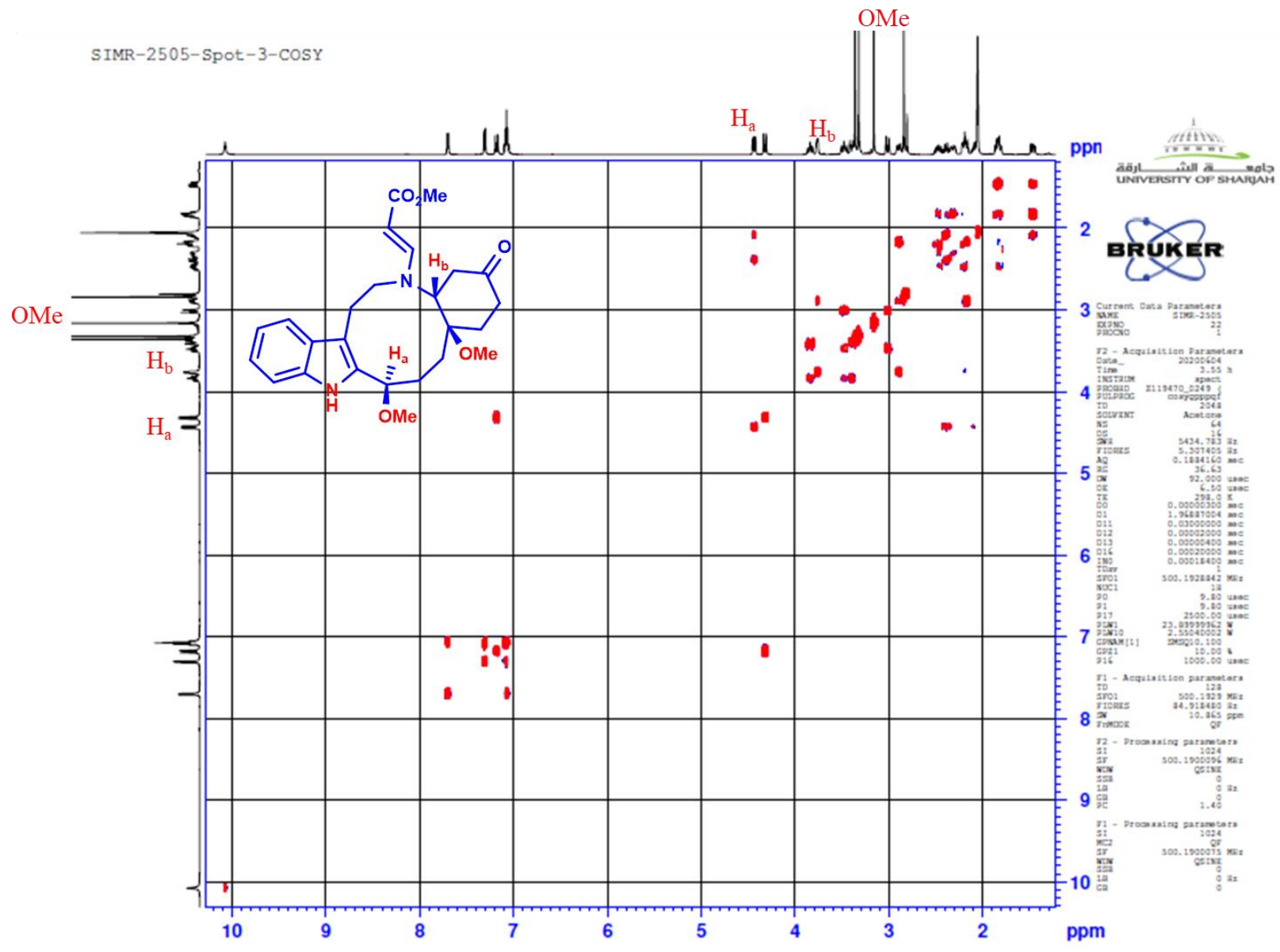

Figure S94 COSY NMR (500 MHz, Acetone-d $\left.)_{6}\right)$ spectrum of methyl (E)-3-((4aR,13R,15aR)13,15a-dimethoxy-3-oxo-1,2,3,4,4a,6,7,12,13,14,15,15a-dodecahydro-5Hbenzo[9,10]azecino[5,4-b]indol-5-yl)acrylate (25a) 


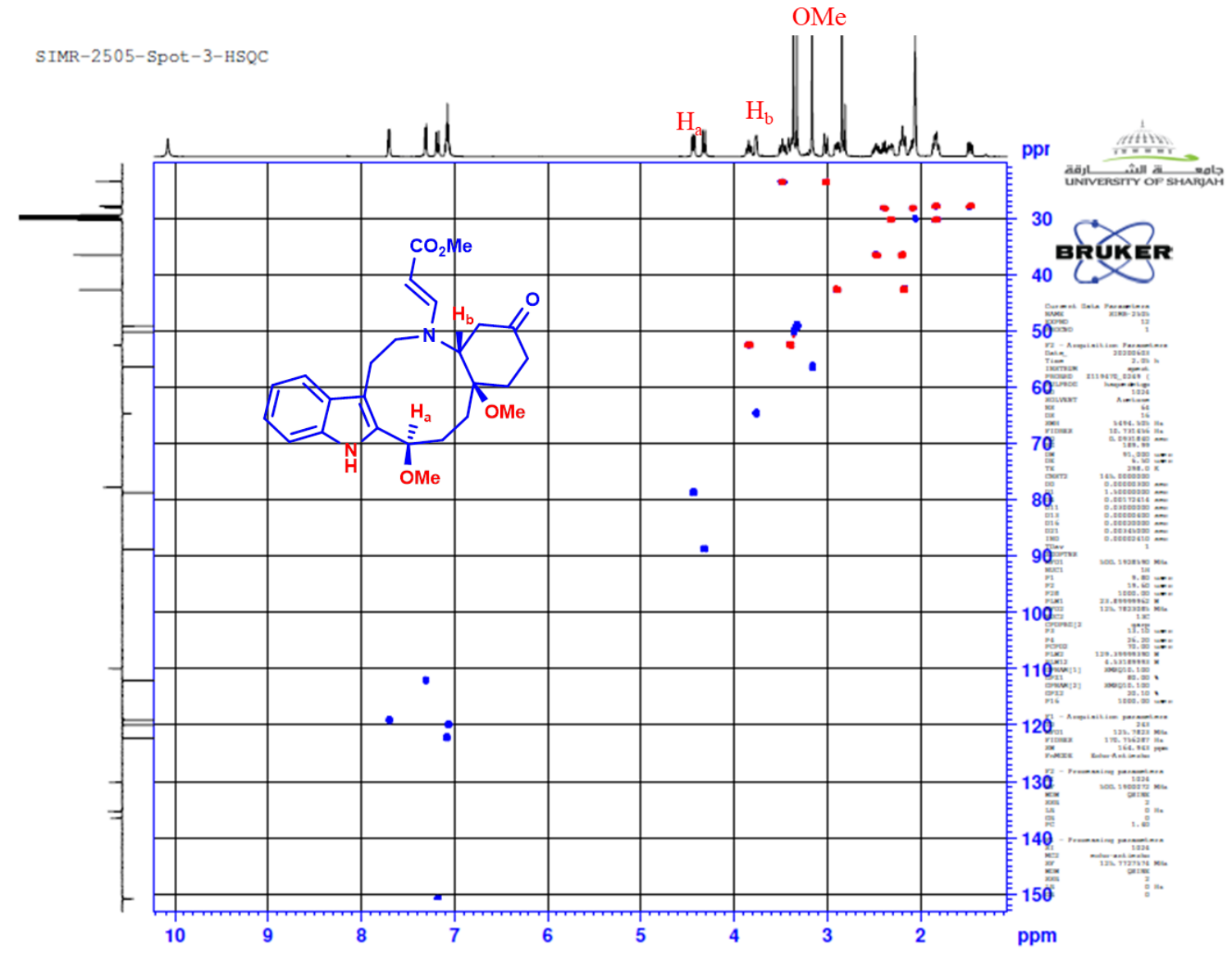

Figure S95 HSQC NMR (500 MHz, Acetone-d $\left.)_{6}\right)$ spectrum of methyl (E)-3-((4aR,13R,15aR)13,15a-dimethoxy-3-oxo-1,2,3,4,4a,6,7,12,13,14,15,15a-dodecahydro-5Hbenzo[9,10]azecino[5,4-b]indol-5-yl)acrylate (25a) 


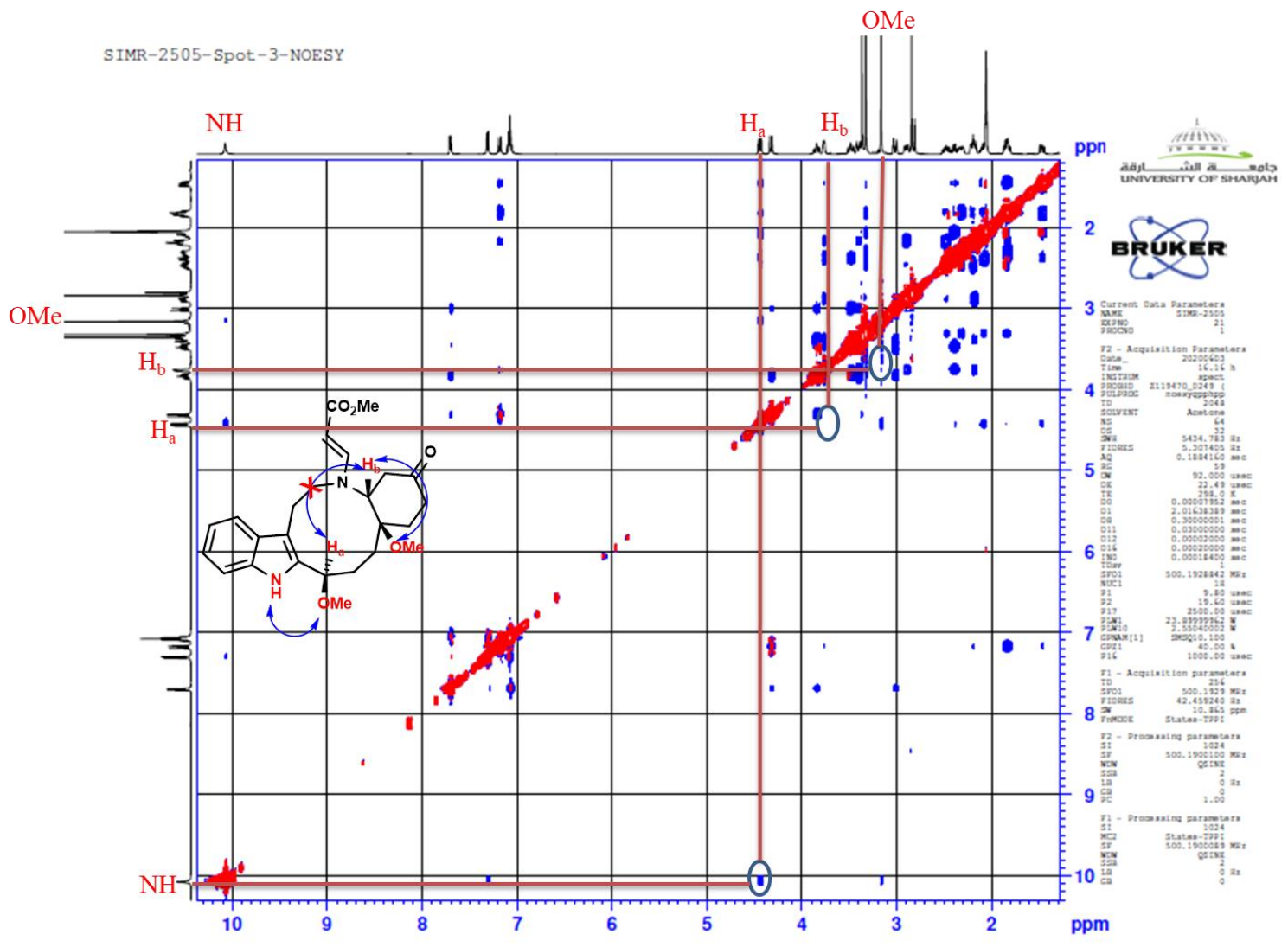

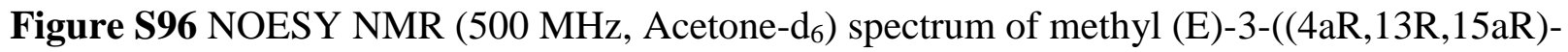
13,15a-dimethoxy-3-oxo-1,2,3,4,4a,6,7,12,13,14,15,15a-dodecahydro-5Hbenzo[9,10]azecino[5,4-b]indol-5-yl)acrylate (25a) 


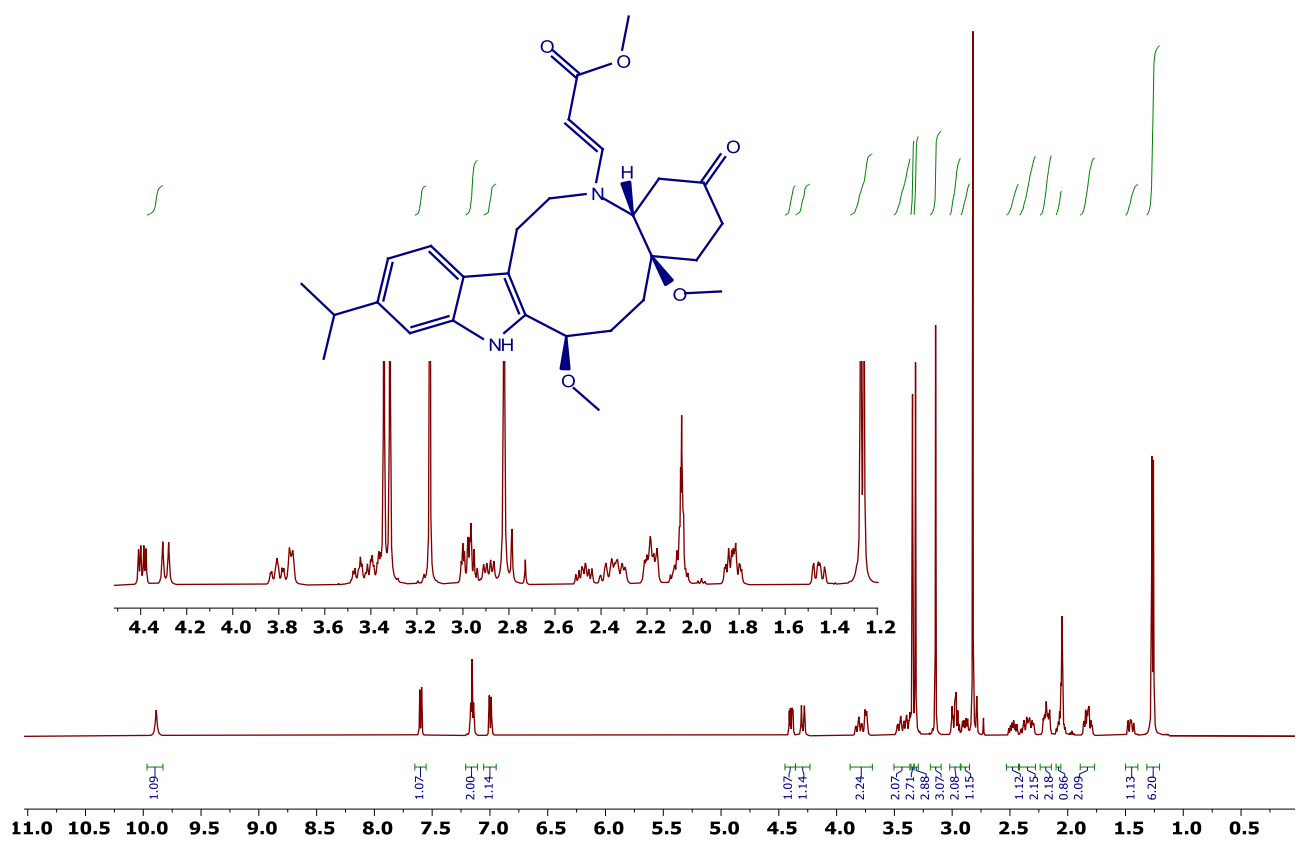

Figure S97 ${ }^{1} \mathrm{H}$ NMR (500 MHz, Acetone-d $)$ spectrum of methyl (E)-3-((4aR,13R,15aR)-10isopropyl-13,15a-dimethoxy-3-oxo-1,2,3,4,4a,6,7,12,13,14,15,15a-dodecahydro-5Hbenzo[9,10]azecino[5,4-b]indol-5-yl)acrylate $(\mathbf{2 5 b})$

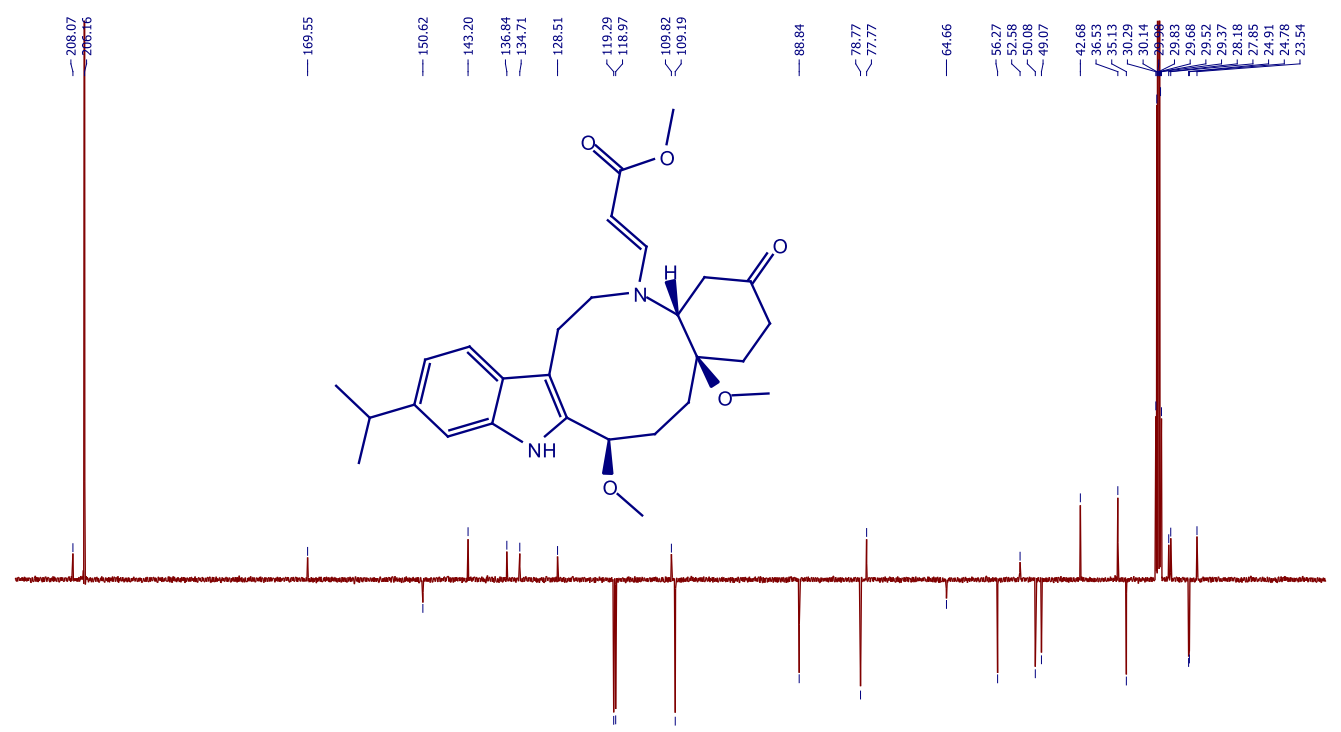

$\begin{array}{llllllllllllllllll}210 & 190 & 170 & 150 & 130 & 110 & 90 & 80 & 70 & 60 & 50 & 40 & 30 & 20 & 10\end{array}$

Figure S98 ${ }^{13} \mathrm{C}\left\{{ }^{1} \mathrm{H}\right\}$ APT NMR (125 MHz, Acetone-d 6 ) spectrum of methyl (E)-3((4aR,13R,15aR)-10-isopropyl-13,15a-dimethoxy-3-oxo-1,2,3,4,4a,6,7,12,13,14,15,15adodecahydro-5H-benzo[9,10]azecino[5,4-b]indol-5-yl)acrylate (25b) 


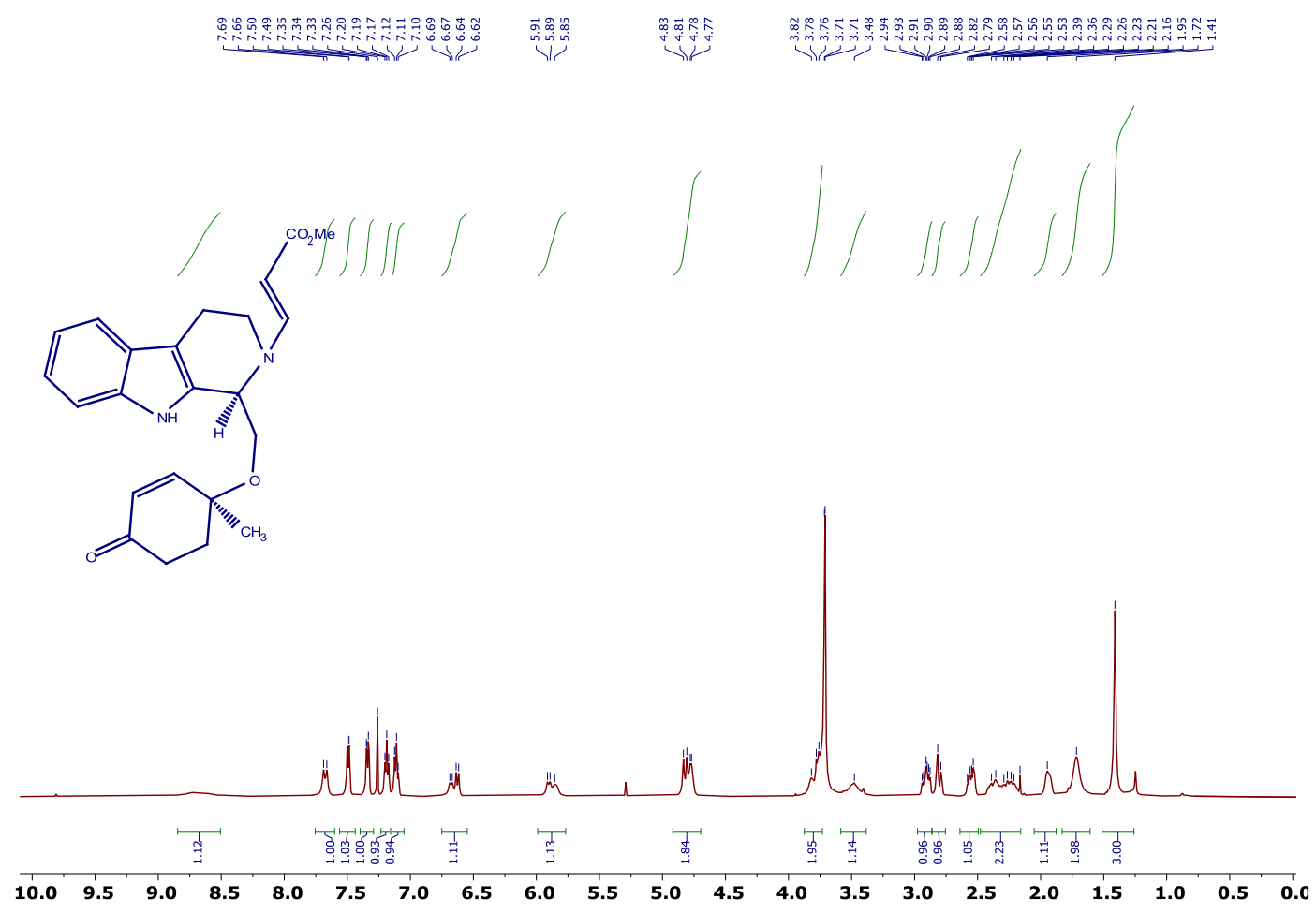

Figure S99 ${ }^{1} \mathrm{H}$ NMR $\left(500 \mathrm{MHz}, \mathrm{CDCl}_{3}\right)$ spectrum of methyl (E)-4-((R)-1-((((S)-1-methyl-4oxocyclohex-2-en-1-yl)oxy)methyl)-1,3,4,9-tetrahydro-2H-pyrido[3,4-b]indol-2-yl)but-2-enoate (27)

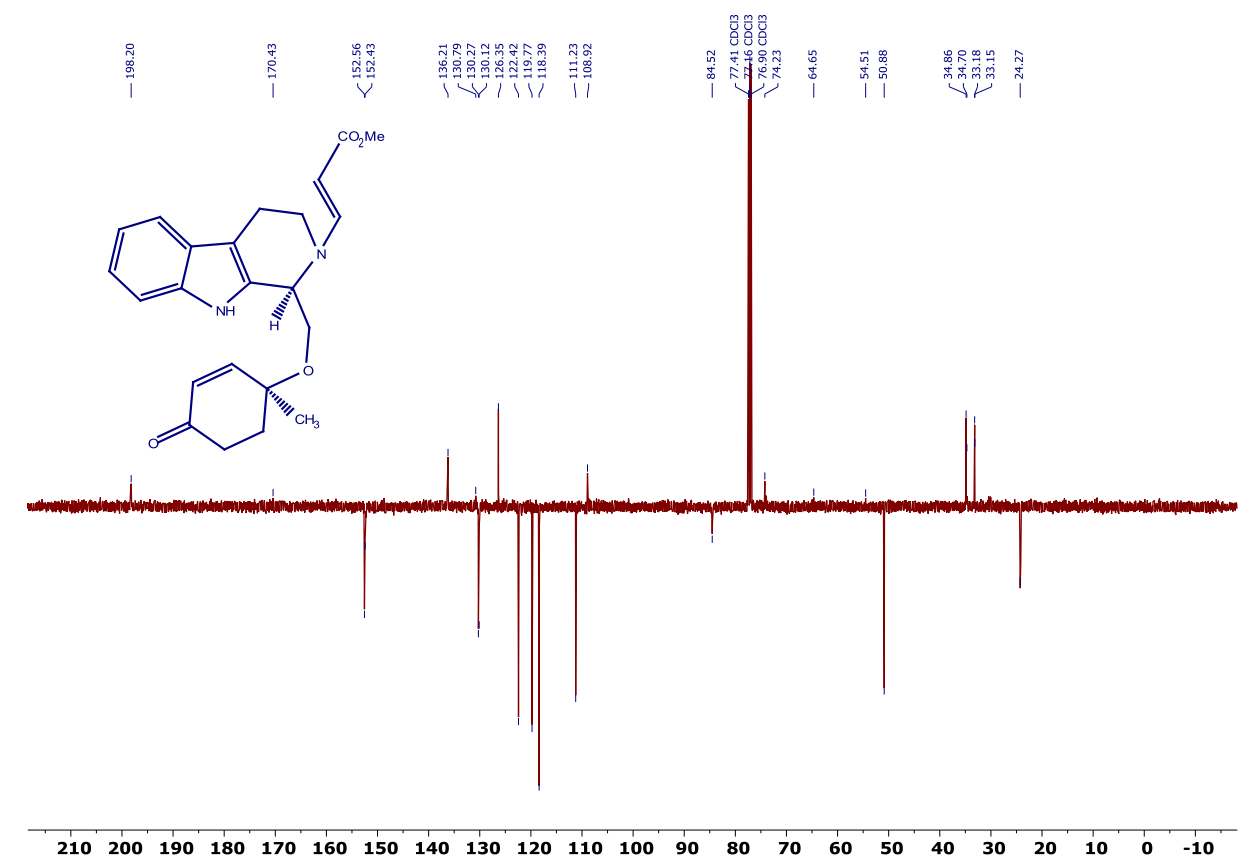

Figure S100 ${ }^{13} \mathrm{C}\left\{{ }^{1} \mathrm{H}\right\}$ APT NMR $\left(125 \mathrm{MHz}, \mathrm{CDCl}_{3}\right)$ spectrum of methyl (E)-4-((R)-1-((((S)-1methyl-4-oxocyclohex-2-en-1-yl)oxy)methyl)-1,3,4,9-tetrahydro-2H-pyrido[3,4-b]indol-2yl)but-2-enoate (27) 


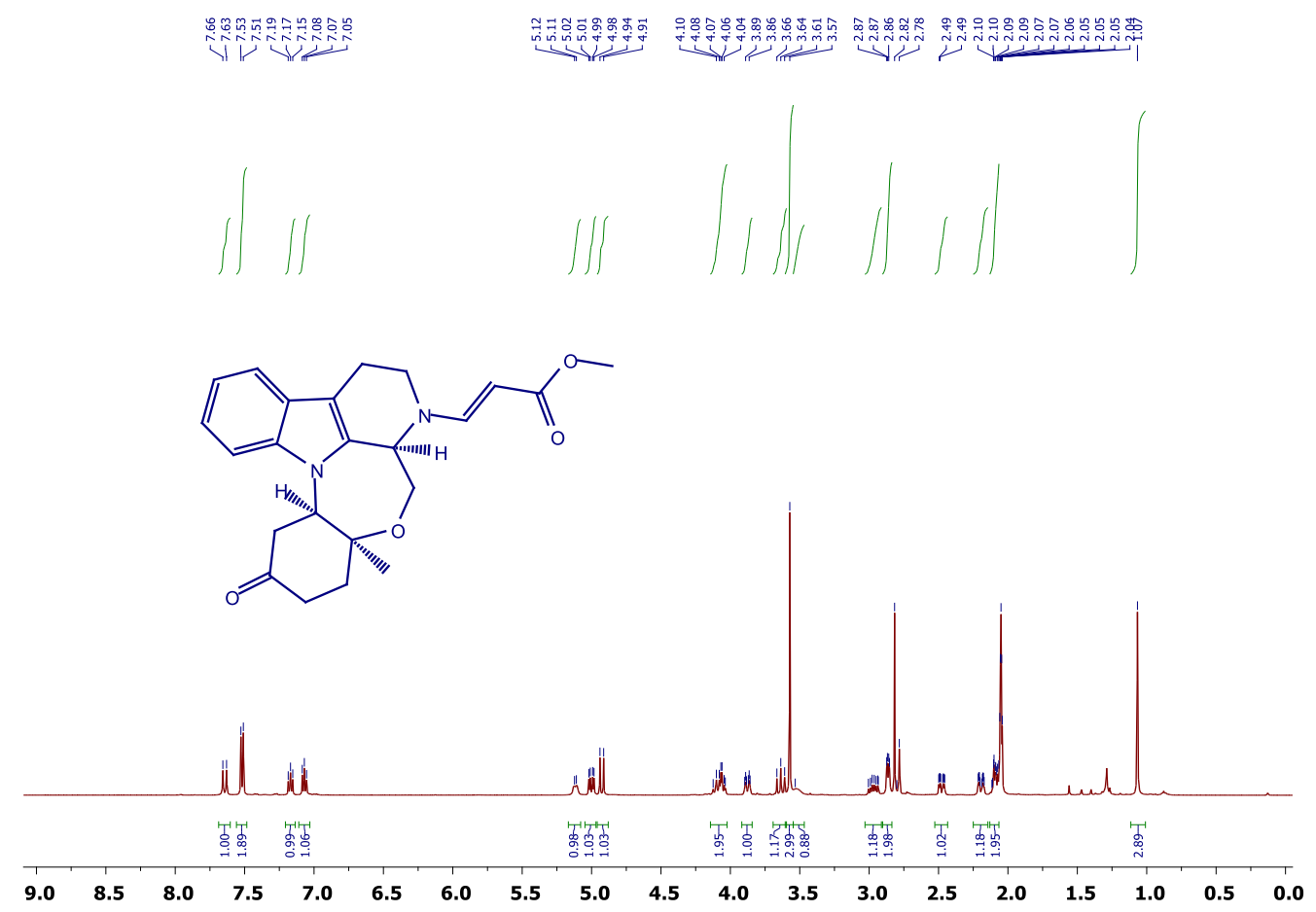

Figure S101 ${ }^{1} \mathrm{H}$ NMR (500 MHz, acetone- $\left.\mathrm{d}_{6}\right)$ spectrum of methyl (E)-3-((3aR,5aS,9aR)-5amethyl-8-oxo-1,2,3a,4,5a,6,7,8,9,9a-decahydro-3H-5-oxa-3,9b-diazabenzo[4,5]cyclohepta[1,2,3jk]fluoren-3-yl)acrylate (28)
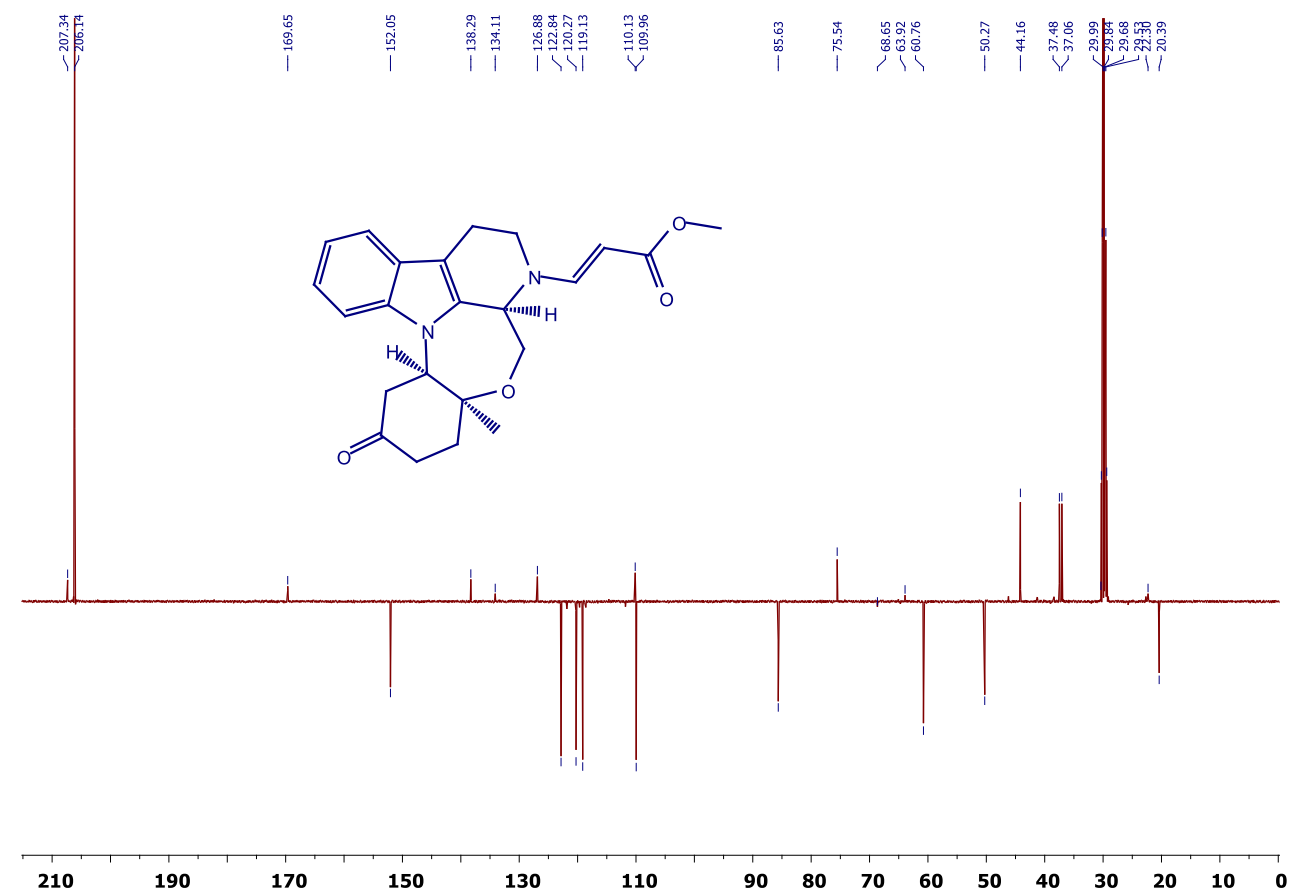

Figure S102 ${ }^{13} \mathrm{C}\left\{{ }^{1} \mathrm{H}\right\}$ APT NMR (125 MHz, acetone-d $\left.\mathrm{d}_{6}\right)$ spectrum of methyl (E)-3((3aR,5aS,9aR)-5a-methyl-8-oxo-1,2,3a,4,5a,6,7,8,9,9a-decahydro-3H-5-oxa-3,9bdiazabenzo[4,5]cyclohepta[1,2,3-jk]fluoren-3-yl)acrylate (28) 


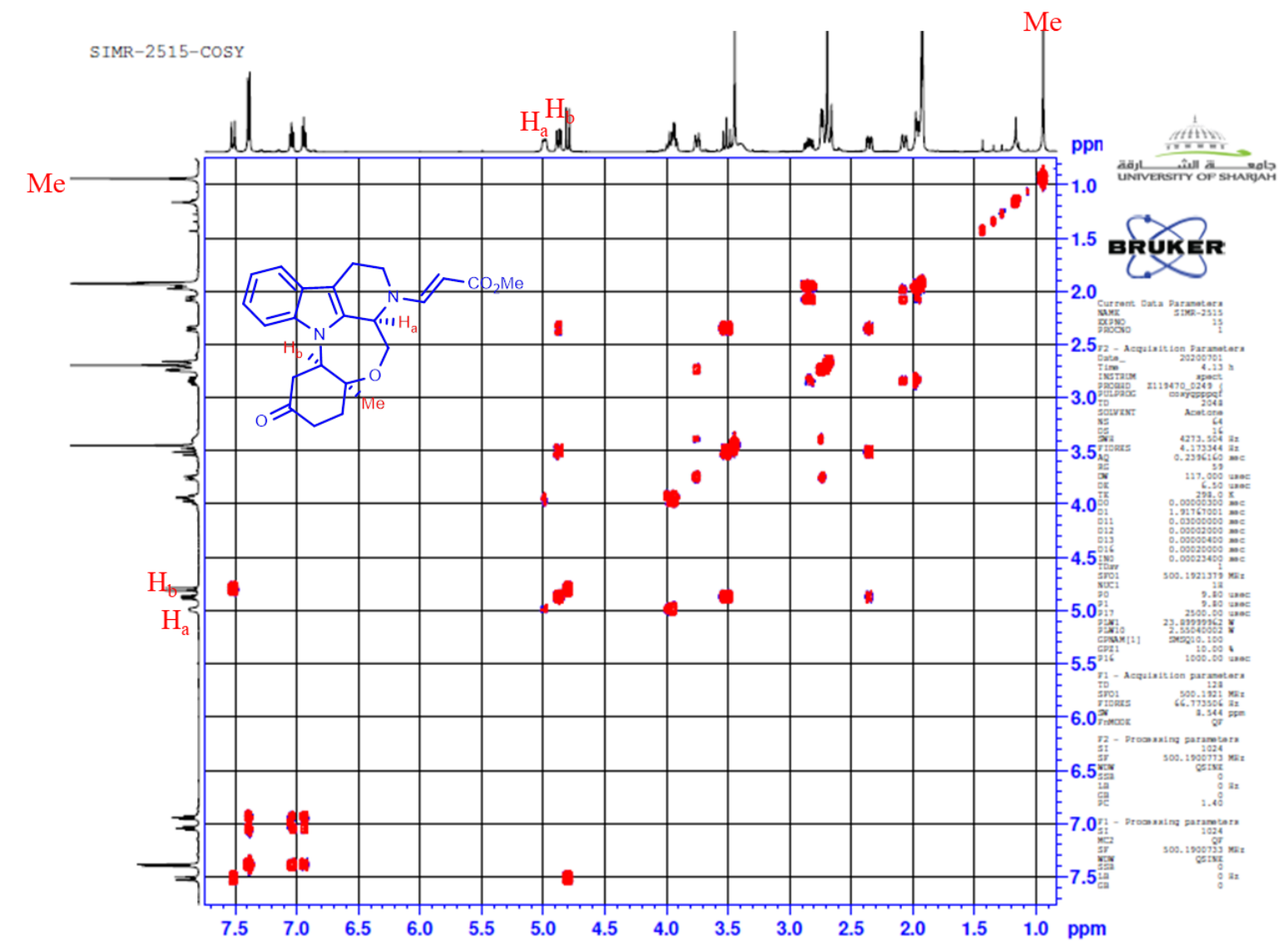

Figure S103 COSY NMR (500 MHz, acetone-d $\left.)_{6}\right)$ spectrum of methyl (E)-3-((3aR,5aS,9aR)-5amethyl-8-oxo-1,2,3a,4,5a,6,7,8,9,9a-decahydro-3H-5-oxa-3,9b-diazabenzo[4,5]cyclohepta[1,2,3jk]fluoren-3-yl)acrylate (28) 


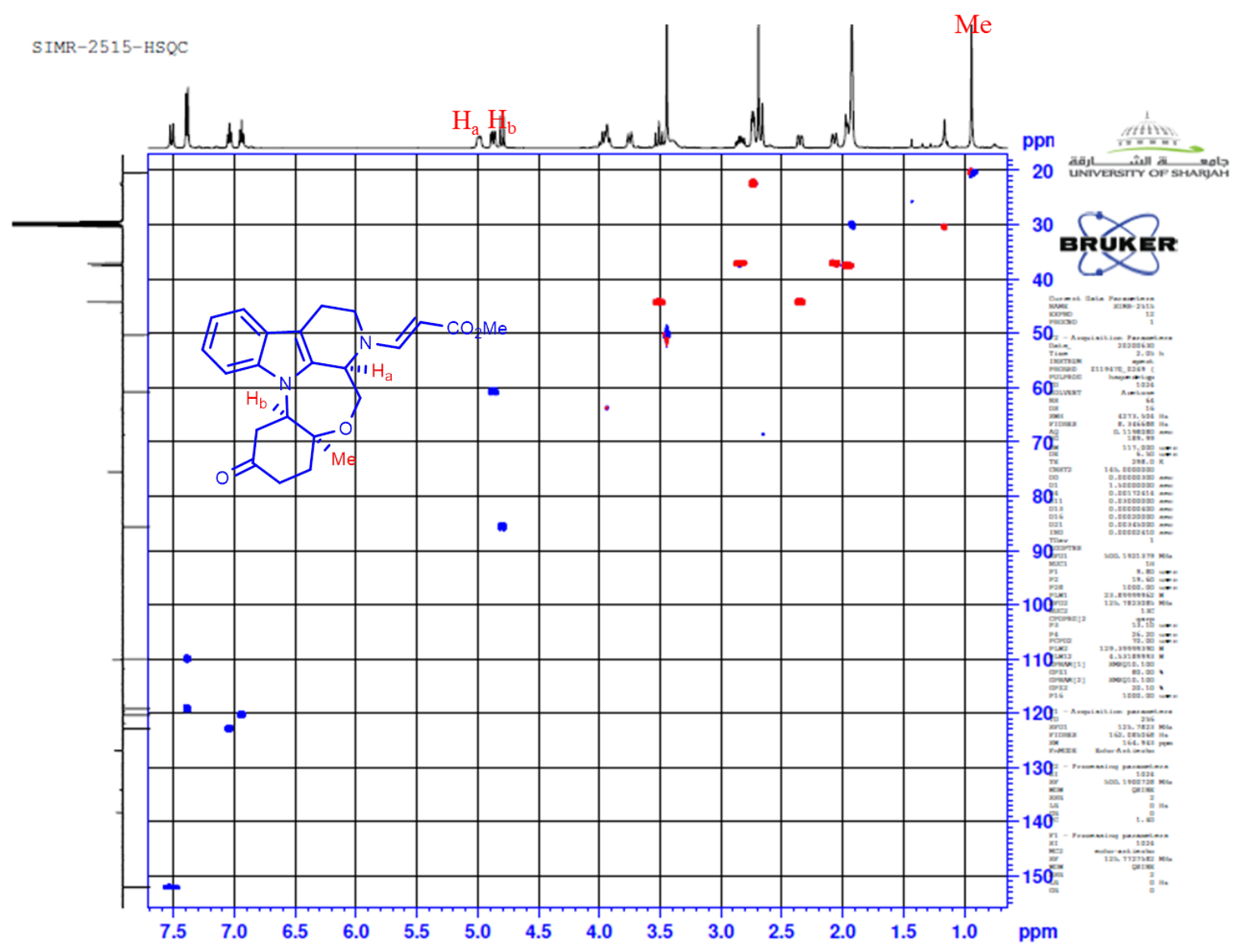

Figure S104 HSQC NMR (500 MHz, Acetone-d $\left.\mathrm{d}_{6}\right)$ spectrum of methyl (E)-3-((3aR,5aS,9aR)-5amethyl-8-oxo-1,2,3a,4,5a,6,7,8,9,9a-decahydro-3H-5-oxa-3,9b-diazabenzo[4,5]cyclohepta[1,2,3jk]fluoren-3-yl)acrylate (28) 


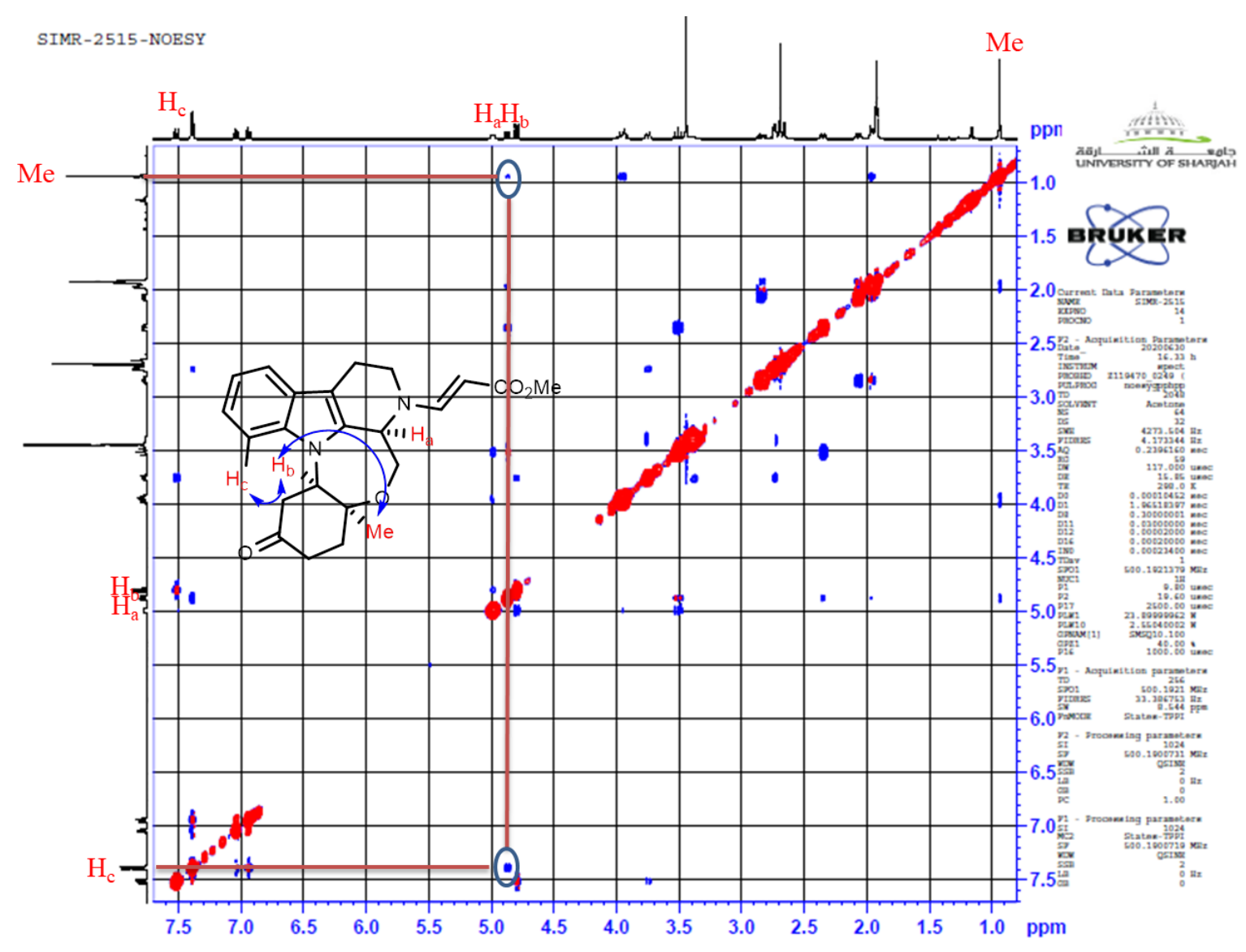

Figure S105 NOESY NMR (500 MHz, Acetone-d $)_{6}$ spectrum of methyl (E)-3-((3aR,5aS,9aR)5a-methyl-8-oxo-1,2,3a,4,5a,6,7,8,9,9a-decahydro-3H-5-oxa-3,9bdiazabenzo[4,5]cyclohepta[1,2,3-jk]fluoren-3-yl)acrylate (28) 


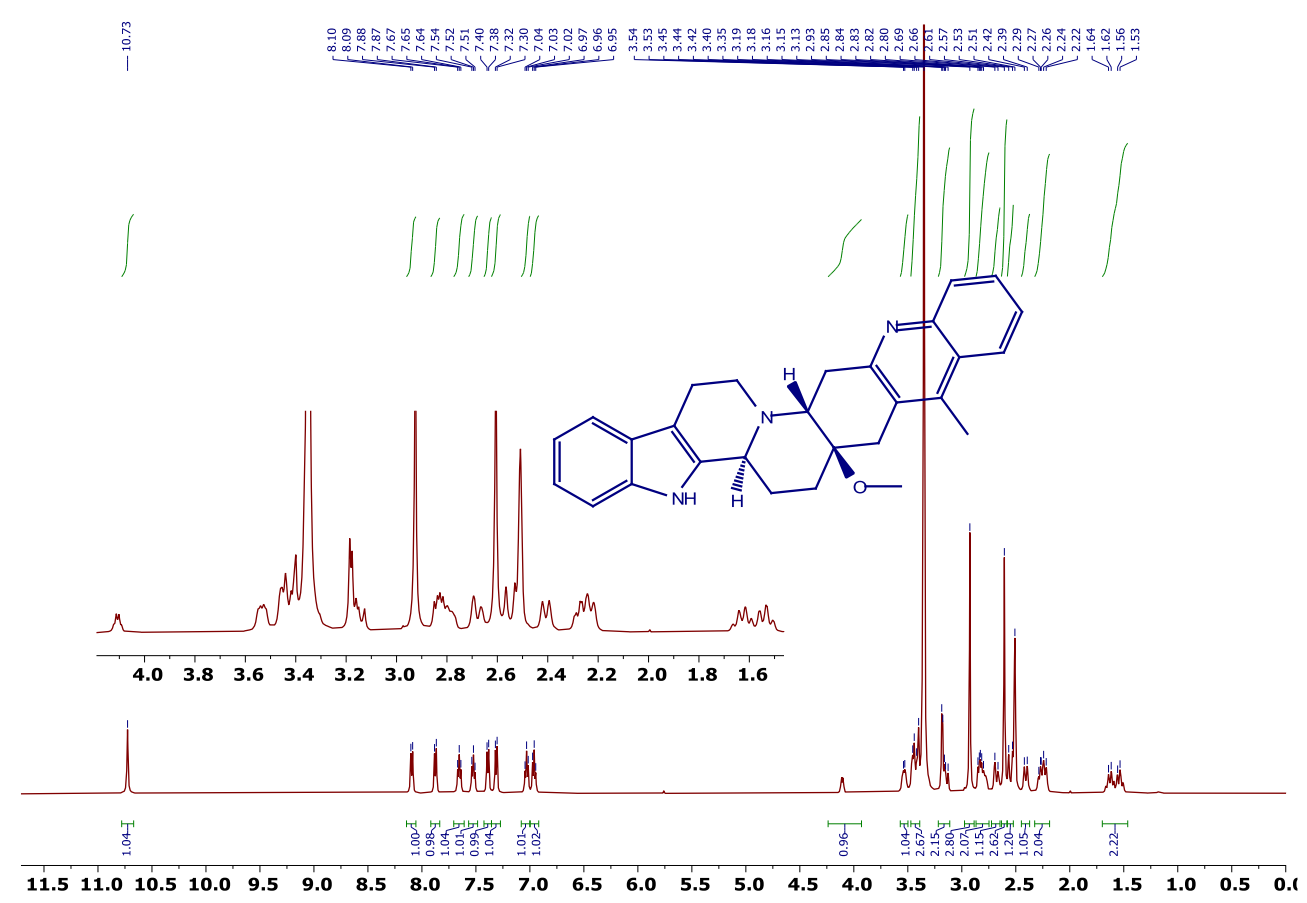

Figure S106 ${ }^{1} \mathrm{H}$ NMR ( $500 \mathrm{MHz}$, DMSO-d $\mathrm{d}_{6}$ ) spectrum of (5bS,7aR,15aR)-7a-methoxy-9methyl-5,5b,6,7,7a,8,15,15a,17,18-decahydroindolo[3',2':8,9]quinolizino[3,4-b]acridine (30a)
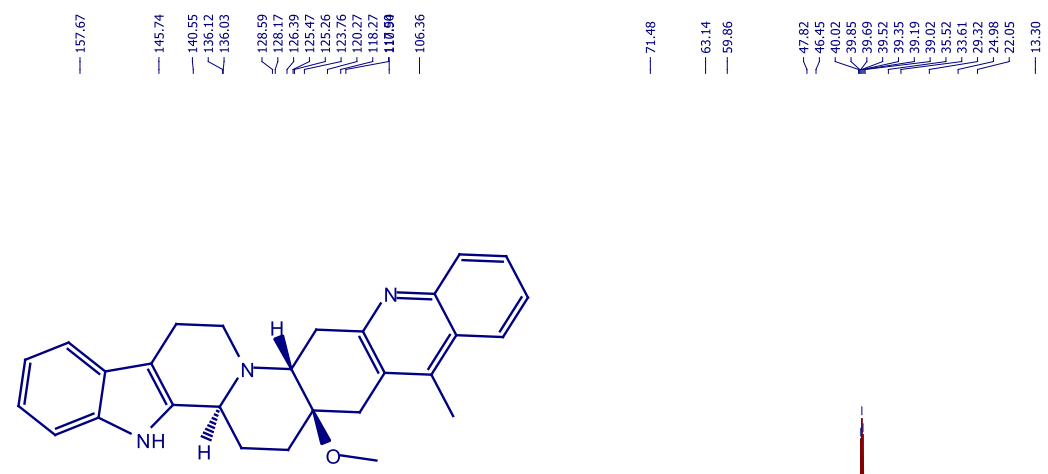

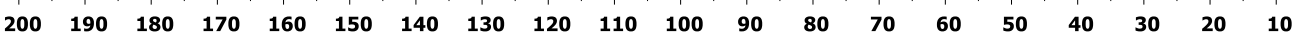

Figure S107 ${ }^{13} \mathrm{C}\left\{{ }^{1} \mathrm{H}\right\}$ APT NMR (125 MHz, DMSO-d 6 ) spectrum of (5bS,7aR,15aR)-7amethoxy-9-methyl-5,5b,6,7,7a,8,15,15a,17,18-decahydroindolo[3',2':8,9]quinolizino[3,4b]acridine (30a) 


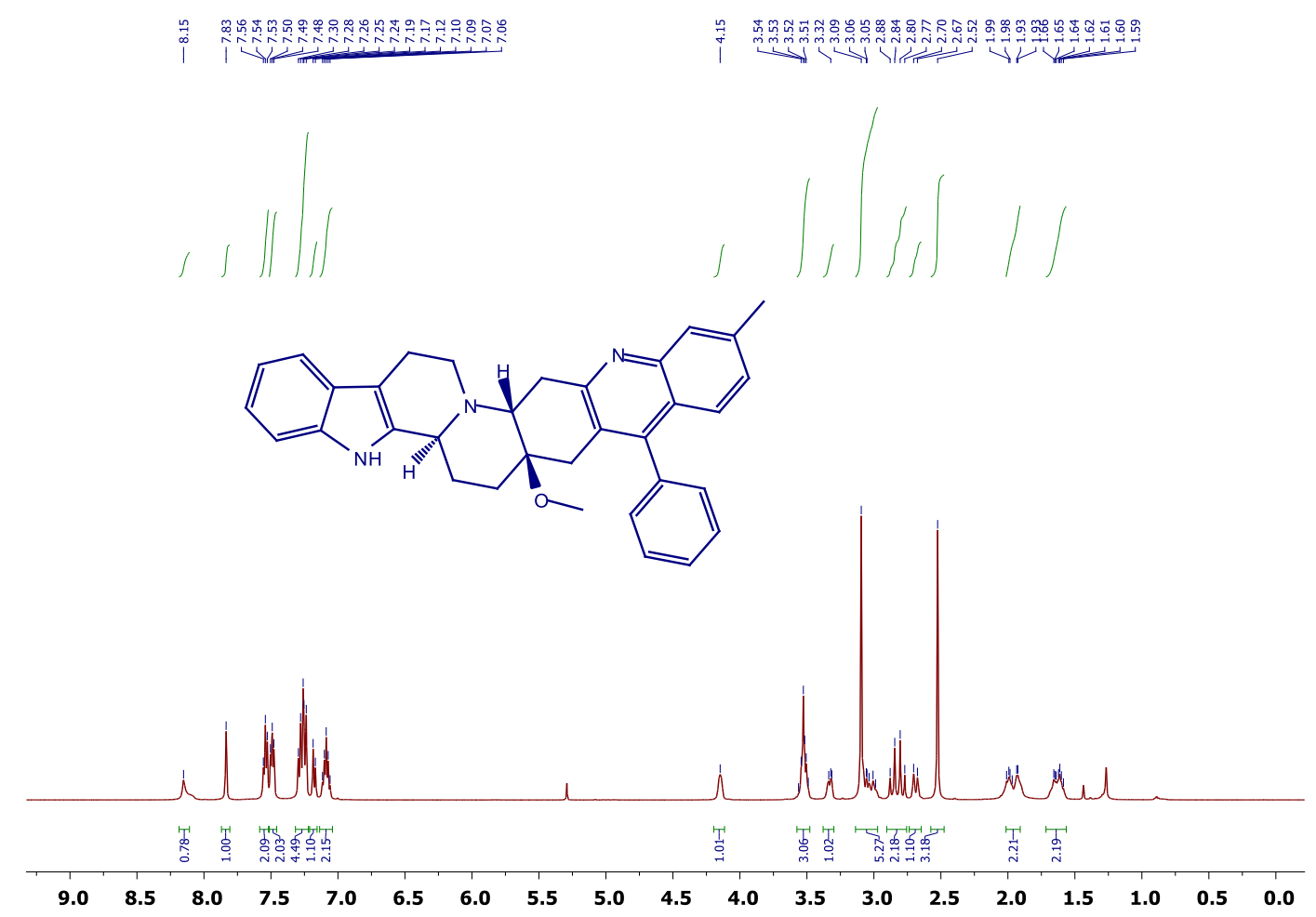

Figure S108 ${ }^{1} \mathrm{H}$ NMR $\left(500 \mathrm{MHz}, \mathrm{CDCl}_{3}\right.$ ) spectrum of (5bS,7aR,15aR)-7a-methoxy-12-methyl9-phenyl-5,5b,6,7,7a,8,15,15a, 17,18-decahydroindolo[3',2':8,9]quinolizino[3,4-b]acridine (30b)

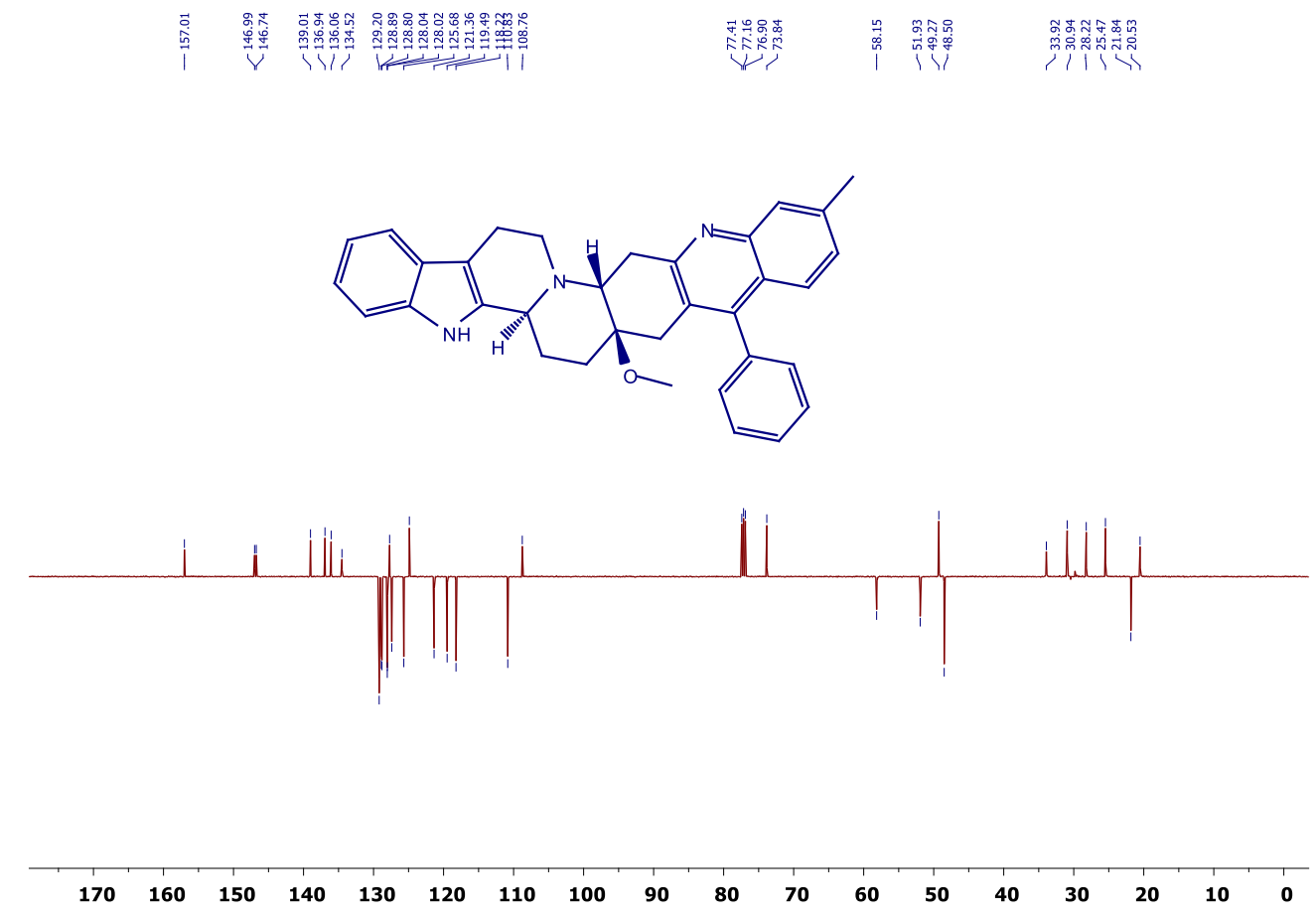

Figure S109 ${ }^{13} \mathrm{C}\left\{{ }^{1} \mathrm{H}\right\}$ APT NMR $\left(125 \mathrm{MHz}, \mathrm{CDCl}_{3}\right)$ spectrum of (5bS,7aR,15aR)-7a-methoxy12-methyl-9-phenyl-5,5b,6,7,7a, , , 15,15a, 17,18-decahydroindolo[3',2':8,9]quinolizino[3,4b]acridine (30b) 


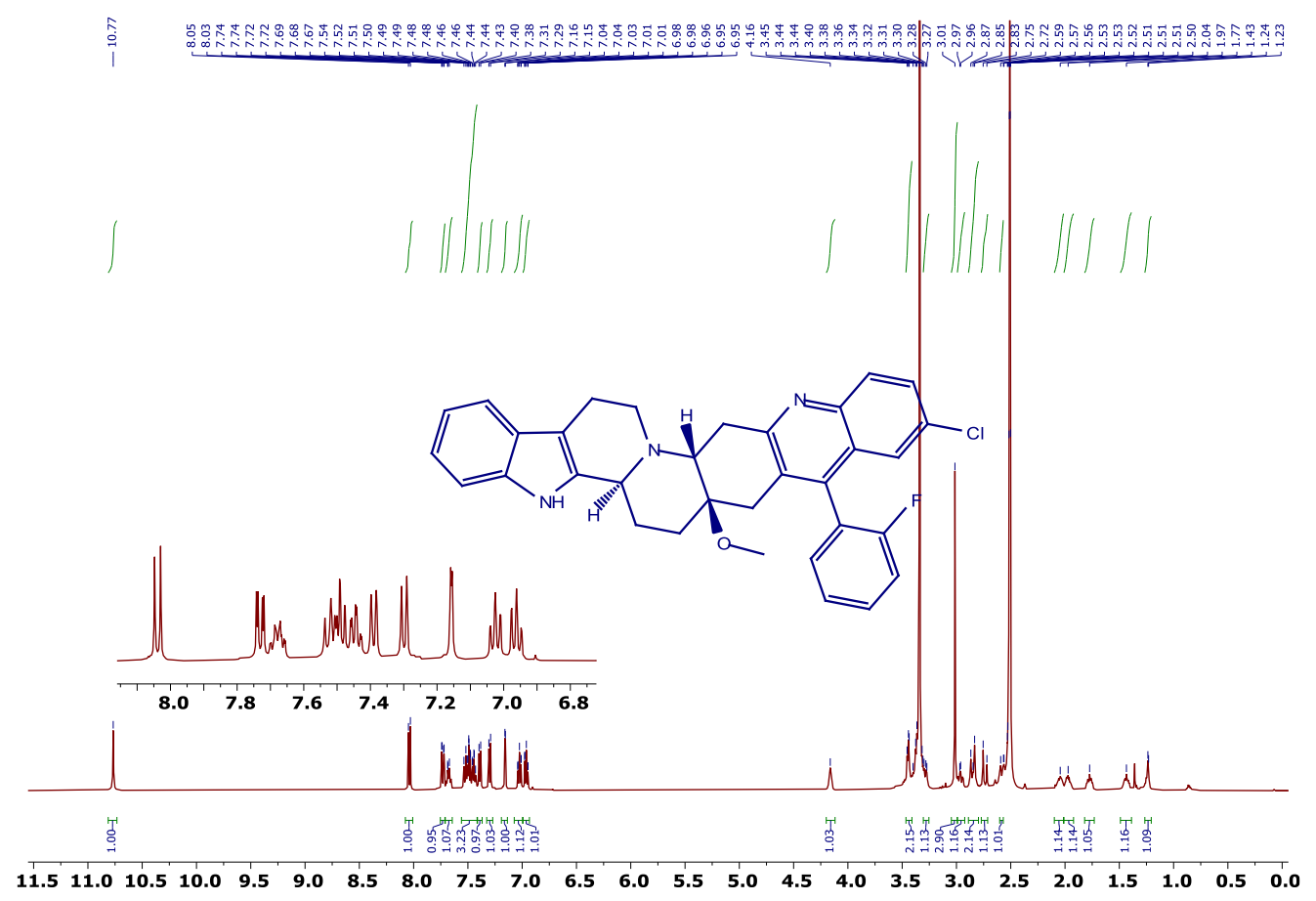

Figure S110 ${ }^{1} \mathrm{H}$ NMR (500 MHz, DMSO-d 6 ) spectrum of (5bS,7aR,15aR)-11-chloro-9-(2fluorophenyl)-7a-methoxy-5,5b,6,7,7a,8,15,15a,17,18-decahydroindolo[3',2':8,9]quinolizino[3,4b]acridine (30c)

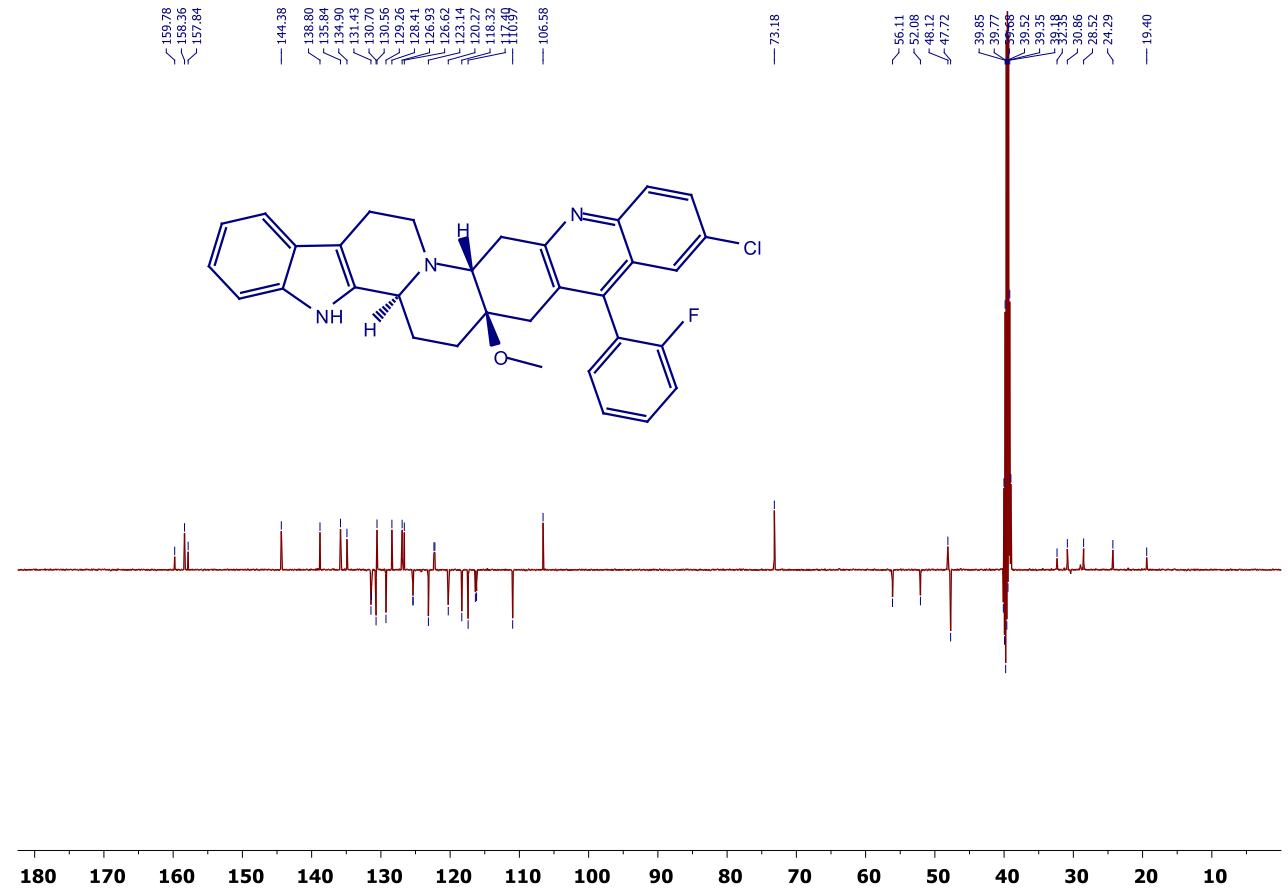

Figure S111 ${ }^{13} \mathrm{C}\left\{{ }^{1} \mathrm{H}\right\}$ APT NMR (125 MHz, DMSO-d $\left.{ }_{6}\right)$ spectrum of (5bS,7aR,15aR)-11-chloro9-(2-fluorophenyl)-7a-methoxy-5,5b,6,7,7a,8,15,15a,17,18decahydroindolo[3',2':8,9]quinolizino[3,4-b]acridine (30c) 


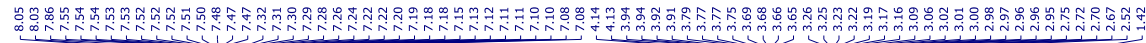

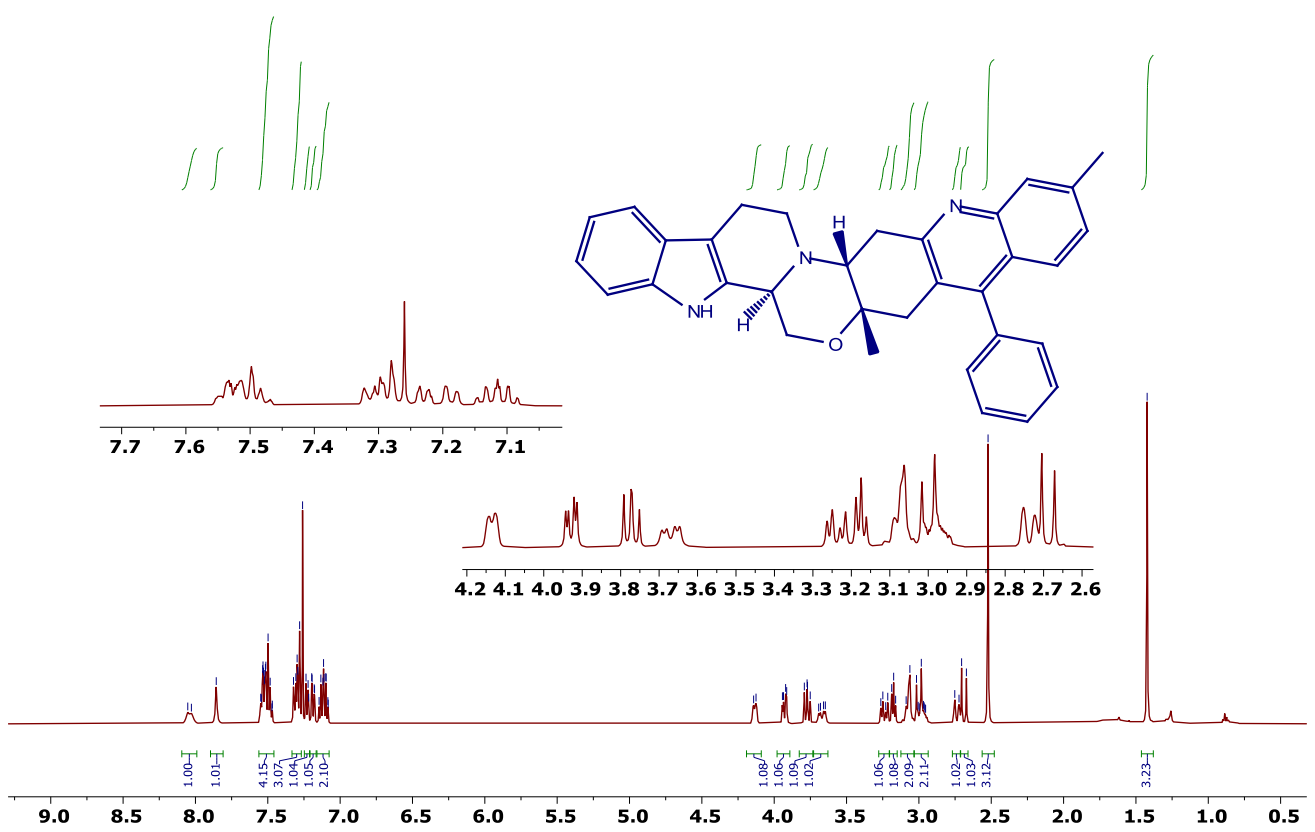

Figure S112 ${ }^{1} \mathrm{H}$ NMR (500 MHz, $\mathrm{CDCl}_{3}$ ) spectrum of (5bR,7aS,15aR)-7a, 12-dimethyl-9phenyl-5b,6,7a,8,15,15a,17,18-octahydro-5H-indolo[2",3": 3',4']pyrido[1',2':4,5][1,4]oxazino[2,3b]acridine (31a)
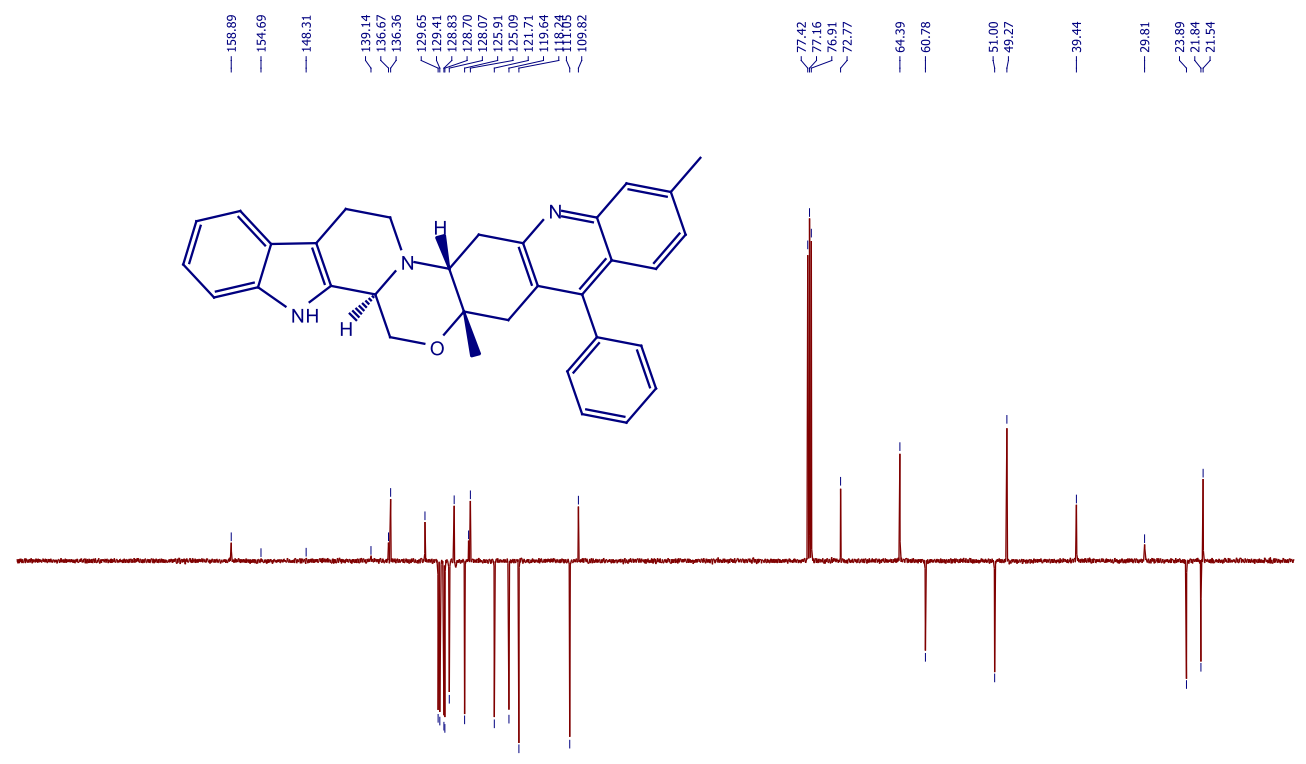

$\begin{array}{lllllllllllllllllll}180 & 170 & 160 & 150 & 140 & 130 & 120 & 110 & 100 & 90 & 80 & 70 & 60 & 50 & 40 & 30 & 20 & 10\end{array}$

Figure S113 ${ }^{13} \mathrm{C}\left\{{ }^{1} \mathrm{H}\right\}$ APT NMR $\left(125 \mathrm{MHz}, \mathrm{CDCl}_{3}\right)$ spectrum of $(5 \mathrm{bR}, 7 \mathrm{aS}, 15 \mathrm{aR})-7 \mathrm{a}, 12-$ dimethyl-9-phenyl-5b,6,7a,8,15,15a,17,18-octahydro-5Hindolo[2",3":3',4']pyrido[1',2':4,5][1,4]oxazino[2,3-b]acridine (31a) 


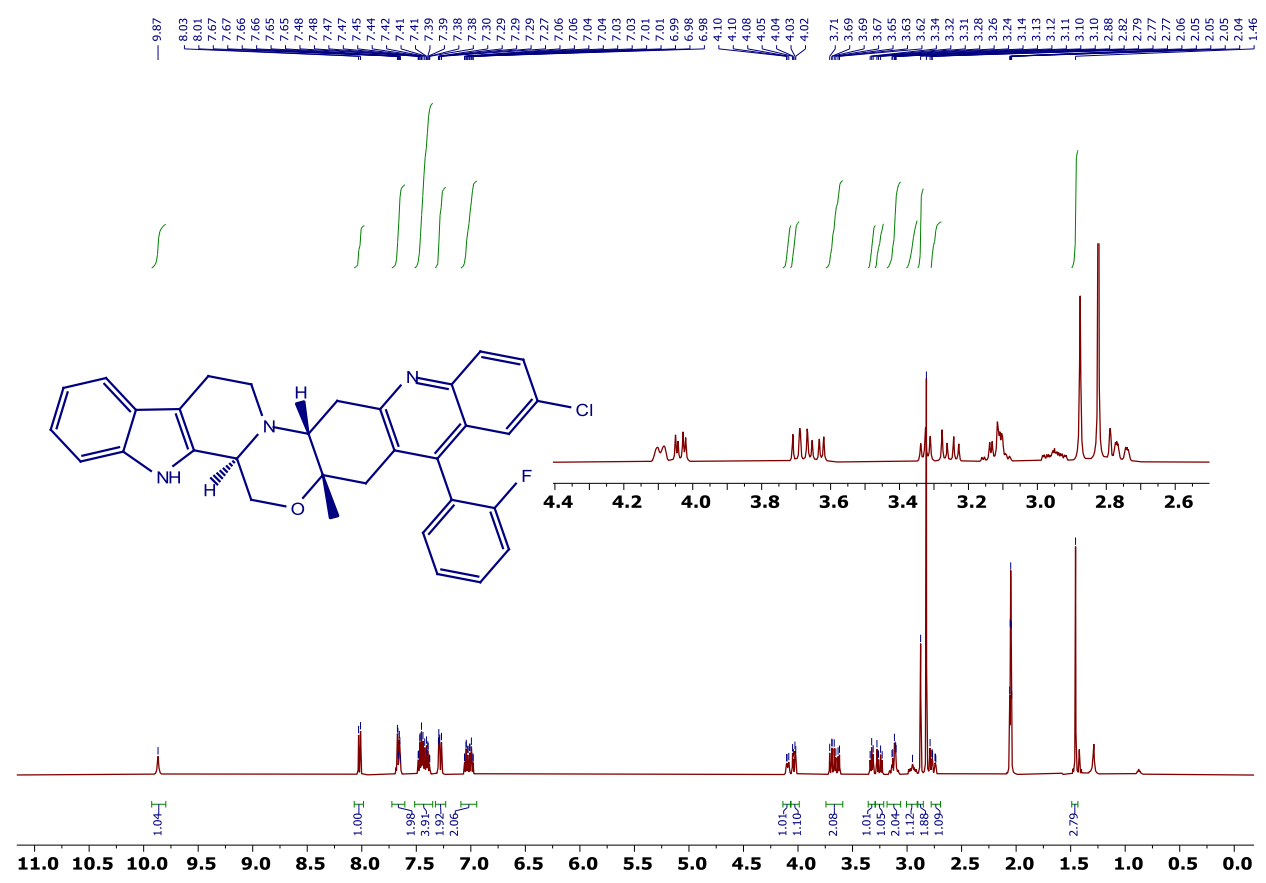

Figure S114 ${ }^{1} \mathrm{H}$ NMR $(500 \mathrm{MHz}$, Acetone-d 6 ) spectrum of (5bR,7aS,15aR)-11-chloro-9-(2fluorophenyl)-7a-methyl-5b,6,7a,8,15,15a,17,18-octahydro-5Hindolo[2", 3":3',4']pyrido[1',2':4,5][1,4] oxazino[2,3-b]acridine (31b)

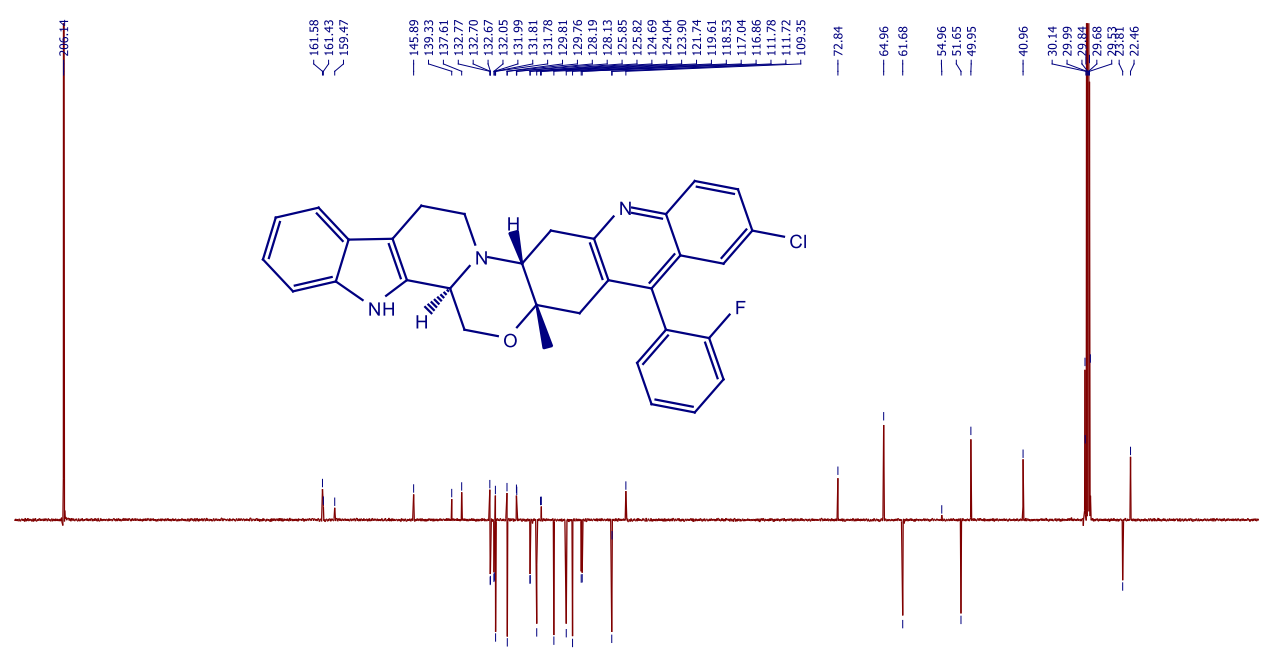

\begin{tabular}{llllllllllllllll}
\hline 210 & 190 & 170 & 150 & 130 & 110 & 90 & 80 & 70 & 60 & 50 & 40 & 30 & 20 & 10
\end{tabular}

Figure S115 ${ }^{13} \mathrm{C}\left\{{ }^{1} \mathrm{H}\right\}$ APT NMR (125 MHz, Acetone-d $\left.\mathrm{d}_{6}\right)$ spectrum of (5bR,7aS, 15aR)-11-chloro9-(2-fluorophenyl)-7a-methyl-5b,6,7a,8,15,15a,17,18-octahydro-5Hindolo[2", 3": 3',4']pyrido[1',2':4,5][1,4] oxazino[2,3-b]acridine (31b) 


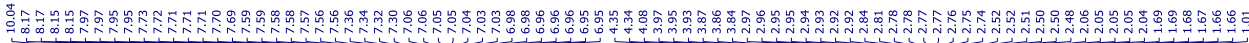
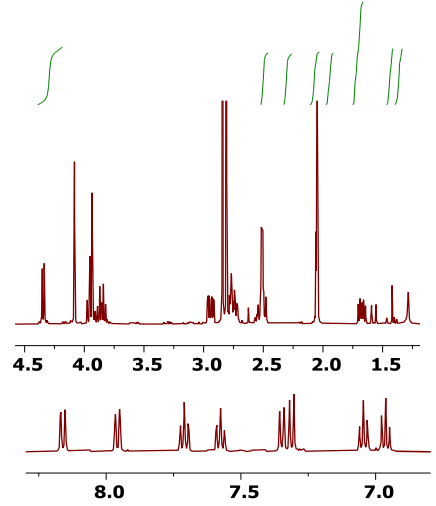
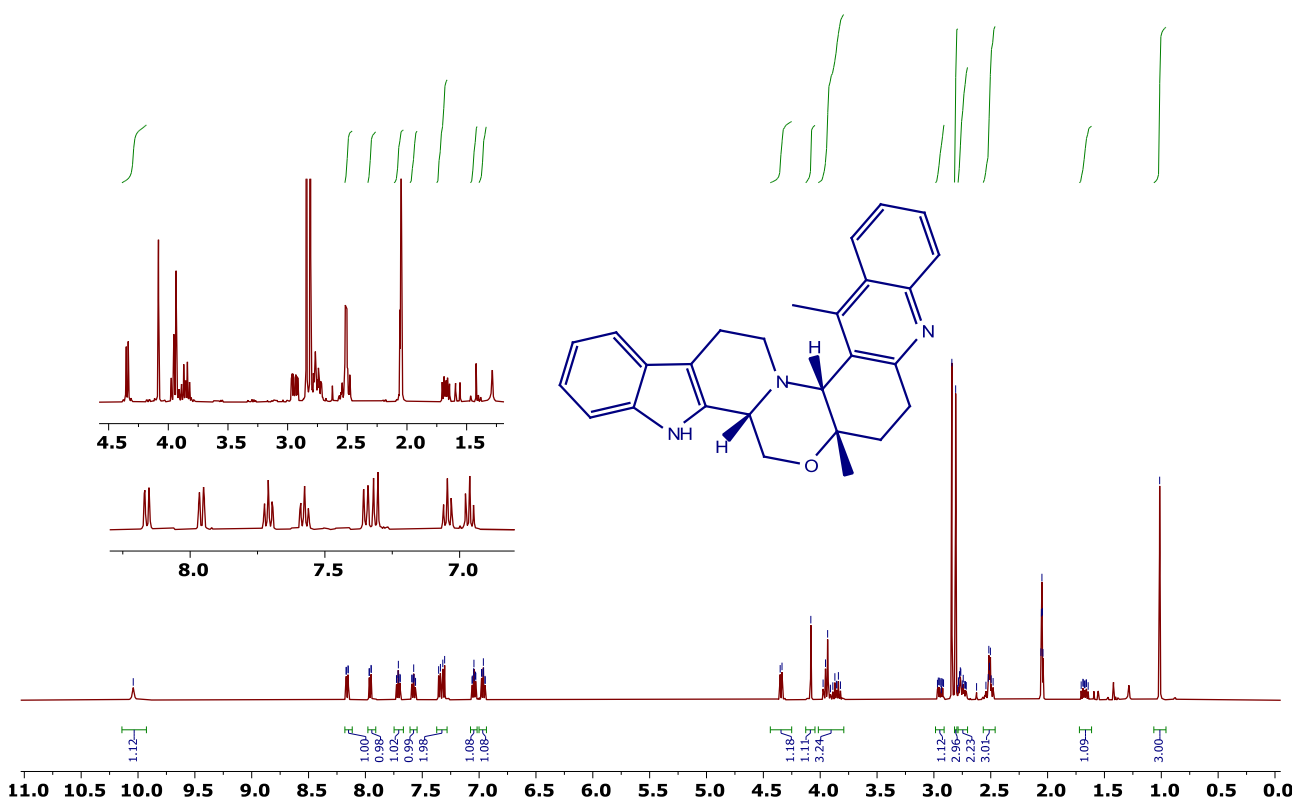

Figure S116 ${ }^{1} \mathrm{H}$ NMR $\left(500 \mathrm{MHz}\right.$, Acetone-d $\left.{ }_{6}\right)$ spectrum of (5bS,7aS,15bR)-7a,15-dimethyl5b,6,7a,8,9,15b,17,18-octahydro-5H-indolo[2",3": 3',4']pyrido[1',2':4,5][1,4]oxazino[3,2a]acridine (33a)
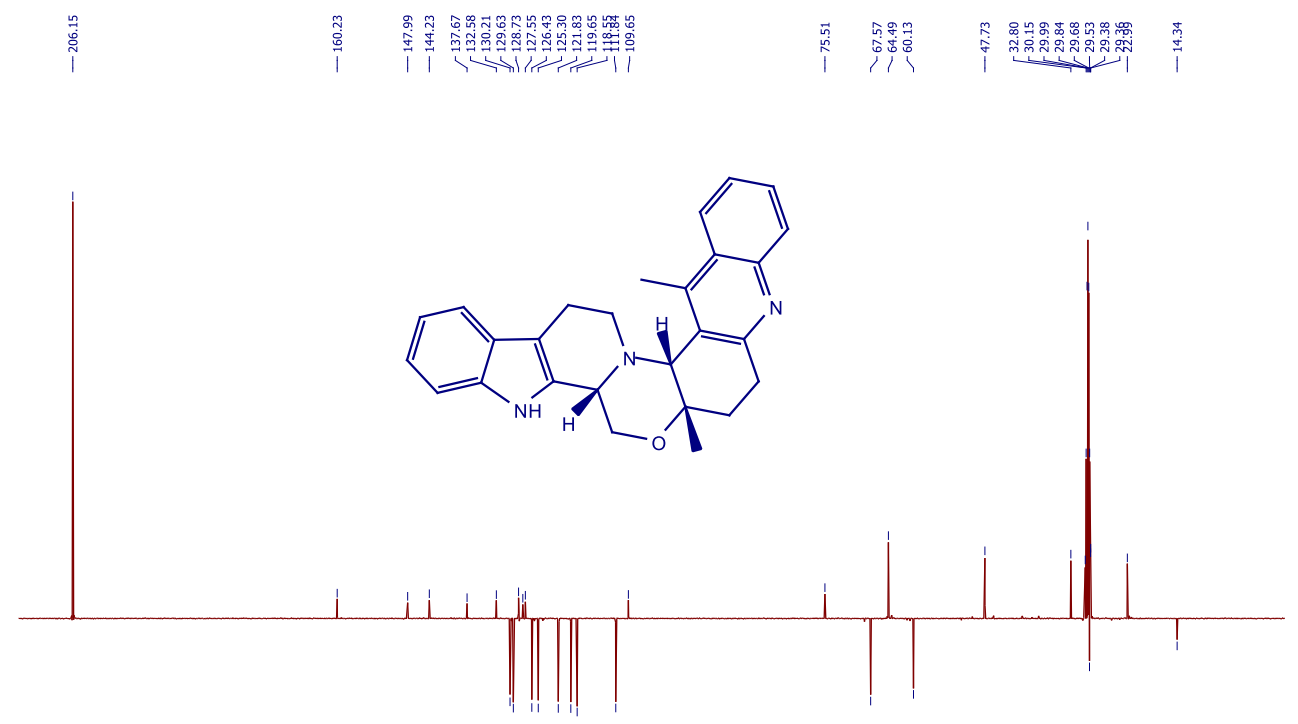

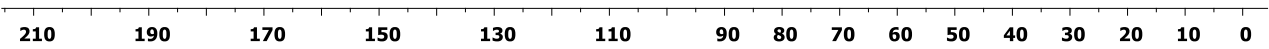

Figure S117 ${ }^{13} \mathrm{C}\left\{{ }^{1} \mathrm{H}\right\}$ APT NMR (125 MHz, Acetone-d $\left.{ }_{6}\right)$ spectrum of (5bS,7aS, 15bR)-7a,15dimethyl-5b,6,7a,8,9,15b,17,18-octahydro-5Hindolo[2",3":3',4']pyrido[1',2':4,5][1,4]oxazino[3,2-a]acridine (33a) 


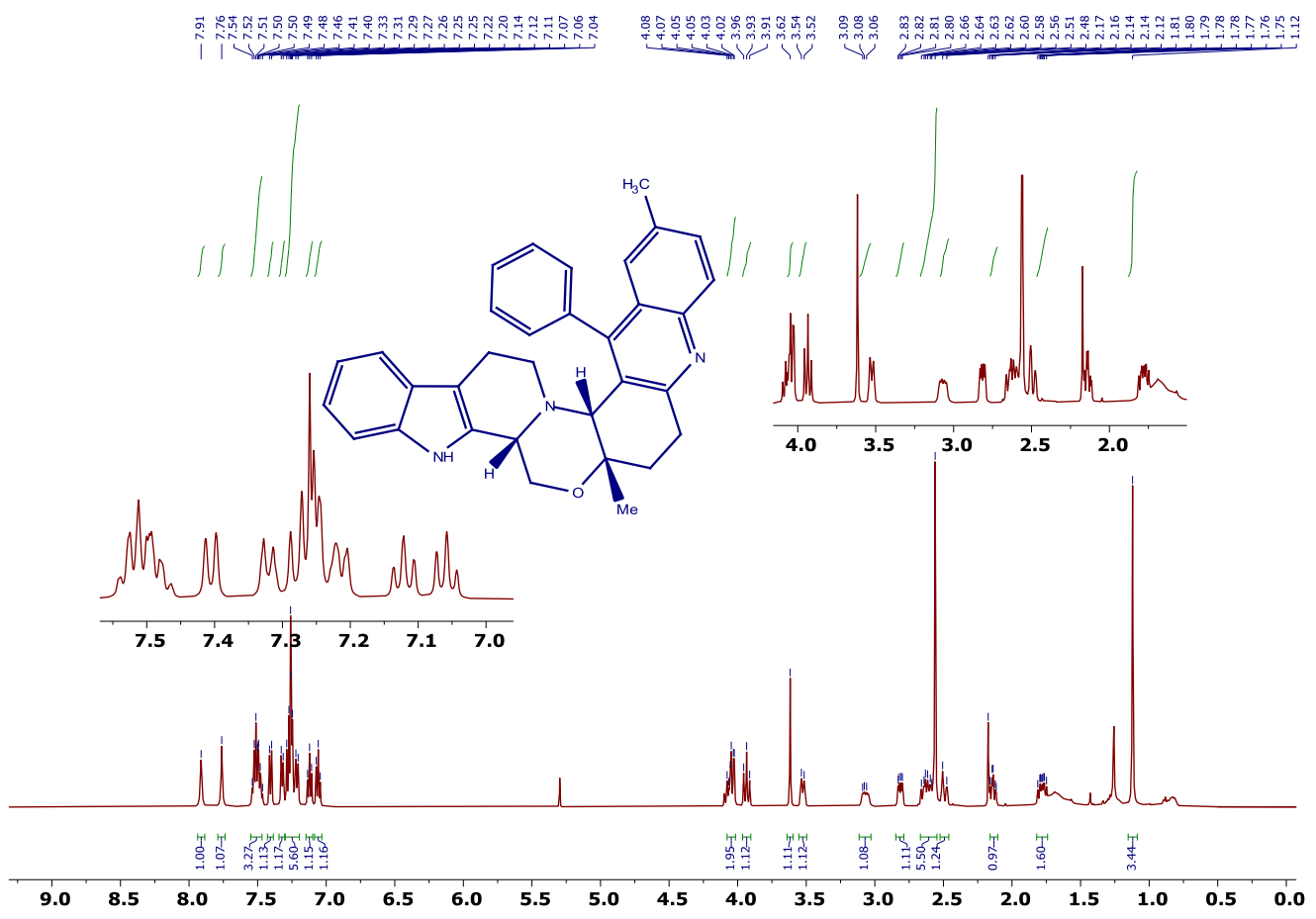

Figure S118 ${ }^{1} \mathrm{H}$ NMR (500 MHz, $\mathrm{CDCl}_{3}$ ) spectrum of (5bS,7aS,15bR)-7a,15-dimethyl5b,6,7a,8,9,15b,17,18-octahydro-5H-indolo[2",3":3',4']pyrido[1',2':4,5][1,4] oxazino[3,2a]acridine (33b)
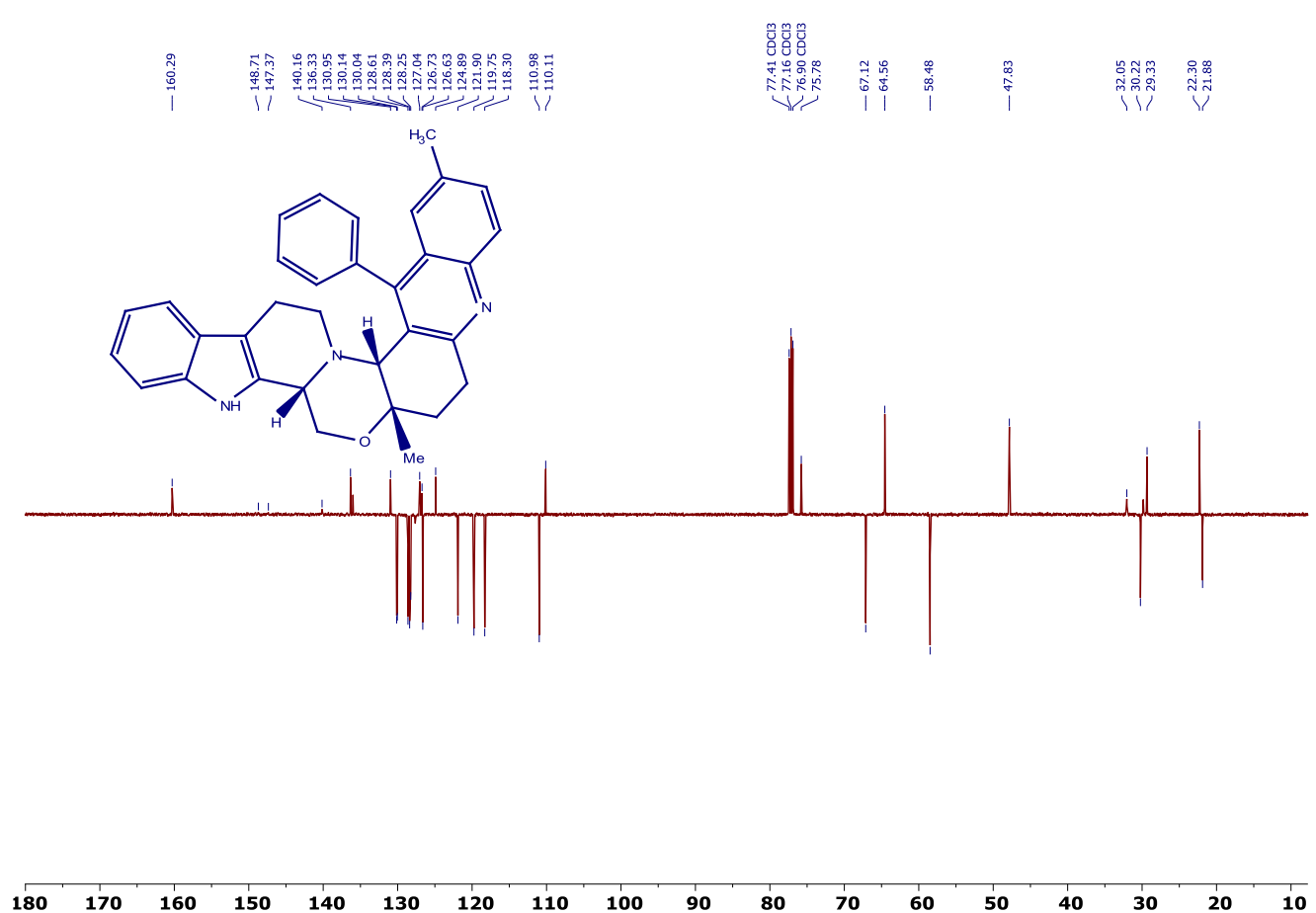

Figure S119 ${ }^{13} \mathrm{C}\left\{{ }^{1} \mathrm{H}\right\}$ APT NMR (125 MHz, $\left.\mathrm{CDCl}_{3}\right)$ spectrum of (5bS,7aS, 15bR)-7a,15dimethyl-5b,6,7a,8,9,15b,17,18-octahydro-5Hindolo[2",3":3',4']pyrido[1',2':4,5][1,4] oxazino[3,2-a]acridine (33b) 


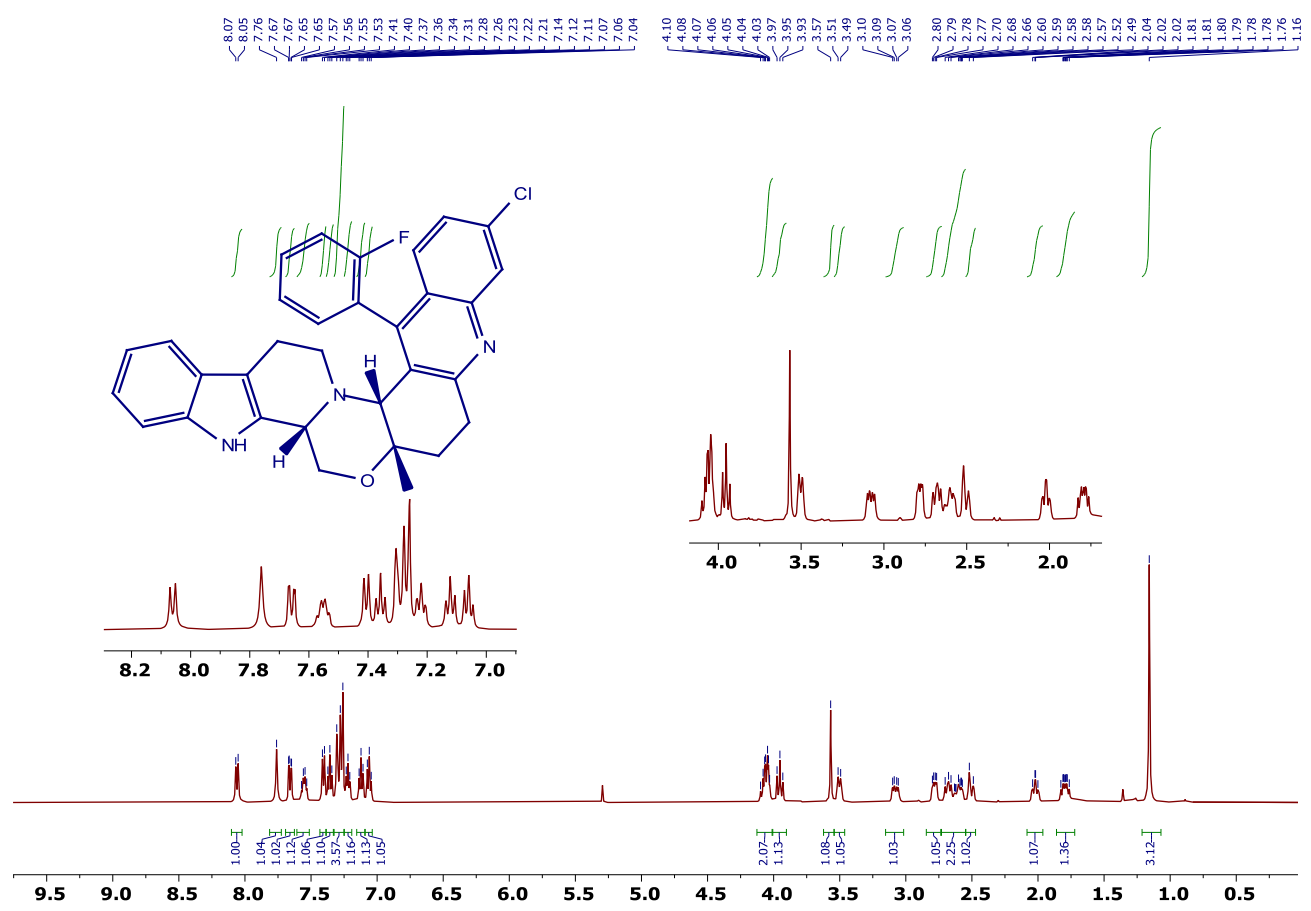

Figure S120 ${ }^{1} \mathrm{H}$ NMR (500 MHz, $\mathrm{CDCl}_{3}$ ) spectrum of (5bS,7aS, 15bR)-12-chloro-15-(2-

fluorophenyl)-7a-methyl-5b,6,7a,8,9,15b,17,18-octahydro-5Hindolo[2",3":3',4']pyrido[1',2':4,5][1,4] oxazino[3,2-a]acridine (33c)

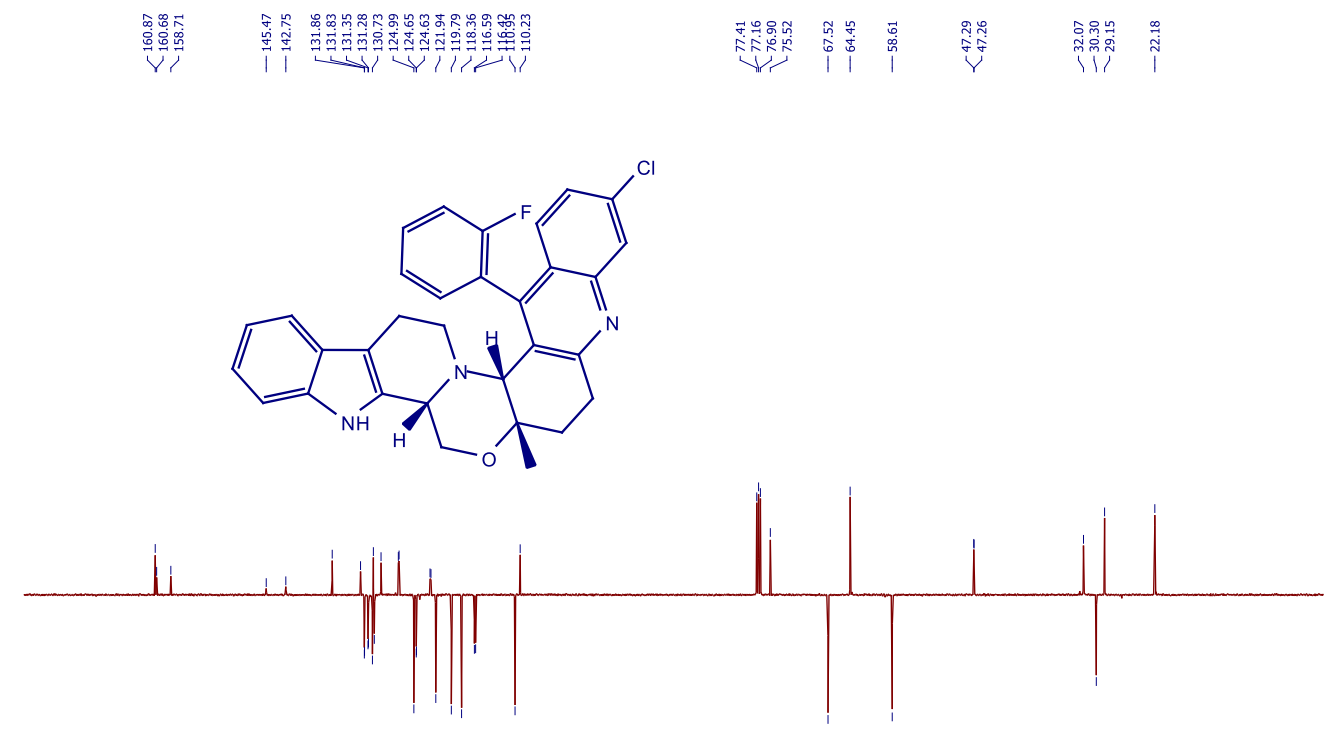

$\begin{array}{lllllllllllllllllllllll}170 & 160 & 150 & 140 & 130 & 120 & 110 & 100 & 90 & 80 & 70 & 60 & 50 & 40 & 30 & 20 & 10 & 0\end{array}$

Figure S121 ${ }^{13} \mathrm{C}\left\{{ }^{1} \mathrm{H}\right\}$ APT NMR (125 MHz, $\mathrm{CDCl}_{3}$ ) spectrum of (5bS,7aS, 15bR)-12-chloro-15(2-fluorophenyl)-7a-methyl-5b,6,7a,8,9,15b,17,18-octahydro-5Hindolo[2",3": 3',4']pyrido[1',2':4,5][1,4] oxazino[3,2-a]acridine (33c) 


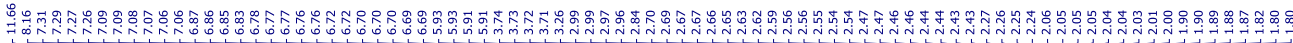

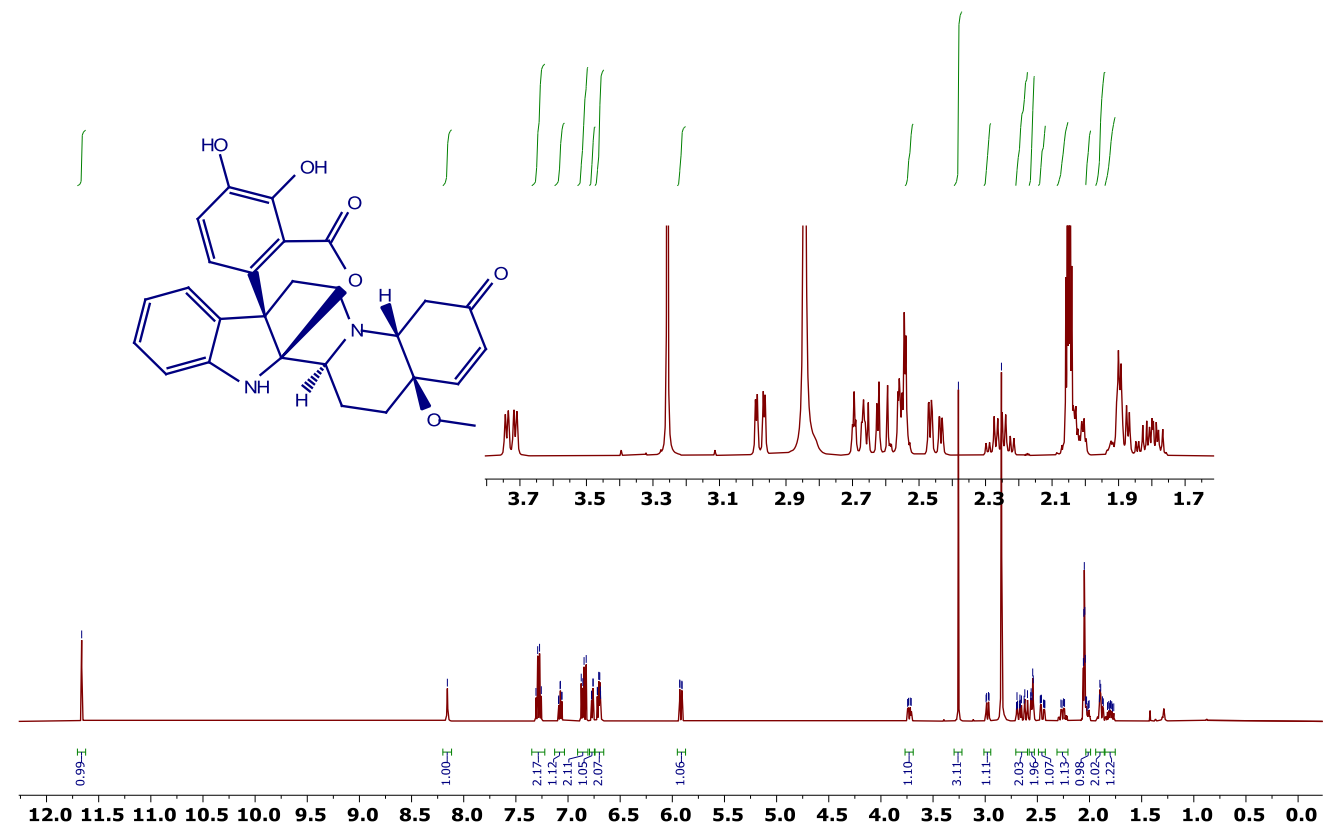

Figure S122 ${ }^{1} \mathrm{H}$ NMR (500 MHz, Acetone-d ${ }_{6}$ ) spectrum of (6aS,6bS,8aR,12aR, 15aR)-3,4-

dihydroxy-8a-methoxy-6b,7,8,8a,12,12a,14,15-octahydro-5H,11H-6a,15a(epimino[1,2]benzeno)isochromeno[3',4':3,4]pyrido[1,2-a]quinoline-5,11-dione (35a)

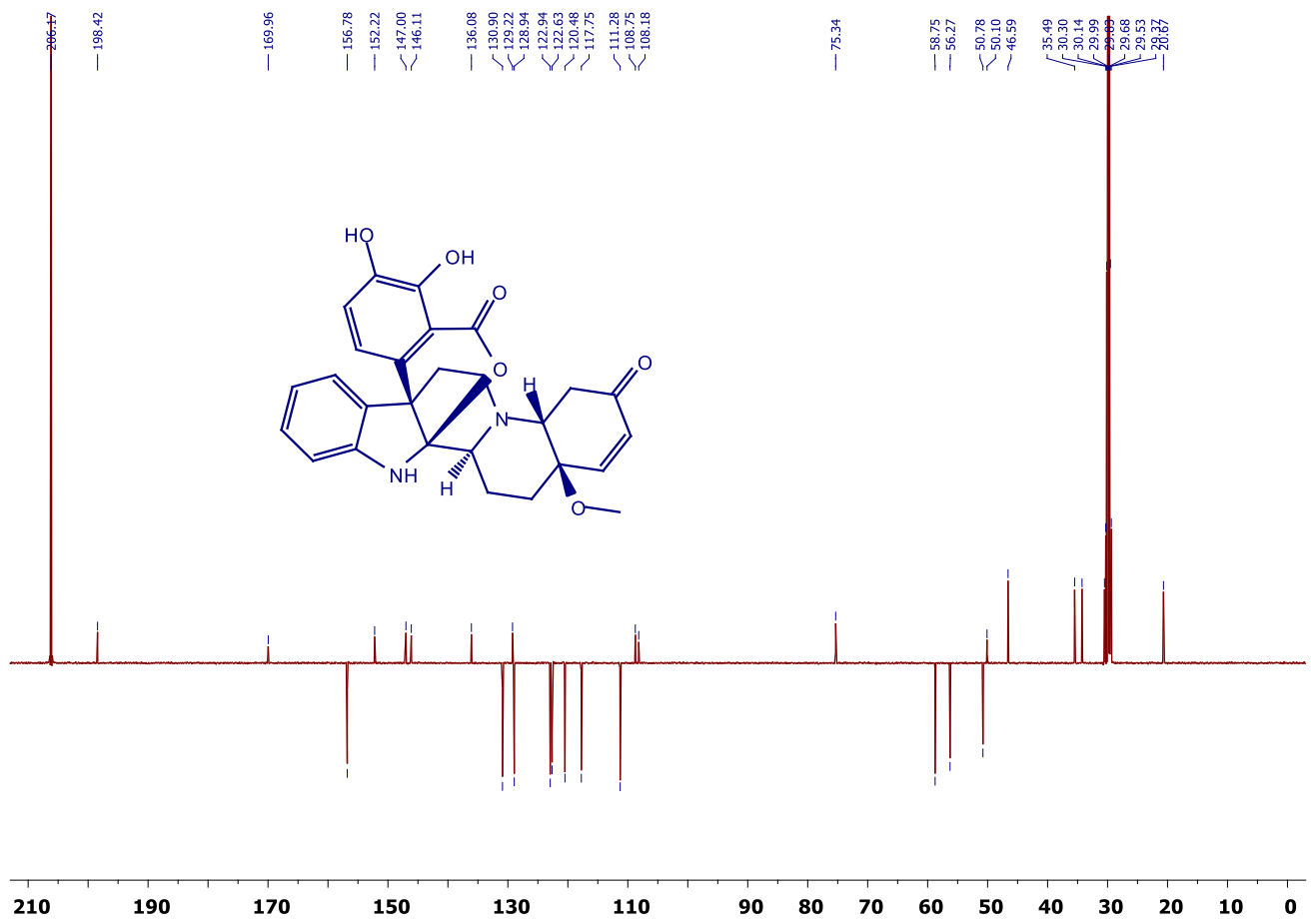

Figure S123 ${ }^{13} \mathrm{C}\left\{{ }^{1} \mathrm{H}\right\}$ APT NMR (125 MHz, Acetone-d $\left.{ }_{6}\right)$ spectrum of (6aS,6bS,8aR,12aR,15aR)3,4-dihydroxy-8a-methoxy-6b,7,8,8a,12,12a,14,15-octahydro-5H,11H-6a,15a(epimino[1,2]benzeno)isochromeno[3',4':3,4]pyrido[1,2-a]quinoline-5,11-dione (35a) 


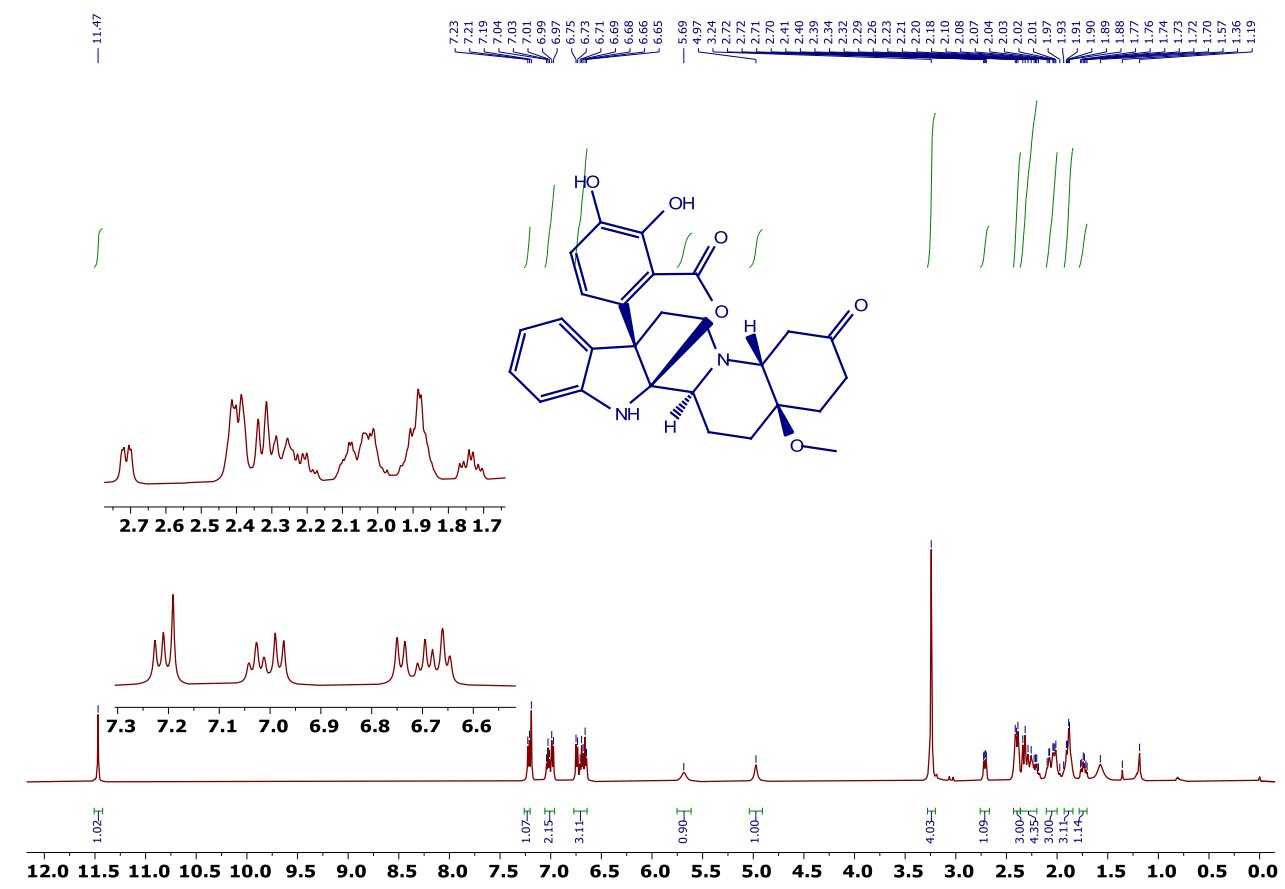

Figure S124 ${ }^{1} \mathrm{H}$ NMR $\left(500 \mathrm{MHz}, \mathrm{CDCl}_{3}\right)$ spectrum of (6aS,6bS,8aR,12aR,15aR)-3,4-dihydroxy8a-methoxy-6b,7,8,8a,9,10,12,12a,14,15-decahydro-5H,11H-6a,15a(epimino[1,2]benzeno)isochromeno[3',4':3,4]pyrido[1,2-a]quinoline-5,11-dione (35b)
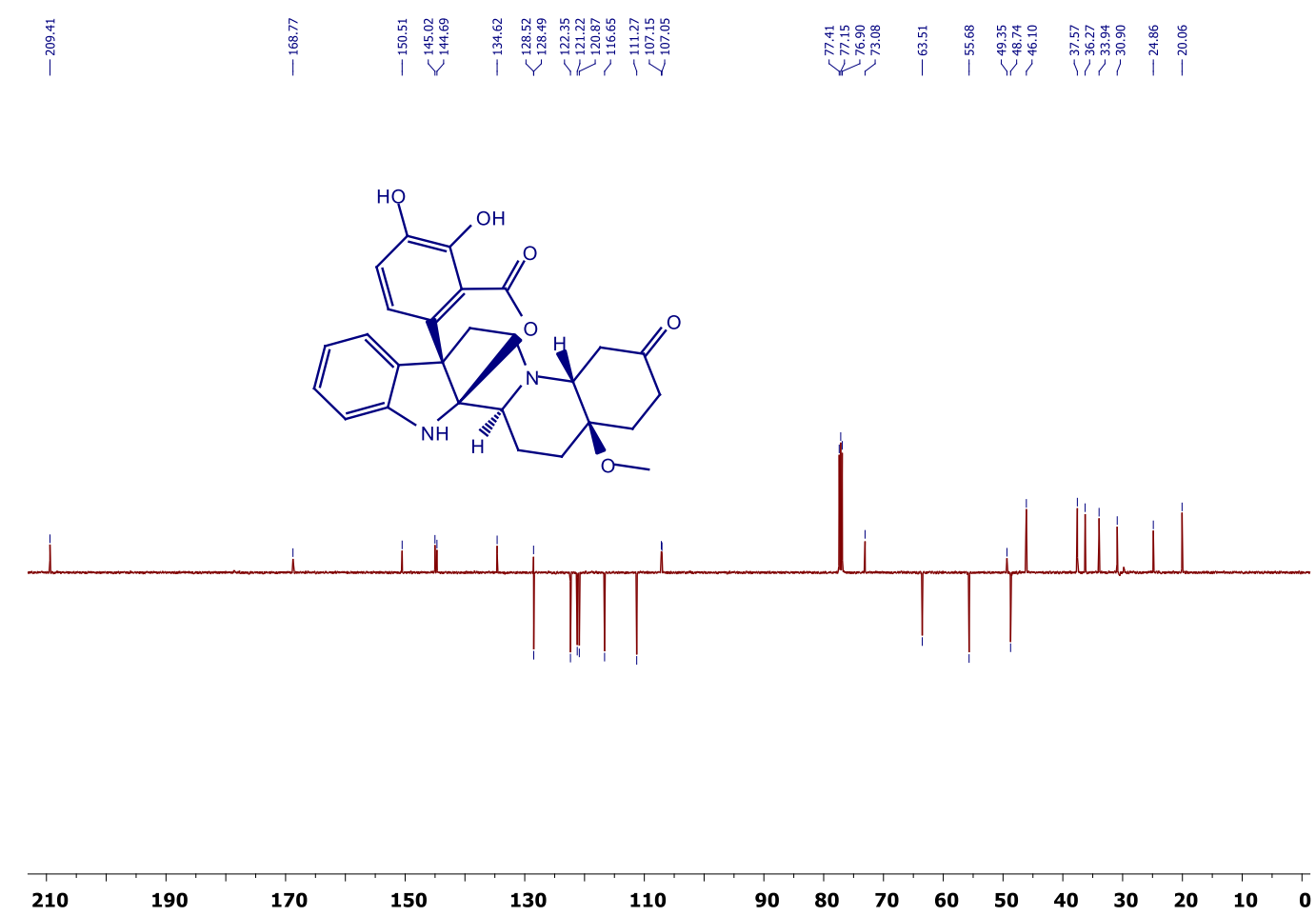

Figure S125 ${ }^{13} \mathrm{C}\left\{{ }^{1} \mathrm{H}\right\}$ APT NMR (125MHz, $\left.\mathrm{CDCl}_{3}\right)$ spectrum of (6aS,6bS,8aR,12aR,15aR)-3,4dihydroxy-8a-methoxy-6b,7,8,8a,9,10,12,12a,14,15-decahydro-5H,11H-6a,15a(epimino[1,2]benzeno)isochromeno[3',4':3,4]pyrido[1,2-a]quinoline-5,11-dione (35b) 


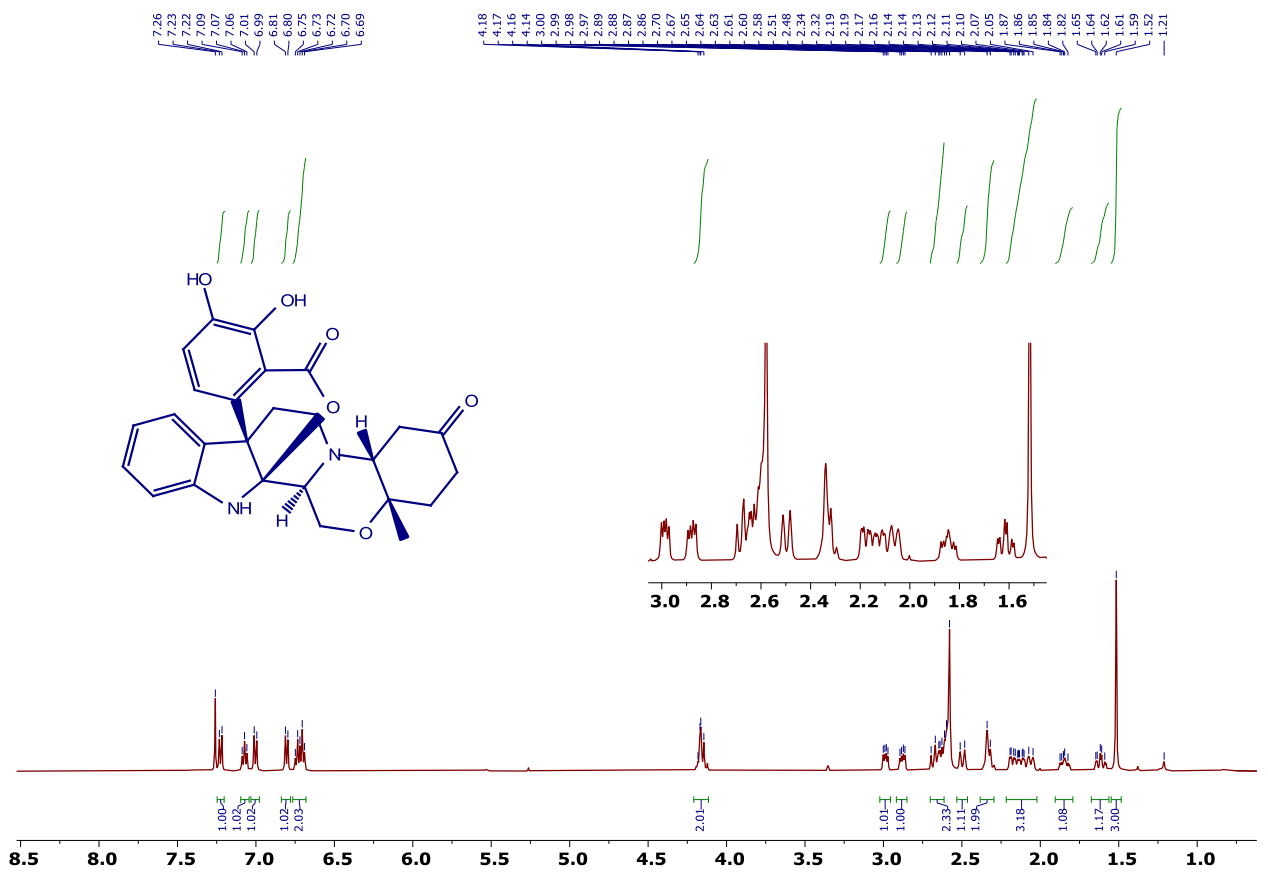

Figure S126 ${ }^{1} \mathrm{H}$ NMR $\left(500 \mathrm{MHz}, \mathrm{CDCl}_{3}\right)$ spectrum of (4aS,6aS,6bS, 12bR, 15aR)-9,10dihydroxy-4a-methyl-3,4,4a,6,6a,13,14,15a-octahydro-8H-6b,12b-

(epimino[1,2]benzeno)benzo[b]isochromeno[3',4':3,4]pyrido[1,2-d][1,4]oxazine-2,8(1H)-dione (35c)

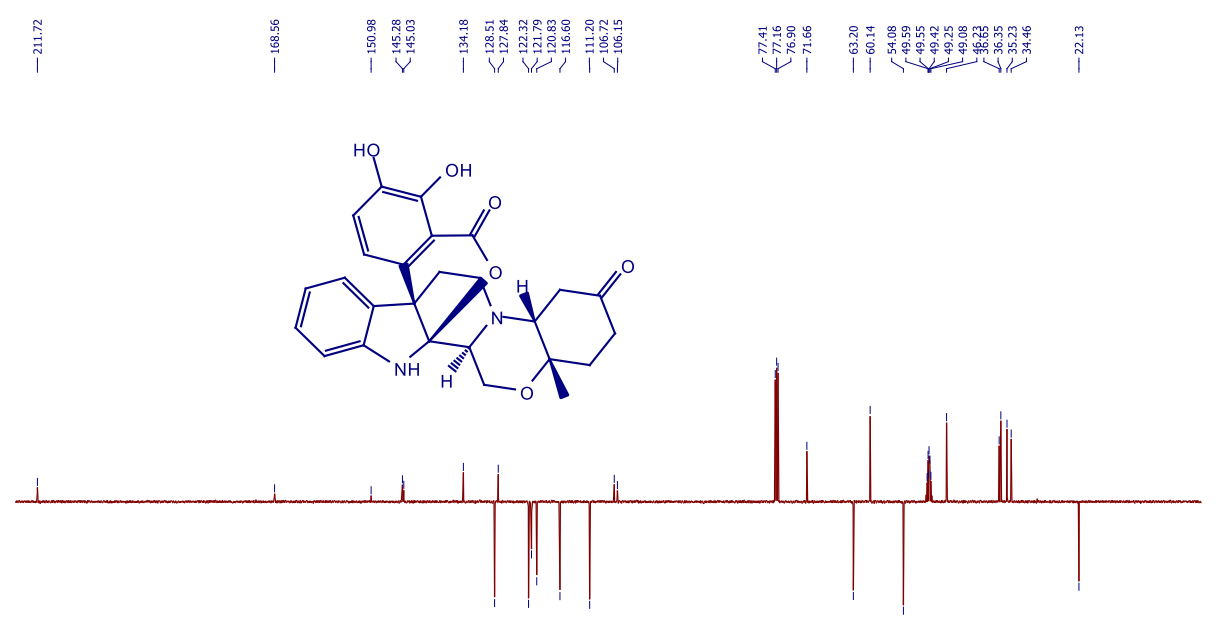

$\begin{array}{llllllllllllllllllllllll} & 210 & 190 & 170 & 150 & 130 & 110 & 90 & 80 & 70 & 60 & 50 & 40 & 30 & 20 & 10 & 0\end{array}$

Figure S127 ${ }^{13} \mathrm{C}\left\{{ }^{1} \mathrm{H}\right\}$ APT NMR (125 MHz, $\left.\mathrm{CDCl}_{3}\right)$ spectrum of $(4 \mathrm{aS}, 6 \mathrm{aS}, 6 \mathrm{bS}, 12 \mathrm{bR}, 15 \mathrm{aR})$ -

9,10-dihydroxy-4a-methyl-3,4,4a,6,6a,13,14,15a-octahydro-8H-6b,12b-

(epimino[1,2]benzeno)benzo[b]isochromeno[3',4':3,4]pyrido[1,2-d][1,4]oxazine-2,8(1H)-dione (35c) 


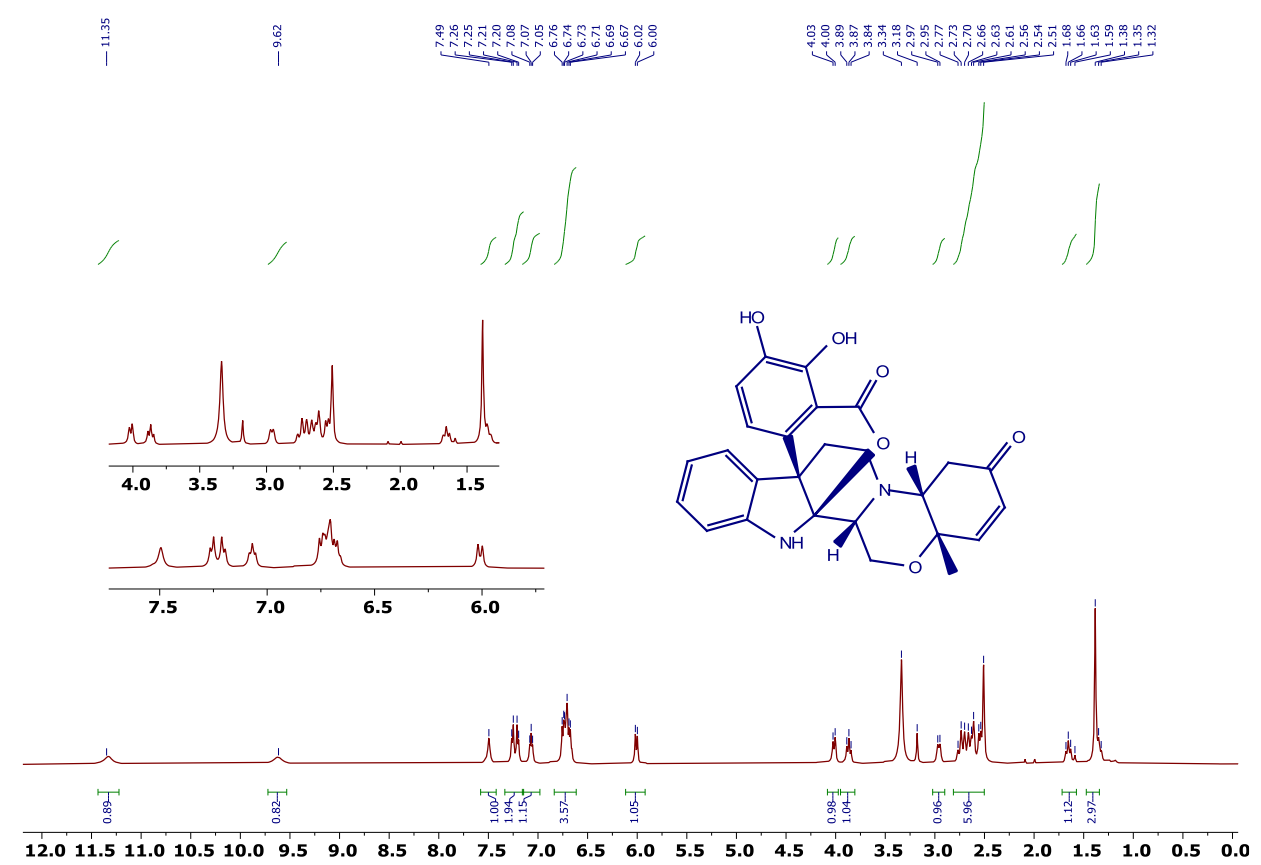

Figure S128 ${ }^{1} \mathrm{H}$ NMR (500 MHz, DMSO-d d $_{6}$ spectrum of (4aS,6aR,6bS, 12bR,15aR)-9,10dihydroxy-4a-methyl-4a,6,6a,13,14,15a-hexahydro-8H-6b,12b-

(epimino[1,2]benzeno)benzo[b]isochromeno[3',4':3,4]pyrido[1,2-d][1,4]oxazine-2,8(1H)-dione (35d)

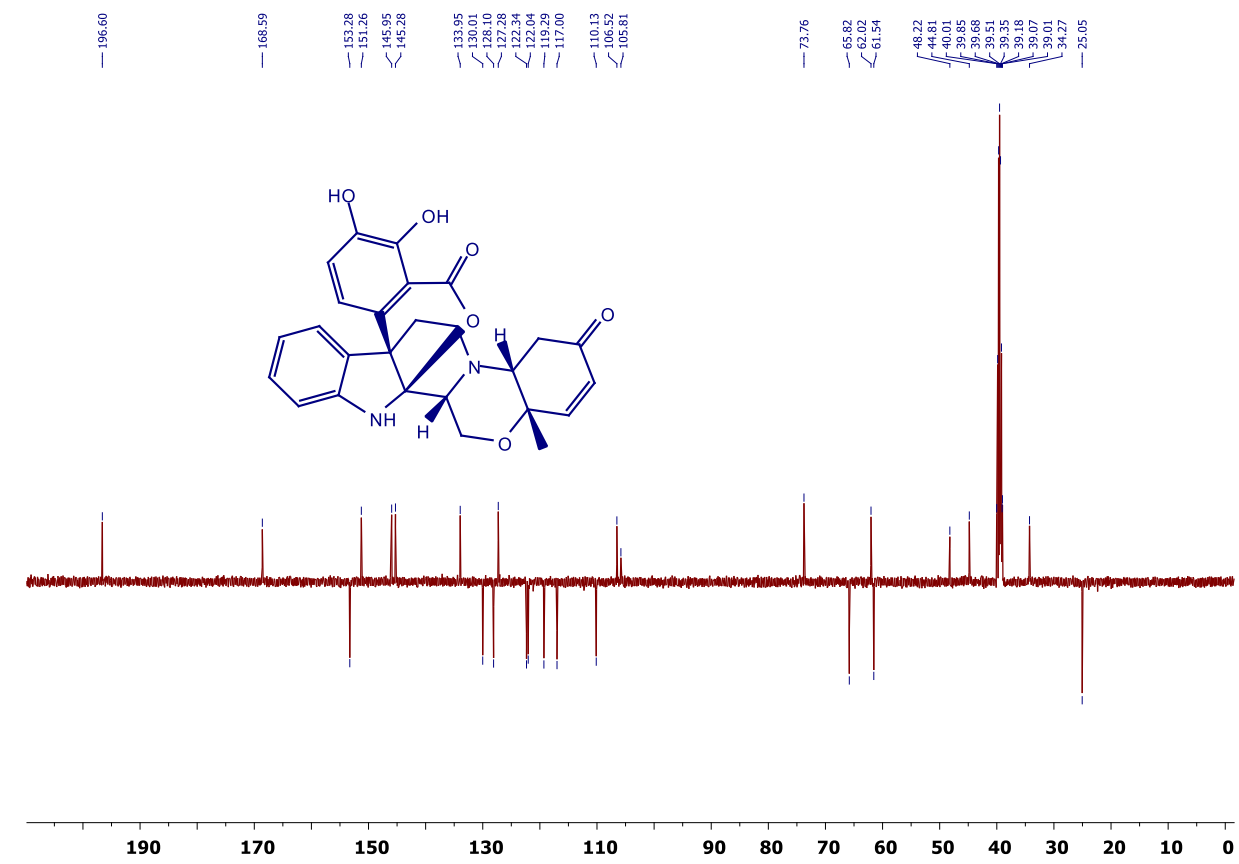

Figure S129 ${ }^{13} \mathrm{C}\left\{{ }^{1} \mathrm{H}\right\}$ APT NMR (125 MHz, DMSO-d 6 ) spectrum of (4aS,6aR,6bS,12bR,15aR)9,10-dihydroxy-4a-methyl-4a,6,6a,13,14,15a-hexahydro-8H-6b,12b-

(epimino[1,2]benzeno)benzo[b]isochromeno[3',4':3,4]pyrido[1,2-d][1,4]oxazine-2,8(1H)-dione (35d) 


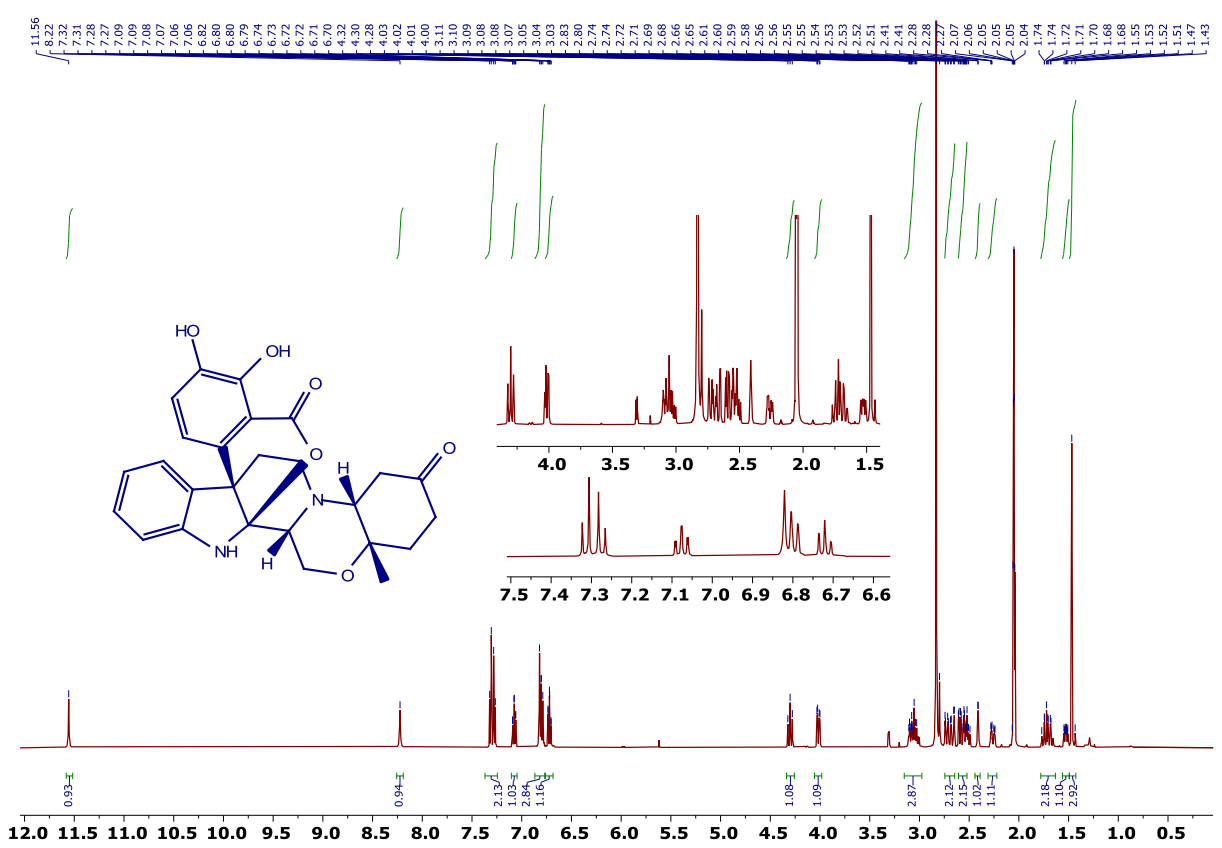

Figure S130 ${ }^{1} \mathrm{H}$ NMR (500 MHz, Acetone- $\mathrm{d}_{6}$ ) spectrum of (4aS,6aR,6bS, 12bR, 15aR)-9, 10dihydroxy-4a-methyl-3,4,4a,6,6a,13,14,15a-octahydro-8H-6b,12b(epimino[1,2]benzeno)benzo[b]isochromeno[3',4':3,4]pyrido[1,2-d][1,4]oxazine-2,8(1H)-dione (35e)

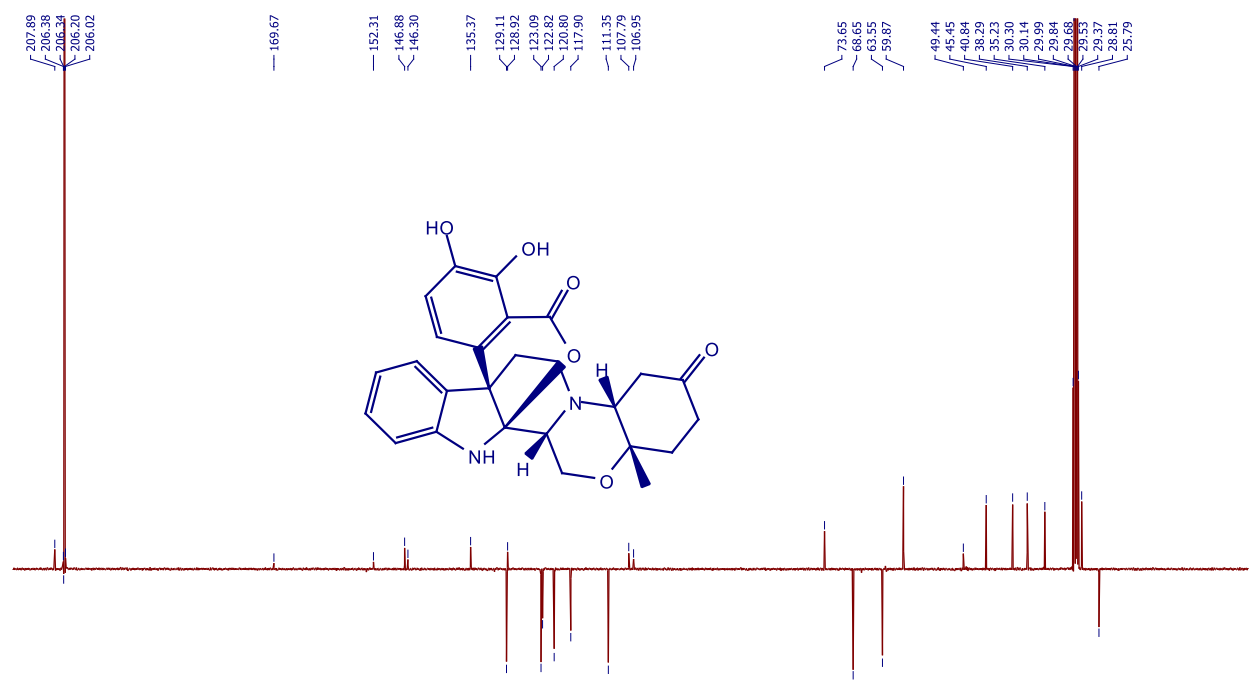

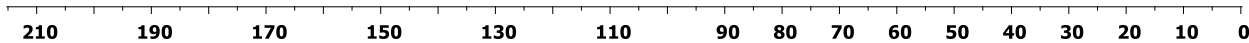

Figure S131 ${ }^{13} \mathrm{C}\left\{{ }^{1} \mathrm{H}\right\}$ APT NMR (125 MHz, Acetone- $\left.\mathrm{d}_{6}\right)$ spectrum of (4aS,6aR,6bS,12bR,15aR)9,10-dihydroxy-4a-methyl-3,4,4a,6,6a,13,14,15a-octahydro-8H-6b,12b(epimino[1,2]benzeno)benzo[b]isochromeno[3',4':3,4]pyrido[1,2-d][1,4]oxazine-2,8(1H)-dione (35e) 


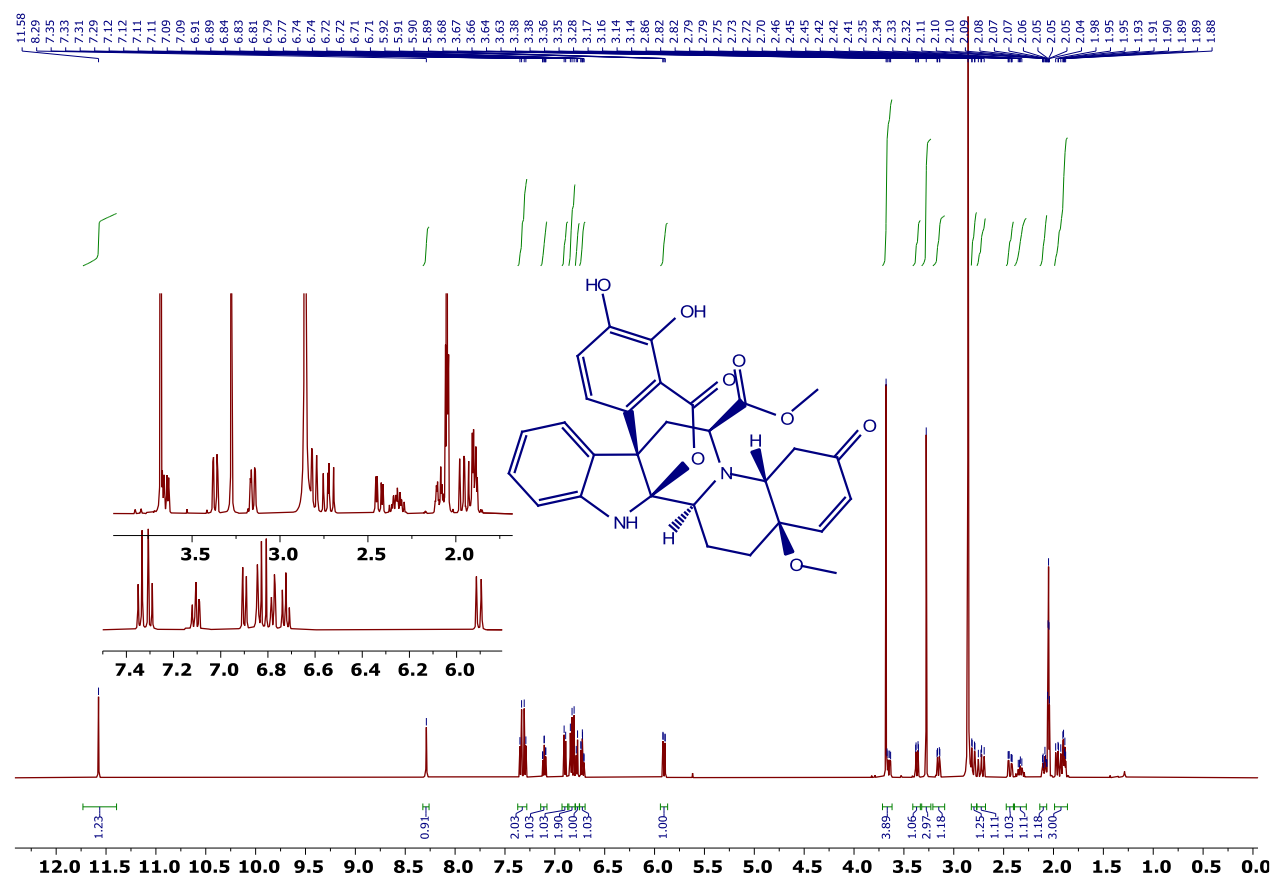

Figure S132 ${ }^{1} \mathrm{H}$ NMR (500 MHz, Acetone-d ${ }_{6}$ ) spectrum of methyl (6aS,6bS,8aR,12aR,14S,15aR)-3,4-dihydroxy-8a-methoxy-5,11-dioxo-6b,7,8a, 11,12,12a, 14,15octahydro-5H,8H-6a,15a-(epimino[1,2] benzeno)isochromeno[3',4':3,4]pyrido[1,2-a]quinoline14-carboxylate (35f)
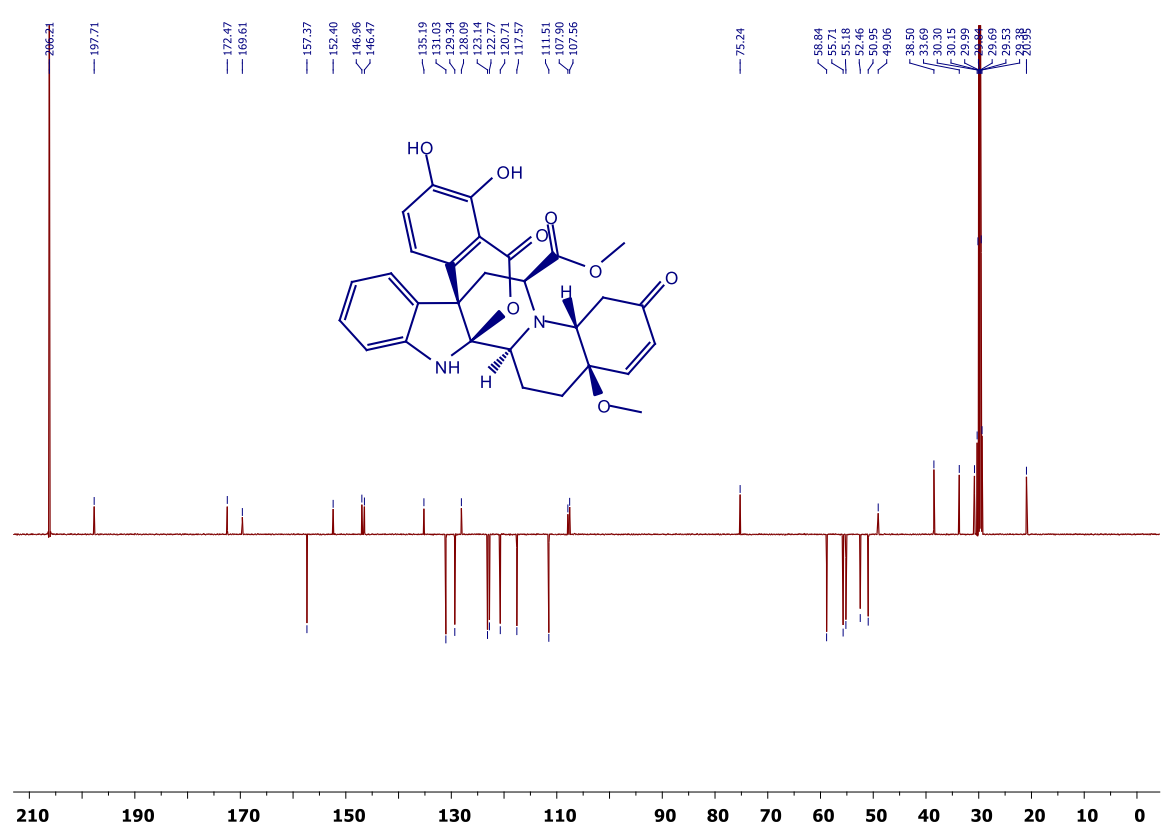

Figure S133 ${ }^{13} \mathrm{C}\left\{{ }^{1} \mathrm{H}\right\}$ APT NMR (125 MHz, Acetone-d $\left.{ }_{6}\right)$ spectrum of methyl (6aS,6bS,8aR,12aR,14S,15aR)-3,4-dihydroxy-8a-methoxy-5,11-dioxo-6b,7,8a,11,12,12a, 14,15octahydro-5H,8H-6a,15a-(epimino[1,2] benzeno)isochromeno[3',4':3,4]pyrido[1,2-a]quinoline14-carboxylate $\mathbf{( 3 5 f )}$ 


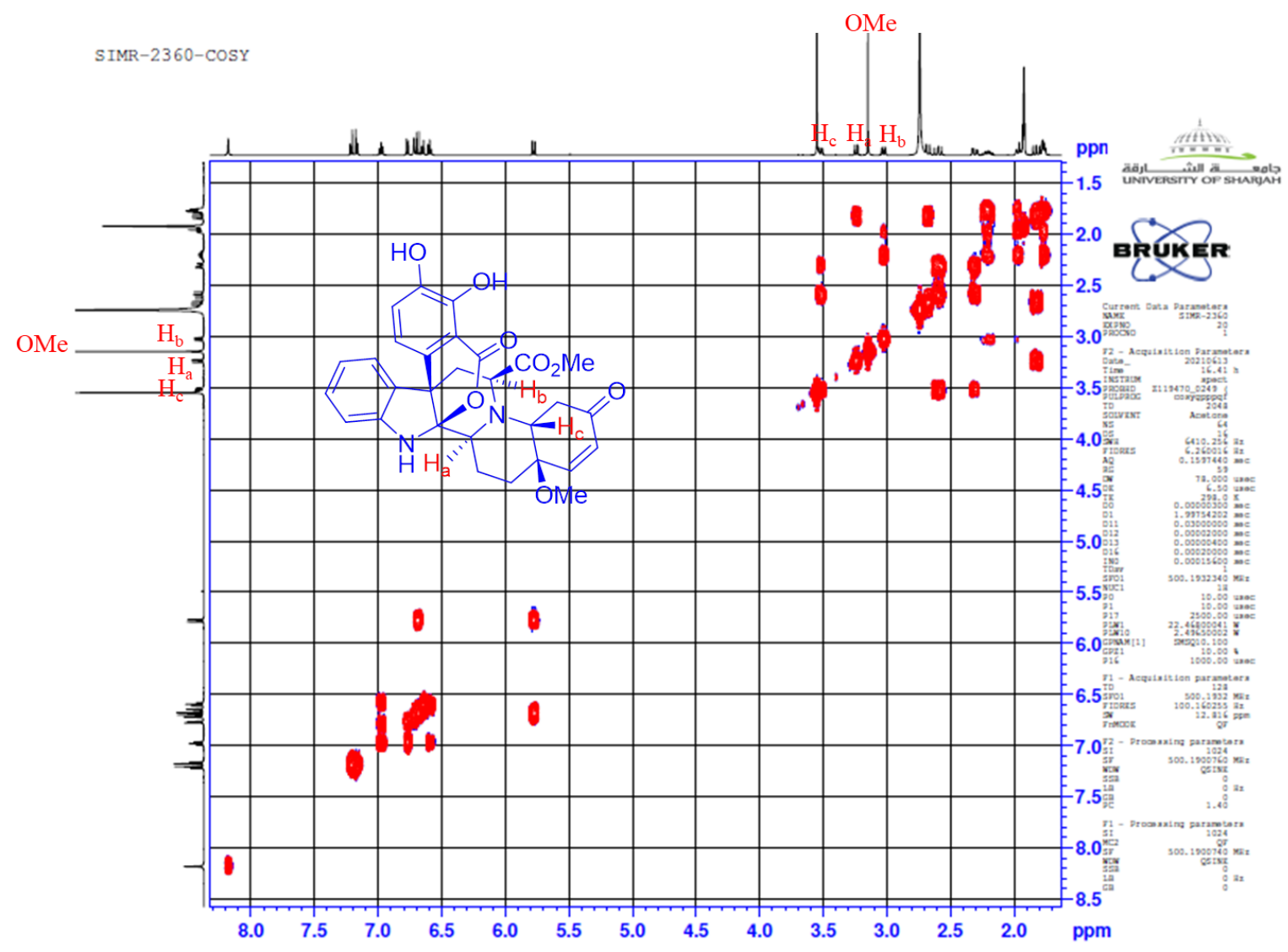

Figure S134 COSY (500 MHz, Acetone-d $)_{6}$ spectrum of methyl (6aS,6bS,8aR,12aR,14S,15aR)3,4-dihydroxy-8a-methoxy-5,11-dioxo-6b,7,8a,11,12,12a,14,15-octahydro-5H,8H-6a,15a(epimino[1,2] benzeno)isochromeno[3',4':3,4]pyrido[1,2-a]quinoline-14-carboxylate (35f) 


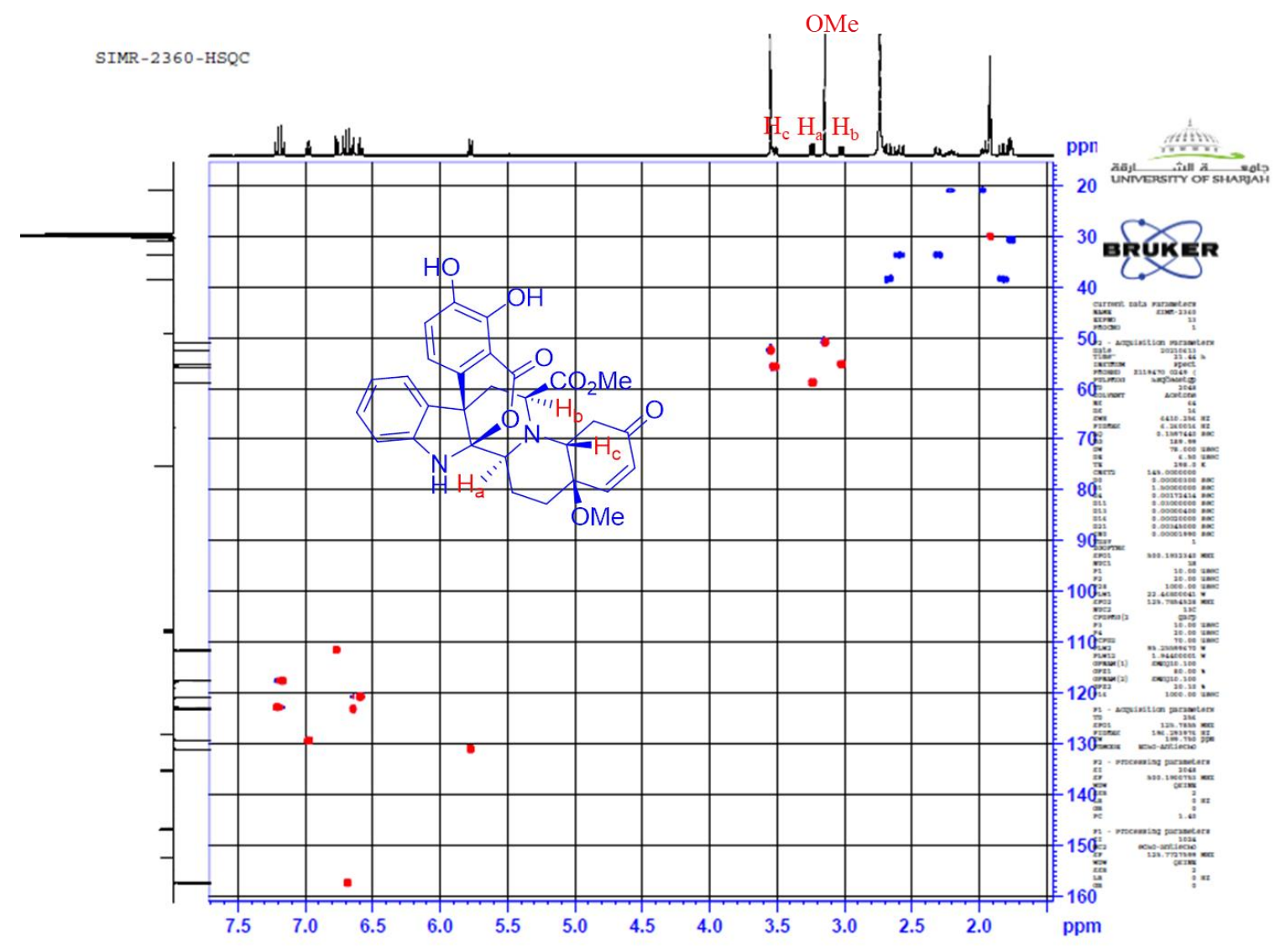

Figure S135 HSQC (500 MHz, Acetone- $\mathrm{d}_{6}$ ) spectrum of methyl (6aS,6bS,8aR, 12aR,14S,15aR)3,4-dihydroxy-8a-methoxy-5,11-dioxo-6b,7,8a,11,12,12a,14,15-octahydro-5H,8H-6a,15a(epimino[1,2] benzeno)isochromeno[3',4':3,4]pyrido[1,2-a]quinoline-14-carboxylate (35f) 


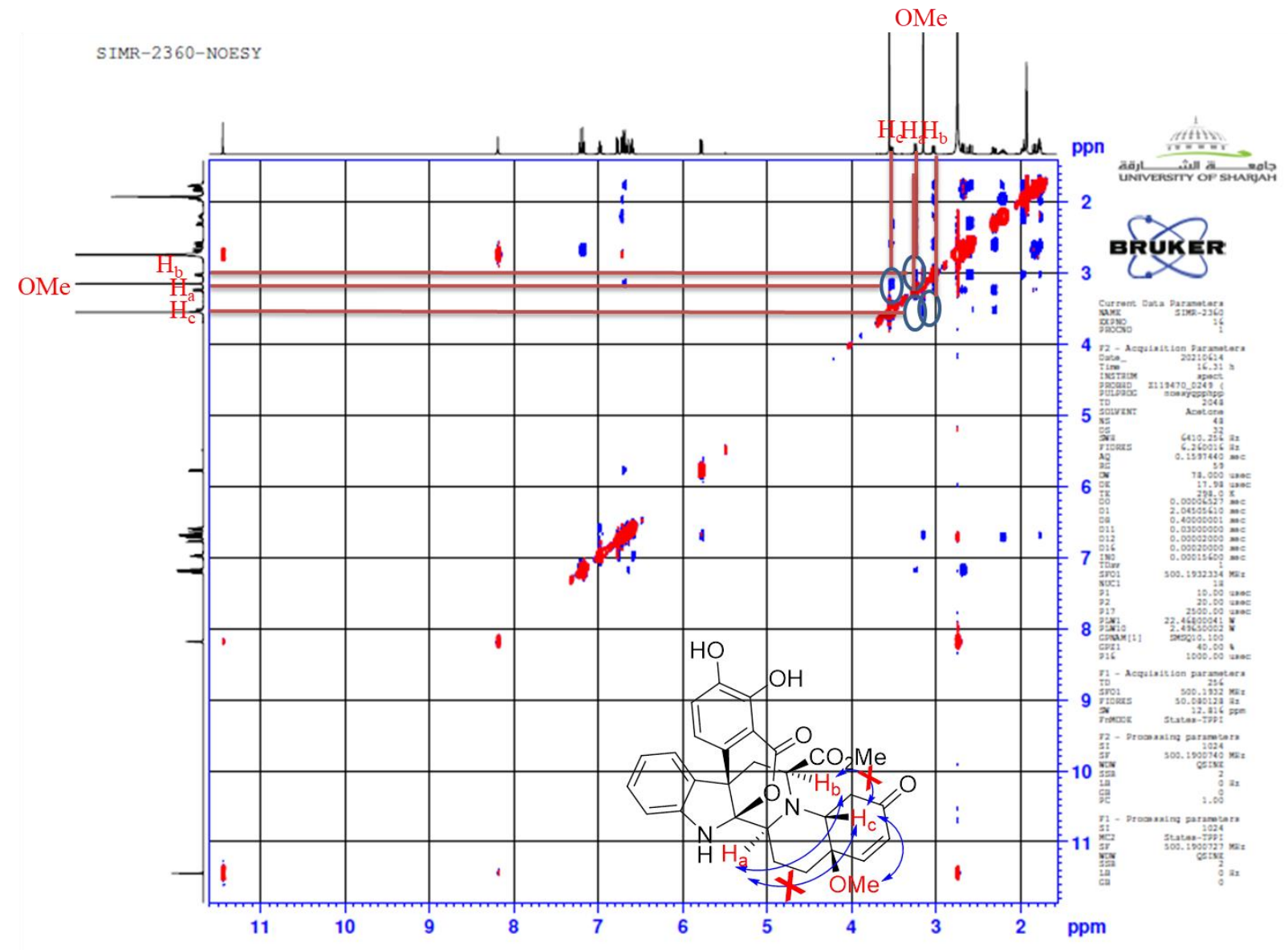

Figure S136 NOESY (500 MHz, Acetone-d $\mathrm{d}_{6}$ ) spectrum of methyl

(6aS,6bS,8aR,12aR,14S,15aR)-3,4-dihydroxy-8a-methoxy-5,11-dioxo-6b,7,8a,11,12,12a, 14,15octahydro-5H,8H-6a,15a-(epimino[1,2] benzeno)isochromeno[3',4':3,4]pyrido[1,2-a]quinoline14-carboxylate $\mathbf{( 3 5 f})$ 


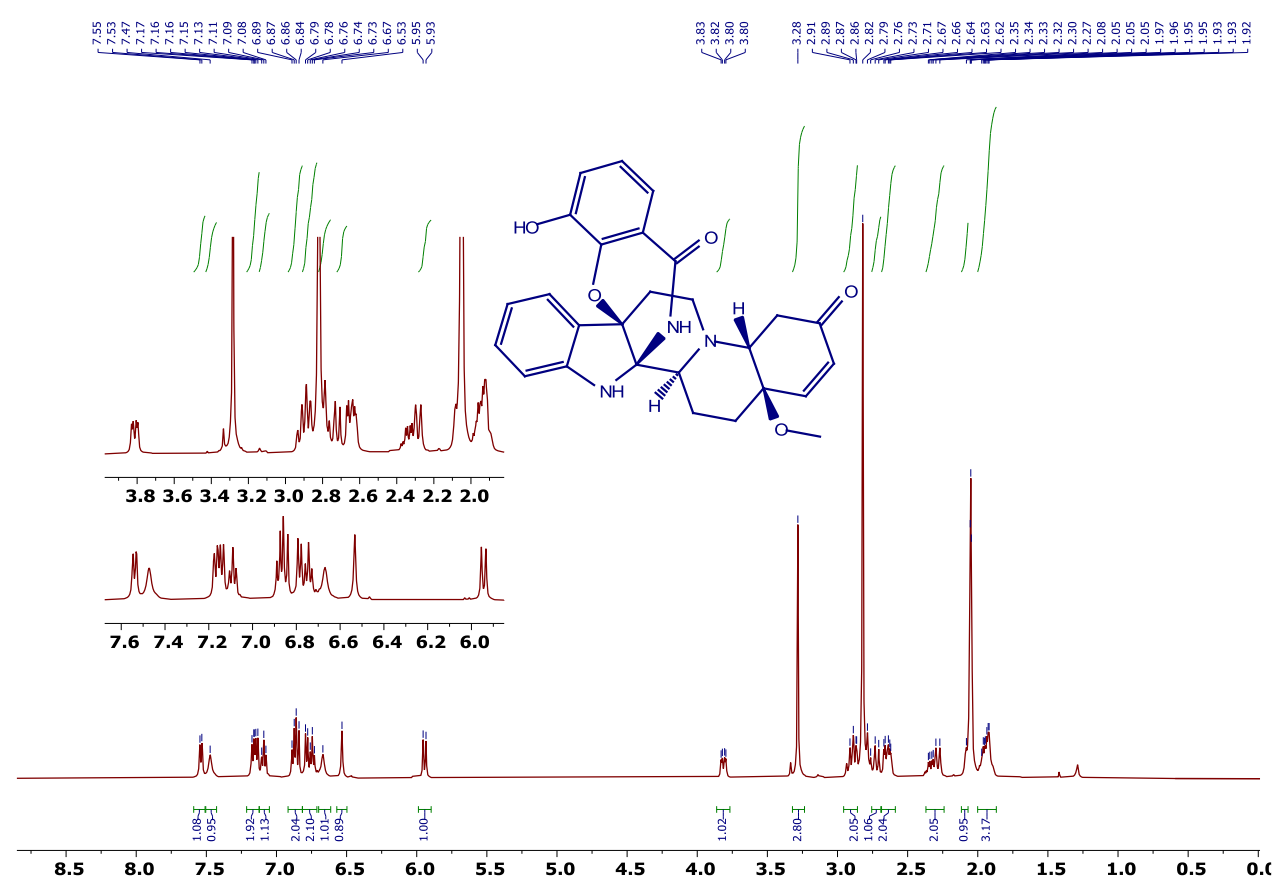

Figure S137 ${ }^{1} \mathrm{H}$ NMR $\left(500 \mathrm{MHz}\right.$, Acetone- $\left.\mathrm{d}_{6}\right)$ spectrum of (2aR,6aR,9aR,16aR,16bS)-11hydroxy-2a-methoxy-1,2,2a,6,6a,8,9,16b-octahydro-5H-16a,9a(epimino[1,2]benzeno)benzo[6',7'][1,4] oxazepino[3',2':3,4]pyrido[1,2-a]quinoline-5,15(16H)dione (37a)
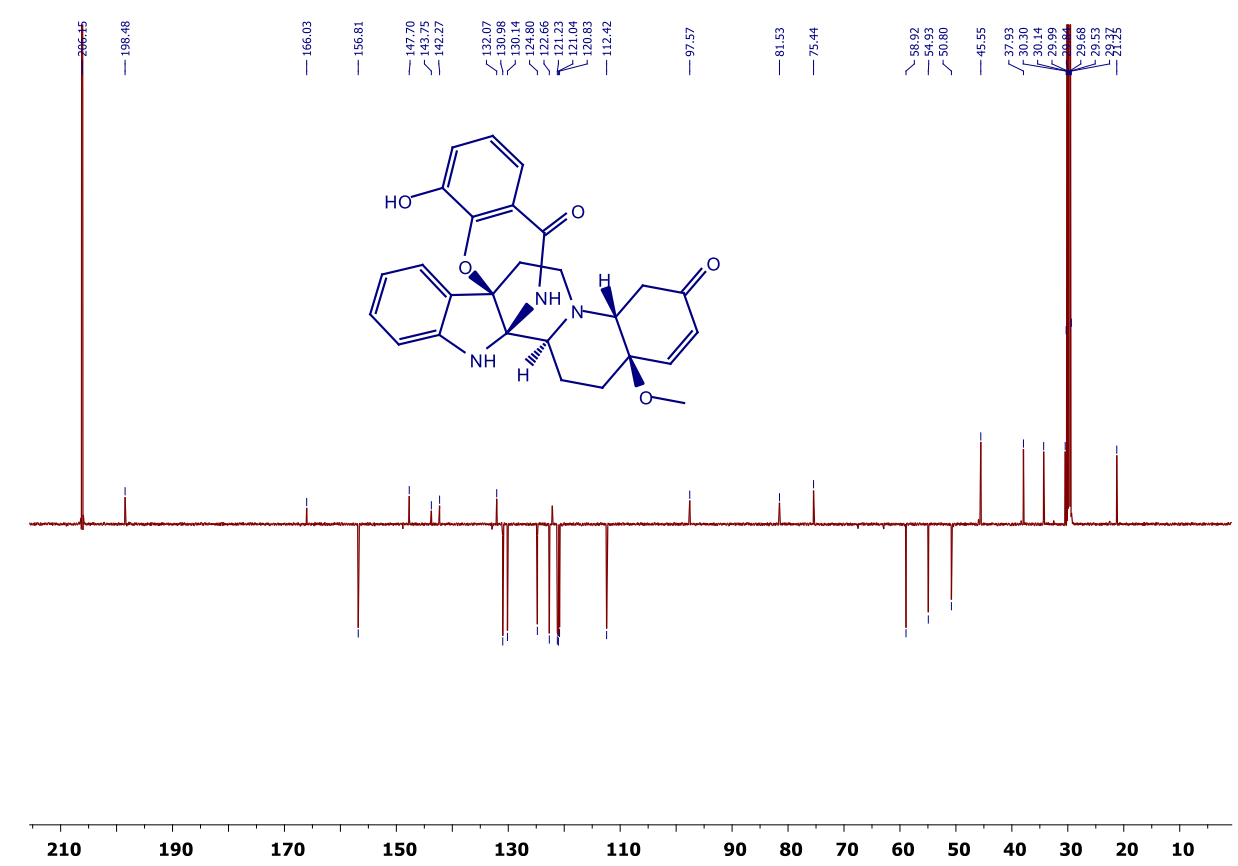

Figure S138 ${ }^{13} \mathrm{C}\left\{{ }^{1} \mathrm{H}\right\}$ APT NMR (125 MHz, Acetone-d 6 ) spectrum of (2aR,6aR,9aR,16aR,16bS)11-hydroxy-2a-methoxy-1,2,2a,6,6a,8,9,16b-octahydro-5H-16a,9a-

(epimino[1,2]benzeno)benzo[6',7'][1,4] oxazepino[3',2':3,4]pyrido[1,2-a]quinoline-5,15(16H)dione (37a) 


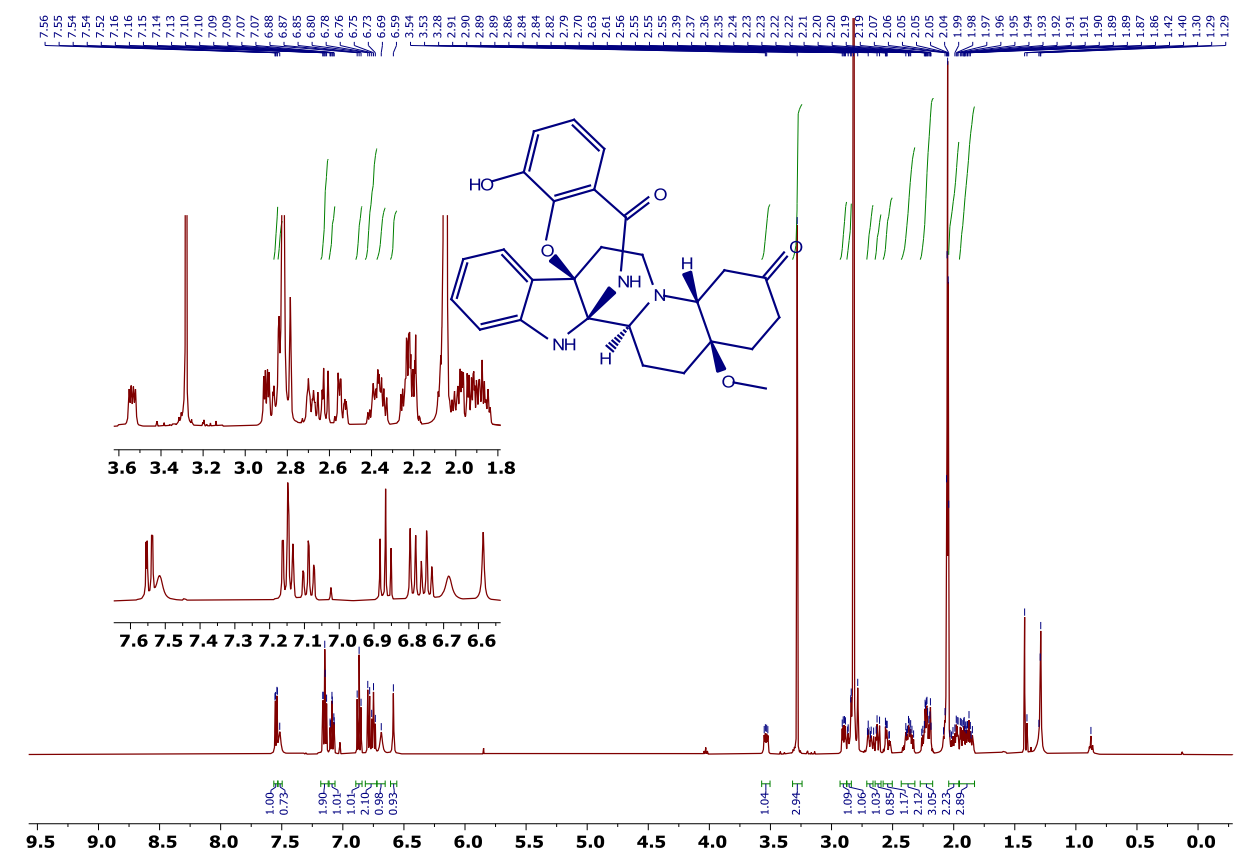

Figure S139 ${ }^{1} \mathrm{H}$ NMR $\left(500 \mathrm{MHz}\right.$, Acetone- $\left.\mathrm{d}_{6}\right)$ spectrum of (2aR,6aR,9aR,16aR,16bS)-11hydroxy-2a-methoxy-1,2,2a,3,4,6,6a, $8,9,16 \mathrm{~b}$-decahydro-5H-16a,9a(epimino[1,2]benzeno)benzo[6',7'][1,4] oxazepino[3',2':3,4]pyrido[1,2-a]quinoline-5,15(16H)dione (37b)

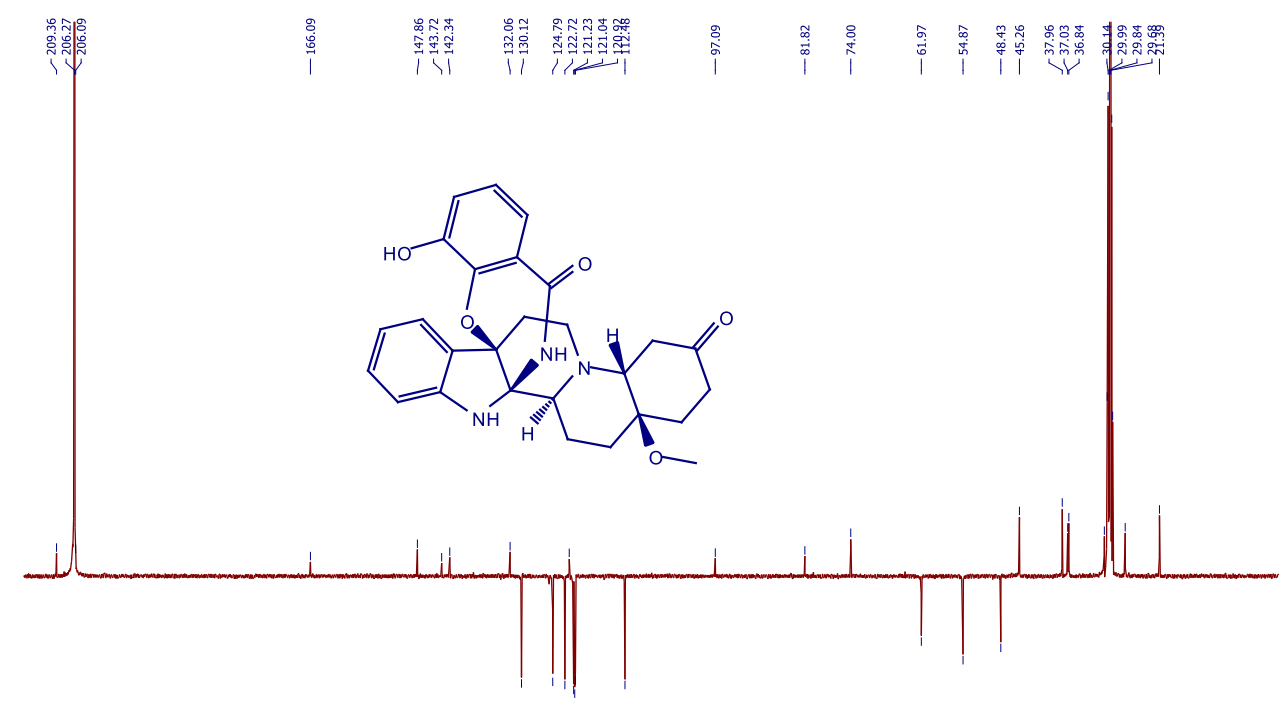

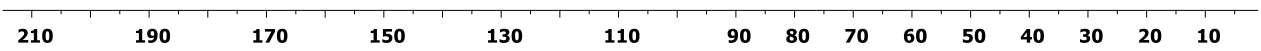

Figure S140 ${ }^{13} \mathrm{C}\left\{{ }^{1} \mathrm{H}\right\}$ APT NMR (125 MHz, Acetone-d $\left.{ }_{6}\right)$ spectrum of (2aR,6aR,9aR,16aR,16bS)-

11-hydroxy-2a-methoxy-1,2,2a,3,4,6,6a, $8,9,16 \mathrm{~b}$-decahydro-5H-16a,9a-

(epimino[1,2]benzeno)benzo[6',7'][1,4]oxazepino[3',2':3,4]pyrido[1,2-a]quinoline-5,15(16H)dione $(\mathbf{3 7 b})$ 


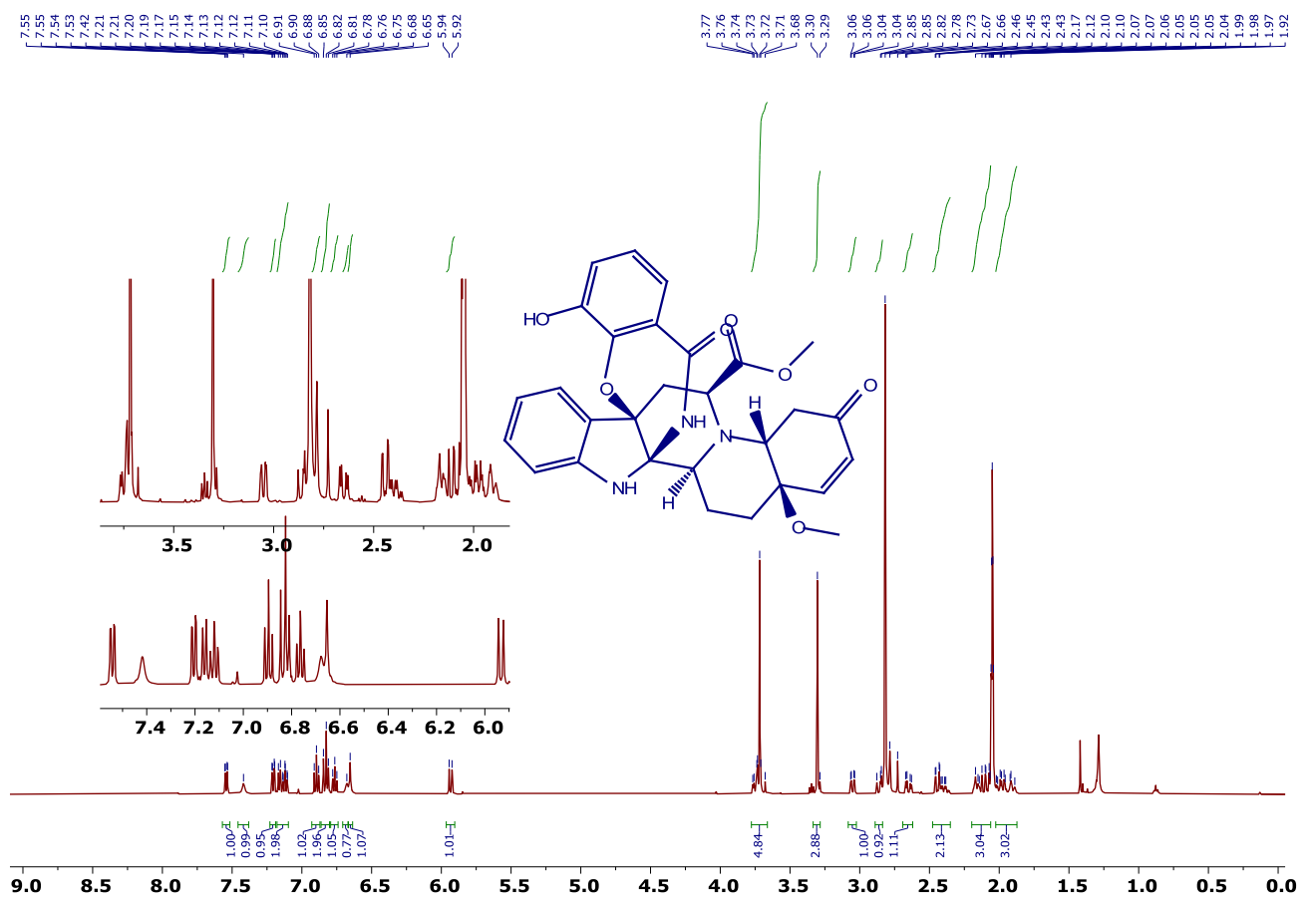

Figure S141 ${ }^{1} \mathrm{H}$ NMR $(500 \mathrm{MHz}$, Acetone-d $)$ spectrum of methyl (2aR,6aR,8S,9aR,16aR,16bS)11-hydroxy-2a-methoxy-5,15-dioxo-1,2a,5,6,6a,8,9,15,16,16b-decahydro-2H-16a,9a(epimino[1,2]benzeno)benzo[6',7'][1,4] oxazepino[3',2':3,4]pyrido[1,2-a]quinoline-8-carboxylate (37c)
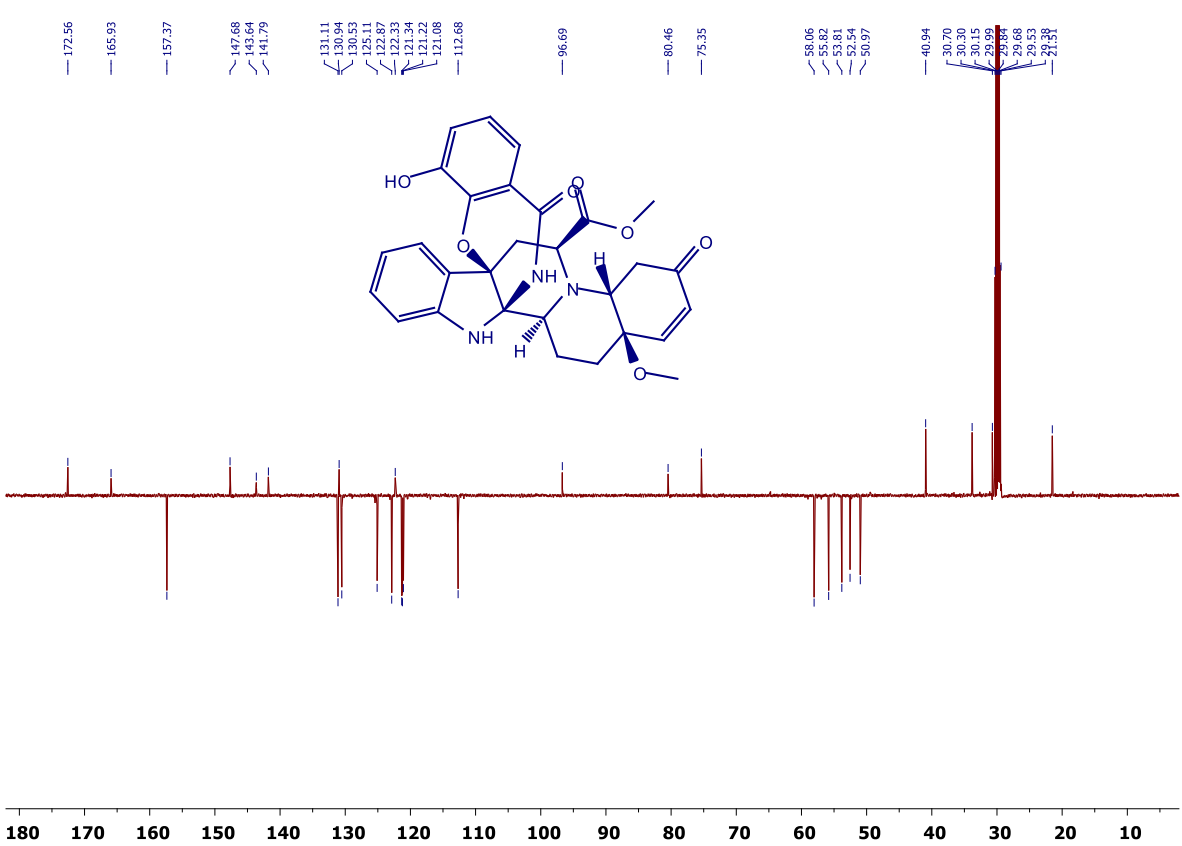

Figure S142 ${ }^{13} \mathrm{C}\left\{{ }^{1} \mathrm{H}\right\}\left(125 \mathrm{MHz}\right.$, Acetone-d $\left.\mathrm{d}_{6}\right)$ spectrum of methyl (2aR,6aR,8S,9aR,16aR,16bS)11-hydroxy-2a-methoxy-5,15-dioxo-1,2a,5,6,6a,8,9,15,16,16b-decahydro-2H-16a,9a(epimino[1,2]benzeno)benzo[6',7'][1,4] oxazepino[3',2':3,4]pyrido[1,2-a]quinoline-8-carboxylate (37c) 


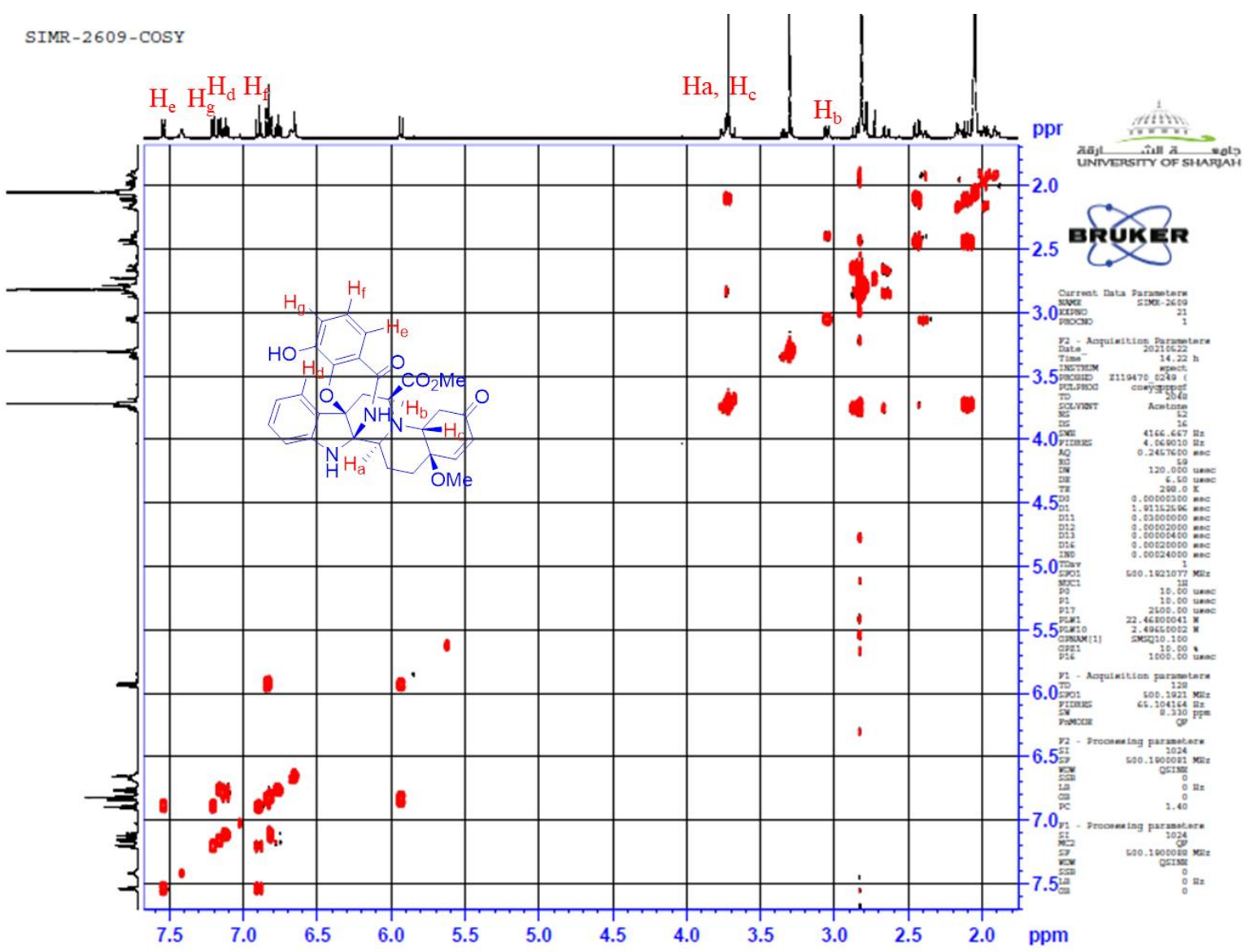

Figure S143 COSY (500 MHz, Acetone- $\mathrm{d}_{6}$ ) spectrum of methyl (2aR,6aR,8S,9aR,16aR,16bS)11-hydroxy-2a-methoxy-5,15-dioxo-1,2a,5,6,6a,8,9,15,16,16b-decahydro-2H-16a,9a(epimino[1,2]benzeno)benzo[6',7'][1,4] oxazepino[3',2':3,4]pyrido[1,2-a]quinoline-8-carboxylate (37c) 


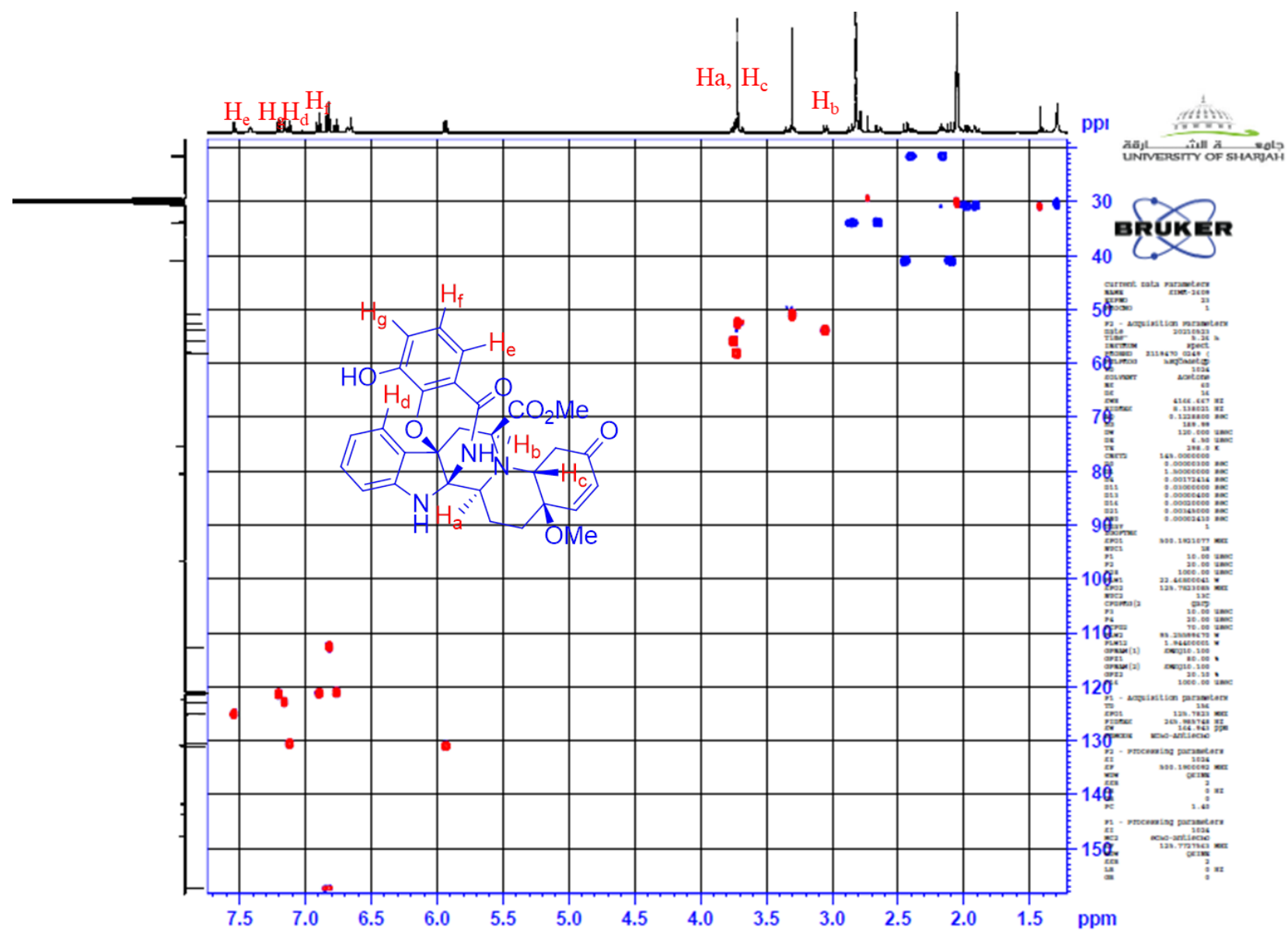

Figure S144 HSQC (500 MHz, Acetone-d $\mathrm{d}_{6}$ ) spectrum of methyl (2aR,6aR,8S,9aR,16aR,16bS)11-hydroxy-2a-methoxy-5,15-dioxo-1,2a,5,6,6a,8,9,15,16,16b-decahydro-2H-16a,9a(epimino[1,2]benzeno)benzo[6',7'][1,4] oxazepino[3',2':3,4]pyrido[1,2-a]quinoline-8-carboxylate (37c) 


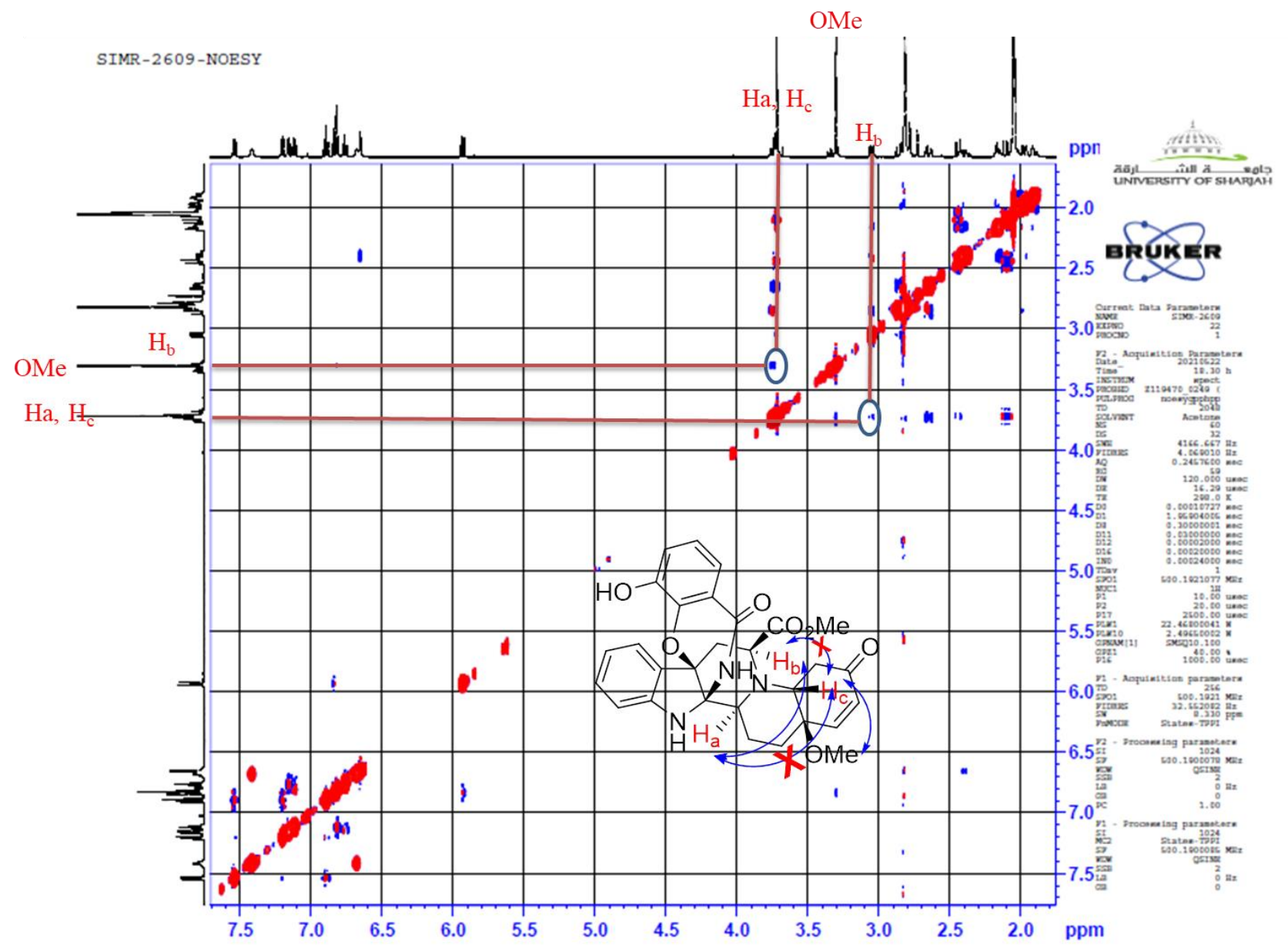

Figure S145 NOESY (500 MHz, Acetone-d $\mathrm{d}_{6}$ ) spectrum of methyl (2aR,6aR,8S,9aR,16aR,16bS)11-hydroxy-2a-methoxy-5,15-dioxo-1,2a,5,6,6a,8,9,15,16,16b-decahydro-2H-16a,9a(epimino[1,2]benzeno)benzo[6',7'][1,4] oxazepino[3',2':3,4]pyrido[1,2-a]quinoline-8-carboxylate (37c) 


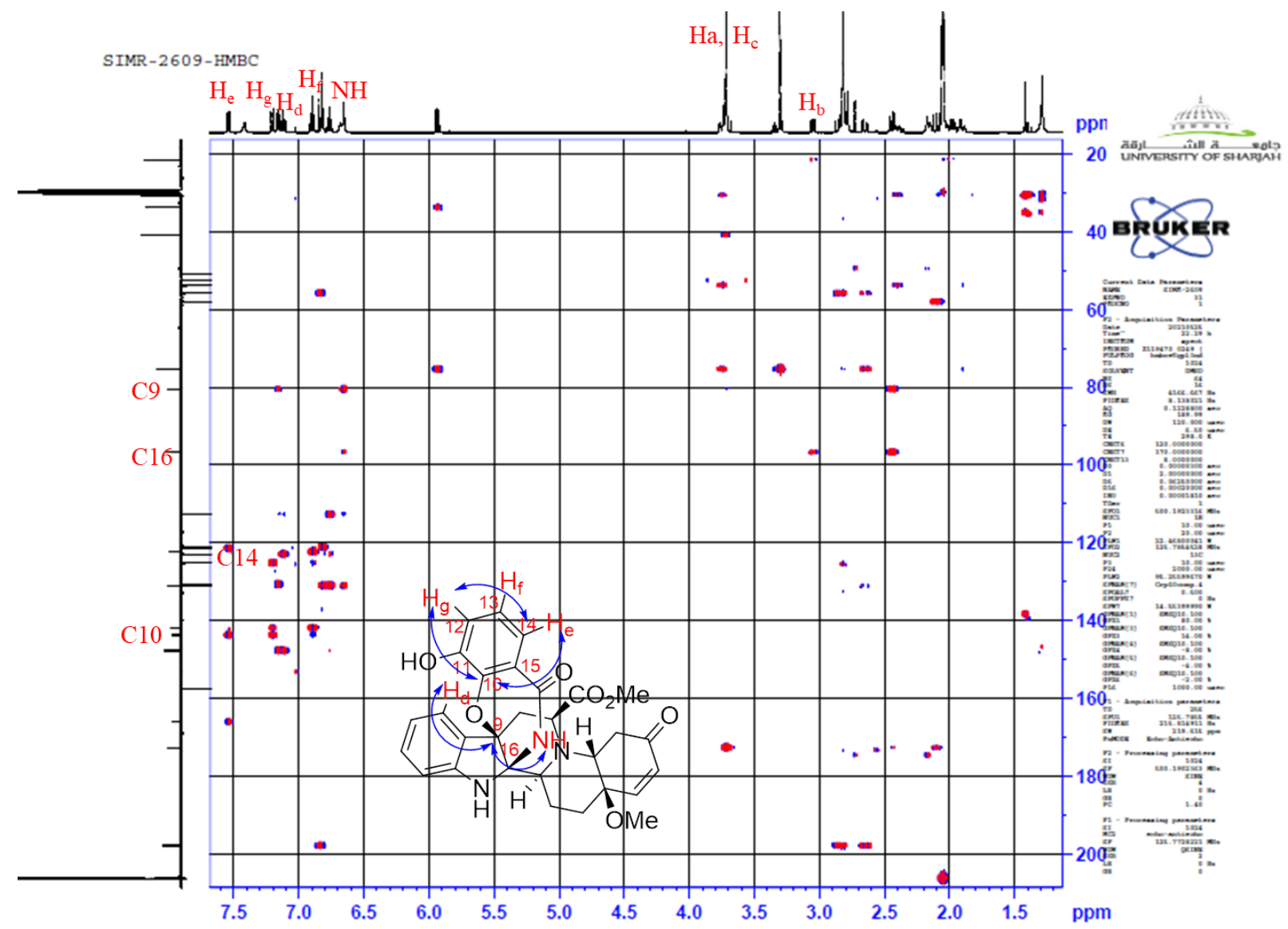

Figure S146 HMBC (500 MHz, Acetone-d $\left.\mathrm{d}_{6}\right)$ spectrum of methyl (2aR,6aR,8S,9aR,16aR,16bS)11-hydroxy-2a-methoxy-5,15-dioxo-1,2a,5,6,6a,8,9,15,16,16b-decahydro-2H-16a,9a(epimino[1,2]benzeno)benzo[6',7'][1,4] oxazepino[3',2':3,4]pyrido[1,2-a]quinoline-8-carboxylate (37c)

\section{References}

[1] V. Srinivasulu, P. Schilf, S. Ibrahim, M. A. Khanfar, S. M. Sieburth, H. Omar, A. Sebastian, R. A. AlQawasmeh, M. J. O'Connor, T. H. Al-Tel. Nat. Commun. 2018, 9, 4989. 